Lignes directrices et ouvrages de référence du CAD

\title{
La gestion de l'aide
}

PRATIQUES DES PAYS MEMBRES DU CAD

\section{OCDE $《 O$}


Lignes directrices et ouvrages de référence du CAD

\section{La gestion de l'aide : pratiques des pays membres du CAD}




\section{ORGANISATION DE COOPÉRATION ET DE DÉVELOPPEMENT ÉCONOMIQUES}

L'OCDE est un forum unique en son genre où les gouvernements de 30 démocraties œuvrent ensemble pour relever les défis économiques, sociaux et environnementaux, que pose la mondialisation. L'OCDE est aussi à l'avant-garde des efforts entrepris pour comprendre les évolutions du monde actuel et les préoccupations qu'elles font naître. Elle aide les gouvernements à faire face à des situations nouvelles en examinant des thèmes tels que le gouvernement d'entreprise, l'économie de l'information et les défis posés par le vieillissement de la population. L'Organisation offre aux gouvernements un cadre leur permettant de comparer leurs expériences en matière de politiques, de chercher des réponses à des problèmes communs, d'identifier les bonnes pratiques et de travailler à la coordination des politiques nationales et internationales.

Les pays membres de l'OCDE sont: l'Allemagne, l'Australie, l'Autriche, la Belgique, le Canada, la Corée, le Danemark, l'Espagne, les États-Unis, la Finlande, la France, la Grèce, la Hongrie, l'Irlande, l'Islande, l'Italie, le Japon, le Luxembourg, le Mexique, la Norvège, la Nouvelle-Zélande, les Pays-Bas, la Pologne, le Portugal, la République slovaque, la République tchèque, le Royaume-Uni, la Suède, la Suisse et la Turquie. La Commission des Communautés européennes participe aux travaux de l'OCDE.

Les Éditions OCDE assurent une large diffusion aux travaux de l'Organisation. Ces derniers comprennent les résultats de l'activité de collecte de statistiques, les travaux de recherche menés sur des questions économiques, sociales et environnementales, ainsi que les conventions, les principes directeurs et les modèles développés par les pays membres.

Cet ouvrage est publié sous la responsabilité du Secrétaire général de l’OCDE. Les opinions et les interprétations exprimées ne reflètent pas nécessairement les vues de l'OCDE ou des gouvernements de ses pays membres.

Publié en anglais sous le titre :

Managing Aid: Practices of DAC Member Countries 


\section{Avant-propos}

$L$

es 23 membres du Comité d'aide au développement (CAD) de l'OCDE ont consacré en 2003 près de 70 milliards USD, financés par l'impôt, à des activités en faveur du développement. Comment planifient-ils, gèrent-ils et évaluent-ils les retombées de ces dépenses?

C'est à cette question précisément que le présent ouvrage, prenant appui sur une étude des structures, des systèmes et des pratiques des pays membres du CAD, cherche à apporter des éléments de réponse. Je pense que sa publication vient à point nommé et que son intérêt tient au fait qu'il offre une profusion d'observations et de réflexions, fruits de nombreuses années de recherche.

Depuis sa création en 1960, le CAD est une tribune au service de l'échange d'idées et $d u$ recensement de pratiques exemplaires dans le domaine de la gestion de la coopération pour le développement. Tous les pays membres du CAD sont déterminés à optimiser l'efficacité de leurs activités de coopération pour le développement même si l'on observe que les différences entre les systèmes administratifs et législatifs et les cadres régissant la reddition de comptes se répercutent au niveau des stratégies de mise en œuure des programmes d'aide. Le présent ouvrage vise à donner aux gestionnaires de l'aide, aux spécialistes, aux étudiants et à tout autre public intéressé une idée de la diversité des solutions élaborées aux fins d'accroître les apports globaux des grands pays donneurs tout en améliorant l'échelonnement dans le temps et l'efficacité des efforts qu'ils déploient dans le domaine de la coopération pour le développement.

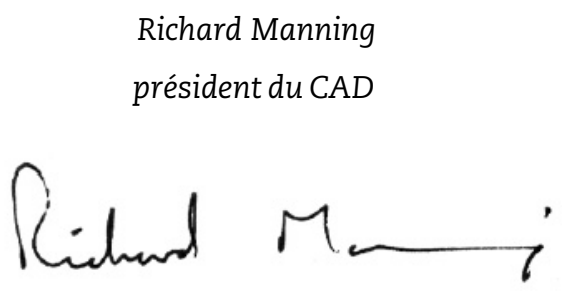


Remerciements. Le présent document de référence a vu le jour en réponse à des demandes exprimées par les pays membres du CAD, ainsi que par d'autres acteurs et observateurs du système mondial d'aide au développement, et fait suite à l'étude publiée en 1999 sous le titre "Comparaison des systèmes de gestion de la coopération pour le développement dans les pays de l'OCDE membres du CAD ». Il est le fruit des travaux que son auteur, Michael Laird, a pu mener à bien avec le concours de Jean Lennock et Julia Schweizer, assistés de Michelle Weston et Carola Miras, et a bénéficié des contributions et commentaires transmis par de nombreux collaborateurs de la Direction de la coopération pour le développement de l'OCDE pendant la phase de rédaction. Cet ouvrage a pu être réalisé grâce à des dons de l'Irlande, du Canada, du Japon, de l'Autriche et de l'Allemagne.

Pour permettre à l'OCDE de réaliser ses objectifs, un certain nombre de comités spécialisés ont été créés. L'un de ceux-ci est le Comité d'aide au développement, dont les membres ont décidé, en commun, de parvenir à un accroissement du volume total des ressources mises à la disposition des pays en développement et d'en améliorer l'efficacité. À cette fin les membres examinent, ensemble et périodiquement, à la fois le volume et la nature de leurs contributions aux programmes d'aide, établis à titre bilatéral et multilatéral, et se consultent sur toutes les autres questions importantes de leur politique d'aide.

Les membres du Comité d'aide au développement sont: l'Allemagne, l'Australie, l'Autriche, la Belgique, le Canada, le Danemark, l'Espagne, les États-Unis, la Finlande, la France, la Grèce, l'Irlande, l'Italie, le Japon, le Luxembourg, la Norvège, la Nouvelle-Zélande, les Pays-Bas, le Portugal, le Royaume-Uni, la Suède, la Suisse, et la Commission des Communautés européennes. 


\section{Table des matières}

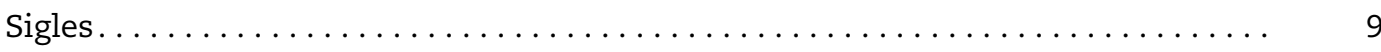

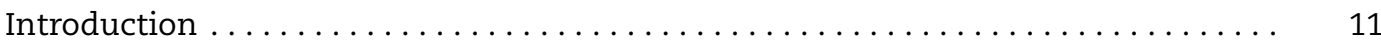

Chapitre 1. Coopération pour le développement et nouveau millénaire :

contexte, problèmes et solutions $\ldots \ldots \ldots \ldots \ldots \ldots \ldots \ldots \ldots \ldots$

La nécessité de faire face résolument aux problèmes de développement

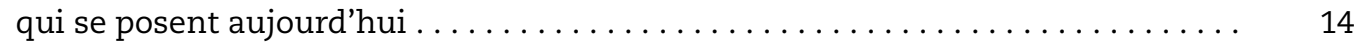

Tracer la voie à suivre. . . . . . . . . . . . . . . . . . . . . . . . . . . 15

La cohérence des politiques au service du développement .............. 20

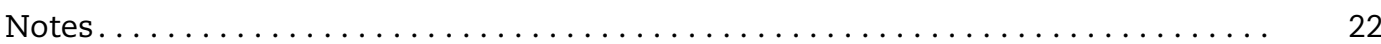

Chapitre 2. Les fondements juridiques et politiques de la coopération

pour le développement.......................... 25

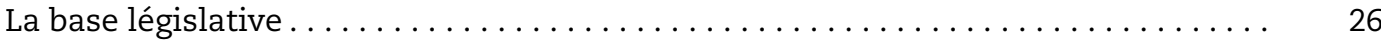

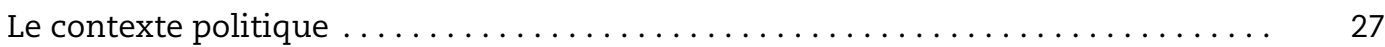

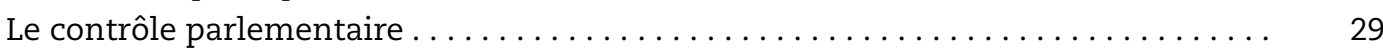

Le soutien de l'opinion publique à la coopération pour le développement....... 30

Notes............................................. 33

Chapitre 3. La politique de coopération pour le développement des pays membres

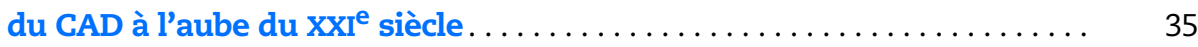

Aide aux pays en développement et en transition $\ldots \ldots \ldots \ldots \ldots \ldots \ldots \ldots \ldots \ldots$

Mécanismes de coordination entre les donneurs $\ldots \ldots \ldots \ldots \ldots \ldots \ldots \ldots . \ldots 40$

Notes................................................ 44

Chapitre 4. Origine et affectation des fonds................... 45

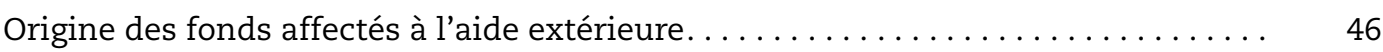

Notes............................................. 56

Chapitre 5. Profil général des systèmes de coopération pour le développement des pays membres du CAD . . . . . . . . . . . . . . . . 57

Profil des systèmes de coopération pour le développement $\ldots \ldots \ldots \ldots \ldots \ldots \ldots \quad 58$

Caractéristiques des systèmes de coopération pour le développement des pays

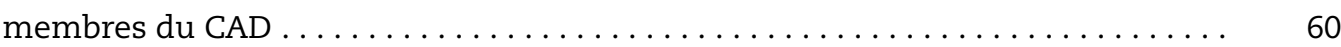

Aide extérieure fournie par des instances infranationales. . . . . . . . . . . 68 
Chapitre 6. Gestion des organismes d'aide : structures et systèmes internes ... 71

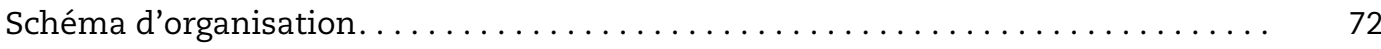

Nécessité accrue de communication et de coordination à l'intérieur des organismes

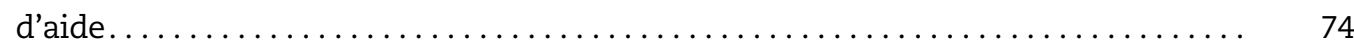

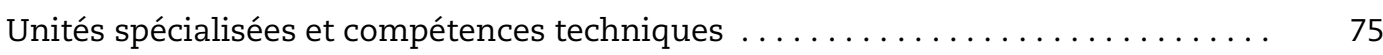

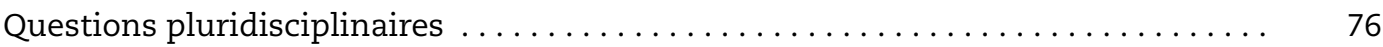

Chapitre 7. Gestion des ressources humaines de la coopération

pour le développement .......................... 79

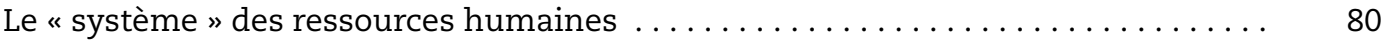

Planification des ressources humaines . . . . . . . . . . . . . . . . . . 80

Ressources humaines sur le terrain ......................... 86

Chapitre 8. Mise en œuvre de programmes bilatéraux dans les différents contextes des pays partenaires . . . . . . . . . . . . . . . 91

Mise en œuvre des partenariats pour le développement . . . . . . . . . . . . 92

Soutenir le développement dans les situations de partenariat difficile . . . . . . . 99

Notes.............................................. ${ }_{102}$

Chapitre 9. Aide humanitaire, dispositifs de cofinancement des ONG et autres

formes d'aide bilatérale. . . . . . . . . . . . . . . . . . . 103

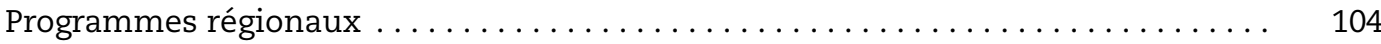

Programmes centralisés................................... 105

L'aide humanitaire . . . . . . . . . . . . . . . . . . . . . . . . . . . . . . . . . . . ${ }_{10} 108$

Cofinancement des ONG et des autres organismes de la société civile . . . . . . . 111

Chapitre 10. Aide multilatérale et contributions aux fonds mondiaux . . . . . . 115

Aide multilatérale . . . . . . . . . . . . . . . . . . . . . . . . . . . . . 116

Fonds mondiaux ........................................ ${ }^{2} 120$

Notes. . . . . . . . . . . . . . . . . . . . . . . . . . . . . . 122

Chapitre 11. Contrôles et contrepoids dans les systèmes de coopération au développement ............................ 123

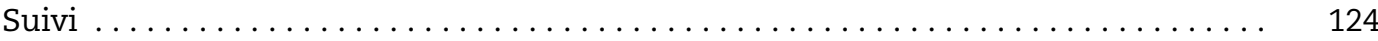

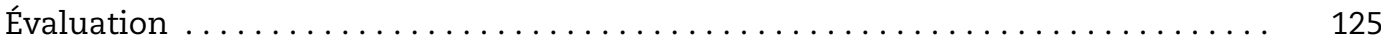

Gestion axée sur les résultats en termes de développement. . . . . . . . . . . 128

Intervention des instances nationales de contrôle des comptes. . . . . . . . . . 130

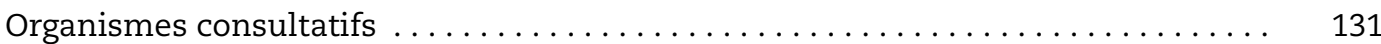

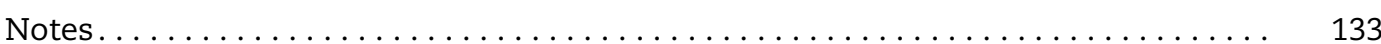

Annexe A.1. Profils de base des programmes d'aide exterieure des pays membres

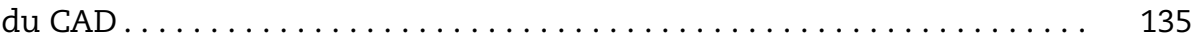

Annexe A.2. Rapport sur la gestion des programmes de coopération pour le développement au Mozambique ..................... 159

Annexe A.3. Les statistiques du CAD : introduction .................... 173 
Annexe A.4. Prise en compte de la réduction de la pauvretée et d'autres questions transversales : VIH/sida, égalité homme-femme et environnement ....

Annexe A.5. Gérer des projets et des programmes de développement $\ldots \ldots \ldots \ldots$.

Bibliographie

\section{Liste des encadrés}

1.1. Le rôle de la coopération pour le développement à l'aube du $\mathrm{XXI}^{\mathrm{e}}$ siècle $\ldots .$.

1.2. Principes relatifs à l'appropriation nationale, à l'harmonisation des pratiques

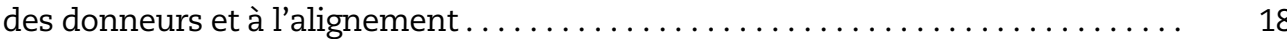

3.1. Donneurs non membres du CAD .............................. 41

3.2. Documents de référence publiés par le CAD .................. 43

4.1. Séminaire d'experts organisé par l'OCDE sur l'efficacité et la sélectivité de l'aide : intégrer de multiples objectifs dans le processus de répartition

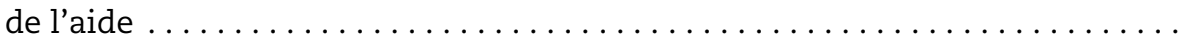

5.1. Restructuration des systèmes de coopération pour le développement des pays membres du CAD . . . . . . . . . . . . . . . . . . . . . .

5.2. Implication du ministère des Affaires étrangères dans la gestion

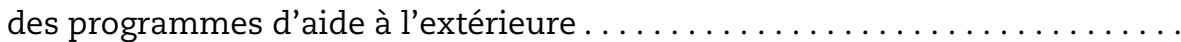

6.1. Principaux facteurs de succès de la prise en compte systématique des questions pluridisciplinaires . . . . . . . . . . . . . . . . . . 77

7.1. Mieux sous-traiter les services publics : principes directeurs et études de cas.... 84

8.1. Recommandations pratiques pour un bon fonctionnement des partenariats au service de la lutte contre la pauvreté

8.2. Recommandations pratiques pour de bonnes relations entre donneurs...... 94

8.3. La coopération entre donneurs au Mozambique et en Zambie............. 95

8.4. Cadre de développement national mis en place par l'ACDI en Haïti . . . . . . . . 101

9.1. Principes généraux et bonnes pratiques pour l'aide humanitaire . . . . . . . . 111

10.1. Programmes d'aide extérieure gérés par l'Union européenne. . . . . . . . . 118

11.1. Principes du CAD pour l'évaluation de l'aide au développement .......... 126

Liste des tableaux

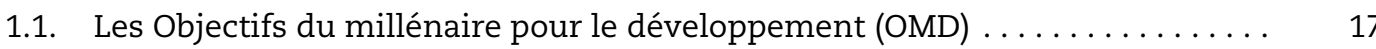

4.1. Budget consolidé de la coopération internationale (HGIS) $\ldots \ldots \ldots \ldots \ldots \ldots .48$

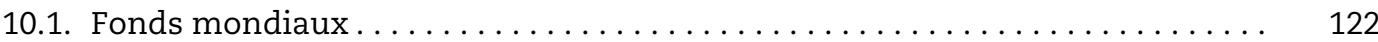

11.1. Principes appliqués par Danida pour adapter à différents publics les vecteurs du retour d'information

A3.1. Liste des bénéficiaires de l'aide établie par le CAD - 1 ${ }^{\mathrm{er}}$ janvier 2004....... 176

A5.1. Structure du cadre logique . . . . . . . . . . . . . . . . . . . . . . . . . . 191

A5.2. Exemple de cadre logistique - Création d'AGR pour les femmes . . . . . . . . . 193

Liste des graphiques

3.1. Aide publique au développement nette en $2003 \ldots \ldots \ldots \ldots \ldots \ldots \ldots \ldots \ldots$

3.2. Vue d'ensemble de l'aide extérieure distribuée par les membres du CAD .... 38

6.1. Agence australienne pour le développement international (AusAID),

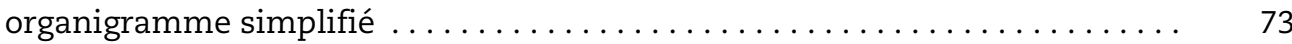

7.1. Importance du programme et effectifs professionnels au Mozambique . .... 87 
7.2. Montant géré par agent professionnel au Mozambique ............ 87

11.1. Du niveau des OMD au niveau indviduel : stratégie et organisation du DFID. . . . . . . . . . . . . . . . . . . . . . . . . . . . 130

A.2.1. Importance des programmes et importance des effectifs au Mozambique ..... 165

A.2.2. Programmes gérés au Mozambique, par professionnel .............. 166 


\section{Sigles}

ACIAR* Centre australien pour la recherche agricole internationale

AAD Agence autrichienne de développement

ACDI Agence canadienne de développement international

ACP Afrique, Caraibes et Pacifique

AECI Agence espagnole pour la coopération internationale

AFD Agence française de développement

APAD Agence portugaise de soutien du développement

APD Aide publique au développement

APEC* Coopération économique de la région Asie-Pacifique

AusAID* Agence australienne pour le développement international

BafD Banque africaine de développement

BasD Banque asiatique de développement

BERD Banque européenne pour la reconstruction et le développement

BID Banque interaméricaine de développement

BMZ* $^{*} \quad$ Ministère de la Coopération économique (Allemagne)

BVG Bureau du vérificateur général du Canada

CAD Comité d'aide au développement

CAP* Procédure d'appel global

CEE-ONU Commission économique pour l'Europe

CICID Comité interministériel de la coopération internationale et du développement (France)

CICR Comité international de la Croix-Rouge

CRDI Centre (canadien) de recherche pour le développement international

CSLP Cadre stratégique de lutte contre la pauvreté

CTB Coopération technique belge

DCD* Direction (irlandaise) de la coopération pour le développement

DDC Direction du développement et de la coopération (Suisse)

DED* Service allemand du développement

DEG* Société allemande d'investissement et de développement

DFID* Département (britannique) du développement international

DGCID Direction générale (française) de la Coopération internationale et du développement

DGCS* Direction générale (italienne) de la coopération internationale pour le développement

DGDC Direction générale (belge) de la coopération internationale

DGIS* Direction générale (néerlandaise) pour la coopération internationale

DTS Droits de tirage spéciaux

ECHO* Office humanitaire de la Communauté européenne

ECOSOC* Conseil économique et social des Nations unies

EES Évaluation environnementale stratégique

EIE Évaluation d'impact sur l'environnement

FED Fonds européen de développement 
FEM Fonds pour l'environnement mondial

FIDA Fonds international de développement agricole

FMI Fonds monétaire international

FNUAP Fonds des Nations unies pour la population

FRPC Facilité pour la réduction de la pauvreté et pour la croissance

GAVI* Alliance mondiale pour les vaccins et la vaccination

GC Groupe consultatif (Banque mondiale)

GFATM $^{*} \quad$ Fonds mondial de lutte contre le SIDA, la tuberculose et le paludisme

GTZ* Agence (allemande) pour la coopération technique

HCR Haut Commissariat des Nations unies pour les réfugiés

ICP* Institut de la coopération portugaise

IDA* Association internationale de développement

IPAD* Institut pour l'aide au développement du Portugal

JBIC* Banque japonaise pour la coopération internationale

JICA* Agence japonaise de coopération internationale

$\mathrm{KfW}^{*} \quad$ Banque allemande pour la reconstruction

MOPAN* Réseau sur l'évaluation des performances des organisations multilatérales

NEPAD Nouveau partenariat pour le développement de l'Afrique

NORAD Agence norvégienne de coopération pour le développement

NZAID Agence néo-zélandaise de coopération pour le développement

OCDE Organisation de coopération et de développement économiques

OMC Organisation mondiale du commerce

OMD Objectifs du Millénaire pour le développement

OMS Organisation mondiale de la santé

ONG Organisation non gouvernementale

ONUSIDA Programme commun des Nations unies sur le VIH/SIDA

PAM Programme alimentaire mondial

PIB Produit intérieur brut

PNUD Programme des Nations unies pour le développement

PNUE Programme des Nations unies pour l'environnement

PPTE Pays pauvres très endettés

PSA Partenariat stratégique pour l'Afrique

PSA* Public Service Agreement (Royaume-Uni)

RNB Revenu national brut

SAARC* Association de coopération régionale d'Asie du Sud

SADC* Communauté du développement de l'Afrique australe

SDA Service Delivery Agreement (Royaume-Uni)

SECIPI* Secrétariat d'État à la Coopération internationale et pour l'Amérique latine (Espagne)

SECO* Secrétariat d'État à l'Économie (Suisse)

Sida* Agence suédoise de développement international

SLP Stratégie de lutte contre la pauvreté

SNPC Système de notification des pays créanciers

UNICEF* Fonds des Nations unies pour l'enfance

UNIFEM $^{*}$ Fonds de développement des Nations unies pour la femme

USAID* United States Agency for International Development

USD Dollars des États-Unis

ZSP* Zone de solidarité prioritaire (France)

* Sigle dans la langue d'origine 


\section{Introduction}

$L^{\prime}$

objet du présent rapport est d'enrichir les connaissances sur les différentes pratiques et les structures parfois complexes adoptées par les 22 pays membres du Comité d'aide au développement (CAD) de l'OCDE ${ }^{1}$ pour gérer l'aide qu'ils accordent aux pays en développement et aux pays en transition. Ce document met en lumière les travaux pertinents menés au sein du CAD ainsi que les bonnes pratiques ou les caractéristiques notables des programmes de certains pays membres. On trouvera dans les annexes des informations sur les programmes des membres du $C A D$, les statistiques du CAD, la prise en compte systématique des questions transversales et la gestion des projets et programmes pour le développement. Le présent rapport s'inspire d'une étude similaire publiée par l'OCDE en $1999^{2}$.

La taille et l'efficacité des programmes de développement sont au centre des préoccupations du CAD depuis qu'il a été créé il y a 40 ans. Les examens de l'aide qu'il réalise contribuent de façon non négligeable à l'évaluation des performances de chacun de ses membres. C'est là un aspect essentiel du souci plus général d'améliorer l'efficacité de l'aide, notamment en évaluant l'impact global des programmes d'aide sur les pays partenaires ou sur certains secteurs tels que la santé ou l'éducation.

Présenter et améliorer les résultats constituent un problème de taille pour les membres du CAD. Le fait qu'au moins neuf pays membres aient d'une manière ou d'une autre procédé à une profonde réorganisation de leur aide au cours des dix dernières années témoigne des efforts entrepris pour résoudre une partie de ces problèmes par des réformes organisationnelles. Parmi les principales difficultés auxquelles les pays membres du CAD sont confrontés dans le domaine de la gestion de l'aide et qui sont abordées dans le présent rapport, on peut citer les suivants :

- Mettre en place un cadre juridique et administratif approprié pour le programme de développement.

- Sensibiliser davantage le grand public aux problèmes de développement et mieux les lui faire connaître.

- Maintenir et accroître les niveaux de financement et améliorer la prévisibilité des volumes d'aide.

- Affecter les fonds de l'aide au développement de manière appropriée, notamment entre les voies multilatérales et bilatérales.

- Améliorer la coordination avec les autres parties prenantes.

- Promouvoir la cohérence des politiques.

- Gérer les ressources humaines affectées à l'aide au développement.

- Mettre sur pied une structure organisationnelle efficace.

- Assurer le suivi et l'évaluation du programme et le soumettre à un examen indépendant.

- Promouvoir le partenariat et la décentralisation. 
La présente étude fait fonds sur les informations et données obtenues dans le cadre des examens en cours des politiques et programmes de coopération pour le développement des membres du CAD. En outre, afin de collecter des informations sur la gestion de l'aide sur le terrain, une mission a été organisée au Mozambique, l'un des pays les moins avancés au monde où 19 membres du CAD œuvrent activement et sont présents sur le terrain. Par ailleurs, une mission effectuée en République tchèque a permis de mieux comprendre les problèmes auxquels est confronté un pays non membre du CAD.

\section{Notes}

1. Les 22 pays membres du Comité d'aide au développement (CAD) sont actuellement : l'Allemagne, l'Australie, l'Autriche, la Belgique, le Canada, le Danemark, l'Espagne, les États-Unis, la Finlande, la France, la Grèce, l'Irlande, l'Italie, le Japon, le Luxembourg, la Norvège, la Nouvelle-Zélande, les Pays-Bas, le Portugal, le Royaume-Uni, la Suède et la Suisse. La Commission des Communautés européennes fait aussi partie des membres du CAD.

2. OCDE (1999), Comparaison des systèmes de gestion de la coopération au développement dans les pays de l'OCDE membres du CAD, OCDE, Paris. Voir : www.oecd.org/dataoecd/40/28/2094873.pdf. 


\section{Chapitre 1}

\section{Coopération pour le développement et nouveau millénaire : contexte, problèmes et solutions}

L'expérience montre que les pays en développement sont capables de sortir de la pauvreté et d'améliorer la qualité de vie de leur population. Mais la promotion du développement et la lutte contre la pauvreté n'en demeurent pas moins des tâches d'envergure. L'efficacité des efforts que ces pays déploient dans ce sens peut être accrue par l'aide extérieure. La Déclaration du millénaire, adoptée en 2000, prévoit la mise en place d'un partenariat en faveur du développement qui s'inspire de la stratégie définie par le CAD en 1996 sous le titre Le rôle de la coopération pour le développement à l'aube $\mathrm{du} \mathrm{XxI}^{\mathrm{e}}$ siècle. Les principes fondamentaux sur lesquels l'attention se porte désormais en priorité concernent l'appropriation par les pays partenaires de leur processus de développement, l'harmonisation des pratiques des donneurs et leur alignement sur les stratégies locales. La cohérence des politiques des donneurs au regard du développement fait l'objet d'un examen de plus en plus minutieux, puisque l'on sait maintenant quel peut être l'impact de ces politiques dans un monde fortement interdépendant. Le CAD est à la pointe du débat sur toutes ces questions, ses pays membres poursuivant leurs efforts pour améliorer le volume de leur aide, ainsi que le ciblage, l'efficacité et l'efficience des programmes qu'ils appliquent dans ce domaine. 


\section{La nécessité de faire face résolument aux problèmes de développement qui se posent aujourd'hui}

L'expérience acquise au cours des dernières décennies nous a appris que les pays pouvaient se sortir de la pauvreté et aider leurs populations à améliorer leur qualité de vie. Dans de nombreux pays, l'espérance de vie et les taux d'inscription dans l'enseignement primaire ont augmenté tandis que les taux de mortalité infantile reculaient. Des institutions favorables à la démocratie et à l'économie de marché ont été créées ou renforcées. Des innovations scientifiques ont contribué à améliorer la production agricole. L'Ouganda, le Sénégal et la Thaïlande ont particulièrement bien réussi à lutter contre l'épidémie du sida. La Corée, Singapour et le Taipei chinois, notamment, qui ont eu besoin de l'aide étrangère dans le passé, mettent désormais en œuvre leurs propres programmes de coopération pour le développement.

Dans le même temps, les forts contrastes qui caractérisent le monde font de la promotion du développement et de la lutte contre la pauvreté une tâche majeure toujours urgente. Sur les six milliards de personnes qui peuplent notre planète, un milliard vivent dans les pays développés et gagnent les quatre cinquièmes des revenus mondiaux alors que, pour un milliard de personnes vivant dans les pays en développement ou en transition, la vie est une lutte car il faut survivre avec moins d'un dollar USD par jour. L'espérance de vie à la naissance est de 80 ans voire plus au Japon, en Suède et en Suisse mais de moins de 40 ans dans certains pays ravagés par la guerre ou la propagation du sida comme le Botswana, le Malawi, le Sierra Leone, la Zambie et le Zimbabwe. Le taux moyen de mortalité maternelle est de 21 décès pour 100000 naissances d'enfants vivants dans les pays développés mais de plus de 1500 décès au Burundi, au Tchad, en Éthiopie, au Rwanda, en Sierra Leone et au Soudan. Les taux d'inscription dans l'enseignement primaire sont inférieurs à $40 \%$ en Angola, au Burkina Faso, en République démocratique du Congo et au Niger. Dans certains pays, notamment en Afghanistan, au Cambodge, au Tchad et en Éthiopie, moins d'une personne sur trois a accès à de l'eau salubre ${ }^{1}$.

Une étude récente commandée par la Banque mondiale montre qu'une majorité écrasante de leaders d'opinion dans les pays développés comme dans les pays en développement est convaincue que la paix et la stabilité mondiales ne seront possibles que si un effort majeur est fait pour réduire la pauvreté dans le monde ${ }^{2}$. En outre, les projections démographiques donnent à penser qu'en 2050 le monde pourrait ne pas être stable et qu'il sera difficile d'assurer la stabilité si l'on ne prend pas de mesures résolues pour englober davantage les pays en développement dans une économie mondiale de plus en plus intégrée. Selon les projections actuelles, la population des pays développés comptera toujours environ un milliard d'individus en 2050 mais l'on assistera à un recul important de la population active. En revanche, la population des pays en développement devrait passer de cinq à sept milliards et l'on devrait voir exploser le nombre de personnes d'âge actif ${ }^{3}$. Si rien n'est fait, les disparités entre les conditions de vie dans les pays 
développés et les pays en développement risquent d'accentuer davantage les pressions sur les migrations internationales.

S'il est certain que les pays en développement et en transition ${ }^{4}$ sont responsables au premier chef de leur développement économique et social et de la bonne gestion des affaires publiques, l'aide étrangère peut démultiplier l'efficacité de leurs efforts ${ }^{5}$. L'une des solides conclusions des récents travaux de recherche est que l'aide réussit à promouvoir la croissance économique et donc à faire reculer la pauvreté 6 . Compte tenu de la persistance des problèmes de développement, l'aide étrangère, accompagnée d'une plus grande cohérence des politiques, demeurera déterminante pour la génération actuelle mais aussi pour les générations futures.

\section{Tracer la voie à suivre}

Les membres du CAD reconnaissent qu'il existe un impératif moral évident qui veut que l'on réagisse à l'extrême pauvreté et aux souffrances humaines dans le monde mais ils savent aussi qu'il est de leur propre intérêt d'accroître la prospérité ainsi que la stabilité et la sécurité dans les pays en développement ${ }^{7}$. Une illustration concrète nous en est donné par le fait que les contribuables des pays membres du CAD consacrent aux pays en développement et en transition plus de 50 milliards USD chaque année sous forme de dons, de prêts assortis de conditions favorables et d'assistance technique, soit plus 95 pour cent de l'aide étrangère totale accordée par l'ensemble des gouvernements. En outre, les pays en développement et en transition reçoivent des financements du secteur privé des pays industrialisés par le biais essentiellement d'investissements directs étrangers ou de dons de fondations philanthropiques privées et d'organisations non gouvernementales.

Une conception commune se fait jour depuis une dizaine d'années quant à la répartition de l'aide étrangère. On s'accorde à présent largement à reconnaître que, dans les pays où la situation macroéconomique est saine et où les institutions fonctionnent bien, l'aide devrait produire des résultats durables si elle vient à l'appui des stratégies de développement élaborées par les pays partenaires. Les fondements de ce paradigme sont énoncés dans le rapport de 1996 du CAD intitulé Le rôle de la coopération pour le développement à l'aube du XXI ${ }^{e}$ siècle $e^{8}$ (voir encadré 1.1). Ce rapport a aussi permis d'inaugurer une nouvelle ère où beaucoup de membres du CAD se soucient davantage des résultats obtenus du point de vue du développement que des moyens mis en œuvre et des activités réalisées individuellement. Depuis 1996, le CAD, le système des Nations unies et la communauté en développement dans son ensemble se sont efforcés de traduire cette vision des choses dans les faits et de la rendre opérationnelle. Des travaux ont aussi été entrepris pour déterminer les types d'approche à adopter pour soutenir le développement dans les pays où l'environnement général et la gestion des affaires publiques laissent à désirer.

L'adoption de la Déclaration du millénaire ${ }^{9}$ par 189 pays à l'Assemblée générale des Nations unies de septembre 2000 a joué un rôle déterminant en précisant la conception du développement présentée dans la stratégie intitulée Le rôle de la coopération pour le développement à l'aube du XXI ${ }^{e}$ siècle et en lui donnant une plus grande crédibilité. Dans cette déclaration, les pays en développement comme les pays développés ont approuvé l'établissement d'un partenariat favorable au développement et à l'éradication de la pauvreté. La déclaration énonce un ensemble d'objectifs et de cibles liés entre eux - les Objectifs du millénaire pour le développement (OMD) (voir tableau 1.1) - qui intègrent et 


\section{Encadré 1.1. Le rôle de la coopération pour le développement à l'aube du XXI ${ }^{\mathrm{e}}$ siècle}

Le rapport du CAD de 1996 intitulé Le rôle de la coopération pour le développement à l'aube du $\mathrm{XXI}^{\mathrm{e}}$ siècle définit un cadre d'action qui repose sur quatre grands piliers :

1. Une vision commune définie par des objectifs mesurables de bien-être économique, de développement social et de viabilité environnementale qui doivent être poursuivis pays par pays. parmi ces objectifs figurent celui consistant à réduire de moitié d'ici à 2015 la proportion de personnes dont le revenu est inférieur à moins d'un dollar USD par jour.

2. Un concept de partenariat véritable où des changements fondamentaux seraient menés à bien dans le cadre d'engagements réciproques de répartition des responsabilités, de renforcement de l'appropriation et des capacités locales, et, d'encouragement de la participation et de l'autonomie.

3. L'accent mis sur les fondements qualitatifs, tels que des pratiques démocratiques et responsables, la protection des droits de la personne et respect de l'État de droit, indispensables à la réalisation d'objectifs plus mesurables.

4. La nécessité d'une cohérence entre les politiques d'aide et les autres politiques qui ont une incidence sur les pays en développement.

développent les objectifs adoptés dans la stratégie sur Le rôle de la coopération pour le développement à l'aube $\mathrm{du} \mathrm{XXI}^{e}$ siècle.

Ces dernières années, les membres du CAD se sont efforcés d'œuvrer en respectant l'engagement pris dans ce dernier rapport, à savoir « ... changer [leur] manière de penser et d'agir et la coordination des efforts devra pour cela être bien plus étroite que ce n'a été le cas jusqu'à présent ». Les lignes directrices du CAD sur la lutte contre la pauvreté publiées en 2001 sont un aspect clé de cette nouvelle conception ${ }^{10}$. Elles montrent que les principes qui sous-tendent la stratégie sur le rôle de la coopération pour le développement à l'aube du XXI ${ }^{\mathrm{e}}$ siècle - c'est-à-dire le partenariat, l'appropriation, la conduite des opérations par le pays concerné, une large participation, l'efficacité du point de vue du développement et l'obligation de rendre des comptes - ont des conséquences de vaste portée sur la façon dont opèrent les organismes de développement. Ces derniers ont maintenant l'obligation de rendre des comptes aux pays partenaires et à leurs opinions publiques quant aux actions menées et aux engagements pris. Ils doivent mettre en place une collaboration bien plus étroite et coordonnée avec un plus large éventail de partenaires et jouer un rôle de soutien plutôt que de déclencheurs du développement. Les membres du CAD doivent aussi apporter une aide adaptée aux besoins et aux priorités du pays partenaire lorsque les conditions d'un partenariat sont remplies. Cette tâche a été facilitée par la récente émergence dans la plupart des pays à faible revenu des cadres stratégiques de lutte contre la pauvreté (CSLP). Ces documents, préparés par les gouvernements partenaires dans le cadre de processus participatifs auxquels sont associés la sociétés civile et d'autres partenaires du développement, servent de base à l'octroi de prêts assortis de conditions de faveur par la Banque mondiale et le Fonds monétaire international (FMI) depuis 1999 et aux opérations d'allègement de dettes au titre de l'Initiative pour le pays pauvres très endettés (PPTE). 


\section{Tableau 1.1. Les Objectifs du millénaire pour le développement (OMD)}

Objectifs et cibles tirés de la Déclaration du millénaire

Objectif 1: Réduire l'extrême pauvreté et la fin.

Cible 1: Réduire de moitié, entre 1990 et 2015, la proportion de la population dont le revenu est inférieur à un dollar par jour.

Cible 2: Réduire de moitié, entre 1990 et 2015, la proportion de la population qui souffre de la faim.

Objectif 2: Assurer l'éducation primaire pour tous.

Cible 3 : D'ici à 2015, donner à tous les enfants, garçons et filles, partout dans le monde, les moyens d'achever un cycle complet d'études primaires.

Objectif 3 : Promouvoir l'égalité des sexes et l'autonomisation des femmes.

Cible 4 : Éliminer les disparités entre les sexes dans les enseignements primaire et secondaire d'ici à 2015 si possible et à tous les niveaux de l'enseignement en 2015 au plus tard.

Objectif 4: Réduire la mortalité infantile

Cible 5: Réduire de deux tiers, entre 1990 et 2015, le taux de mortalité des enfants de moins de 5 ans.

Objectif 5 : Améliorer la santé maternelle.

Cible 6: Réduire de trois quarts, entre 1990 et 2015, le taux de mortalité maternelle.

Objectif 6: Combattre le VIH/sida, le paludisme et d'autres maladies.

Cible 7: D'ici à 2015, avoir stoppé la propagation du VIH/sida et commencé à inverser la tendance actuelle.

Cible 8 : D'ici à 2015, avoir maîtrisé le paludisme et d'autres grandes maladies, et avoir commencé à inverser la tendance actuelle.

Objectif 7: Assurer un environnement durable.

Cible 9: Intégrer les principes du développement durable dans les politiques nationales et inverser la tendance actuelle à la déperdition des ressources environnementales.

Cible 10: Réduire de moitié, d'ici à 2015, le pourcentage de la population qui n'a pas accès de façon durable à un approvisionnement en eau potable salubre.

Cible 11: Réussir, d'ici à 2020, à améliorer sensiblement la vie d'au moins 100 millions d'habitants de taudis

Objectif 8 : Mettre en place un partenariat mondial pour le développement.

Cible 12: Poursuivre la mise en place d'un système commercial et financier multilatéral ouvert, fondé sur des règles, prévisible et non discriminatoire.

Cela suppose un engagement en faveur d'une bonne gouvernance, du développement et de la lutte contre la pauvreté, aux niveaux tant national qu'international.

Cible 13: S'attaquer aux besoins particuliers des pays les moins avancés.

La réalisation de cette cible suppose l'admission en franchise et hors contingents des produits exportés par les pays les moins avancés ; l'application du programme renforcé d'allègement de la dette des PPTE et l'annulation des dettes publiques bilatérales ; et l'octroi d'une APD plus généreuse aux pays qui démontrent leur volonté de lutter contre la pauvreté.

Cible 14 : Répondre aux besoins particuliers des petits États insulaires en développement.

(en appliquant le Programme d'action pour le développement durable des petits États insulaires en développement et les conclusions de la vingt-deuxième session extraordinaire de l'Assemblée générale).

Cible 15: Traiter globalement le problème de la dette des pays en développement, par des mesures d'ordre national et international propres à rendre leur endettement viable à long terme.

Cible 16: En coopération avec les pays en développement, formuler et appliquer des stratégies qui permettent aux jeunes de trouver un travail décent et utile.

Cible 17: En coopération avec l'industrie pharmaceutique, rendre les médicaments essentiels disponibles et abordables dans les pays en développement.

Cible 18: En coopération avec le secteur privé, faire en sorte que les avantages des nouvelles technologies, en particulier des technologies de l'information et de la communication, soient accordés à tous.

La création du Groupe d'étude sur les pratiques des donneurs en 2000 fait partie des mesures importantes prises par le CAD. L'objectif de ce Groupe, aux travaux duquel participent des donneurs multilatéraux et des pays en développement, est de contribuer au renforcement de l'appropriation locale en mettant en évidence, sur la base d'éléments concrets, les pratiques susceptibles de réduire de manière efficace par rapport au coût les ponctions exercées sur les capacités de gestion de l'aide des pays partenaires et les coûts 
de transaction y afférents. Le Groupe d'étude a également établi un ensemble de documents sur les bonnes pratiques ${ }^{11}$. Il a aussi apporté une importante contribution aux efforts internationaux entrepris pour promouvoir l'harmonisation des politiques, procédures et pratiques opérationnelles des donneurs avec les systèmes des pays partenaires ; ses travaux ont abouti à la Déclaration de Rome sur l'harmonisation ${ }^{12}$, approuvée au Forum de haut niveau de 2003. Grâce à cela, un accord a pu se faire sur un ensemble de principes dans les domaines de l'appropriation, de l'harmonisation des pratiques des donneurs et de l'alignement (voir encadré 1.2).

Plusieurs réunions ministérielles et sommets importants organisés ces dernières années ont également renforcer l'attachement à un partenariat de vaste portée axé sur les résultats pour promouvoir le développement international. La plus marquante de ces manifestations est la conférence internationale sur le financement du développement tenue à Monterrey en 2002.

\section{Encadré 1.2. Principes relatifs à l'appropriation nationale, à l'harmonisation des pratiques des donneurs et à l'alignement}

Grâce en partie aux travaux auxquels le $\mathrm{CAD}$ a participé, la communauté du développement s'est entendue sur un certain nombre de principes dans les domaines de l'appropriation nationale, de l'harmonisation et de l'alignement. Ces principes dictent aux agences de développement de :

- Appuyer les priorités, objectifs et résultats propres aux pays partenaires. Ceci implique l'alignement sur la stratégie nationale (une stratégie nationale de réduction de la pauvreté ou son équivalent, avec prise en compte au niveau national des Objectifs $d u$ millénaire pour développement, le cas échéant) et l'utilisation de procédures et systèmes nationaux fiables (notamment le budget de l'État, le cycle de notification et le calendrier de suivi).

- Coordonner les opérations avec celles des autres organismes d'aide sous la supervision $\mathrm{du}$ pays partenaire et encourager les actions conjointes chaque fois que possible (notamment la coopération déléguée, un bailleur de fonds agissant pour le compte d'un autre).

- Renforcer les institutions, les systèmes et les capacités propres aux pays partenaires pour planifier et mettre en œuvre les projets et les programmes, faire rapport des résultats et évaluer leur processus de développement et leurs réalisations, en évitant les mécanismes parallèles conçus par les bailleurs de fonds.

- Lors de la Conférence internationale des Nations unies sur le financement du développement tenue à Monterrey en 2002, les pays développés et les pays en développement ont conclu un accord pour éradiquer la pauvreté, parvenir à une croissance économique soutenue et promouvoir le développement durable. Les participants se sont engagés à mettre en œuvre un nouveau partenariat entre pays développés et pays en développement afin de réaliser les objectifs pour le développement convenus à l'échelon international, notamment ceux contenus dans la Déclaration du millénaire. Dans le Consensus de Monterrey, les pays se sont engagés à poursuivre des politiques saines, à promouvoir la bonne gouvernance à tous les niveaux et le respect de l'État de droit. Ils se sont aussi engagés à mobiliser les ressources 
nationales, attirer les investissements internationaux, promouvoir le commerce international en tant que moteur du développement, accroître la coopération technique et financière internationale au service du développement, augmenter le financement durable de la dette et les allègements de la dette extérieure, et renforcer la cohérence des systèmes, monétaires, financiers et commerciaux internationaux.

- Le Sommet mondial pour le développement durable tenu à Johannesburg en 2002 a confirmé que des progrès importants avaient déjà été réalisés sur la voie d'un consensus et d'un partenariat à l'échelle mondiale pour tous suivent la même voie conduisant à un monde soucieux du développement durable. Les participants ont souligné que l'écart de plus en plus grand entre les pays développés et les pays en développement était une menace majeure pour la prospérité, la sécurité et la stabilité mondiale. Selon le Plan d'action convenu lors de ce Sommet, l'éradication de la pauvreté est la principale tâche à laquelle le monde doit faire face et elle est indispensable au développement durable, en particulier dans les pays en développement. Les participants se sont accordés à penser que des mesures concertées et concrètes devaient être prises par toutes les parties intéressées pour permettre aux pays en développement de réaliser leurs objectifs en matière de développement.

- La quatrième Conférence ministérielle de l'Organisation mondiale du commerce (OMC) tenue à Doha en 2001 a lancé une nouvelle série de négociations commerciales multilatérales. Comme cette dernière est axée sur des questions importantes pour les pays en développement, par exemple comment améliorer l'accès de leurs produits agricoles au marché, elle est connue sous le nom de Programme de Doha pour le développement. La principale tâche de la cinquième Conférence ministérielle de l'OMC organisée à Cancún en 2003 était de faire le point sur l'état d'avancement de ces négociations et d'autres travaux en cours. Cette réunion a abouti à une impasse mais les consultations se poursuivent en vue de trouver les moyens de faire avancer les négociations.

Le consensus auquel on est parvenu par le biais du rapport sur Le rôle de la coopération pour le développement à l'aube du XXI siècle, la Déclaration du millénaire et le Consensus de Monterrey sur les priorités du développement, les besoins en ressources et l'accès accru à des financements extérieurs a créé une importante dynamique. Cette dernière, associée à l'initiative PPTE et à l'élaboration de stratégies nationales de lutte contre la pauvreté, ainsi que l'accord général sur les objectifs et approches idéales d'acheminement de l'aide étrangère, marquent un tournant dans l'histoire de la coopération pour le développement et créent de solides fondations pour l'avenir.

Cependant, il n'y a pas lieu de se féliciter car les problèmes de développement qui restent sont importants. Les données réunies par l'OCDE montrent que pour chacun des objectifs et chacune des cibles figurant dans la Déclaration du millénaire, des signes encourageants de progrès peuvent être observés dans certaines régions du monde alors que dans d'autres on trouve des signes préoccupants de stagnation ou de recul ${ }^{13}$. Les pays les moins avancés ${ }^{14}$, en particulier les pays d'Afrique subsaharienne, éprouvent de graves difficultés dans leur tentative de réaliser les objectifs du millénaire pour le développement. Il est donc essentiel que, individuellement et collectivement, les programmes d'aide étrangère des pays membres du CAD soient rigoureusement gérés et soigneusement ciblés et qu'ils se complètent les uns les autres de manière à renforcer leur efficacité et leur impact. 


\section{La cohérence des politiques au service du développement}

Pour importante que soit l'aide étrangère, dans un monde fortement intégré il y a bon nombre de domaines où les politiques des gouvernements donneurs peuvent compléter ou au contraire contrarier les efforts de développement des pays partenaires. Par exemple, les subventions qui sont accordées au niveau national au secteur de l'agriculture dans les pays industrialisés peuvent avoir un effet de distorsion des échanges, les restrictions aux importations d'ordre écologique et sanitaire peuvent agir comme des obstacles non tarifaires et les politiques d'immigration peuvent priver les pays en développement de leurs travailleurs en bonne santé et de leurs diplômés formés grâce aux programmes d'aide étrangère. Cependant, les politiques des pays industrialisés peuvent aussi stimuler les échanges et les investissements et faciliter le transfert de technologie. Tout ceci montre combien il importe que les gouvernements donnent suite à leur engagement de promouvoir le développement international en tenant compte de la dimension développement lorsqu'ils élaborent leurs politiques dans un large éventail de domaines ${ }^{15}$. S'ils ne le font pas, l'aide étrangère risque d'avoir simplement pour effet de contrebalancer les coûts imposés aux pays partenaires par l'absence de cohérence des politiques des pays donneurs. Consciente des conséquences d'un monde de plus en plus intégré tant pour les pays développés que pour les pays en développement, la Suède a récemment adopté une législation qui encourage l'adoption d'une approche associant l'ensemble de l'administration à la promotion du développement international (voir ci-après).

\section{Un exemple : la Suède}

Le Parlement suédois a adopté une législation en décembre 2003 qui fait de la promotion d'un développement équitable et durable dans le monde un objectif pour l'ensemble des domaines d'intervention des autorités suédoises. Shared responsibility: Sweden's policy for global development (réf. : 2002/03:122) jette donc les fondements d'un processus de prise de décision de nature à promouvoir le développement international. La législation souligne qu'il est essentiel d'instaurer une étroite collaboration avec les acteurs dans l'ensemble des secteurs de la société, notamment les autorités locales, les institutions de la société civile, le secteur privé et les syndicats. Il convient de noter que les échanges, l'agriculture, l'environnement, la sécurité, les migrations et la politique économique figurent parmi les exemples de domaines où les politiques peuvent et doivent soutenir le développement mondial. La coopération pour le développement elle-même est considérée comme un aspect de la politique générale de la Suède. La nouvelle politique devrait entraîner quelques changements dans l'organisation et la gestion de l'Agence suédoise chargée de la coopération pour le développement.

L'expérience des pays membres du CAD donne à penser que la promotion de la cohérence des politiques au service du développement ${ }^{16}$ peut être un processus difficile en raison d'intérêts nationaux concurrents. Bien que la parfaite cohérence des politiques en faveur du développement puisse être un objectif impossible à atteindre, il est essentiel que les décideurs tiennent pleinement compte de l'impact et des conséquences potentiels des décisions prises sur les pays en développement. Les travaux menés au sein du CAD, s'appuyant sur des rapports plus généraux entrepris à l'OCDE ${ }^{17}$, mettent en lumière 
quelques stratégies institutionnelles susceptibles de favoriser la cohérence des politiques, notamment :

- Ferme volonté politique : afficher clairement et officiellement l'objectif de réduction de la pauvreté, témoin d'un fondement solide sur lequel bâtir les efforts du gouvernement en vue d'assurer que les problèmes des pays en développement soient pris en compte lors de l'élaboration des politiques.

- Mécanismes de coordination à l'échelle de l'ensemble de l'administration : mettre en place des dispositifs interministériels formels et informels au niveau politique et à celui des hauts fonctionnaires afin de passer au crible les politiques et décisions au regard des objectifs de réduction de la pauvreté. Il importe que les organismes d'aide au développement fassent partie de ces mécanismes et ne soient pas représentés par une autre institution ou un autre ministère susceptible d'avoir un point de vue différent sur les problèmes considérés.

- Capacité analytique: doter les organismes d'un personnel ayant la formation, l'expérience et le temps nécessaire pour évaluer les problèmes généraux du point de vue de leurs conséquences effectives ou potentielles sur les pays en développement et la lutte contre la pauvreté. Ces agents peuvent être regroupés au sein d'une unité consacrée à la cohérence des politiques à l'intérieur de l'organisme d'aide (voir ci-après).

\section{Un exemple : les Pays-Bas}

Pour contribuer à la promotion de la cohérence des politiques au service du développement, les Pays-Bas ont créé en 2002 une petite Unité de la cohérence des politiques placée sous la supervision d'un directeur au sein de la direction générale pour la coopération pour le développement du ministère des affaires étrangères. Cette Unité a deux fonctions principales : i) participer à l'élaboration des politiques dans les domaines ne concernant pas le développement et ii) s'occuper de cas concrets d'incohérence des politiques. Comme beaucoup de problèmes ayant une incidence sur les pays en développement entrent dans le champ de compétence de l'Union européenne, cette Unité participe au dispositif de coordination de cette dernière aux Pays-Bas et s'emploie à coaliser les États membres de l'Union européenne qui partagent ses conceptions. La stratégie de l'Unité s'agissant de lutter contre l'incohérence des politiques est davantage opportuniste de caractère : elle se saisit des occasions qui se présentent pour promouvoir le changement. Par exemple, en 2002, l'Unité a profité de l'examen à moyen terme de la politique agricole commune de l'Union européenne et des négociations menées au sein de l'OMC sur le Programme de Doha pour préparer un rapport sur le renforcement de la cohérence des politiques menées dans les domaines de l'agriculture, des échanges et du développement, s'agissant notamment des trois produits bénéficiant de fortes subventions que sont le coton, le riz et le sucre. Le texte ainsi mis au point, intitulé Memorandum on Coherence between Agricultural and Development Policy, a été signé à la fois par le ministre de l'Agriculture, de la Gestion de l'Environnement et de la Pêche et par le ministre de la Coopération pour le Développement, approuvé au niveau du gouvernement et soumis au Parlement.

Des enseignements peuvent être tirés de l'expérience des membres du CAD concernant le renforcement de la cohérence des politiques au service du développement. 
L'éventail des questions, acteurs et mesures en cause donne à penser qu'aucun système d'élaboration des politiques ne peut garantir une plus grande cohérence des politiques dans tous les contextes. Il faut donc s'attendre à ce que diverses approches soient adoptées et à ce que, dans son contexte national spécifique, chacune soit tout aussi efficace que les autres pour promouvoir la cohérence des politiques. On constate sans surprise que le degré de cohérence des politiques tend à diminuer lorsqu'un domaine d'action est sensible au plan intérieur et qu'il existe des groupes d'intérêts nationaux forts et que les organismes publics ont d'autres priorités. Il arrive que des mesures positives soient prises mais pas nécessairement en vue de promouvoir la cohérence des politiques au service du développement. Par exemple, la pression exercée pour réformer la politique agricole commune de l'Union européenne a pour l'essentiel été le fait de contributeurs nets au programme, d'où la question de savoir si l'absence de cohérence des politiques au service du développement suffit à justifier un changement de politique. Cela montre que les organismes d'aide au développement ont intérêt à nouer des alliances stratégiques avec d'autres parties prenantes de manière à encourager des réformes susceptibles de contribuer au renforcement de la cohérence des politiques au service du développement.

\section{Notes}

1. Banque mondiale (2003), World Development Indicators, Banque mondiale, Washington. Il convient de noter qu'il n'existe pas de données publiées pour tous les pays en développement.

2. Princeton Survey Research Associates (2003), Global Poll: Multinational Survey of Opinion Leaders 2002. Washington. Cette étude porte sur 2600 leaders d'opinion dans 48 pays.

3. Division de la population de l'ONU (2003), World Population Prospects: The 2002 Revision, New York. Les chiffres indiqués sont ceux qui ressortent des scénarios intermédaires.

4. Dans la terminologie du OCDE, les « pays et territoires en développement » sont ceux qui figurent dans la première partie de la Liste des bénéficiaires de l'aide établie par le CAD tandis que les " pays et territoires en transition » figurent dans la deuxième partie de cette Liste. (Voir l'annexe 3 pour plus de précisions.)

5. Dans le présent rapport, les mots " coopération pour le développement ", " aide étrangère " et " aide » sont interchangeables et renvoient à l'aide au développment et à l'aide publique.

6. McGillivray, M. (2003), Aid Effectiveness and Selectivity: Integrating Multiple Objectives into Aid Allocations, World Institute for Development Economics Research Discussion Paper n²003/71, Helsinki.

7. OCDE (1996), Le rôle de la coopération pour le développement à l'aube du XXI eiècle, OCDE, Paris.

8. Voir : www.oecd.org/dataoecd/23/35/2508761.pdf.

9. Voir : www.un.org/millennium/declaration/ares552e.pdf. L'Assemblée générale a demandé au Secrétaire général des Nations unies de préparer un rapport annuel sur les progrès accomplis par le système des Nations unies et les pays membres sur la voie de la mise en œuvre de la Déclaration du millénaire. Cette information peut être obtenue sur Internet à l'adresse suivante : www.un.org/millenniumgoals/.

10. Voir : www.oecd.org/dataoecd/47/14/2672735.pdf.

11. OCDE (2003), Lignes directrices et ouvrages de référence du CAD : Harmoniser l'aide pour renforcer son efficacité, OCDE, Paris. Voir : www.oecd.org/dataoecd/0/48/20896122.pdf.

12. Voir : www1.worldbank.org/harmonization/romehlf/Documents/RomeDeclaration.pdf.

13. OCDE (2004), "Progrès accomplis vers les objectifs du millénaire pour le développement ", Les dossiers du CAD, vol. 5, n 1, OCDE, Paris, pp. 58 à 80.

14. L'expression " pays les moins avancés " renvoie aux pays les plus pauvres du monde selon une classification de l'ONU fondée sur des critères de revenu, de diversification économique, de développement social et de démographie. 
15. Parmi d'autres engagements, les pays développés se sont fixés pour objectif de veiller à ce que l'ensemble des politiques pertinentes soient cohérentes avec les objectifs du développement et ne les compromettent pas. OCDE (1996), Le rôle de la coopération pour le développement à l'aube du $\mathrm{XXI}^{e}$ siècle, OCDE, Paris. Voir également: Pour un programme d'action commun de l'OCDE au service du développement - Communiqué final de la réunion au niveau des ministres 2002.

16. Il convient de faire la distinction entre cohésion des politiques, c'est-à-dire veiller à ce que les politiques de coopération pour le développement ne soient pas contradictoires entre elles et cohérence des politiques qui implique la poursuite d'objectifs de développement par le biais de politiques d'aide et autres se renforçant mutuellement.

17. OCDE (1996), Assurer la cohérence des politiques : instruments et contraintes, études hors série sur la gestion publique, $n^{\circ} 12$, OCDE, Paris. 



\section{Chapitre 2}

\section{Les fondements juridiques et politiques de la coopération pour le développement}

Plus de la moitié des pays membres du CAD ont adopté des lois qui jettent les bases de leur programme de coopération pour le développement et en fixent les grands objectifs, et les deux tiers ont élaboré des déclarations d'orientation qui, si elles n'ont pas force de loi, énoncent les priorités et les modalités d'acheminement de l'aide. Il est essentiel que la coopération pour le développement dispose d'une base juridique et politique solide et qu'elle soit représentée au gouvernement si l'on veut que les objectifs en matière de développement soient considérés comme un élément important de la stratégie du gouvernement dans l'ensemble de ses grands domaines d'intervention et si l'on veut ces objectifs soient prioritaires. Bien que la coopération pour le développement bénéficie d'un soutien public important dans les pays membres du CAD, les problèmes de développement ne sont pas toujours bien compris par l'opinion. Compte tenu de l'importance de la contribution des citoyens à la coopération pour le développement, il est indispensable que ceux-ci comprennent mieux les enjeux du développement si les pays veulent honorer l'engagement pris d'accroître les niveaux de l'aide au développement et de promouvoir la cohérence des politiques. 


\section{La base législative}

La base législative des programmes de coopération pour le développement reflète dans une large mesure la tradition juridique de chaque pays membre du CAD. Il est frappant de constater que si 12 pays membres (voir annexe A.1), en particulier ceux qui ont un système juridique codifié (notamment l'Espagne, voir ci-après), disposent d'une législation détaillée pour orienter leurs programmes d'aide étrangère, les autres pays membres du CAD n'ont aucune législation, les politiques et stratégies globales étant définies par le gouvernement et l'autorisation de dépenser étant obtenue par le biais de la loi de crédit. Dans quelques pays membres du CAD, les grandes lignes du programme de coopération sont énoncées dans la législation ordinaire mais la mise en œuvre des activités, dans les limites fixées par la loi, est déléguée au gouvernement ou au ministre compétent. Une base législative solidement élaborée présente l'avantage de la transparence et de la clarification des responsabilités entre les différentes instances gouvernementales susceptibles d'intervenir, et elle permet de faire des objectifs de développement le principal axe de l'aide au développement dans l'ensemble du système. En revanche, les pays dont la base législative est moins formalisée ont une plus grande marge de manœuvre pour agir, ce qui peut être un avantage lorsqu'il s'agit de s'assurer la coopération des organismes de développement et d'autres instances gouvernementales dont les politiques et actions ont une incidence sur les perspectives de développement des pays en développement.

\section{Un exemple : l'Espagne}

Le Parlement espagnol a adopté une nouvelle loi importante en 1998 afin de mettre à jour et de renforcer les fondements législatifs du programme de coopération pour le développement du pays. La loi 23/198, en date du 7 juillet, sur la coopération internationale pour le développement contient six chapitres. Le chapitre I définit la politique espagnole de coopération internationale dans le domaine du développement. Le chapitre II présente dans leurs grandes lignes la planification, les instruments et les modalités de la politique espagnole en matière de coopération internationale. Le chapitre III porte création des institutions chargées de l'élaboration et de la mise en œuvre de la politique. Le chapitre IV concerne les ressources, le chapitre $\mathrm{V}$ les questions de personnel et le chapitre IV la participation des ONG et des autres partenaires de la société civile. Aux termes de la loi, l'Agence espagnole de coopération internationale est l'organisme chargé de mettre en œuvre la politique de l'Espagne dans ce domaine. Les statuts de l'Agence (rôle et responsabilités, buts et fonctions, organes de gestion, structures institutionnelles de base, politique de gestion des ressources humaines, dispositions concernant les actifs ainsi que les questions financières et économiques, contrats de travail) ont été approuvés par le Décret Royal 3432/2000, en date du 15 décembre. 


\section{Le contexte politique}

Les buts et objectifs énoncés dans la Déclaration du millénaire fixent à l'aide étrangère internationale des objectifs à moyen terme. Cependant, il se peut que la réalisation d'objectifs en matière de développement ne soit pas l'unique motivation des donneurs. Beaucoup de membres du CAD admettent que la coopération pour le développement fait partie intégrante de leurs relations extérieures; pour certains, elle est même un instrument qui leur permet de poursuivre leurs intérêts nationaux. L'ancien directeur adjoint de USAID estime pour sa part que la plupart des gouvernements ont d'autres objectifs en tête, notamment diplomatiques, commerciaux, nationaux et culturels ${ }^{1}$. Selon le rapport de la Commission Simons sur l'aide étrangère de l'Australie, le programme d'aide peine à faire face au triple mandat qui lui a été confié, à savoir favoriser les retombées positives pour l'Australie sur le plan commercial et diplomatique et promouvoir l'intérêt des pays en développement ${ }^{2}$. La loi britannique sur le développement international (International Development Act, 2000) rend illégal les pratiques ayant d'autres buts que de faire reculer la pauvreté, comme celle consistant à promouvoir les intérêts commerciaux en liant l'aide à l'acquisition de biens et services britanniques.

L'équilibre approprié entre les objectifs du développement et les autres buts poursuivis dans le cadre des programmes d'aide relève en dernière analyse d'un choix politique qui revient à chaque membre du CAD. Toutefois, ce choix s'inscrit dans les limites de la définition officielle de l'aide publique au développement (APD), élaborée par le CAD et approuvée par tous ses membres, en vertu de laquelle le principal objectif de l'aide est de promouvoir le développement économique et le bien être des pays partenaires. En outre, les questions de développement doivent recevoir toute l'attention voulue car, pour obtenir des résultats sur le front du développement et conserver le soutien de l'opinion publique, il faut que l'aide étrangère favorise la réalisation des objectifs de développement. Par ailleurs, certaines initiatives internationales contribuent à réduire les possibilités d'utiliser l'aide étrangère pour d'autres buts que le développement. Il en va ainsi par exemple de la recommandation adoptée par le CAD en 2001 sur le déliement de la plupart des catégories d'APD aux pays ${ }^{3}$ les moins avancés qui vise à réduire les possibilités de faire de l'aide étrangère un instrument pour promouvoir des intérêts commerciaux.

\section{Les déclarations d'orientation}

Indépendamment de la base législative, les pays membres du CAD estiment souvent utile de préparer une déclaration d'orientation dans laquelle ils énoncent la finalité et les objectifs de leur aide étrangère. Par exemple, 20 pays membres du CAD ont adopté un objectif prioritaire qui guide leur programme de coopération pour le développement et la lutte contre la pauvreté occupe une place essentielle dans l'objectif global du programme d'aide d'au moins 16 membres (voir l'annexe A.1 pour un aperçu des objectifs et des déclarations d'orientation des membres du CAD). Les déclarations d'orientation peuvent prendre la forme d'un Livre blanc du gouvernement et doivent en principe être approuvées par tous les ministres chargées des activités ayant un impact sur les perspectives de développement des pays en développement. Dans la mesure où les politiques d'aide font souvent l'objet d'un débat avec la société civile ou tiennent compte des apports de celle-ci, le processus consultatif peut être aussi important que le document lui-même s'il contribue à la sensibilisation de l'opinion publique et mobilise le soutien de celle-ci en faveur du programme de coopération pour le développement. Les déclarations d'orientation peuvent aussi assurer une unité de vue dans les cas où plusieurs organismes sont chargés de la 
mise en œuvre de l'aide étrangère, comme en Allemagne par exemple (voir ci-après). Enfin, ces déclarations de principe contiennent des informations qui ne peuvent pas toujours faire l'objet d'une loi, par exemple les ambitions concernant les niveaux futurs de l'APD en pourcentage du revenu national brut (RNB). Il arrive aussi qu'elles aient exclusivement trait à l'aide étrangère ou bien qu'elles fassent partie de déclarations générales sur le développement international, les relations extérieures ou la sécurité nationale.

\section{Un exemple : l'Allemagne}

Le gouvernement allemand a rendu public en 2001 son Programme d'action pour la lutte contre la pauureté à l'horizon 2015 qui présente les mesures concrètes que les différents ministères et organismes allemands prendront pour réduire de moitié d'ici à 2015 le nombre de personnes dans le monde vivant dans l'extrême pauvreté. Ce programme, élaboré avec la participation de la société civile et du secteur privé, fait du recul de la pauvreté l'une des priorités du gouvernement et l'objectif principal de la politique de développement de l'Allemagne. Il dresse une liste de dix domaines d'action prioritaires et de quelque 75 opérations spécifiques. Les dix domaines d'action prioritaires sont les suivants : i) stimuler l'économie et favoriser la participation active des populations pauvres ; ii) faire du droit à l'alimentation une réalité et mettre en œuvre des réformes agraires ; iii) ouvrir des perspectives commerciales aux pays en développement dans des conditions loyales ; iv) réduire la dette et financer le développement ; $v$ ) garantir l'existence de services sociaux de base et renforcer la protection sociale; vi) assurer l'accès aux ressources essentielles et favoriser la préservation de l'environnement; vii) respecter les droits de l'homme et les normes fondamentales du travail, viii) favoriser l'égalité entre les hommes et les femmes ; ix) asseoir les principes d'une bonne gouvernance ; $x$ ) éviter les conflits, favoriser la sécurité des hommes et encourager le désarmement.

\section{Les mécanismes ministériels}

L'environnement politique national explique pour une large part le nombre et la diversité des dispositifs ministériels mis en place pour la coopération pour le développement dans les pays membres du CAD. Objectivement, un système idéal est clairement placé sous la responsabilité d'une Autorité et dans la plupart des pays membres du CAD, l'organisme d'aide au développement relève d'un responsable politique identifiable. Il peut s'agir d'un ministre de la Coopération ou d'un ministre des Affaires étrangères dont les compétences dépassent les limites de la coopération pour le développement pour embrasser les affaires étrangères, voire le commerce international. Cependant, du fait du caractère complexe de la coopération pour le développement, il peut arriver que d'autres ministres soient chargés de certains aspects importants du programme d'aide de leur pays. Par exemple, les contributions aux institutions financières internationales comme la Banque mondiale peuvent être du ressort du ministre du Trésor ou du ministre de l'Économie. L'aide humanitaire peut être mise en œuvre indépendamment du reste du programme d'aide au développement et placée sous la responsabilité d'un autre ministre. Dans quelques pays membres du CAD, un seul ministre est responsable de l'ensemble du programme d'aide.

En ce qui concerne le rang du ministre chargé à titre principal de la coopération pour le développement, dans quelques pays membres du CAD seulement la coopération pour le 
développement relève d'un seul ministre, lequel siège au Conseil des ministres (premier cercle du pouvoir). Dans beaucoup d'autres cas, soit le ministre siège au conseil des ministres mais a un large éventail de responsabilités soit il est seulement chargé de la coopération pour le développement mais ne siège pas au Conseil des ministres. Aucune de ces situations n'est idéale du point de vue du développement. Dans le premier cas, le ministre peut défendre les questions de développement au plus haut niveau mais ne peut leur consacrer toute son attention et dans le deuxième cas, les questions de développement risquent de retenir peu l'attention au plus haut niveau du gouvernement ou bien d'être défendues par un ministre important qui par ailleurs ne s'occupe guère du programme de coopération pour le développement.

Dans les pays où plusieurs ministres s'occupent du programme de coopération pour le développement, il est important de mettre en place des mécanismes pour coordonner les activités et promouvoir les synergies. Dans certains cas, un mécanisme formel de coordination existe (comme en France, voir ci-après) sous la forme d'un comité. La composition, le calendrier des réunions et les missions de ce comité varient. Parmi les principaux facteurs qui semblent agir sur l'influence qu'il peut avoir, citons l'autorité dont il jouit, sa composition, la périodicité de ses sessions et des réunions de son secrétariat, son mandat et l'éventail des questions à traiter.

\section{Un exemple : la France}

Créé en 1998, le Comité interministériel de la coopération internationale et du développement (CICID) est chargé notamment de faciliter la coopération interministérielle. Il est présidé par le Premier ministre et compte parmi ses membres le ministre des Affaires étrangères, le ministre de l'Économie, des Finances et de l'Industrie, le ministre délégué à la Coopération, au Développement et à la Francophonie et d'autres ministres participant à la mise en œuvre du programme de coopération pour le développement de la France. Le CICID a pour mission de : i) définir les contours de la Zone de solidarité prioritaire (ZSP) regroupant les pays de concentration de la coopération française ; ii) fixer les orientations relatives aux objectifs et aux modalités de la politique de coopération internationale et d'aide au développement ; iii) veiller à la cohérence des priorités géographiques et sectorielles des diverses composantes de la coopération ; iv) assurer une mission permanente de suivi et d'évaluation de la conformité de l'aide aux objectifs fixés. Le CICID se réunit au moins une fois par an mais des réunions au niveau des hauts fonctionnaires peuvent être organisées. Le ministère des Affaires étrangères et le ministère de l'Économie, des Finances et de l'Industrie en assurent le co-secrétariat.

\section{Le contrôle parlementaire}

En tant que représentants élus des contribuables qui financent les programmes de coopération pour le développement, les parlementaires peuvent jouer un rôle important dans le suivi de la gestion et de la mise en œuvre de l'aide étrangère dans le cadre des sessions plénières du Parlement (par exemple pendant les séances de questions orales) ou lors des réunions ou des auditions des commissions parlementaires. Dans certains pays membres du CAD, comme au Royaume-Uni (voir ci-après), une commission ou une souscommission chargée exclusivement des problèmes de développement et des questions connexes a été créée. 
Un exemple : le Royaume-Uni

Depuis 1967, le développement international est examiné de près par des souscommissions ou des commissions du Parlement britannique qui sont aussi parfois plus généralement chargées de suivre les questions de politique étrangère. Parallèlement au Department for International Development (DFID), a été créée en 1997 une commission parlementaire du développement international chargée d'examiner les dépenses, la gestion et les stratégies du DFID. Depuis lors, cette commission, qui compte 11 membres, prépare des rapports et demande des données sur divers sujets, dernièrement par exemple: le contrôle des exportations stratégiques, les migrations et le développement; l'aide au développement dans les Territoires occupés. La commission peut interroger qui elle veut dans l'administration ou dans la société civile. Le gouvernement peut répondre aux rapports de la commission. Il existe aussi depuis longtemps au Parlement britannique un groupe informel appelé All-Party Parliamentary Group on Overseas Development, qui compte 20 membres et qui est chargé de suivre toutes les questions touchant le développement international, notamment dans ses aspects économiques, sociaux, politiques et humanitaires.

Dans certains pays membres du CAD, le Parlement est très actif et ministres et fonctionnaires consacrent beaucoup de temps à répondre aux demandes d'information des parlementaires. Dans ces pays, le rôle des parlementaires peut aller jusqu'à définir les grandes orientations et prendre les décisions importantes. Cependant, dans bien d'autres pays, le Parlement a une fonction plus modeste. L'existence d'une commission chargée de suivre les questions de développement ou un budget consolidé qui couvre la majorité des dépenses au titre de l'aide semblent être deux facteurs importants qui influent sur le degré d'intervention du Parlement dans les questions de coopération pour le développement.

\section{Le soutien de l'opinion publique à la coopération pour le développement}

Dans les pays membres du CAD, les citoyens sont d'importantes parties prenantes dans les programmes d'aide étrangère. Ils contribuent à ceux-ci en payant des impôts et en élisant les hommes politiques qui suivent, gèrent et mettent en œuvre les politiques d'aide ; ils bénéficient aussi de la prospérité et de la sécurité accrues qui résultent de la croissance économique et du recul de la pauvreté dans les pays en développement et les pays en transition. Dans le même temps, dans bon nombre de pays de l'OCDE, la compréhension des problèmes de développement est assez superficielle et l'appui en faveur de l'aide étrangère, bien que fort, est fondé sur l'hypothèse erronée que celle-ci sera consacrée à la résolution de crises humanitaires. L'effort d'aide des gouvernements est aussi en général considérablement surestimé 4 . Pour l'aide comme pour tout autre domaine d'intervention des pouvoirs publics, l'opinion publique a le droit d'être informée et de mieux comprendre les problèmes liés au développement international et à l'aide étrangère. Pour certains membres du CAD, cela est de la plus haute importance. En Suisse, par exemple, la loi fédérale sur la coopération au développement et l'aide humanitaire internationale contraint le gouvernement à assurer une éducation au développement à la population. D'autres gouvernements de pays membres du CAD considèrent qu'il n'est pas de leur ressort d'éduquer le public ou bien interdisent à leurs organismes d'aide au développement de le faire en adoptant des lois à cet effet. 


\section{L'éducation au développement}

Il est important de sensibiliser la population aux questions de développement et de faire mieux comprendre les problèmes qui se posent car, plus averti, le public est mieux à même de participer au débat sur la politique en matière de développement ; en outre, il est plus facile de mobiliser l'appui en faveur des programmes d'aide étrangère et de promouvoir réformes et améliorations. Dans les pays membres du CAD, l'opinion publique semble prédisposée à en apprendre davantage sur les questions de développement car son appui à l'aide aux pays en développement se maintient à un niveau élevé depuis près de 20 ans. Les personnes instruites, jeunes et vivant en milieu urbain sont généralement plus favorable à l'aide au développement ; cette conclusion pourrait être intéressante pour le ciblage des activités de sensibilisation ${ }^{5}$.

La réalisation des objectifs du millénaire pour le développement nécessitera une meilleure compréhension des questions de développement par l'opinion publique. Ces objectifs appellent en effet une augmentation des volumes de l'aide et une plus grande cohérence des politiques des pays donneurs, ce qui requiert une forte détermination politique et un soutien renouvelé de la part de l'opinion publique si des choix politiques difficiles favorisant le développement international doivent être faits. S'ils comprennent mieux les enjeux, les citoyens, et leurs représentants élus devraient être davantage convaincus que des choix politiques difficiles peuvent s'imposer mais que ceux-ci sont justifiés par les avantages à long terme qui en découlent.

L'un des aspects importants des travaux des organismes d'aide au développement est par conséquent d'informer le public des problèmes de développement en général et du programme national d'aide en particulier. Les activités d'information et d'éducation au développement des pays membres du CAD représentent généralement moins de $1 \%$ des dépenses d'APD ${ }^{6}$ mais depuis quelques années certains membres du CAD augmentent leur budget dans ce domaine. Cependant, pour la plupart des citoyens, les médias restent la principale source d'information sur les pays en développement et les problèmes de développement. Dans bon nombre de pays membres du CAD, les ONG peuvent aussi être une source importante d'information et être plus efficaces dans la sensibilisation aux problèmes de développement que les organismes d'aide ou tout autre organisme public.

Beaucoup de pays membres du CAD ont adopté une stratégie systématique et à long terme de la sensibilisation de l'opinion publique en s'aidant de diverses méthodes (voir ciaprès). Parmi celles-ci figurent la publication de rapports annuels, la mise à disposition de documents d'orientation importants, la coopération avec les écoles et les jeunes et l'intégration des problèmes de développement dans les programmes d'étude de certaines disciplines, la mise à disposition de matériels pédagogiques sur internet ${ }^{7}$, l'organisation de conférences nationales où la politique du gouvernement peut être présentée et débattue avec le public et la coopération avec des ONG et des institutions confessionnelles afin d'organiser des réunions d'information et des débats publics. Dans bien des cas, la communication porte sur les apports, par exemple les niveaux de l'aide et les allégements de dette plutôt que sur les résultats des efforts d'aide étrangère. Les organismes d'aide au développement pourraient accroître l'impact de leurs activités de sensibilisation en liant le développement international à des questions mieux connues du grand public comme la santé, l'environnement et la défense. Un autre moyen serait de centrer les efforts sur les résultats souhaitables du point de vue du développement en s'appuyant davantage sur les objectifs du millénaire pour le développement. 


\section{Un exemple : la Finlande}

Le ministère finlandais des Affaires étrangères a fait preuve de motivation et d'innovation dans son programme d'éducation au développement. Il travaille avec le Conseil national de l'éducation pour apporter son soutien à la mise en place de programmes d'initiation aux problèmes de développement dans les écoles. Les activités menées dans ce cadre englobent la mise au point de matériels interactifs utilisant le Web, des questionnaires-tests, des programmes de formation à l'intention des enseignants, des séminaires et des ateliers. Des campagnes nationales, comme celle de 2002 qui avait pour thème l'Afrique, donnent lieu à des festivals et des expositions qui attirent de très nombreux visiteurs. Le ministère a aussi financé une publication qui utilise des bandes dessinées pour présenter les problèmes de développement et qui s'intitule Comics with an Attitude: A Guide to the use of Comics in Development Information. Des ministres voyagent dans le pays pour informer les citoyens de l'action menée par la Finlande en matière de coopération pour le développement. Il a également été proposé d'utiliser les bureaux d'information de l'Union européenne pour diffuser des informations sur la coopération pour le développement.

\section{Suivre les tendances de l'opinion publique}

Les enquêtes d'opinion montrent que le soutien de l'opinion publique à l'aide au développement est élevé dans les pays membres de l'OCDE et est resté stable au fil du temps ; cette constatation est d'importante étant donné que la majorité des citoyens tend à penser que les niveaux d'aide sont supérieurs à ce qu'ils sont en fait. Le soutien des Européens en particulier se confirme de plus en plus. En 2002, la coopération pour le développement était " très " ou " plutôt » importante pour $86 \%$ d'entre eux, contre $76 \%$ en 1998. Par ailleurs, l'augmentation des dons aux organisations humanitaires et aux ONG s'occupant de développement témoigne également du soutien de l'opinion publique ${ }^{8}$.

Le soutien de l'opinion publique n'exclut pas les questions relatives au niveau des dépenses publiques. Par exemple, un sondage réalisé en 1995 aux États-Unis montre qu'à cette époque la majorité des personnes interrogées estimaient que le niveau des dépenses publiques en faveur de l'aide étrangère était trop élevé mais 80 \% d'entre elles approuvaient le principe selon lequel les États-Unis devaient aider les populations étrangères en réelle difficulté. En Italie, le fort soutien dont bénéficie l'aide au développement s'accompagne du souci que celle-ci soit utilisée de façon plus efficace. En outre, il peut aussi arriver que l'opinion publique soutienne fermement l'aide au développement sans bien comprendre les problèmes de développement et de pauvreté et la nature des programmes publics d'aide au développement.

Bon nombre d'organismes d'aide au développement prennent des mesures pour suivre l'évolution de l'opinion publique et utilisent à cette fin diverses méthodes. La Suède et la Norvège réalisent des sondages d'opinion annuels tandis que, pour la plupart des autres pays membres du CAD, les sondages sont trop coûteux et tendent donc à être moins fréquents même s'ils permettent d'obtenir tout un éventail d'informations précieuses. Des enquêtes plus ciblées ou portant sur un thème particulier peuvent être réalisées dans le cadre de sondages nationaux portant sur d'autres thèmes. Les enquêtes régulières de différents types sont intéressantes car elles peuvent permettre de suivre les tendances de l'opinion publique dans le temps et d'établir des liens entre ce que pensent les citoyens et l'évolution de la politique publique. Un petit nombre de membres du CAD doivent encore 
réaliser des travaux de recherche ou des enquêtes pour déterminer le degré de sensibilisation de l'opinion publique aux questions de développement et la mesure dans laquelle les problèmes sont compris tandis que d'autres n'ont pas même mis en place des dispositifs qui permettraient de réaliser régulièrement des études.

Récemment, les membres du CAD, la Commission économique des Nations unies pour l'Europe et l'OCDE ont travaillé ensemble pour améliorer la fiabilité et la comparabilité des données collectées dans le cadre des enquêtes d'opinion afin de faciliter une analyse plus approfondie des positions et tendances de l'opinion publique dans l'ensemble des pays membres du CAD. Il s'agit notamment d'élaborer un ensemble de questions communes qui pourrait être inclus dans des enquêtes nationales consacrées à la coopération pour le développement.

\section{Notes}

1. Lancaster, C. (1999), Aid to Africa: So much to do, so little done, University of Chicago Press, Chicago.

2. The Simons Report (1997), One Clear Objective: Poverty Reduction through Sustainable Development. Report of the Committee of Review, Canberra.

3. Voir : www1.oecd.org/media/release/dac_recommendation.pdf.

4. OCDE (2004), Mobiliser l'opinion publique contre la pauureté, Repères nº 2, OCDE, Paris.

5. OCDE (2003), L’opinion publique contre la pauvreté, OCDE, Paris.

6. Ibid.

7. Voir par exemple le site Internet d'AusAID www.ausaid.gov.au/globaled/default.cfm et le site du Global Education Centre de la Nouvelle-Zélande, www.globaled.nz, une ONG qui reçoit des fonds de NZAID.

8. OCDE (2003), L’opinion publique contre la pauureté, OCDE, Paris. 



\section{Chapitre 3}

\section{La politique de coopération pour le développement des pays membres du CAD à l'aube du $\mathrm{XXI}^{\mathrm{e}}$ siècle}

Les programmes de coopération pour le développement des pays membres du CAD diffèrent les uns des autres à de nombreux égards, et notamment par l'envergure, les types d'instruments utilisés, le nombre d'activités bénéficiant d'un soutien, l'origine des fonds, l'affectation des sommes allouées, les principaux pays partenaires et l'orientation sectorielle. Ces différences trouvent leur origine dans divers facteurs au nombre desquels les choix stratégiques et politiques des pays donneurs, les avantages comparatifs propres à chacun, des considérations historiques, culturelles, géopolitiques, stratégiques et l'intérêt porté par chacun à la question du développement. La diversité observée dans les programmes en faveur du développement a des répercussions essentiellement sur les pays partenaires dans lesquels la politique de développement et les pratiques des pays donneurs influent sur la faculté de faire de l'appropriation à l'échelon local et de l'instauration de véritables partenariats une réalité. Le fait de mettre l'accent sur la coordination entre les donneurs, ainsi que sur l'harmonisation et l'alignement de l'aide (voir chapitre 8), deurait favoriser l'amélioration de l'efficacité de l'aide et l'abaissement des coûts de transaction supportés par les pays partenaires. 


\section{Aide aux pays en développement et en transition}

En 2003, les pays membres du CAD ont consacré quelque 69.0 milliards USD d'APD nette aux pays en développement, soit l'équivalent de 0.25 pour cent de leur RNB cumulé (voir graphique 3.1). L'APD se compose de dons et de prêts assortis de conditions libérales versés essentiellement dans le but de favoriser le développement et l'amélioration du bien être des populations des pays bénéficiaires par des organismes du secteur public d'un pays donneur à un pays en développement ou à une organisation multilatérale active dans le domaine du développement. En 2002, le montant des dettes effacées représentait en effet 11 pour cent de l'APD contre 7 pour cent en 2001 et 6 pour cent en 2000. D'ici 2006, le montant de l'APD pourrait atteindre près de 88 milliards USD (aux prix et taux de change de 2003) si les engagements pris à l'occasion ou au moment de la Conférence internationale des Nations unies sur le financement du développement qui s'est tenue à Monterrey en 2002 sont honorés ${ }^{1}$.

Les apports destinés aux pays en transition ou à des organismes dont l'activité bénéficie essentiellement à des pays en transition sont considérés comme relevant de l'aide publique ${ }^{2}$. En 2003, les pays membres du CAD ont versé aux pays en transition plus de 7 milliards USD au titre de l'aide publique nette, les principaux donneurs étant la France (2.0 milliard USD), les États-Unis (1.5 milliards USD) et l'Allemagne (1.2 milliard USD).

Plus des deux tiers du montant total de l'aide extérieure distribuée par les membres du CAD ont été constitués d'apports bilatéraux, essentiellement sous forme de dons, dont les principaux bénéficiaires ont été la Chine, l'Inde et la République du Congo (voir graphique 3.2). L'aide accordée aux grands pays peut avoir un effet de levier puissant grâce aux transferts d'idées et de bonnes pratiques auxquels elle donne lieu même si elle est modeste, en volume, par rapport à la taille de l'économie des bénéficiaires. Et comme l'a démontré la crise qui a frappé l'Asie du Sud-Est en 1997, les avancées obtenues grâce à cette aide sur le front du développement risquent de ne pas être durables si les systèmes financiers et les systèmes de gestion des pays bénéficiaires sont de piètre qualité. Dans ces conditions, une assistance prolongée sera peut-être nécessaire pour faciliter la pérennisation des progrès accomplis. Dans les pays les plus pauvres en revanche, l'APD demeure une source de financement importante pour le secteur public car ces pays n'ont tout simplement pas encore la possibilité de se procurer auprès d'autres sources des sommes suffisantes pour financer leur développement : l'assiette des recettes publiques y est en effet encore trop faible et les financements privés trop rares, voire inexistants. Les donneurs procurent 40 pour cent, voire plus, des ressources publiques d'une bonne trentaine de pays en développement, au nombre desquels la Bolivie, Madagascar, le Népal et la Tanzanie ${ }^{3}$. Les chances des pays les moins avancés de devenir plus prospères dépendront dans une large mesure du maintien du niveau de l'APD sachant que ces pays devront de leur côté renforcer leurs capacités de générer et de mobiliser des ressources sur le plan intérieur tout en promouvant l'investissement privé. 


\section{Graphique 3.1. Aide publique au développement nette en 2003}
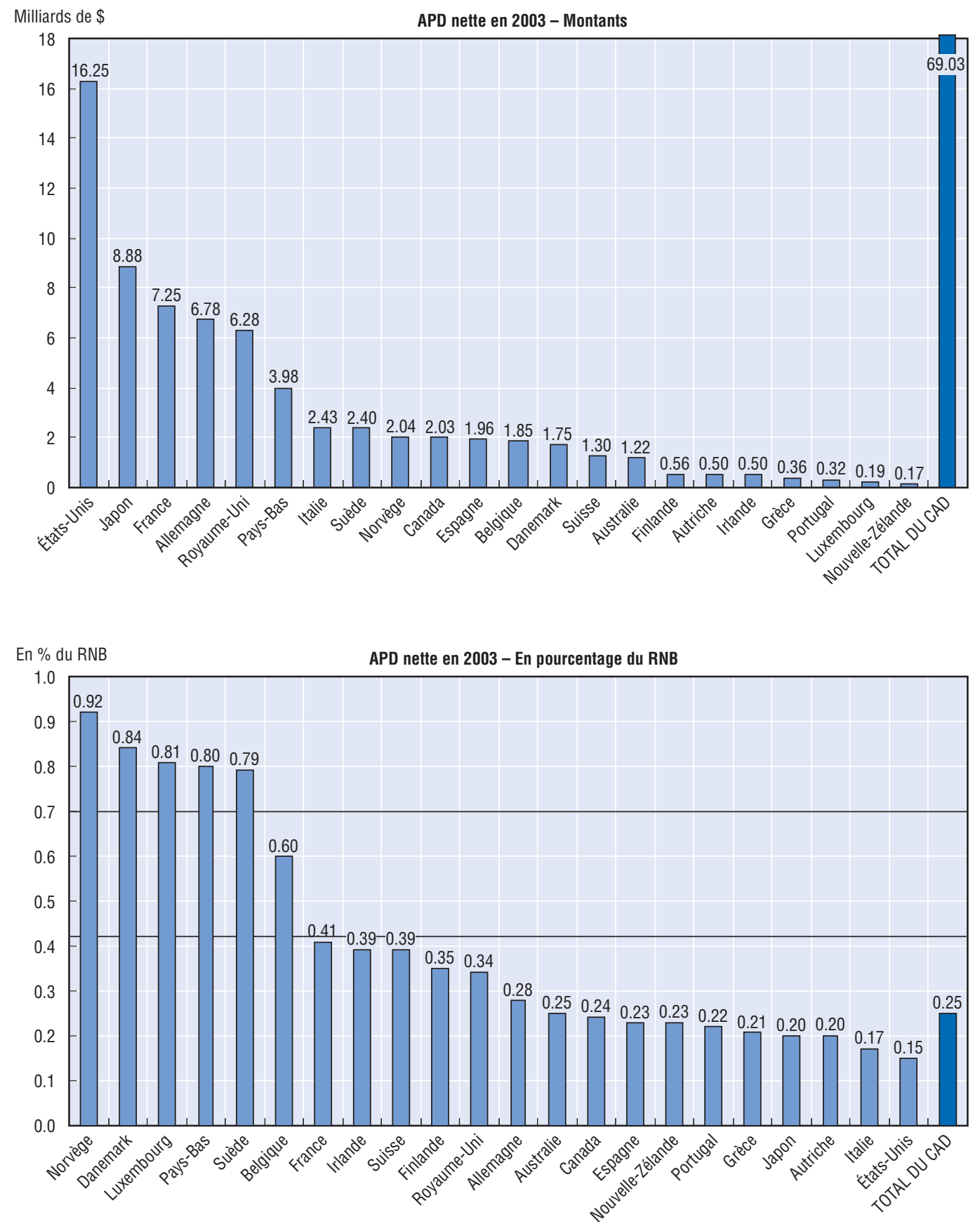

\section{Quelques caractéristiques des programmes d'aide extérieure}

Lorsqu'on les considère séparément, on observe une diversité remarquable dans les programmes de coopération pour le développement mis en œuvre par les pays membres du CAD, diversité qui s'explique dans une certaine mesure par des différences tant dans les choix politiques et stratégiques propres à chaque pays que dans leurs avantages comparatifs respectifs (se reporter à l'annexe A.1 pour avoir un aperçu du programme de chaque pays membre). Le nombre et le choix des principaux pays partenaires varient selon 


\section{Graphique 3.2. Vue d'ensemble de l'aide extérieure distribuée par les membres du CAD}

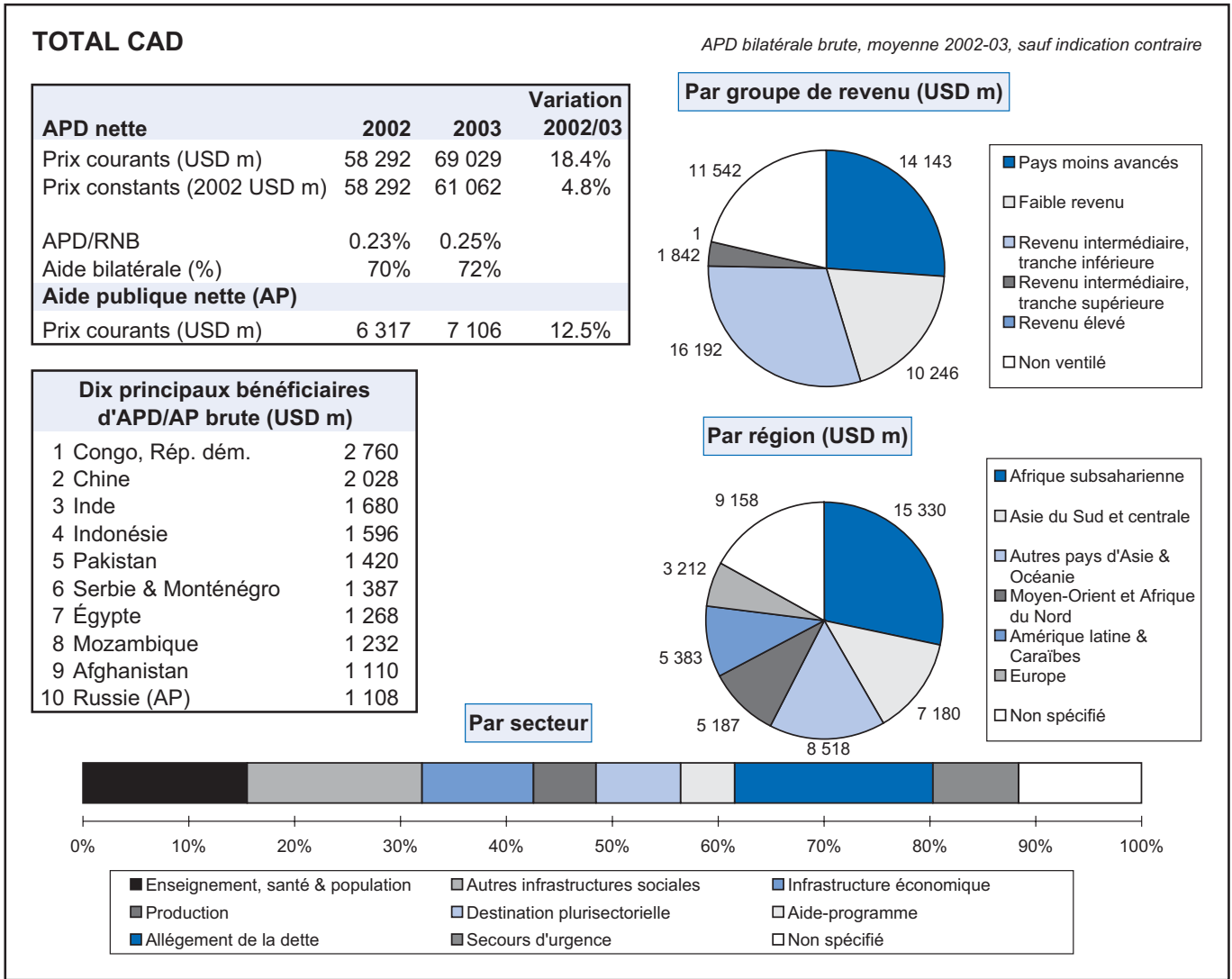

Source : Voir www.oecd.org/dac/stats.

les pays au gré de considérations historiques, culturelles, géopolitiques, stratégiques et aussi en fonction de l'intérêt porté par chacun à la question du développement. Certains membres du CAD ont à leur disposition une large palette d'instruments tandis que certains se refusent à accorder des prêts et que d'autres optent pour le soutien budgétaire ou l'envoi de bénévoles sur place, pour ne citer que ces trois possibilités. La part de l'APD multilatérale varie d'environ un quart à près des trois quarts du montant total de l'APD nette. Rares sont les pays membres du CAD qui sont également membres de toutes les banques régionales de développement et nombreux sont ceux qui sont devenus plus sélectifs dans le choix des organismes des Nations unies auxquels ils versent des contributions volontaires.

Pour acheminer l'aide dans les principaux pays qu'ils ont choisis pour partenaires, les pays membres du CAD ont recours à un large éventail d'instruments qui sont en principe sélectionnés et combinés en fonction d'une analyse de la situation sur place. Au Mozambique par exemple, les membres du CAD utilisent toute une panoplie d'instruments et la plupart d'entre eux jugent valable de maintenir des activités au niveau des provinces car celles-ci leur permettent d'évaluer l'impact des mesures prises par l'administration mozambicaine et de mettre à profit les enseignements tirés de l'expérience dans le cadre du dialogue sur les politiques à suivre qui se poursuit à l'échelon national. Les Lignes 
directrices du CAD : La réduction de la pauvreté décrivent de façon détaillée les principaux instruments de coopération technique et financière utilisés, et notamment les plus courants d'entre eux, à savoir l'aide-projet, l'aide sectorielle et l'aide-programme :

- L'aide-projet a généralement pour objet de dégager d'une réalité complexe un ensemble gérable de problèmes et de déterminer les apports nécessaires pour stimuler le développement local. En théorie, pour qu'ils concourent plus efficacement à la réduction de la pauvreté, les projets doivent être replacés dans le contexte plus vaste de la stratégie globale de développement, apporter des réponses aux multiples préoccupations des pauvres et viser à renforcer la capacité des populations pauvres d'assurer durablement leur subsistance 4 .

- L'aide sectorielle ou les approches sectorielles tendent vers l'adoption, sous la houlette de l'administration du pays partenaire, d'une politique et d'un programme de dépenses uniques au niveau sectoriel et doivent, dans la mesure du possible, reposer sur l'application de méthodes de gestion et de comptabilisation communes. Les programmes sectoriels exigent que l'on envisage différemment la gestion de l'aide et que l'on fasse preuve de davantage de modestie, que l'on accepte que le changement prenne du temps et que l'on s'appuie sur des relations de partenariat.

- L'aide-programme consiste à accorder des concours financiers, non liés à des activités précises menées dans le cadre d'un projet, à un pays partenaire afin d'appuyer son développement en général ; elle peut notamment prendre la forme d'un soutien à la balance des paiements ou d'un soutien budgétaire. Elle est souvent associée à des actions destinées à encourager les réformes au niveau macro-économique et/ou dans des secteurs définis.

L'ampleur des programmes bilatéraux d'aide extérieure "gérés " sur une base annuelle par les pays membres du CAD présente elle aussi des disparités notables. La notion "d'aide extérieure bilatérale gérée " donne une idée des sommes effectivement gérées par les organismes d'aide au développement des pays membres du CAD. Elle recouvre le montant total de l'APD bilatérale brute et le montant total de l'aide publique bilatérale brute dont sont soustraits les remises de dettes, le coût imputé des étudiants, l'aide aux réfugiés dans les pays donneurs et les dons au titre de l'aide publique à des territoires d'outre-mer. En 2002, les sommes correspondantes s'échelonnaient entre moins de 100 millions USD et plus de 13 milliards USD selon les pays, ceux-ci pouvant être regroupés en quatre grandes catégories :

- Sept donneurs gérant des programmes de faible envergure (des programmes d'aide extérieure bilatérale représentant moins de 300 millions USD) : l'Autriche, la Finlande, la Grèce, l'Irlande, le Luxembourg, la Nouvelle-Zélande et le Portugal.

- Neuf donneurs gérant des programmes d'envergure moyenne (des programmes d'aide extérieure bilatérale d'un montant compris entre 500 millions USD et 1500 millions USD) : l'Australie, la Belgique, le Canada, le Danemark, l'Espagne, l'Italie, la Norvège, la Suède et la Suisse.

- Quatre donneurs gérant des programmes de grande envergure (des programmes d'aide extérieure bilatérale d'un montant compris entre 2 milliards USD et 4 milliards USD) : l'Allemagne, la France, les Pays-Bas et le Royaume-Uni.

- Deux donneurs gérant des programmes à très grande échelle (des programmes d'aide extérieure bilatérale représentant plus de 9 milliards USD) : les États-Unis et le Japon. 


\section{Impact global des programmes d'aide extérieure}

Lorsqu'on les considère globalement, on est frappé par la fragmentation marquée des programmes d'aide extérieure mis en œuvre par les pays membres du CAD. Ces derniers font en effet transiter l'aide qu'ils distribuent à 186 pays en développement ou en transition au total par un grand nombre d'organismes publics, plus de huit pour certains d'entre eux. Les activités qu'ils financent sont menées par une multitude d'intermédiaires parmi lesquels des institutions du pays partenaire présentes à l'échelon national, provincial ou local, des organismes multilatéraux, des fonds mondiaux, des ONG et d'autres organisations de la société civile, des sous-traitants du secteur privé, des consultants et des bénévoles. L'aide peut prendre des forme diverses: actions de formation, conseils techniques, bourses accordées pour des études supérieures, fourniture de marchandises, notamment de médicaments et de denrées alimentaires, construction, entretien et réparation d'infrastructures, versements directs au profit des états bénéficiaires ou remises de dettes. Pendant la période comprise entre 1999 et 2001, les membres du CAD ont déclaré, dans le cadre du système de notification des pays créanciers, avoir entrepris quelque 35225 activités nouvelles par an dans le domaine du développement ${ }^{5}$, le coût moyen de chaque activité ressortant à 1.5 million USD.

Parce que les membres du CAD apportent leur soutien à un très grand nombre d'activités, les coûts de transaction supportés par les pays partenaires comme par les donneurs eux-mêmes sont élevés, ce qui réduit d'autant l'impact global de l'aide extérieure. En 2000, un pays en développement recevait en règle générale de l'aide d'une quinzaine de donneurs bilatéraux (et d'une dizaine d'organismes multilatéraux) ${ }^{6}$ et, pour l'ensemble des donneurs, la question qui se pose est de savoir comment contribuer à une meilleure adéquation de l'action de donneurs aux besoins des pays partenaires mettant au mieux à profit les avantages comparatifs de chacun. Les États membres de l'Union européenne sont tenus depuis 1992 par une convention de coordonner leurs politiques de coopération pour le développement et de se concerter sur leurs programmes d'aide ${ }^{7}$. Les efforts déployés jusqu'ici n'ont toutefois pas permis de ramener le nombre d'activités financées par l'aide à des niveaux plus raisonnables. Le consensus sur les grands objectifs et les grands axes de la coopération pour le développement devrait permettre d'asseoir solidement une collaboration plus étroite entre les donneurs, et notamment d'élargir les possibilités qui leur sont offertes de déployer des activités ou d'acheminer des fonds par l'intermédiaire d'autres donneurs. Quant à la recherche de moyens nouveaux et plus efficaces d'acheminer l'aide extérieure, elle devient une préoccupation de plus en plus urgente au fur et à mesure de l'entrée en scène de nouveaux donneurs (voir encadré 3.1).

\section{Mécanismes de coordination entre les donneurs}

Les pays membres du CAD considèrent que la coordination est un aspect fondamental, et même capital, et estiment qu'assurer une bonne coordination entre les donneurs est un moyen de contribuer à améliorer l'efficacité de l'aide extérieure et de réduire les coûts de transaction imposés aux pays partenaires. C'est pour cette raison qu'en dehors des initiatives axées sur l'harmonisation entre donneurs, on dénombre quelques dispositifs formels importants de dialogue entre les donneurs dont la mise en place n'est d'ailleurs pas récente. Il existe en outre sur le terrain un certain nombre de mécanismes de coordination mis à la disposition des donneurs et des pays partenaires dont l'importance ne cesse de grandir. 


\section{Encadré 3.1. Donneurs non membres du CAD}

Lorsqu'il a été créé en 1960, le CAD ne comptait que dix membres, chiffre qui a plus que doublé en une quarantaine d'années. La Grèce est le dernier pays à avoir intégré les rangs du CAD puisque son adhésion date de 1999. Certains autres pays membres de l'OCDE nourrissent le projet de faire partie du CAD dans les années à venir. Totalisant une APD nette de 279 millions USD en volume en 2002, la Corée peut d'ores et déjà s'enorgueillir de mener un programme d'aide d'envergure même si son ratio APD/RNB qui ressort à 0.06 pour cent demeure faible par comparaison avec la norme en vigueur parmi les membres du CAD. Certains pays et certaines économies non membres de l'OCDE, tels la Chine, le Taipei chinois, le Koweït, l'Arabie Saoudite et les Émirats arabes unis, sont également actifs, et se comportent même comme des acteurs importants, dans le domaine de la coopération pour le développement.

Depuis leur adhésion à l'Union européenne le $1^{\mathrm{er}}$ mai 2004, dix États d'Europe centrale et de la région méditerranéenne ont eux aussi acquis une importance nouvelle en tant que donneurs. Ils participent désormais au financement et à la gestion des programmes d'aide de la Communauté européenne. Non contents de l'augmentation sensible, liée à leur nouveau statut, de l'aide multilatérale qu'ils distribuent, certains de ces pays s'efforcent de renforcer leurs capacités institutionnelles de gérer et d'exécuter des programmes bilatéraux plus efficaces et plus étoffés. La République tchèque par exemple a affecté en 2002 une enveloppe de 45 millions USD à l'APD et de 9 millions USD à l'AP. Ces dernières années, elle s'est employée à renforcer, au sein de son ministère des Affaires étrangères, les capacités de coordonner les actions des 11 ministères qui concourent à la mise en œuvre de l'aide bilatérale, et elle a en outre mis en place un organe centralisateur de taille encore modeste qui pourrait préfigurer un organisme d'exécution susceptible d'être investi par la suite d'une large palette de responsabilités.

Très rares sont les pays qui ont effectivement les moyens de mettre en œuvre un programme de coopération pour le développement pertinent et dense dans tous les pays en développement du monde. La plupart des donneurs doivent dans une certaine mesure se montrer sélectifs dans le choix de leurs principaux partenaires, des secteurs visés ou des instruments utilisés pour acheminer l'aide s'ils veulent que le volume de leurs activités atteigne la masse critique requise pour que leurs efforts soient productifs et qu'ils aient un impact durable. La contribution des donneurs qui commencent tout juste à s'engager sur le terrain de la coopération pour le développement représente une manne supplémentaire venant s'ajouter aux ressources mobilisées au service du développement à l'échelle mondiale et aboutit souvent à une reconnaissance immédiate, dès la première expérience, du rôle positif que peut jouer l'aide extérieure. La valeur de cette aide peut toutefois être amoindrie si ces donneurs décident de suivre l'exemple de nombreux donneurs bilatéraux qui ont d'ores et déjà choisi de concentrer leur effort sur quelques pays en développement " performants » bénéficiant d'un soutien solide, comme le Mozambique, dans lesquels ils mènent à bien des programmes bilatéraux dotés de quelques millions de dollars chacun. Les membres actuels du CAD peuvent prêter leur concours aux autres donneurs en les aidant à repérer où leur expérience et leurs compétences spécifiques peuvent être mises à profit avec le meilleur bénéfice. Ils peuvent également montrer la voie en proposant des méthodes probantes de mise en œuvre d'une politique bilatérale de coopération pour le développement, consistant par exemple à déléguer l'acheminement de l'aide au développement bilatérale à un autre donneur ou à agir dans le cadre de partenariats silencieux. L'équilibre qu'il convient de trouver entre aide multilatérale et aide bilatérale et les avantages que l'on peut attendre, tant sur le plan de l'efficacité que dans l'optique d'un allégement du fardeau pesant sur les pays partenaires, d'une solution consistant à faire transiter une plus grande part de l'aide par des organismes multilatéraux sont également des aspects qui méritent de retenir l'attention des donneurs, qu'ils soient ou non membres du CAD. Conscient de l'importance et du rôle grandissants des donneurs qui ne sont ni membres de l'OCDE, ni membres du CAD, le Comité d'aide au développement étudie actuellement diverses options destinées à faciliter la coopération informelle et à répondre à l'intérêt grandissant que suscitent ses travaux auxquels les pays sont de plus en plus nombreux à vouloir participer. 


\section{Le Comité d'aide au développement de l'OCDE}

Les grands donneurs bilatéraux œuvrent ensemble au sein du CAD à l'amélioration de l'efficacité de leurs efforts communs à l'appui du développement. C'est dans le cadre de ces travaux que le CAD assume une fonction importante de coordination des politiques de ses membres. Les activités menées par le CAD relèvent principalement de quatre grandes catégories :

- L'adoption d'orientations stratégiques faisant autorité (voir encadré 3.2 pour de plus de détails sur les documents de référence publiés par le CAD).

- La réalisation d'examens mutuels périodiques portant un regard critique sur les politiques et les programmes des membres dans le domaine de la coopération pour le développement.

- L'ouverture d'une tribune favorisant les échange de données d'expérience et l'avènement d'un consensus au niveau international sur les questions stratégiques et les problèmes de gestion intéressant les membres.

- La publication de statistiques et de rapports sur les flux d'aide et autres apports de ressources à destination des pays en développement et en transition.

\section{L'Union européenne}

Les États membres de l'Union européenne sont tenus, en vertu du traité de Maastricht de 1993, de coordonner leurs politiques en matière de coopération pour le développement et de se concerter sur leurs programmes d'aide. L'objectif des États membres de l'Union européenne est d'améliorer la coordination, la cohérence et la complémentarité de leurs programmes d'aide. Actuellement, 15 des 25 États membres de l'Union européenne sont également membres du CAD.

\section{Partenariat stratégique avec l'Afrique}

La Banque mondiale a institué le Partenariat stratégique avec l'Afrique (PSA) en 1987 et a ainsi formé l'un des plus importants organes de coordination de l'aide destinée à l'Afrique subsaharienne. Les priorités assignées au PSA ont évolué et, au delà de la question du financement, recouvrent désormais le contexte, en termes de développement, dans lequel s'inscrit la réforme économique grâce à la mise en place de groupes de travail qui réfléchissent sur des thèmes précis en relation avec cet aspect. Le PSA a également vocation à veiller à ce que l'aide distribuée appuie dans tous les cas les stratégies nationales de lutte contre la pauvreté pilotées par les pays eux-mêmes, à ce que les efforts s'intensifient en vue d'améliorer la coordination et de rationaliser les exigences imposées au niveau des pays, et à ce que les capacités des administrations des pays africains soient renforcées.

\section{Mécanismes de coordination au niveau des pays}

Ces dispositifs reposent parfois sur les groupes mis en place par les pays partenaires pour suivre la mise en œuvre et évaluer l'impact des stratégies de lutte contre la pauvreté, sur des groupes thématiques ou sectoriels de coordination entre les donneurs ou sur les tables rondes organisées par les Nations unies et les réunions officielles des groupes consultatifs de la Banque mondiale. 


\section{Réunion des groupes consultatifs de la Banque mondiale}

Les réunions des groupes consultatifs ont principalement pour objet de promouvoir le dialogue sur les politiques à suivre entre les donneurs et un pays partenaire en particulier, mais aussi de mobiliser des ressources au service de son développement. L'une des fonctions essentielles de ces réunions a été d'offrir aux donneurs l'occasion de s'engager à répondre aux besoins des pays concernés en matière de financement. Ces dernières années, les discussions ont été avant tout axées sur la planification sectorielle et les questions ayant trait à la gouvernance et au développement participatif. Les réunions des groupes consultatifs sont de plus en plus souvent organisées dans les pays concernés afin de favoriser une plus large participation des acteurs locaux (parlementaires, représentants de la société civile, d'ONG et du secteur privé).

\section{Encadré 3.2. Documents de référence publiés par le CAD}

OCDE (1992), Manuel de l'aide au développement : Principes du CAD pour une aide efficace.

OCDE (1992-1995), Lignes directrices sur l'aide et l'environnement, nos 1-9.

OCDE (1995), Orientations du CAD concernant l'aide des donneurs en faveur du développement des capacités dans le domaine de l'environnement.

OCDE (1995), Le développement participatif et la bonne gestion des affaires publiques.

OCDE (1995), Lignes directrices sur la coopération pour le développement, L'expansion $d u$ secteur privé.

OCDE (1998), Les conflits, la paix et la coopération pour le développement à l'aube du XXI siècle.

OCDE (1998), Compendium du CAD des notions et approches relatives à l'égalité homme-femme.

OCDE (1999), Lignes directrices du CAD pour l'égalité homme-femme et le renforcement du pouvoir des femmes dans le cadre de la coopération pour le développment.

OCDE (2000), Pratiques efficaces pour mener une évaluation conjointe associant plusieurs donneurs.

OCDE (2001), Recommandation du CAD sur le déliement de l'aide publique au développement aux pays les moins avancés.

OCDE (2001), Evaluation Feedback for Effective Learning and Accountability.

OCDE (2001), Les lignes directrices du CAD. Prévenir les conflits violents : quels moyens d'action?

OCDE (2001), Les lignes directrices du CAD. La réduction de la pauvreté.

OCDE (2001), Les lignes directrices du CAD. Stratégies de développement durable.

OCDE (2001), Les lignes directrices du CAD. Renforcer les capacités commerciales au service du développement.

OCDE (2001), Articles sur la prévention des conflits et la coopération pour le développement. Les Dossiers du CAD, volume 2, $\mathrm{n}^{\circ} 3$.

OCDE (2002), L'égalité homme-femme dans les politiques sectorielles.

OCDE (2003), Lignes directrices et ouvrages de référence du CAD. Harmoniser l'aide pour renforcer son efficacité.

OCDE/Organisation mondiale de la santé (2003), Lignes directrices et ouvrages de référence du CAD. Pauvreté et santé.

OCDE (n.d.), Gender Equality Tip Sheets. 


\section{Notes}

1. La majeure partie de l'accroissement de l'APD devrait être imputable à cinq pays membres du CAD, à savoir l'Allemagne, les États-Unis, la France, l'Italie et le Royaume-Uni. Pour de plus amples informations, veuillez consulter OCDE (2004), " Progrès accomplis vers les Objectifs du millénaire pour le développement », Les Dossiers du CAD, vol. 5, $n^{\circ}$ 1, OCDE, Paris, pp. 51-70.

2. Se reporter à l'annexe A.3 pour des informations plus détaillées sur les notions statistiques.

3. Banque mondiale (2003), Rapport sur le développement dans le monde 2004. Des services pour les pauvres, Banque mondiale, Washington DC, p. 203.

4. On peut trouver des informations sur la gestion des projets et des programmes à l'annexe A.5.

5. Les activités en faveur du développement peuvent prendre des formes très diverses allant de la mise en œuvre de projets ou de programmes à des transferts de fonds ou à des livraisons de marchandises en passant par des actions de formation ou des projets de recherche, des opérations d'allégement de la dette ou le versement de contributions à des ONG.

6. Acharya, A., Fuzzo de Lima, A. et M. Moore (2004), Aid proliferation: How responsible are the donors?, Institute of Development Studies Working Paper 214, Brighton.

7. Voir l'article 180 de la version consolidée du traité instituant la Communauté européenne. 


\section{Chapitre 4}

\section{Origine et affectation des fonds}

Pour de nombreux pays membres du CAD, la diversité des origines des fonds affectés au financement de leur programme de coopération pour le développement est un aspect important. Le fait qu'il n'existe pas de budget consolidé de la coopération pour le développement peut être source de difficultés pour le contrôle et la comptabilisation des dépenses liées au développement. Améliorer la prévisibilité des budgets alloués aux activités en faveur du développement pourrait également faciliter la gestion et la planification de ces activités. Les décisions relatives à l'affectation des fonds dédiés au développement sont certes conditionnées par de multiples facteurs, mais répondent de plus en plus au souci d'améliorer l'efficacité et l'impact des actions entreprises sur la réduction de la pauvreté. 


\section{Origine des fonds affectés à l'aide extérieure}

\section{Dotations}

Les pays membres du CAD financent leurs programmes d'aide extérieure principalement au moyen de dotations annuelles votées par le Parlement national sur la base de propositions émanant du gouvernement. Un budget voté a force de loi et sert quelquefois à définir les grandes lignes du programme d'aide extérieure. En principe, un tel budget est par nature suffisamment général pour laisser au gouvernement, au ministre responsable ou aux hauts fonctionnaires qui le gèrent une certaine latitude pour ajuster les dotations en fonction de l'évolution des circonstances ou d'événements imprévisibles, notamment lorsque survient une situation d'urgence ou une crise humanitaire. Dans certains pays où le Parlement jouit d'une assez grande indépendance vis-à-vis de l'exécutif, les représentants du Peuple donnent toutefois des indications plus précises sur la répartition géographique des ressources, le niveau de l'aide réservée à certains pays ou à certaines régions ou à certaines finalités. Un tel mode de fonctionnement risque d'amoindrir l'efficience et l'efficacité des programmes d'aide extérieure dans la mesure où il contraint les gestionnaires de l'aide à se concentrer en priorité sur l'adaptation des programmes en cours et la répartition des nouvelles ressources en fonction des divers critères fixés par le Parlement.

Les pays membres du CAD qui disposent d'un budget unique pour financer l'ensemble des activités relevant de l'APD et de l'aide publique sont peu nombreux. On peut trouver à cette situation plusieurs explications. Certaines formes d'aide n'exigent pas de transferts de ressources et ne doivent donc pas faire l'objet d'un abondement; c'est le cas notamment des annulations de créances non performantes ou, pour les États membres de l'Union européenne, de la part des versements effectués par la Commission qui sont directement financés sur ses ressources propres. Il arrive aussi que d'autres dépenses non comptabilisables dans l'APD ne puissent être effectuées, ni gérées par l'organisme d'aide au développement/le ministère des Affaires étrangères, qu'il s'agisse des frais de subsistance des réfugiés durant la première année qu'ils passent dans un pays donneur ou des coûts supplémentaires supportés par les forces armées lorsqu'elles mènent à bien des activités en rapport, d'une manière ou d'une autre, avec le développement. Enfin, les enveloppes allouées par l'État sont parfois complétées par des fonds provenant d'autres sources, de budgets d'instances infranationales par exemple. Les Pays-Bas sont à ce titre un exemple intéressant puisque leur système illustre comment tous les postes relevant de l'aide extérieure peuvent être intégrés dans un cadre budgétaire global (voir ci-après).

Même lorsque dans un pays membre du CAD, il existe un seul ministère ou un seul organisme d'aide au développement responsable de la gestion de la grande majorité des programmes d'aide extérieure, il arrive parfois que des dotations destinées à financer les dépenses réalisées à ce titre puissent être imputées à d'autres ministères. Les contributions au budget central des grandes organisations multilatérales en particulier sont souvent versées directement par le ministère compétent plutôt que par l'organisme d'aide au développement/le ministère des Affaires étrangères. Il se peut par exemple que 
ce soit le ministère des Finances qui verse les contributions destinées aux institutions financières internationales, et le ministère de la Santé celles allant à l'Organisation mondiale du Commerce (OMC) même si l'organisme d'aide au développement effectue des versements complémentaires au profit d'activités spécifiques. Dans les pays membres du CAD où plusieurs ministères participent à la mise en œuvre d'activités relevant de l'aide extérieure, il se peut même qu'il n'existe tout simplement pas de «budget de l'aide ». En pareil cas, chaque ministère finance les activités en relation avec l'aide au développement qui sont de son ressort sur ses dotations budgétaires ordinaires propres et ce sont les sommes correspondant à ces activités qu'il convient d'additionner en fin d'exercice pour déterminer le montant total des dépenses affectées à l'aide.

\section{Un exemple : les Pays-Bas}

Le Budget consolidé de la coopération internationale (HGIS) a été mis en place en 1995 par l'administration néerlandaise pour faire en sorte que l'ensemble des activités relevant de l'aide extérieure soient regroupées dans un cadre global de planification. Le HGIS se décompose en cinq rubriques correspondant aux cinq axes prioritaires de la politique étrangère des Pays-Bas que sont : l'ordre international ; la paix, la sécurité et la stabilité ; l'intégration européenne ; une réduction durable de la pauvreté ; et les relations bilatérales. Le tableau présenté ci-après indique le poids relatif des enveloppes affectées à ces différents domaines prioritaires et en particulier le pourcentage de l'APD que représente chacun d'entre eux. Le HGIS se révèle très utile pour avoir une vue d'ensemble des dépenses effectuées par les divers ministères intervenant dans le domaine de la coopération pour le développement (y compris de la part de l'APD ne correspondant pas à des dépenses réalisées dans des pays en développement, par exemple les opérations d'allégement de la dette ou les frais d'aide aux réfugiés supportés par le pays donneur). Il facilite en outre la distinction entre les activités coïncidant avec la définition de l'APD et celles qui n'entrent pas dans cette définition.

La diversité des sources de financement des activités relevant de l'aide extérieure a deux conséquences (fâcheuses). Elle complique la tâche des parlementaires qui assurent le suivi et l'orientation des programmes d'aide en raison du nombre de dotations qu'il va leur falloir examiner, dont certaines qui n'ont à première vue aucun lien avec le développement. Elle risque par ailleurs de compromettre la capacité des administrations nationales de gérer, et en conséquence de maîtriser directement, l'ensemble des dépenses devant être comptabilisées dans l'APD, d'où une certaine incertitude sur la position de chaque pays, sur le plan tant quantitatif que chronologique, par rapport aux objectifs fixés en matière d'APD, et notamment par rapport au ratio APD/RNB visé.

L'une des questions qui reviennent sans cesse à propos de la gestion de fonds alloués à l'aide extérieure est la suivante : comment concilier la nécessité de situer la coopération pour le développement dans une optique à long terme, exigeant une planification à horizon pluriannuel, avec la pratique courante qui consiste à fixer chaque année les dotations affectées à l'aide? Dans beaucoup de pays membres du CAD, les procédures administratives habituelles obligent à dépenser, ou du moins à engager, en vue d'un décaissement rapide, les fonds affectés au titre d'un exercice budgétaire pendant cet exercice. Les gestionnaires de l'aide sont en conséquence soumis, dans certains pays, à de très fortes pressions qui les incitent à engager et décaisser rapidement les fonds, ce qui 
Tableau 4.1. Budget consolidé de la coopération internationale (HGIS)

\begin{tabular}{|c|c|c|c|c|}
\hline & $\begin{array}{l}\text { Total HGIS } \\
\text { en } € 1000\end{array}$ & $\begin{array}{l}\text { Dont: APD } \\
\text { en } € 1000\end{array}$ & $\begin{array}{c}\text { Total HGIS } \\
\text { en } \%\end{array}$ & $\begin{array}{c}\text { Dont : APD } \\
\text { en } \%\end{array}$ \\
\hline 1. Ordre international & 142387 & 17951 & 3 & 0 \\
\hline 2. Paix, sécurité et stabilité & 477193 & 239155 & 9 & 5 \\
\hline \multicolumn{5}{|l|}{ Dont: } \\
\hline Aide humanitaire & 168586 & 164101 & 3 & 3 \\
\hline Bonne gouvernance, droits de l'homme et construction de la paix & 42027 & 11654 & 1 & 0 \\
\hline 3. Intégration européenne & 36440 & 0 & 1 & 0 \\
\hline 4. Réduction durable de la pauvreté & 3433365 & 3156838 & 65 & 60 \\
\hline A. Coopération bilatérale pour le développement & 1277001 & 1197462 & 24 & 23 \\
\hline B. Coopération multilatérale pour le développement & 1013234 & 874913 & 19 & 17 \\
\hline \multicolumn{5}{|l|}{ Dont: } \\
\hline Union européenne & 423440 & 299368 & 8 & 6 \\
\hline Nations unies & 305806 & 298533 & 6 & 6 \\
\hline Institutions financières internationales & 283988 & 277012 & 5 & 5 \\
\hline C. Coopération privée pour le développement & 1143130 & 1084463 & 22 & 21 \\
\hline \multicolumn{5}{|l|}{ Dont: } \\
\hline ONG & 745600 & 745146 & 14 & 14 \\
\hline Recherche et sensibilisation à l'échelon international & 137192 & 131317 & 3 & 2 \\
\hline Secteur privé & 260338 & 208000 & 5 & 4 \\
\hline 5. Relations bilatérales & 499231 & 200845 & 9 & 4 \\
\hline \multicolumn{5}{|l|}{ Dont: } \\
\hline Droit d'asile, migrations et services consulaires & 202447 & 194420 & 4 & 4 \\
\hline 6. Autres & 682268 & 196032 & 13 & 4 \\
\hline TOTAL & 5270884 & 3810821 & 100 & 72 \\
\hline
\end{tabular}

Source: Ministère des Affaires étrangères des Pays-Bas (2004).

tend à attirer indûment leur attention sur les apports financiers plutôt que sur les résultats escomptés ou les retombées réelles des activités menées en faveur du développement.

La question du décaissement des fonds n'est pas sans rapport avec leur affectation. Il est généralement plus facile de définir et d'autoriser que de mettre en œuvre des activités dans le domaine du développement : en effet, nombre de difficultés ayant pour effet de retarder considérablement, voire de rendre carrément impossible, l'exécution de certains projets peuvent surgir. Il arrive par exemple que les responsables de la mise en œuvre d'une activité éprouvent des difficultés à recruter du personnel, qu'il leur faille du temps pour obtenir les autorisations légales ou qu'ils aient à résoudre des problèmes techniques. Les donneurs sont aussi parfois amenés à prendre des décisions politiques en réaction à des évolutions majeures dans la situation des pays partenaires, et à choisir de reporter ou d'annuler des versements au titre du soutien budgétaire ou des contributions à des programmes sectoriels, voire à annuler l'ensemble des activités d'État à État, ce qui peut aboutir, dans certains cas, à une accumulation de crédits engagés, mais non dépensés. A moins que l'organisme d'aide au développement, ou que l'unité responsable des programmes menés dans tel ou tel domaine, ne dispose de la latitude voulue pour pouvoir redéployer les fonds vers d'autres activités ou pour procéder à un report en avant des crédits non dépensés (voir ci-après), la situation dans laquelle on se retrouve alors risque de légitimer des interrogations quant au bien-fondé d'un accroissement éventuel des enveloppes budgétaires. Une analyse des crédits non dépensés peut donc éventuellement aider les organismes d'aide au développement à mettre au jour les problèmes récurrents concourant à leur accumulation. 
Un exemple : la Nouvelle-Zélande

La Loi sur la responsabilité budgétaire adoptée par la Nouvelle-Zélande en 1994 oblige le gouvernement néo-zélandais à présenter des projections à horizon mobile sur trois ans pour chaque ligne budgétaire, notamment pour le budget de l'aide, ce qui permet de conférer un caractère plus prévisible aux dotations budgétaires au plus grand bénéfice de la continuité dans la planification et la gestion de l'aide. Par ailleurs, les responsables du programme néo-zélandais de coopération pour le développement peuvent effectuer des dépenses excédant l'enveloppe budgétaire annuelle qui leur est attribuée dans une proportion allant jusqu'à 10 pour cent ou reporter jusqu'à 20 pour cent de cette enveloppe sur l'exercice budgétaire suivant, ce qui réduit d'autant l'influence des facteurs incitant les gestionnaires de l'aide à dépenser les sommes qui leur sont allouées dans un délai imposé en fonction de critères extérieurs et indépendamment de l'évolution de la situation dans les pays en développement.

\section{Autres sources de financement}

En dehors des fonds distribués par des instances infranationales, les budgets affectés à l'aide qui sont votés par les Parlements nationaux peuvent être complétés de diverses manières. Les annulations de dettes par exemple représentent une part croissante de l'APD (voir ci-après). La Banque mondiale et les banques régionales de développement se procurent une proportion notable des ressources qu'elles redistribuent sous forme de prêts sur les marchés de capitaux internationaux et certains pays membres du CAD qui comptent des institutions bancaires au sein de leur système de coopération pour le développement, comme l'Allemagne, la France et le Japon, prêtent également aux pays en développement et en transition des capitaux levés sur les marchés internationaux. Les sommes encaissées au titre des remboursements du principal (amortissement du prêt) et du paiement des intérêts dus sur des prêts antérieurs constituent également une source de financement. En général, ces sommes sont restituées au Trésor ou réintégrées dans le budget de l'administration centrale même si dans un petit nombre de pays, elles peuvent être réaffectées au financement d'activités nouvelles en faveur du développement.

\section{Un exemple : l'Italie}

L'Italie a opté pour une conception souple de l'allégement de la dette. Elle va plus loin par exemple que l'initiative en faveur des PPTE en incluant les pays à revenu intermédiaire de la tranche inférieure dans ces accords d'allégement de dette. La législation italienne autorise non seulement l'annulation des créances relevant de l'APD, mais aussi l'effacement des dettes commerciales dans une proportion pouvant atteindre 100 pour cent. Les annulations de dettes sont conditionnées par le respect de certains critères par le pays bénéficiaires : bonne gouvernance, refus de la guerre et détermination solide à œuvrer à la lutte contre la pauvreté et au développement social et humain. L'Italie sait faire preuve de souplesse dans l'application des conditions qu'elle impose en matière de traçabilité des fonds et tenir compte du climat qui règne dans le pays partenaire et de la capacité de ce dernier à supporter le fardeau que représente l'application de mesures de contrôle strictes des transactions financières. Dans certains pays, les ressources dégagées par les opérations d'annulation de dettes sont affectées à un fonds local, géré conjointement par l'Italie et les autorités locales, celles-ci devant exercer un 
contrôle rigoureux des dépenses et garantir en même temps que les sommes concernées sont effectivement affectées au financement d'activités visant à faire reculer la pauvreté. Dans certains pays partenaires comme le Mozambique, où la confiance est de mise entre la communauté des donneurs et l'administration du pays, les annulations de dettes sont assorties d'un minimum d'exigences et ce, afin de ne pas imposer une charge administrative trop lourde au pays partenaire.

Les fonds provenant des prélèvements fiscaux sur les jeux de hasard et des biens saisis dans des affaires de trafic de stupéfiants (voir ci-après) constituent peut-être un moyen moins connu, mais néanmoins utilisé par certains pays membres du CAD pour financer des activités en faveur du développement.

\section{Un exemple : la Belgique}

Le Fonds belge de survie emploie les bénéfices nets générés par la loterie nationale belge pour financer des activités en faveur du développement dans quelques-uns des pays les plus pauvres de la planète. La création de ce fonds, baptisé initialement Fonds de survie pour le tiers monde, par une loi votée en 1983 constituait une réponse à l'émotion qu'avait suscitée à l'époque dans l'opinion publique l'annonce du nombre de victimes de la sécheresse en Éthiopie et dans d'autres zones de l'Afrique subsaharienne. Doté au départ de 248 millions EUR, le Fonds a bénéficié, en vertu d'une nouvelle loi promulguée en 1999, d'une dotation supplémentaire de 250 millions EUR, versée par tranches annuelles de 18.6 millions EUR minimum. Les programmes financés par le Fonds belge de survie privilégient une démarche globale et visent à améliorer la sécurité alimentaire et nutritionnelle des familles et des communautés locales dans les zones rurales et semi-urbaines. Les projets sont menés à bien conjointement avec des ONG ou des organismes multilatéraux (en particulier le FIDA, le PNUD, l'UNICEF et l'OMS). Entre 1984 et 2002, le fonds a financé environ 125 projets représentant au total 287 millions EUR.

\section{Un exemple : le Luxembourg}

La Convention des Nations unies contre le trafic illicite de stupéfiants et de substances psychotropes invite les signataires à reverser aux Nations unies les sommes provenant des biens saisis dans des affaires de trafic de stupéfiants - notamment les fonds saisis dans le cadre d'affaires de blanchiment de capitaux - afin de lui permettre de financer les actions qu'elle mène dans ce domaine. Sur les 168 parties à la convention, le Luxembourg est jusqu'ici le seul signataire à avoir répondu à cette invitation. Les sommes collectées par le Luxembourg sont d'abord versées au Fonds de lutte contre le trafic de stupéfiants. Leur affectation est ensuite décidée par une commission interministérielle composée de représentants du ministère des Finances (en sa qualité de président de la commission) et des ministères des Affaires, de la Santé et de la Justice. Depuis la création de ce fonds en 1993, les projets approuvés se chiffrent à plus de 11 millions EUR et s'adressent principalement à des pays jugés prioritaires dans le programme du Luxembourg en matière de coopération pour le développement ou par l'Office des Nations unies contre la drogue et le crime. Les activités financées par les Nations unies couvrent notamment des actions de formation à l'intention des responsables de la lutte contre la drogue en Argentine, en Bolivie, au Chili et au Pérou et des 
programmes de développement alternatifs destinés à éliminer la dépendance économique vis-à-vis de la production de substances illicites en Bolivie, au Laos et au Viêt-nam.

\section{Nouvelles sources de financement}

Ayant pris conscience du fait que pour atteindre les Objectifs du millénaire pour le développement, il faudra mobiliser au bénéfice de l'aide un surcroît considérable de ressources - d'après les estimations, le surplus nécessaire devrait représenter pas moins de 50 milliards USD par $a^{1}$ - les membres du CAD ont commencé à étudier les possibilités de financement du développement autres que l'APD telle qu'elle est pratiquée actuellement. Les principales options envisagées sont les suivantes :

- L'instauration de taxes prélevées à l'échelle mondiale sur les transactions monétaires, la consommation d'énergie ou les ventes de médicaments.

- La collecte de contributions volontaires du secteur privé que ce soit sous forme de donations, grâce à la création de loteries destinées à stimuler les dons du secteur privé au niveau mondial, grâce à l'émission d'obligations à primes à l'échelle mondiale ou à la création de fonds mondiaux.

- Les mesures d'ingénierie financière, recouvrant notamment des mécanismes de financement anticipé de l'aide grâce à la création d'une Facilité de financement internationale ${ }^{2}$, proposition qui consiste utiliser de manière ciblée les nouveaux droits de tirage spéciaux (DTS) émis par le FMI, et un renforcement des garanties publiques.

Chacune de ces options a des avantages et aussi des inconvénients tant sur le plan économique que sur le plan politique. Si elles sont relayées par une volonté politique renouvelée, un soutien accru de la part de l'opinion publique et une évolution des mentalités dans quelques-uns des pays membres du CAD, certaines d'entre elles pourraient se révéler prometteuses pour accroître les ressources allouées à l'aide. Les membres du CAD pourraient s'inspirer dans une large mesure de ces quelques propositions en les adaptant pour les appliquer unilatéralement. Il n'en demeure pas moins que la manière la plus simple d'accroître l'aide consiste à faire voter par les Parlements nationaux des dotations budgétaires supplémentaires.

\section{Affectation des fonds}

L'un des aspects les plus difficiles à cerner de la coopération pour le développement concerne la manière dont les décisions relatives à la répartition des fonds affectés à l'aide extérieure sont prises, que ces décisions portent sur la répartition entre le canal bilatéral et le canal multilatéral ou sur la ventilation entre les différents postes à l'intérieur de chacune de ces deux catégories. Dans la plupart des pays membres du CAD, il est rare que les schémas de répartition fassent l'objet d'un profond remodelage : en effet, les engagements établis de longue date sont généralement maintenus, les changements prenant le plus souvent la forme d'ajustements mineurs effectués à intervalles réguliers. Pourtant, nombre de pays membres du CAD procèdent à des évaluations plus critiques que par le passé de leurs engagements vis-à-vis de certaines organisations multilatérales et de leurs principaux partenaires et se montrent davantage disposés à se retirer d'organismes multilatéraux dont ils jugent les performances insuffisantes ou de programmes d'État à État mis en œuvre dans des pays peu respectueux des droits de l'homme ou des règles de 
la démocratie. La fin de la Guerre froide et le souci grandissant de veiller à l'efficacité de l'aide les incitent probablement à adopter une démarche plus stratégique et plus ciblée.

La part de l'aide fournie par les pays membres du CAD qui transite par le canal multilatéral représente généralement, à quelques notables exceptions près, entre un quart et un tiers du montant total de l'APD. Un petit nombre de pays ont pour principe de faire transiter une part donnée de leur aide par le canal multilatéral. L'aide multilatérale prend la forme de souscriptions au capital d'institutions multilatérales versées par l'État membre ou de contributions, pour des montants laissés à la discrétion de chaque pays, à des fonds gérés par des organismes multilatéraux, en particulier par des organismes des Nations unies. Les souscriptions au capital d'institutions multilatérales sont fixées à l'avance et ne donnent normalement pas lieu à d'autres décisions tant que l'adhésion à l'institution concernée n'est pas remise en question.

Les pays membres du CAD ont en revanche des décisions importantes à prendre concernant la répartition de leurs contributions volontaires versées à des institutions multilatérales et de leur aide bilatérale : ciblage géographique, nombre et choix des principaux pays partenaires et secteurs visés en priorité. Les directives qui doivent présider à ces choix sont parfois énoncées dans le texte législatif servant d'armature au programme d'aide ou dans une déclaration d'orientation de portée générale. Pour certains pays membres du CAD, le choix des principaux partenaires résulte de l'existence de liens historiques et politiques forts entre le pays donneurs et certains pays ou groupes de pays ou s'explique par la proximité géographique.

\section{Choix des pays partenaires et répartition des crédits}

Les pays membres du CAD financent des activités en faveur du développement dans un grand nombre de pays partenaires, souvent plus de 100, au moyen des diverses formules à leur disposition : co-financement d'ONG, aide humanitaire et projets axés sur des thèmes précis tels que l'égalité homme-femme, le VIH/sida, la démocratie et la bonne gestion des affaires publiques ou le développement du secteur privé. Dans certains cas, on observe un décalage sensible entre la liste des principaux bénéficiaires effectifs de l'aide extérieure distribuée par un pays membre du CAD et celle des pays désignés par lui comme ses principaux partenaires, et l'on s'aperçoit que les donneurs ne consacrent parfois qu'une faible part de leur aide bilatérale aux pays qu'ils ont choisis pour être leurs principaux partenaires.

Plusieurs pays membres du CAD ont décidé de s'employer à instaurer des partenariats plus solides avec un nombre plus restreint de pays partenaires pour leurs programmes d'État à État. Ce recentrage de leur effort d'aide est dans certains cas à replacer dans le contexte de l'avènement d'un nouveau programme en faveur du développement ou d'un nouveau mécanisme de financement, d'une politique de décentralisation accrue se traduisant par des transferts de responsabilités aux bureaux sur place ou d'une volonté de privilégier un ciblage resserré répondant au souci d'améliorer l'efficacité de l'aide. La perspective d'un accroissement, ou d'une contraction, notable de l'aide peut susciter une réflexion, au sein même des organismes d'aide au développement, sur les raisons pour lesquelles un programme d'aide extérieure est conçu de telle ou telle manière, et ce sont les conclusions de cette réflexion qui pourront éclairer les décision futures concernant notamment le choix des principaux partenaires et le montant des budgets requis. 
Limiter le nombre de pays dans lesquels les organismes d'aide sont actifs représente un véritable défi et la décision de recentrer l'action sur un nombre plus restreint de pays partenaires ne conduit que rarement un pays donneur à cesser rapidement et totalement toute activité dans un pays bénéficiaire. En règle générale, seules les activités d’État à État sont touchées et les activités en cours sont parfois poursuivies jusqu'à leur terme. Le Danemark par exemple a décidé récemment, compte tenu de la rapidité de l'évolution économique, politique et sociale que connaît le pays, d'interrompre progressivement son programme de coopération avec le Bhoutan, la cessation des activités devant s'étaler sur dix ans. Parallèlement, le Danemark met en place un nouveau programme dans le secteur de l'éducation au Bhoutan ${ }^{3}$.

Pour aider les donneurs à améliorer l'efficacité de leur aide, l'OCDE a organisé en 2003 un séminaire d'experts ${ }^{4}$ consacré au thème de la sélection des pays bénéficiaires et de la détermination des enveloppes budgétaires, et elle a rendu publiques des informations sur la manière dont les Pays-Bas et les États-Unis abordent cet aspect de la gestion de l'aide (voir encadré 4.1). Les participants sont convenus que tout ce qui a trait à la sélection des pays bénéficiaires relève en dernier ressort de préférences éminemment politiques, mais que le recours à des modèles d'analyse de qualité permettrait d'apporter un éclairage utile sur le fonctionnement des mécanismes de décision à l'œuvre qui ne vont toutefois pas sans risques. D'un côté, si les analyses qui les sous-tendent pèchent par excès de complexité, les modèles d'affectation de l'aide risquent de rester lettre morte. D'un autre côté, si les analyses sont trop simplistes et font abstraction de variables importantes, les résultats risquent de perdre de leur pertinence et d'être dans ce cas aussi passés sous silence. Il convient donc de trouver un juste équilibre afin de ne tomber ni dans l'un, ni dans l'autre de ces pièges. Une autre question qui se pose à l'ensemble de la communauté des donneurs a été soulevée durant le séminaire : si tous les donneurs utilisent les mêmes critères pour déterminer la répartition de l'aide entre les pays bénéficiaires, on risque d'aboutir à une situation où l'aide extérieure va toujours aux mêmes pays partenaires (ceux qui sont jugés "performants"), ce qui pourrait entraîner une diminution du rendement de l'aide, lequel pourrait même devenir négatif, dans les pays sélectionnés, ainsi d'ailleurs qu'une pénurie de financement et une menace d'aggravation de la pauvreté et d'instabilité dans les autres pays. Les participants sont parvenus à la conclusion que chaque donneur devrait s'intéresser à ce que font les autres donneurs dans les pays qui sont ses principaux partenaires ou dans ceux qui pourraient devenir pour lui de nouveaux partenaires. Il conviendrait d'analyser sous forme de tableaux les besoins en matière d'aide et les capacités des donneurs aux niveaux sectoriel, national et international. La coordination de l'effort d'aide, entre pays et à l'intérieur de chaque pays, est une nécessité absolue.

\section{Ciblage sectoriel}

La sélection des secteurs dans lesquels les pays membres du CAD sont actifs sur le terrain dans les pays dont ils ont fait leurs principaux partenaires demeure un aspect essentiel de l'élaboration d'un programme bilatéral d'État à État. Plusieurs pays membres du CAD restreignent, ou s'efforcent de plus en plus de restreindre, le nombre de secteurs dans lesquels ils déploient des activités dans chacun de leurs principaux pays partenaires. Le Danemark, par exemple, se donne pour objectif de travailler dans quatre secteurs au maximum dans chacun de ses principaux pays partenaires, et la Finlande et les Pays-Bas 


\section{Encadré 4.1. Séminaire d'experts organisé par l'OCDE sur l'efficacité et la sélectivité de l'aide : intégrer de multiples objectifs dans le processus de répartition de l'aide}

La conclusion la plus importante de ce séminaire, auquel ont pris part des représentants d'organismes d'aide au développement, d'ONG et d'instituts de recherche de pays de l'OCDE et de pays en développement est la suivante : on observe une convergence de vues nette et même de plus en plus marquée sur la manière de procéder pour faire en sorte que la répartition de l'aide entre les pays bénéficiaires serve plus efficacement l'objectif de réduction de la pauvreté. Les principaux critères à prendre en compte sont :

- Le niveau de pauvreté (représenté par le revenu moyen par habitant), l'objectif étant de diriger une large part de l'APD vers les pays à faible revenu.

- L'incidence de la pauvreté (représentée par le chiffre de la population).

- Le performances des pays partenaires sur le front du développement.

Des divergences notables subsistent toutefois à la marge concernant par exemple la pondération en fonction respectivement de la population et des performances en termes de développement, ainsi que les méthodes de mesure de ces performances.

Un certain nombre de pays membres du CAD, parmi lesquels les Pays-Bas et les États-Unis, s'appuient sur ces données pour orienter leurs décisions concernant la répartition par pays. Leur intention en agissant ainsi n'est pas d'ôter aux responsables politiques les décisions finales, mais de les informer sur ce qui ressort de l'application de ces critères et pondérations. Autrement dit, ce sont les résultats de cette approche quantitative, plutôt que des préférences fondées sur les tendances de l'année précédente ou sur toute autre considération ponctuelle, qui motivent les décisions prises en matière d'affectation de l'aide.

Aux Pays-Bas, la sélection des principaux pays partenaires pour les programmes d'État à État et la détermination du montant de l'aide consentie à chaque pays résultent certes de processus éminemment politiques auxquels sont associés les ministres du gouvernement et le Parlement, mais il est manifeste depuis quelques années que les données tirées d'études portant sur l'efficacité de l'aide sont devenues un aspect important des choix qui sont opérés. Les Pays-Bas appliquent un certain nombre de critères sur lesquels sont fondées leurs évaluations :

- Pour la sélection des pays effectuée au second semestre 2003, la procédure a été modifiée. Le choix des pays partenaires tient compte non seulement de la situation telle qu'elle est, mais aussi des perspectives à long terme. Les principaux critères retenus demeurent la pauvreté (l'accès aux concours de l'IDA), la bonne gestion des affaires publiques et la politique sociale. Les autres critères utilisés sont les besoins en matière d'aide, la valeur ajoutée générée par les activités de coopération pour le développement menées par les Pays-Bas, ainsi que des considérations de politique étrangère. La nouvelle procédure a permis de dresser une liste de 36 pays partenaires (dont l'Afghanistan) avec lesquels les Pays-Bas ont résolu de nouer des relations de coopération au service du développement s'inscrivant dans le long terme.

- La répartition de l'enveloppe entre les pays de la liste tient compte à la fois des performance et des besoins. Les indicateurs de performance ont trait aux politiques économique et sociale, à la gouvernance (Évaluation de la performance de la politique et des institutions des pays effectuée par la Banque mondiale) et au respect des droits de l'homme, et tiennent compte en outre des résultats de l'évaluation du programme d'aide en cours d'exécution dans les pays. Les besoins sont appréciés en fonction de trois indicateurs par tête : le PNB, l'APD et la dette. Ces chiffres sont additionnés, et ensuite 


\section{Encadré 4.1. Séminaire d'experts organisé par l'OCDE sur l'efficacité et la sélectivité de l'aide : intégrer de multiples objectifs dans le processus de répartition de l'aide (suite)}

majorés de $50 \%$ dans le cas des pays les moins avancés et des pays d'Afrique subsaharienne. Le résultat final est ensuite appliqué à l'enveloppe totale, ce qui donne une dotation par tête qui est alors multipliée par la racine carrée de la population $d u$ pays afin d'introduire un biais en faveur des petits pays. Enfin, un seuil de 10 millions EUR a été prévu pour éviter d'avoir à conserver des programmes de trop faible envergure, et de ce fait irréalisables. Fin 2004 cependant, une révision du système a été entreprise.

Les États-Unis appliquent pour leur part un ensemble de critères concrets, objectifs et précis pour choisir les pays qui pourront prétendre bénéficier de financements au titre du nouveau Millennium Challenge Account. Durant les deux premières années, la promotion de l'objectif de lutte contre la pauvreté sera assurée par le fait que seuls les pays pouvant prétendre aux prêts assortis de conditions de faveur consentis par la Banque mondiale auront accès aux financements au titre du MCA. La troisième année, le seuil sera relevé de façon à étendre le dispositif aux pays à revenu intermédiaire de la tranche inférieure. Les indicateurs de performance qui concernent trois grands domaines de l'action publique l'application de méthodes justes de gouvernement, l'investissement dans le capital humain et la promotion des réformes économiques -, sont au nombre de 16. Pour pouvoir prétendre au bénéfice du MCA, les pays partenaires doivent obtenir une note supérieure au score médian pour la moitié des indicateurs dans chacun de ces domaines (et ne pas faire partie des pays que les États-Unis refusent d'admettre parmi les bénéficiaires de leur aide extérieure). Les 16 indicateurs sont :

- L'application de méthodes de gouvernement justes : i) libertés civiques, ii) droits civiques, iii) pouvoir d'influence et responsabilité, iv) efficacité des pouvoirs publics, v) primauté du droit et vi) lutte contre la corruption.

- L'investissement dans le capital humain : i) réputation de solvabilité du pays, ii) hausse des prix à la consommation sur un an, iii) politique budgétaire, iv) politique commerciale, $v$ ) qualité de la réglementation et vi) délai de création d'une entreprise.

- La promotion des réformes économiques : i) dépenses de santé du secteur public rapportées au PIB, ii) couvertures vaccinales DPT3 et rougeole, iii) dépenses du secteur public en faveur de l'enseignement primaire rapportées au PIB et iv) taux de fréquentation des établissements d'enseignement primaire.

dans trois secteurs tout au plus. Une telle démarche pourrait permettre à chaque donneur d'atteindre la masse critique requise pour être plus efficace dans chaque secteur.

Les consultations avec l'administration du pays partenaire et les priorités sectorielles définies dans la stratégie nationale de lutte contre la pauvreté devraient servir à orienter les activités sectorielles des donneurs. Dans plusieurs pays partenaires cependant, on s'aperçoit que beaucoup de membres du CAD sont actifs dans les mêmes secteurs. La mission sur le terrain effectuée au Mozambique par exemple a montré que 19 pays membres du CAD y sont présents, 14 d'entre eux ayant choisi d'axer en priorité leur action sur le secteur de la santé, 12 sur le développement rural/l'agriculture, 11 sur l'éducation et 9 sur la bonne gestion des affaires publiques. Sept pays membres du CAD ont focalisé leurs efforts sur trois de ces quatre secteurs. Pour autant, le gouvernement du Mozambique n'a 
pu trouver le soutien dont il avait besoin dans le secteur des pêcheries qui est pourtant un axe de développement potentiel important pour le pays. Dans les pays partenaires où un grand nombre d'organismes d'aide au développement sont actifs, la coordination entre donneurs sous la houlette du gouvernement du pays partenaire se révèle parfois d'autant plus nécessaire pour éviter tout risque de double emploi et veiller à ce que tous les secteurs importants soient couverts.

\section{Notes}

1. Nations unies (2003), Mise en ceuvre de la Déclaration du millénaire des Nations unies. Rapport du Secrétaire général. New York. Ref. A/58/323.

2. La Facilité de financement internationale devrait permettre de mobiliser au bénéfice de l'aide jusqu'à 50 milliards USD supplémentaires par an d'ici à 2015 en convertissant les promesses d'accroissement de l'APD qui ont été faites en engagements contraignants à long terme et en utilisant ces engagements pour émettre des obligations cotées AAA remboursables par les pays donneurs après 2015. Un tel dispositif reviendrait à accroître le montant de l'aide disponible à court terme, mais les pays donneurs ne pourraient comptabiliser les sommes supplémentaires ainsi dégagées dans leur APD tant qu'ils n'auraient pas commencé à rembourser les obligations, c'est-à-dire pas avant 2015.

3. Ministère des Affaires étrangères du Danemark (2003), A World of Difference, Copenhague.

4. OCDE (2003), L'efficacité de l'aide et la sélectivité : intégrer de multiples objectifs dans les décisions d'affectation de l'aide. OCDE, Paris. 


\section{Chapitre 5}

\section{Profil général des systèmes de coopération pour le développement des pays membres du CAD}

On observe des différences sensibles et notables dans la manière dont les pays membres du CAD s'organisent pour gérer et mettre en œuvre leurs programmes de coopération pour le développement. Ces différences sont perceptibles au niveau de l'implication, plus au moins grande, du ministère des Affaires étrangères, de la présence et de la fragmentation institutionnelles dans les pays partenaires et des relations entre les bureaux sur le terrain et le siège. Il semble qu'il n'y ait guère de corrélation entre le volume des programmes d'aide extérieure bilatéraux qui sont gérés et les stratégies qui sont suivies. Les programmes de coopération pour le développement s'articulent autour d'une structure très évolutive : beaucoup de programmes par pays mis en œuvre par des membres du CAD font l'objet à intervalles réguliers de réformes organisationnelles déclenchées par des événements tels que des changements dans l'équipe de direction ou des réorientations affectant le ciblage ou l'envergure des programmes. Quel que soit le mode d'organisation choisi, la mise en place de structures sur le terrain propres à améliorer l'efficacité de la gestion de l'aide et à contribuer à la réalisation des objectifs visés dans de bonnes conditions d'efficience et d'efficacité deurait être la préoccupation centrale. 


\section{Profil des systèmes de coopération pour le développement}

L'une des principales raisons qui expliquent les variations observées d'une structure à l'autre tient au fait que les programmes d'aide demeurent tributaires des considérations politiques qui prévalent à l'échelon national, des systèmes de gouvernement et des pratiques en vigueur dans la fonction publique, y compris des politiques suivies en matière de ressources humaines, propres à chaque nation. Des options sont parfois exclues par certains pays alors que pour d'autres, elles font partie des solutions envisageables. La Constitution irlandaise par exemple impose que le nombre de ministres du gouvernement ne dépasse pas quinze, obligation qui a une incidence sur la marge de manœuvre dont dispose le pays pour créer une administration spécifiquement chargée de la coopération pour le développement placée sous la responsabilité d'un ministre. En Suède, les ministères supervisent les activités d'un certain nombre d'organismes qui leur sont rattachés et qui ont pour mission de faire appliquer les lois votées par le Parlement. Cette conception de l'administration explique pourquoi il existe en Suède un organisme d'exécution chargé de la coopération pour le développement qui ne dépend pas du ministère des Affaires étrangères, et pourquoi cette structure devrait selon toute probabilité être maintenue. De même, les réformes de la fonction publique en général engagées dans chaque pays peuvent voir des répercussions profondes sur les programmes d'aide, comme on a pu le constater au moment de l'adoption par certains pays, dans le cadre d'initiatives engagées à l'échelle de l'ensemble de l'administration, de systèmes de gestion fondés sur les résultats. Comprendre le fonctionnement d'un système de coopération pour le développement exige de connaître les domaines dans lesquels les pays ont la flexibilité requise pour faire évoluer leurs structures et leurs méthodes et ceux dans lesquels le contexte national impose des limites ou exige le respect de procédures transitoires préalables à l'introduction de changements.

Ces dernières années, on a assisté à des restructurations profondes et à des réformes radicales des systèmes de coopération pour le développement. En 2004, l'Autriche a créé un nouvel organisme d'exécution qui ne dépend pas de son ministère des Affaires étrangères tandis que la Norvège, pour sa part, a réintégré une bonne partie des missions assignées à l'organisme d'exécution dans les attributions du ministère des Affaires étrangères. Des études des services chargés de la coopération pour le développement au sein des ministères des Affaires étrangères de l'Irlande et de la Nouvelle-Zélande ont conduit la première à décider de renforcer la structure existante et la seconde à créer un nouvel organisme chargé de la coopération pour le développement jouissant d'un statut de semi-autonomie vis-à-vis du ministère des Affaires étrangères et du Commerce international. Les États-Unis ont institué de leur côté le Millennium Challenge Corporation qui a vocation à gérer les fonds promis aux pays en développement qui appliquent des méthodes justes de gouvernement, investissent dans leur capital humain et encouragent la liberté économique. Cette décision signe la naissance d'un nouvel organisme dont le nom vient s'ajouter à la liste des institutions qui s'occupaient déjà d'acheminer l'aide extérieure fournie par les États-Unis (voir également encadré 5.1). 


\section{Encadré 5.1. Restructuration des systèmes de coopération pour le développement des pays membres du CAD}

Les modes d'organisation adoptés par les pays membres du CAD pour gérer leur aide extérieure sont évolutifs et souvent complexes. Ils font régulièrement l'objet de réformes en profondeur et de restructurations consistant par exemple à fusionner des instances auparavant distinctes, à créer de nouvelles entités ou à réaménager la structure interne des organismes d'aide au développement. Mais quelles sont les circonstances qui déclenchent ces mouvements de réforme?

- Un changement de gouvernement :

* En Australie, l'équipe qui a pris les rênes en 1996 a demandé la réalisation d'un examen indépendant du programme d'aide extérieure australien qui a abouti à la publication d'un rapport détaillé intitulé One clear objective: Poverty reduction through sustainable development (Un objectif clair : la réduction de la pauvreté par le développement durable). Cet exercice a confirmé l'intérêt qu'il y avait à continuer de confier la gestion du programme d'aide à un organisme d'aide au développement unique et autonome et conduit à préconiser parallèlement un certain nombre de changements au nombre desquels la mise en place d'une commission ministérielle indépendante et la création d'un Office d'examen et d'évaluation dont la vocation serait de permettre de disposer de données plus transparentes et plus fréquemment actualisées sur les résultats obtenus grâce à l'aide distribuée.

* En Nouvelle-Zélande, l'équipe gouvernementale arrivée au pouvoir en 2000 a demandé l'exécution d'une étude indépendante du programme d'aide publique au développement dont les conclusions ont été publiées sous le titre Towards Excellence in Aid Delivery (Sur la voie de l'excellence dans le domaine de l'acheminement de l'aide). Cet exercice a débouché sur la création, en 2002, de la New Zealand Agency for International Development (NZAID), qui jouit d'un statut de semi-autonomie au sein du ministère des Affaires étrangères.

- Un changement au niveau des hauts responsables :

* Au Japon, le nouveau président de l'Agence japonaise de coopération internationale (JICA) a engagé des réformes institutionnelles de grande ampleur visant à donner un nouvel élan aux programmes d'aide extérieure en intensifiant les transferts d'effectifs du siège vers les missions à l'étranger.

* En Norvège, le nouveau ministre du Développement international, nommé en 2002, a demandé une évaluation des services chargés d'administrer la politique norvégienne d'aide au développement, évaluation qui a motivé, en 2004, le transfert de certaines compétences de l'Agence norvégienne de développement et de coopération au ministère des Affaires étrangères (NORAD).

* Au Royaume-Uni, la nomination d'un nouveau Secrétaire permanent du Department for International Development (DFID) a déclenché un certain nombre de mouvements de restructuration en profondeur au sein de la Division politique, qui est passée d'une organisation en plusieurs départements spécialisés chacun dans un seul domaine, par exemple dans les domaines de l'éducation et de la santé, à une organisation reposant sur des équipes pluridisciplinaires concentrant leurs efforts sur des questions fondamentales telles que l'efficacité de l'aide et la réduction de la pauvreté dans des contextes difficiles. 


\section{Encadré 5.1. Restructuration des systèmes de coopération pour le développement des pays membres du CAD (suite)}

- Une décision d'affecter à l'aide extérieure un surcroît de ressources :

* Au lendemain de sa décision de porter son APD à 0.7 pour cent de RNB d'ici fin 2007, le gouvernement irlandais a chargé en 2001 une commission d'experts indépendants de mener à bien un examen complet de la politique et de la gestion de l'aide qui a conduit à placer deux organismes d'aide sous la tutelle du ministère des Affaires étrangères et à créer au sein du ministère un nouvel organe de surveillance de haut niveau à vocation consultative.

* En 2002, le président des États-Unis a annoncé la création d'un nouveau Millennium Challenge Account qui devrait permettre de mobiliser chaque année 5 milliards USD supplémentaires en faveur de l'aide extérieure jusqu'en 2006. Un processus de consultation et de réflexion a été engagé dans le prolongement de cette annonce pour tenter de déterminer comment gérer au mieux ces fonds et la décision de créer un nouveau Millennium Challenge Corporation a été prise.

- Des événements extérieurs de portée majeure :

* Le ministère des Affaires étrangères de la Suède a été profondément réorganisé en 1996 à la suite de l'entrée du pays dans l'Union européenne en 1995. La nouvelle structure a été conçue à l'époque pour permettre à la Suède d'aborder plus efficacement toute une série de dossiers que le ministère allait désormais être appelé à traiter.

- Des interrogations à propos de l'efficacité de l'aide :

* L'Agence autrichienne de développement (ADA) a été créée en 2004: elle est l'organisme chargé de l'exécution du programme d'aide bilatérale de l'Autriche. L'ADA a compétence pour recruter du personnel, aussi bien technique qu'administratif, afin d'améliorer l'adéquation aux circonstances et la qualité des efforts déployés par l'Autriche dans le domaine de la coopération pour le développement.

* L'Institut portugais d'aide au développement (IPAD) a vu le jour en 2003 : il résulte de la fusion de l'Institut de la coopération portugaise (ICP) et de l'Agence portugaise de soutien du développement (APAD). L'IPAD est ainsi devenu le principal organe responsable, au sein du ministère des Affaires étrangères, de la formulation et de la mise en œuvre de la politique de coopération pour le développement du Portugal. La fusion opérée avait pour objectif d'améliorer la qualité et l'efficacité du programme d'aide extérieure du pays.

\section{Caractéristiques des systèmes de coopération pour le développement des pays membres du CAD}

\section{Implication du ministère des Affaires étrangères}

Parce qu'apporter une aide extérieure exige de collaborer avec des citoyens, des institutions et des gouvernements d'autres pays, coopération pour le développement et relations extérieures sont toujours étroitement imbriquées, ce qui implique que dans tous les pays membres du $C A D$, le ministère des Affaires étrangères joue toujours un rôle, plus ou moins important selon les cas, dans les systèmes de coopération pour le développement (voir encadré 5.2), si ce n'est au siège, du moins sur le terrain. Dans certains pays, il existe parfois un service chargé de la coopération pour le développement 
au sein du ministère des Affaires étrangères, ou bien c'est le ministère des Affaires étrangères qui assume la responsabilité première d'un aspect particulier du programme d'aide, comme l'aide humanitaire, la promotion de la démocratie et de la bonne gouvernance ou les contributions versées à certains organismes multilatéraux. Dans les pays où un certain nombre d'instances participent à l'acheminement de l'aide extérieure, le ministère des Affaires étrangères assume parfois des fonctions de pilotage ou de coordination. On rencontre quelquefois aussi des organismes jouissant d'une large autonomie pour la gestion du programme de coopération pour le développement, mais placés sous la responsabilité politique du ministère des Affaires étrangères.

Indépendamment du mode d'organisation choisi pour le siège, les liens existant entre les programmes de développement et les relations extérieures au sens large apparaissent très clairement dans les pays partenaires dans lesquels l'ambassadeur et d'autres agents des services diplomatiques jouent parfois un rôle important. L'ambassadeur représente le pays donneur au plus haut niveau et il a la possibilité d'influencer le débat dans des domaines de première importance pour la réalisation des objectifs poursuivis sur le front du développement, tels que le soutien à l'adoption de pratiques démocratiques, la promotion de la bonne gouvernance, du respect des droits de l'homme et de l'État de droit. Dans un grand nombre de pays, l'ambassadeur contribuera à la définition de la stratégie par pays du donneur ou s'attachera à la commenter, et son accord sera dans certains cas requis pour la poursuite de telle ou telle activité. Certains ambassadeurs disposent également d'un fonds qu'ils peuvent utiliser pour appuyer des actions de faible envergure en relation avec le développement ayant pris forme sur le terrain. Il est en conséquence préférable que les ambassadeurs et les diplomates en poste dans des pays en développement ou en transition possèdent un solide bagage sur les questions de développement, que celui-ci ait été acquis dans le cadre de leur formation ou grâce à des expériences antérieures, si l'on veut que leur travail appuie et intègre le programme de coopération pour le développement. Si les agents des services diplomatiques peuvent cultiver à titre personnel un intérêt pour les questions de développement, il est préférable, pour se donner les meilleures chances qu'ils aient la formation ou l'expérience voulues, de veiller à ce qu'ils viennent d'un pays où le ministère des Affaires étrangères est étroitement associé à la mise en œuvre du programme d'aide extérieure.

\section{Fragmentation institutionnelle}

La fragmentation du programme d'aide extérieure entre institutions est aussi un aspect pour lequel on observe des différences sensibles d'approche entre les pays membres du CAD. Rares sont les pays, à savoir le Danemark, la Finlande, les Pays-Bas et la NouvelleZélande, qui confient la gestion ou la coordination de la totalité ou de la majeure partie de leur aide extérieure à une seule et même institution et qui jugent utile d'assimiler l'organisme au programme de coopération pour le développement. Dans beaucoup de pays en revanche, on observe une fragmentation légère, et pourrait-on dire, gérable de l'aide entre plusieurs institutions. Cette situation résulte du fait que les contributions versées à certains organismes multilatéraux, et en particulier aux institutions financières internationales, sont financées et gérées par des services distincts, notamment en Irlande et au Royaume-Uni. Elle peut également s'expliquer par le fait que certaines institutions spécialisées de petite taille, comme l'Australian Centre for International Agricultural Research (ACIAR) ou le Centre de recherche pour le développement international (CRDI) du Canada, font partie intégrante du programme d'aide extérieure. L'Autriche, le Luxembourg, la 
Norvège et la Suède ont opté pour une autre configuration consistant à confier la mise en œuvre de leur programme d'aide à un organisme d'exécution distinct.

Dans le plupart des autres pays membres du CAD, la fragmentation institutionnelle est telle qu'il est parfois nécessaire de mettre en place des mécanismes permettant de regrouper officiellement des éléments disparates afin de veiller à la complémentarité des efforts déployés par chaque composante et de faire en sorte que les synergies éventuelles soient dûment mises à profit. La tâche n'est pas toujours aisée lorsque sont mobilisées des instances nationales, régionales et locales, lorsque certaines des institutions concernées ont de vastes missions et poursuivent de multiples objectifs en dehors de la promotion du développement, lorsque les ministères dont les actions respectives doivent être coordonnées ont une dimension et une puissance politique supérieures à celles de l'organisme coordonnateur ou encore lorsque les institutions sont installées dans des villes différentes. Lorsqu'un ministère assume la responsabilité de la coordination des activités menées par d'autres ministères, il doit être investi des compétences requises pour pouvoir remplir sa mission avec succès, c'est-à-dire qu'il doit notamment avoir le pouvoir d'approuver ou de rejeter tel ou tel projet ou de statuer sur le financement de telle ou telle activité.

Un grand nombre de pays membres du CAD s'efforcent de relever le défi en renforçant le pouvoir de coordination dont sont investis les grands ministères ou en mettant en œuvre de nouvelles méthodes (ce que font par exemple le Japon et les États-Unis, voir ci-après). En marge des dispositifs officiels, les réunions et les communications informelles entre les membres du personnel des différentes institutions constituent un instrument essentiel et complémentaire de coordination dont l'utilisation mérite d'être encouragée par les hauts responsables. Dans certains pays, des commissions interministérielles sont quelquefois mises en place soit à titre permanent, soit ponctuellement lorsqu'une question importante se fait jour.

\section{Un exemple : le Japon}

Pour coordonner le travail des nombreux organismes officiels qui composent son système de coopération pour le développement, le Japon a mis en place toute une panoplie de mécanismes. Outre des réunions au niveau ministériel entre des représentants des huit ministères intervenant dans le domaine de la coopération économique, des instruments de coordination ont été institués à pratiquement tous les niveaux d'administration. Concrètement, ces instruments se matérialisent par la tenue de réunions interministérielles entre directeurs généraux chargés, au sein de leurs ministères respectifs, de la gestion de l'APD et de réunions entre directeurs de services associés à la gestion de l'APD, réunions qui sont consacrées à des débats de fond sur l'APD et à l'élaboration de propositions devant donner lieu à des négociations lors des réunions interministérielles. Par ailleurs, un certain nombre de rencontres entre experts techniques spécialistes de questions telles que la coopération technique, l'évaluation de l'APD et la coopération financière sont organisées. 


\section{Encadré 5.2. Implication du ministère des Affaires étrangères dans la gestion des programmes d'aide à l'extérieure}

L'implication du ministère des Affaires étrangères dans la gestion des programmes d'aide est plus ou moins marquée selon les pays membres du CAD et l'on peut dégager plusieurs schémas :

- Le ministère des Affaires étrangères joue un rôle prépondérant et assume la responsabilité de la gestion de la majeure partie du programme d'aide extérieure :

* Au Danemark : l'aide extérieure du Danemark est gérée par le Groupe Sud du ministère des Affaires étrangères.

* En Finlande : l'aide extérieure de la Finlande est gérée par la direction de la politique de développement du ministère des Affaires étrangères.

* En Irlande : l'aide extérieure de l'Irlande est pour l'essentiel gérée par la direction de la coopération pour le développement du ministère des Affaires étrangères.

* Aux Pays-Bas : l'aide extérieure des Pays-Bas est gérée par la direction générale de la coopération internationale du ministère des Affaires étrangères.

- Une variante de ce système consiste à confier un rôle prépondérant à un organisme indépendant dans une certaine mesure du ministère des Affaires étrangères :

- En Australie : l'Australian Agency for International Development (AusAID) est un organisme administrativement autonome rattaché au ministère des Affaires étrangères et du Commerce extérieur.

* En Nouvelle-Zélande : le New Zealand Agency for International Development est un organe jouissant d'un statut de semi-autonomie au sein du ministère des Affaires étrangères et du Commerce international.

* En Suisse : l'aide extérieure de la Suisse est essentiellement du ressort de la direction du développement et de la coopération (DDC) du Département fédéral des Affaires étrangères. L'une des caractéristiques du programme d'aide suisse tient toutefois au fait que la Division politique IV du Département fédéral des Affaires étrangères et que le secrétariat d'État à l'Économie (SECO) apportent également une aide substantielle.

- Une autre déclinaison consiste à accorder un rôle prépondérant à un ministère ou à un organisme totalement distinct du ministère des Affaires étrangères :

* Le Canada : l'Agence canadienne de développement international (ACDI) ne dépend pas du ministère des Affaires étrangères et du Commerce international et rend compte au Parlement via le ministre de la Coopération internationale.

* Le Royaume-Uni : le Department for International Development ne dépend pas du ministère des Affaires étrangères et du Commonwealth et rend compte au Parlement via le ministre du Développement international.

- Le ministère des Affaires étrangères peut également être investi de la responsabilité globale de l'aide extérieure alors que les activités relevant de l'aide bilatérale sont menées à bien par un organisme d'exécution distinct:

- En Autriche : la direction de la coopération pour le développement et de la coopération avec l'Europe orientale du ministère des Affaires étrangères assume la responsabilité globale de l'aide extérieure autrichienne. Les projets bilatéraux sont en revanche mis en œuvre par l'Agence autrichienne de développement.

* En Belgique : la direction générale de la coopération au développement (DGDC) du ministère des Affaires étrangères, du Commerce extérieur et de la Coopération au développement assume la responsabilité globale de l'aide extérieure belge. Les activités sont mises en œuvre par 


\section{Encadré 5.2. Implication du ministère des Affaires étrangères dans la gestion des programmes d'aide à l'extérieure (suite)}

la Coopération technique belge (СТВ). L'une des caractéristiques du programme de la Belgique tient à la coexistence d'engagements effectifs mais séparés de la part des gouvernements flamands et wallons.

* Au Luxembourg : le ministère des Affaires étrangères est globalement responsable de l'aide extérieure distribuée par le pays dont l'acheminement est confié à un organisme distinct, l'Agence d'exécution de la coopération pour le développement luxembourgeoise.

* En Norvège : le ministère des Affaires étrangères assume la responsabilité globale de l'aide extérieure norvégienne, qui est en partie acheminée par l'Agence norvégienne de développement international, organisme qui est d'ailleurs une émanation du ministère des Affaires étrangères.

* En Suède : la direction du développement mondial du ministère des Affaires étrangères assume la responsabilité globale de l'aide extérieure distribuée par la Suède, celle-ci étant acheminée par les bons soins de l'Agence suédoise de coopération internationale au développement (SIDA).

- Cette solution se rencontre sous plusieurs versions, donnant lieu notamment à un partage de la responsabilité globale de l'aide extérieure revenant à confier cette responsabilité à un autre ministère ou à faire appel à une large palette d'organismes d'exécution :

* En France : les principaux acteurs du système d'aide extérieure français sont la direction générale de la Coopération internationale et du développement du ministère des Affaires étrangères et la direction du Trésor du ministère de l'Économie, des Finances et de l'Industrie. L'Agence française de Développement (AFD) est le principal organisme d'exécution des activités bilatérales menées par la France.

* En Allemagne : le ministère fédéral de la Coopération économique et du Développement (BMZ) est chargé de la planification et de la mise en œuvre de la politique allemande de coopération pour le développement. C'est un ministère distinct du ministère fédéral des Affaires étrangères et il rend compte au Parlement par la voix du ministre de la Coopération économique et du Développement. La mise en œuvre de la politique de développement de l'Allemagne est assurée par une multitude d'organisations dont la Banque pour la reconstruction (Kreditanstalt für Wiederaufbau - KfW) et sa filiale, l'Agence allemande de promotion de l'investissement et du développement (DEG), l'Agence allemande pour la coopération technique (GTZ), l'Internationale Weiterbildung und Entwicklung (InWEnt) ${ }^{*}$ dans le domaine de la formation, le Deutscher Entwicklungsdienst (DED) dans le domaine de l'envoi de volontaires. Le ministère des Affaires étrangères s'occupe pour sa part de l'aide humanitaire dont la mise en œuvre est assurée par les organisations susmentionnées.

- Au Japon : le service de la coopération économique du ministère des Affaires étrangères joue un rôle pivot, mais l'acheminement de l'aide extérieure japonaise s'effectue par l'intermédiaire de divers organismes gouvernementaux dont les principaux sont l'Agence japonaise de coopération internationale et la Banque japonaise de coopération internationale (JBIC).

- Dans les autres pays membres du CAD, le ministère des Affaires étrangères est un acteur important et peut quelquefois se voir investi d'une mission spéciale de coordination:

- En Grèce : la direction de la coopération pour le développement international du ministère des Affaires étrangères grec a une mission de coordination de l'aide extérieure bilatérale distribuée par la Grèce dont la mise en œuvre est assurée par une douzaine de ministères et organismes gouvernementaux. 


\section{Encadré 5.2. Implication du ministère des Affaires étrangères dans la gestion des programmes d'aide à l'extérieure (suite)}

* En Italie : parmi les divers ministères et instances publiques locales qui concourent à la distribution de l'aide extérieure italienne, la Direzione generale per la cooperazione e lo suiluppo du ministère des Affaires étrangères joue un rôle de chef de file pour la mise en œuvre du programme d'aide bilatérale.

* Au Portugal : la mise en œuvre de l'aide extérieure est assurée par près d'une vingtaine de ministères et organismes gouvernementaux et plus de 300 communes. Le ministère des Affaires étrangères assume la responsabilité globale de l'aide extérieure portugaise, dont la coordination est confiée à l'Institut portugais d'aide au développement.

* En Espagne : le secrétariat d'État pour la Coopération internationale et l'Amérique latine (SECIPI), rattaché au ministère des Affaires étrangères, et l'organisme d'exécution qui en dépend, l'Agence espagnole de coopération internationale (AECI), sont des acteurs de premier plan du système espagnol d'aide extérieure qui englobe également le ministère de l'Économie et diverses régions et communes autonomes.

- Aux États-Unis : en dehors du canal de l'USAID, l'aide extérieure distribuée par les États-Unis est acheminée par un large éventail d'institutions fédérales au nombre desquelles le Département d'État, le Département du Trésor, le ministère de la Santé, le Millennium Challenge Corporation et Peace Corps. Le secrétaire d'État a la responsabilité, au niveau gouvernemental, des activités du Département d'État et de l'USAID et il préside le conseil d'administration du Millennium Challenge Corporation.

* InWEnt est née en 2002 de la fusion de la Carl Duisberg Society (CDG) et de la Fondation allemande pour le développement international (DSE).

\section{Un exemple : les États-Unis}

De tous les membres du CAD, les États-Unis sont celui qui gère le programme d'aide extérieure le plus important en volume; ils font appel à une multitude d'institutions pour acheminer leur aide. La coordination entre ces différentes institutions est en conséquence fondamentale pour qu'ils puissent optimiser l'impact global de leurs activités en faveur du développement. Au niveau stratégique, la National Security Strategy adoptée en 2002, qui fait sans ambiguïté du développement la clé de voûte de la sécurité nationale des États-Unis et insiste sur l'intérêt stratégique de la coopération pour le développement, aux côtés de la défense et de la diplomatie, plaide en faveur de la coordination. Cette conception est relayée dans le premier Plan stratégique pour la période 2004-2009, établi conjointement par le Département d'État et l'USAID, qui assigne quatre grands objectifs stratégiques aux deux instances placées sous la responsabilité du Secrétaire d'État. Ces objectifs, qui doivent être atteints par l'une et l'autre instance tant au niveau global qu'au niveau de chacune de leurs émanations, y compris au niveau des bureaux à l'étranger, sont les suivants : i) assurer la paix et la sécurité, ii) progresser sur la voie de l'instauration d'un développement durable et de la prise en compte des intérêts mondiaux, iii) promouvoir la compréhension mutuelle au niveau international, et iv) renforcer les capacités des services diplomatiques et les programmes. Pour veiller à la mise en œuvre du Plan stratégique, un Comité de gestion et un Comité directeur conjoints ont été mis en place, chacun se composant de hauts responsables des deux instances. En outre, 
les contacts et les échanges entre les deux instances sont encouragés grâce à des initiatives se concrétisant par exemple par des sessions de formation conjointes et des échanges de personnel.

\section{Présence dans les pays partenaires et relations avec le siège}

Si la plus grande partie de l'aide extérieure bilatérale distribuée par les pays membres du CAD est acheminée vers les pays partenaires, c'est bien le siège qui assure la gestion globale des fonds. La présence sur le terrain est en conséquence cruciale pour faire le lien entre le siège et les pays partenaires, en particulier si l'on souhaite améliorer l'efficacité de l'aide extérieure en alignant les politiques, les procédures et les pratiques des donneurs sur celles des pays partenaires. La présence des pays membres du CAD sur le terrain peut revêtir des formes diverses, comme on peut le constater dans le cas du Mozambique. Sur les 19 pays membres du CAD présents dans ce pays, huit seulement ont des missions sur le terrain qui font partie intégrante des services de l'ambassade. À chaque fois, on peut observer que le ministère des Affaires étrangères joue un rôle prépondérant dans la gestion ou la coordination du programme de coopération pour le développement et que le système de coopération pour le développement est globalement organisé de telle manière qu'il ne comporte pas d'organisme d'exécution distinct. Les 11 autres pays installent leurs missions sur le terrain dans des locaux séparés : soit l'organisme d'exécution du programme de coopération pour le développement est hébergé en dehors des locaux de l'ambassade, soit l'ambassade se trouve dans un autre pays. Quelle que soit la configuration retenue sur le terrain, les agents en poste au siège, qu'il s'agisse de hauts responsables, de chefs de bureaux ou de spécialistes de tel ou tel secteur, prêtent leur concours au personnel de terrain et effectuent régulièrement des missions dans les pays partenaires.

Dans les pays partenaires, l'organisation du personnel est elle aussi très variable, beaucoup de pays membres du CAD, si ce n'est tous, ayant la possibilité d'envoyer dans leurs principaux pays partenaires des agents affectés à des missions de coopération pour le développement. Dans certains cas, lorsque le programme de coopération pour le développement à gérer est de dimension relativement modeste, il se peut qu'il suffise de mobiliser au siège une seule personne à laquelle sont parfois confiées en plus d'autres missions. Les programmes plus étoffés exigent des effectifs plus nombreux et certaines missions sur le terrain emploient parfois plus d'une centaine de professionnels. Il arrive, mais rarement, en particulier dans des pays qui ne font pas partie des principaux partenaires du donneur ou dans lesquels l'organisme d'aide au développement ne dispose pas des moyens ou du pouvoir d'envoyer sur le terrain des agents du siège, la gestion du programme est confiée à une personne déjà en poste à l'ambassade, généralement un membre du personnel diplomatique, mais parfois aussi un attaché commercial, ce qui risque de générer une certaine confusion quant à la véritable finalité du programme d'aide extérieure. Dans un petit nombre d'autres cas, c'est une personne recrutée au niveau local pour une durée déterminée qui assure la gestion du programme d'aide extérieure. Cette solution présente l'avantage de permettre que la gestion du programme soit assumée par une personne compétente et expérimentée, le revers de la médaille étant qu'elle ne contribue pas à la formation ni au maintien, au sein du système de coopération pour le développement, d'un vivier de professionnels expérimentés. La plupart des pays membres du CAD procèdent également à des recrutements au niveau local, les personnes engagées par les missions sur le terrain étant de plus en plus souvent appelées à occuper des postes de spécialistes, voire de temps à autre des postes d'encadrement. 
Indépendamment de la configuration sur le terrain, le personnel, au siège comme sur le terrain, apporte une contribution importante à la formulation et à la mise en œuvre des projets et programmes bilatéraux des pays membres du CAD qui se doivent de concorder dans les grandes lignes avec les stratégies et les politiques de chaque pays. Pourtant, on observe des différences notables entre les pays en ce qui concerne le contenu des responsabilités confiées aux missions sur le terrain et l'étendue des pouvoirs conférés aux représentants sur le terrain. Ces différences sont peut-être dans une certaine mesure fonction du nombre de personnes chargées, sur le terrain, de la gestion du programme et de l'expérience qu'elles possèdent dans le domaine du développement. La mission réalisée au Mozambique a permis de mettre en évidence que la moitié des pays membres du CAD ont des systèmes plutôt centralisés dans lesquels les missions sur le terrain exécutent les décisions prises au siège et n'ont guère de marge de manœuvre pour modifier les programmes ou les financements. Les pays suivant une conception opposée autorisent certaines missions sur le terrain à élaborer et mettre en œuvre des programmes, sous réserve d'une approbation globale du siège, et à procéder à des changements au niveau des financements dans les limites d'un cadre par pays établi à l'issue d'un processus itératif auquel sont associés des membres du personnel du siège et des agents en poste sur le terrain. Dans un petit nombre de pays dont le Danemark (voir ci-après), l'évolution vers une décentralisation plus marquée s'est amorcée il y a peu et commence juste à être suivie d'effets. La mission effectuée au Mozambique a en outre montré que, la plupart du temps, les processus de décentralisation se traduisent par des changements dans les méthodes de travail des missions sur le terrain, et en particulier par une diminution des comptes rendus systématiques au siège, et qu'ils se déroulent sans évolution notable du niveau hiérarchique des effectifs en poste dans les missions sur le terrain. À l'appui de la décentralisation, une modernisation du matériel de communication, et notamment l'acquisition d'installations de vidéoconférence, pourrait être envisagée.

\section{Un exemple : le Danemark}

Désireux d'améliorer l'efficacité de son aide extérieure, le Danemark a continué à déléguer davantage de responsabilités à ses missions dans les principaux pays partenaires. Cette politique a eu des répercussions sur son système de coopération pour le développement tout entier. Les missions sont désormais clairement désignées comme les pivots des programmes par pays et elles assument la responsabilité de la gestion et du suivi des programmes, conformément aux dispositions des accords conclus chaque année entre l'ambassadeur et le siège. Elles sont chargées de recenser, de définir et de mettre en œuvre les activités, les services techniques consultatifs du siège se contentant désormais d'approuver les actions projetées et de procéder à des évaluations. Le niveau de responsabilité et le nombre d'agents dans les divisions géographiques au siège ont été réduits, les principales fonctions de ces services consistant à présent à donner leur forme finale aux documents exposant la stratégie de chaque pays et à organiser des consultations à haut niveau avec des représentants des pays partenaires. Un nouveau service de l'assurance-qualité a été créé au siège : il a vocation à exercer un suivi des accords négociés chaque année entre les ambassadeurs et le siège, à concourir au renforcement des compétences des agents en poste sur le terrain et à mener à bien des analyses de performances. Le Danemark a par ailleurs mis au point un schéma directeur pour la gestion des effectifs des missions sur le terrain 
qui exige l'envoi d'un plus grand nombre d'agents dans les pays partenaires et un recours accru à des personnes recrutées localement. Ce schéma directeur propose un modèle composé i) de l'ambassadeur et/ou d'un adjoint au responsable de la mission, ii) d'un expert danois et d'un expert recruté localement pour chaque secteur du programme par pays, iii) d'un expert danois en macro-économie, iv) d'un spécialiste danois et d'un spécialiste recruté localement des réformes institutionnelles, et $v$ ) d'un responsable financier danois. Pour un programme par pays classique s'articulant autour de trois secteurs prioritaires, ce modèle exige une équipe composée de douze professionnels dont huit venant de Copenhague.

\section{Aide extérieure fournie par des instances infranationales}

Dans certains pays membres du CAD, on a constaté ces dernières années une participation croissante des instances infranationales (régions, provinces et communes) au financement et à la mise en œuvre des activités menées au titre de l'aide extérieure. Cette forme d'aide au développement, que l'on qualifie parfois de coopération décentralisée, est surtout développée en Allemagne, en Autriche, en Belgique, au Canada, en Espagne, en France, en Italie et au Portugal (voir exemples ci-après). Dans certains pays, dont l'Espagne, la France et l'Italie, la mobilisation des instances infranationales s'inscrit dans un cadre législatif adopté par le gouvernement ou résulte d'une politique menée par l'organisme national d'aide au développement.

\section{Un exemple : le Portugal}

$\mathrm{Au}$ Portugal, vingt-deux districts et plus de 300 communes prennent part aux activités menées au titre de l'aide extérieure, souvent dans des pays lusophones comme le Cap-Vert où la coopération entre communes constitue un volet important de l'effort de coopération pour le développement déployé par le Portugal. Mettant à profit les liens établis grâce à la communauté capverdienne installée au Portugal, bon nombre des 17 communes du Cap-Vert entretiennent des relations de coopération directes avec des villes portugaises dans des domaines tels que l'éducation, la culture, le renforcement des institutions locales, la conservation du patrimoine et le bien-être social. Ce type de coopération est généralement très appréciée par les communes du Cap-Vert car elle peut leur offrir le moyen de se procurer rapidement des ressources pour financer des projets locaux, comme la construction de bibliothèques, d'écoles ou d'installations sportives. Dans la plupart des cas, en particulier lorsqu'il existe un besoin de financement urgent, elles entrent directement en contact avec les communes portugaises qui sont leurs partenaires sans passer par les autorités capverdiennes ou par l'ambassade du Portugal à Praia.

\section{Un exemple : l'Espagne}

Le gouvernement régional de Valence dispose d'un budget non négligeable pour financer son programme de coopération pour le développement, mis en place officiellement en 1999. La Direction de la coopération internationale sise à Valence assure la gestion de l'intégralité programme d'aide de la région. En 2002, le Comité de Valence pour l'aide humanitaire et l'aide d'urgence a été créé afin d'améliorer la coordination entre les différentes parties prenantes en présence. Le montant des ressources disponibles dans la région, en particulier des ressources 
affectées à l'aide en cas de catastrophe naturelle, allant croissant, il a fallu centraliser les flux. Le Comité, composé de représentants des administrations locales, de deux banques, d'ONG, du Fonds de solidarité de la Communauté de Valence et de la Fédération des communes de la région de Valence, se réunit dans les 48 heures suivant une crise et prend des décisions d'intervention immédiate en concertation avec l'Agence espagnole de coopération internationale (AECI). Il envoie ensuite une délégation chargée de recenser les secteurs les plus touchés et de consulter les interlocuteurs locaux et les organisations espagnoles concernées.

Un certain nombre d'arguments plaident en faveur de la participation des instances infranationales aux activités relevant de l'aide extérieure. En premier lieu, elle accroît le volant de l'aide consenti par un pays puisque les prélèvements fiscaux effectués au profit des instances infranationales peuvent ainsi être mobilisés au bénéfice d'activités de coopération pour le développement. En Espagne par exemple, environ 15 \% l'APD sont financés par des instances infranationales. En deuxième lieu, d'aucuns estiment que le canal des instances infranationales permet aux citoyens de se sentir plus étroitement associés aux activités en faveur du développement et favorise donc une prise de conscience dans l'opinion et une meilleure compréhension des enjeux du développement. Enfin, les jumelages entre institutions homologues à différents niveaux d'administration sont considérés comme bienvenus car ils facilitent les échanges, dans le cadre d'une relation s'inscrivant dans la durée, de connaissances, de compétences et de savoir-faire.

Certains points faibles de cette forme d'aide extérieure ont toutefois été mis au jour*. Il se révèle difficile pour les pays partenaires de traiter avec un grand nombre de donneurs et l'entrée en scène d'intervenants nouveaux ou différents complique encore les efforts de coordination et d'appropriation à l'échelon local. En ce qui concerne le personnel, les instances infranationales sont souvent mal dotées et ne disposent ni des effectifs, ni des compétences requises. Les mécanismes de suivi et d'évaluation sont peu nombreux et, lorsqu'il en existe, ils sont relativement médiocres. La communication d'informations sur les activités menées, qui faciliterait la coordination, est souvent mal organisée. Les pays membres du CAD sont de plus en plus conscients des lacunes inhérentes aux actions menées par des instances infranationales et commencent à prendre les mesures qui s'imposent. En France par exemple, les administrations locales s'emploient à engager à un certain nombre de réformes pour améliorer leurs performances, notamment en créant une base de données centralisant des informations sur les diverses activités menées, en recensant les bonnes pratiques dont il convient de s'inspirer lorsqu'on opte pour cette forme de coopération pour le développement et en mettant au point des instruments communs, en particulier dans les domaines du suivi et de l'évaluation.

\footnotetext{
* Desmet, A. et P. Develtere (2002), Sub-national Authorities and Development Co-operation in the OECD-DAC Member Countries, Hoger instituut voor de arbeid, Louvain.
} 



\section{Chapitre 6}

\section{Gestion des organismes d'aide : structures et systèmes internes}

D'une complexité qui s'accentue au fil du temps, la coopération pour le développement couvre des aspects à la fois plus divers et interdépendants, placés sous l'autorité de plusieurs départements ou ministères, de sorte que la question de la structure interne la mieux adaptée aux organismes d'aide devient de plus en plus préoccupante et de plus en plus difficile à résoudre. Les principaux défis auxquels doivent faire face les structures et systèmes internes des pays membres du CAD sont les suivants: la nécessité d'une plus grande coordination, le besoin accru de compétences spécialisées et de soutien technique, l'évolution des priorités et l'émergence de questions pluridisciplinaires à prendre en compte dans toute l'organisation. 


\section{Schéma d'organisation}

Lorsque les premiers pays membres du CAD ont mis en place leurs systèmes de coopération pour le développement dans les années 60, il était habituel et peut-être justifié de compartimenter la gestion de l'aide, qui était assurée par des unités structurelles distinctes disposant d'une grande autonomie. Les contributions aux agences multilatérales et l'aide humanitaire étaient gérées par des services spéciaux qui ne se trouvaient pas forcément dans les mêmes locaux que l'organisme d'aide. Les départements et sièges régionaux puissants pouvaient gérer des programmes bilatéraux et disposer d'agents affectés aux bureaux géographiques des principaux pays partenaires et de représentants sur le terrain, essentiellement chargés de relayer l'information entre le siège et le pays partenaire. Seul un nombre limité de domaines, comme les infrastructures, la santé et l'éducation, exigeait des compétences spécialisées. Les questions pluridisciplinaires étaient peu nombreuses et l'idée d'une prise en compte systématique du développement dans l'élaboration des politiques encore peu répandue.

Dans les décennies qui ont suivi, la coopération pour le développement est devenue plus complexe et les organisations ont dû adapter leurs structures, à la fois pour couvrir un plus large éventail de sujets et pour que chaque unité puisse travailler en coordination et en complémentarité avec les autres. L'apparition des stratégies de lutte contre la pauvreté, par exemple, nécessite une plus grande coordination entre les unités responsables des établissements financiers internationaux et celles qui gèrent les programmes d'État à État. Les activités bilatérales peuvent maintenant être mises en œuvre au moyen de contributions préaffectées à des organismes multilatéraux (assistance " multibilatérale »). L’adoption de stratégies de partenariats pour l'acheminement de l'aide extérieure a conduit certains membres du CAD à transférer l'essentiel de leurs programmes bilatéraux à d'importantes unités situées dans chacun des principaux pays partenaires, au point de ne plus conserver au siège, dans leurs départements régionaux, qu'un personnel très réduit. De plus en plus, l'aide humanitaire fournie à court terme est appelée à évoluer vers des programmes de redressement à moyen terme et éventuellement vers des partenariats de développement à long terme. Différents aspects, comme la problématique homme-femme ou le sida, maintenant intégrés aux programmes de développement, exigent le recrutement de spécialistes qui puissent travailler en collaboration avec les autres agents chargés de la mise en œuvre de l'aide bilatérale et multilatérale, des dispositifs de cofinancement des ONG et de l'aide humanitaire.

À une époque, les organismes d'aide des pays membres du CAD avaient habituellement une structure géographique plutôt que fonctionnelle, mais elles ont souvent été conduites, du fait de la complexité de la lutte contre la pauvreté, à adopter une structure hybride, en accordant une place dominante aux départements géographiques et multilatéraux, mais en leur fournissant selon les besoins un solide soutien sectoriel et technique (voir plus loin). Cette évolution témoigne de l'attention accrue portée à la programmation par pays pour guider les efforts des pays partenaires et de l'orientation 
Graphique 6.1. Agence australienne pour le développement international (AusAID), organigramme simplifié

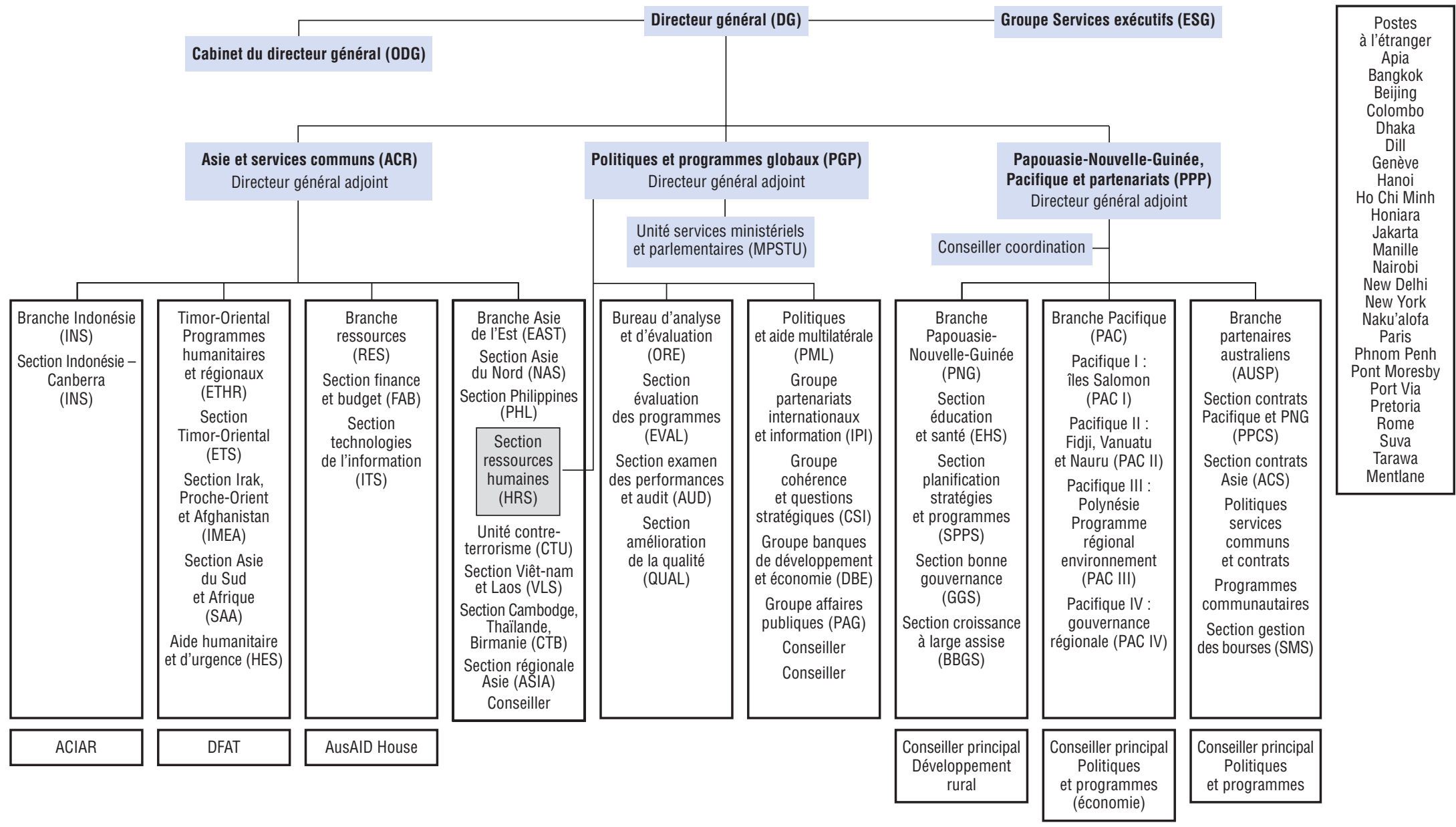

Source : AusAID (avril 2004). 
nouvelle adoptée dans certains pays membres du CAD en faveur de l'aide-programme plutôt que de projets d'assistance, et en faveur d'activités planifiées et mises en œuvre dans un cadre sectoriel.

\section{Un exemple : l'Australie}

L'Agence australienne pour le développement international gère l'essentiel de l'aide extérieure de l'Australie. Sa structure donne un rôle prééminent aux sections géographiques et multilatérales, appuyées cependant par des dispositifs de soutien sectoriel et technique (voir la graphique 6.1). Les questions de gestion et d'organisation font partie des responsabilités fondamentales de la direction, qui procède régulièrement à des ajustements de la structure de l'Agence pour répondre aux évolutions de la situation. À la tête de l'Agence se trouve un directeur général, responsable de son administration auprès du secrétaire parlementaire au Département des affaires étrangères et du commerce extérieur. Le directeur général est aidé par le bureau exécutif d'AusAID, qui comprend aussi trois directeurs généraux adjoints, et se concentre sur la détermination des orientations stratégiques et les questions de gestion au sens large. Le bureau exécutif est à son tour soutenu par trois comités exécutifs dont les activités concernent : i) la gestion des services communs et les questions d'organisation; ii) l'assurance de la qualité dans tous les domaines dans lesquels une aide est fournie et iii) les réalisations et les stratégies des pays partenaires.

\section{Un exemple : les États-Unis}

L'Agence des États-Unis pour le développement international gère plus de la moitié de l'APD du pays et plus des deux tiers de l'aide bilatérale au titre de la coopération. Elle a des agents en poste dans 70 pays partenaires. Son siège de Washington possède une structure hybride constituée de 10 bureaux : quatre bureaux géographiques pour : i) l'Afrique, ii) l'Asie et le Proche-Orient, iii) l'Amérique latine et les Caraibes et iv) l'Europe et l'Eurasie ; trois bureaux fonctionnels pour i) la santé dans le monde, ii) la croissance économique, l'agriculture et le commerce, et iii) la démocratie, les conflits et l'aide humanitaire ; trois bureaux supplémentaires se chargent des tâches administratives essentielles : i) gestion, ii) affaires juridiques et publiques et iii) coordination des politiques et des programmes.

\section{Nécessité accrue de communication et de coordination à l'intérieur des organismes d'aide}

La question de la communication et de la coordination internes est devenue déterminante pour une meilleure gestion de l'aide dans tous les organismes de coopération, qui s'efforcent d'améliorer leurs performances à cet égard. Beaucoup d'entre eux disposent maintenant de systèmes de communication mondiale grâce à la messagerie électronique par satellite, même si l'accès aux services de courrier électronique et d'intranet est parfois limité pour les agents en poste dans certains pays membres du CAD dépendant de fournisseurs d'accès locaux. La visite sur le terrain effectuée au Mozambique a permis de constater que certaines ambassades devaient encore appeler le siège plusieurs fois par jour pour télécharger leurs messages électroniques. Elle a également fait apparaître que des membres du CAD utilisent maintenant des systèmes de vidéoconférence pour la communication entre le siège et les bureaux locaux. Cette technologie, employée surtout par les pays à forte délégation des responsabilités et lorsque 
les échanges se font entre des fuseaux horaires proches, répond à des objectifs variés : entretiens d'embauche, réunions hebdomadaires avec les dirigeants au siège, participation à des réunions de comités d'évaluation des projets et communication avec les bureaux régionaux.

La promotion de la coordination interne peut être difficile, en particulier dans les grands organismes d'aide qui comptent plusieurs milliers d'agents en poste dans le monde entier, et peut poser plus de problèmes que la communication entre les différents ministères des pays de taille modeste. Pour la faciliter, il est possible de mettre en place des mécanismes officiels de coordination, en créant par exemple des équipes spéciales composées d'agents de différentes unités réunies pour travailler ou réfléchir ensemble sur des questions essentielles. Comme les différents lieux d'affectation du personnel des organismes d'aide ne se trouvent pas toujours dans les mêmes fuseaux horaires, il peut s'agir d'équipes « virtuelles » qui communiquent par Intranet ou par courrier électronique. Les réunions et les échanges informels entre les agents constituent un autre mode de coordination. Ils peuvent être encouragés par divers moyens, tels que cours de formation, ateliers et rencontres rassemblant les agents qui travaillent sur des problèmes connexes ou dans des pays voisins.

\section{Unités spécialisées et compétences techniques}

Les organismes d'aide doivent avoir accès à des compétences techniques spécialisées dans de nombreux domaines pour soutenir et maintenir la qualité des activités qu'ils soutiennent. Ces compétences peuvent être très spécifiques (VIH/sida par exemple), ou plus générales et nécessaires d'un bout à l'autre de la mise en œuvre du programme, comme celles qui concernent l'évaluation. La question des compétences techniques suscite des problèmes délicats pour les organismes d'aide. Il faut en particulier déterminer si le personnel doit être en poste sur le terrain ou au siège, recruter une masse critique de personnes possédant des compétences actualisées dans un ensemble de domaines spécialisés, et les retenir.

Pour répondre à ce besoin de compétences internes, certains organismes d'aide se dotent d'unités techniques ou spécialisées. Le Danemark, l'Irlande, l'Espagne et la Suède ont ainsi créé des unités techniques dont le personnel possède des compétences spécialisées dans des secteurs clés du programme d'aide. Dans d'autres pays membres du $\mathrm{CAD}$, le personnel technique peut être affecté à des départements régionaux et rattaché à des pays spécifiques. Les grands organismes d'aide peuvent employer des spécialistes à la fois au siège et dans les bureaux dont ils disposent dans les principaux pays partenaires, qui peuvent rassembler des experts venus du pays donneur ou recrutés localement. Les organismes de taille plus modeste n'ont pas nécessairement les moyens d'employer des experts dans chaque secteur clé de leur programme et dans chaque pays partenaire. Le personnel technique peut alors se voir confier plusieurs secteurs d'activités ou se consacrer à un secteur particulier dans plusieurs pays partenaires.

Souvent, le personnel technique est insuffisant dans certains domaines ou secteurs essentiels. Pour y remédier, certains membres du CAD ont choisi de recruter des experts techniques sous contrats de durée déterminée ou dans le cadre de missions de consultation. Cette solution permet aux organismes d'aide de s'assurer du renouvellement régulier des compétences techniques qu'ils utilisent, mais peut aussi aboutir à une forte rotation de personnel préjudiciable au maintien de la mémoire institutionnelle. Une autre 
solution consiste à négocier des contrats de plusieurs années avec des organismes de recherche ou des établissements d'enseignement supérieur et d'avoir recours selon les besoins aux compétences spécialisées qu'ils possèdent dans des domaines variés, et qui peuvent couvrir des aspects sectoriels mais aussi d'autres domaines comme l'appui aux programmes, la recherche ou l'évaluation.

\section{Questions pluridisciplinaires}

Certaines questions pluridisciplinaires revêtent une importance fondamentale pour la réalisation des objectifs globaux de développement. La lutte contre la pauvreté ou l'égalité des sexes, par exemple, sont des questions pluridisciplinaires, car elles influent sur les résultats et les incidences de tous les aspects du programme d'aide et ne peuvent faire l'objet d'activités autonomes ni être traitées comme des secteurs à part entière. La plupart des membres du CAD ont défini trois ou quatre aspects ou thèmes pluridisciplinaires qui se trouvent au cœur de leur programme d'aide. Les questions pluridisciplinaires auxquelles les pays membres du CAD s'intéressent le plus sont le renforcement des capacités, la prévention des conflits, la démocratie, l'égalité homme-femme, la bonne gouvernance, l'environnement, les droits de l'homme et la lutte contre la pauvreté. Dans plusieurs pays comme le Canada, le Danemark, l'Irlande et le Royaume-Uni, le VIH/sida fait partie des principaux aspects pluridisciplinaires traités.

Comme pour les questions sectorielles, les organismes d'aide peuvent avoir besoin de compétences spécialisées dans la gestion des questions pluridisciplinaires, pour que celles-ci soient bien prises en compte dans leur programme d'aide. Beaucoup de pays membres du CAD ont mis en place des unités spécialisées ou nommé des experts internes chargés des questions pluridisciplinaires. Ils ont ainsi tendance à faire porter toute la responsabilité de ces questions par des experts ou des unités techniques, de sorte qu'elles risquent de ne pas être bien prises en compte dans le cadre des programmes. Une solution plus adaptée consisterait à disposer d'experts techniques, tout en formant l'ensemble du personnel au traitement des questions pluridisciplinaires et en lui en confiant la responsabilité, pour qu'elles constituent un aspect essentiel de son travail.

La formation du personnel est donc essentielle à la gestion des questions pluridisciplinaires. Les programmes de formation doivent inclure des agents chargés de la gestion et des politiques, en poste au siège et sur le terrain, et englober un éventail de compétences adapté à différentes fonctions. La formation ne doit pas se limiter à des actions de sensibilisation, mais porter aussi sur les facultés d'analyse, les compétences en matière d'information, le suivi et l'évaluation. Différents instruments ont été conçus par les organismes d'aide et le CAD pour appuyer les efforts réalisés par les membres face aux questions pluridisciplinaires. Par exemple, le Réseau du CAD sur l'égalité homme-femme a mis au point un ensemble de fiches thématiques* qui expliquent dans quelle mesure et pourquoi l'égalité homme-femme revêt une importance décisive dans toutes les activités de développement. Ces fiches couvrent un vaste éventail de secteurs et de questions en relation avec le développement, du financement à l'évaluation ou à la gouvernance. L'annexe A.4 de ce rapport donne des indications au personnel des organismes d'aide sur la prise en compte des questions pluridisciplinaires dans les activités de développement.

\footnotetext{
* Les fiches thématiques (Gender Tip Sheets) peuvent être consultées à l'adresse suivante: www.oecd.org/cad/genre.
} 
Pour assurer la cohérence des programmes, les organismes d'aide doivent s'assurer que les ONG, les sous-traitants, les consultants et les autres partenaires de la mise en œuvre tiennent compte eux aussi des questions pluridisciplinaires dans leurs politiques et leurs activités de terrain. L'Irlande, par exemple, a mis en place un mécanisme de financement à court terme pour le renforcement de la capacité institutionnelle des ONG à prendre en compte le VIH/sida.

Deux approches différentes peuvent être adoptées face aux questions pluridisciplinaires. Une question peut être intégrée à un programme de développement et s'inscrire ainsi dans le cadre théorique des activités de l'organisme d'aide, les politiques et programmes étant ensuite adaptés en conséquence. En revanche, la prise en compte systématique d'une question pluridisciplinaire a pour objet de veiller à ce que le processus d'analyse, les politiques de développement, la planification du développement et les activités témoignent de l'importance de la question. Le programme est, dans une certaine mesure, transformé par la question pluridisciplinaire, de sorte que toutes les décisions en sont influencées et en tiennent pleinement compte.

Les principaux facteurs de succès de la prise en compte systématique d'une question pluridisciplinaire (voir encadré 6.1) montrent qu'elle nécessite un investissement considérable de la part des organismes d'aide. Ceux-ci ne peuvent donc prendre en compte un grand nombre de questions pluridisciplinaires. Il paraît réaliste de définir une ou deux questions correspondant aux objectifs globaux de l'action publique, à prendre en compte systématiquement dans tous les aspects du programme. Deux ou trois questions pluridisciplinaires de plus pourraient alors être intégrées à l'ensemble du programme. L'intégration de ces questions aux politiques et procédures exigerait des ressources, des compétences et des engagements, même à un degré moindre.

\section{Encadré 6.1. Principaux facteurs de succès de la prise en compte systématique des questions pluridisciplinaires}

Différents pays membres ont répertorié les facteurs de succès de la prise en compte systématique des questions pluridisciplinaires, qui montrent que cette approche nécessite un investissement important de la part des organismes d'aide. Ces facteurs de succès sont les suivants :

- L'engagement institutionnel doit se manifester par des politiques explicites et par l'affectation de ressources.

- Les hauts responsables doivent s'engager et jouer un rôle pilote.

- Les politiques et procédures adoptées témoignent de l'importance de la question.

- Le personnel est formé à un vaste éventail de compétences en rapport avec la question.

- La responsabilité de la question incombe à l'ensemble du personnel et un soutien technique spécialisé est reçu.

- Des indicateurs de suivi en rapport avec la question sont établis. 



\section{Chapitre 7}

\section{Gestion des ressources humaines de la coopération pour le développement}

L'efficacité de la coopération pour le développement dépend de la mobilisation adéquate d'un personnel compétent et expérimenté ayant une connaissance solide du développement, en particulier sur le terrain. La gestion des ressources humaines des organismes d'aide suscite un intérêt croissant, mais il n'existe pas beaucoup de données comparables, en raison de la grande diversité des facteurs à prendre en compte, comme la nature des programmes de développement des membres du CAD, la structure des organismes d'aide, les politiques de recrutement et de sous-traitance et les conditions d'emploi. Pour la plupart des membres du CAD, assurer une gestion rationnelle des ressources humaines est une tâche difficile, car des questions anciennes viennent se combiner à des problèmes nouveaux pour former un ensemble d'une grande complexité. Les difficultés rencontrées peuvent être liées à des compressions de personnel, à l'insuffisance des effectifs, au départ à la retraite prochain du personnel de direction, à l'évolution des compétences nécessaires et au renouvellement rapide du personnel. Il n'existe pas de solution unique face à ces problèmes et à tous ceux que peuvent poser les ressources humaines, mais, compte tenu de l'importance donnée aux partenariats, à l'appropriation locale, aux méthodes fondées sur les résultats et à l'évolution des modalités d'aide, il faut de toute évidence accorder une attention plus grande qu'on ne l'a fait jusqu'à présent à la gestion des ressources humaines de la coopération pour le développement, en se fondant sur une meilleure connaissance du personnel et des compétences nécessaires ainsi que sur une planification à plus long terme des ressources humaines. 


\section{Le " système " des ressources humaines}

Les méthodes de recrutement des organismes d'aide varient beaucoup parmi les pays membres du CAD, en fonction de facteurs comme la structure de la gestion de l'aide, l'ampleur et la nature du programme et les politiques publiques générales en faveur de l'emploi. Lorsque l'organisme d'aide est indépendant du ministère des Affaires étrangères, comme c'est le cas en Australie, au Canada, au Royaume-Uni ou aux États-Unis, les ressources humaines peuvent être gérées de façon autonome et le personnel de l'organisme d'aide est habituellement composé de spécialistes de la coopération pour le développement. Il peut s'agir de fonctionnaires occupant des postes permanents ou de personnel embauché au titre de contrats de courte ou moyenne durée.

Lorsque le ministère des Affaires étrangères est responsable de la gestion de l'essentiel du programme de coopération pour le développement, comme au Danemark, en Finlande, en Irlande ou aux Pays-Bas, la gestion du personnel affecté au développement n'est pas séparée de la gestion de l'ensemble du ministère et de la fonction publique en général. Dans ce cas, le personnel des directions chargées de la coopération et même des missions à l'étranger est souvent composé de diplomates de carrière aux compétences généralistes qui peuvent n'avoir aucune formation spécialisée à la coopération pour le développement ni de compétences particulières en matière d'organisation et de gestion de contrats. Les diplomates doivent normalement assumer différentes fonctions au cours de leur carrière, et sont évalués en fonction de leurs compétences généralistes et de leur capacité d'adaptation. Pour les aider, des agents techniques spécialisés peuvent être recrutés par les unités techniques, mais souvent sous contrat. Les conditions d'emploi ainsi que les effectifs peuvent être déterminés dans le cadre de la politique globale de gestion des ressources humaines du gouvernement.

Dans les pays membres où la mise en œuvre est confiée à un organisme distinct, les ressources humaines peuvent poser d'autres problèmes. Il peut arriver que le personnel de l'organisme ne puisse être envoyé sur le terrain, ces postes étant réservés au personnel diplomatique. Le ministère et l'organisme peuvent aussi avoir tous deux des représentations sur le terrain, parfois dans des locaux séparés, ce qui constitue une source de confusion ou de chevauchements et crée un niveau supplémentaire de gestion dans les pays partenaires.

\section{Planification des ressources humaines}

Il est essentiel de bien connaître les effectifs, les compétences représentées et l'affectation géographique des personnes chargées de la gestion de l'aide au développement pour assurer une planification efficace des ressources humaines dans ce domaine. Une analyse des caractéristiques du personnel ou des compétences a été réalisée dans certains pays membres mais elle inclut rarement l'ensemble du gouvernement et ne tient pas toujours compte des agents sous contrat et des consultants. Dans la plupart des pays, plusieurs ministères et directions prennent part à différents titres aux activités d'aide au développement. Compte tenu de cette situation et de l'importance des effectifs de personnel 
contractuel et de consultants dans certains pays, il est rare que les pouvoirs publics soient en mesure de dresser un tableau complet des effectifs, des compétences et de l'expérience ou de l'affectation administrative des agents chargés des différents aspects de la gestion de l'aide au développement.

L'amélioration de la planification des ressources humaines en faveur de la coopération pour le développement prend une importance croissante dans de nombreux pays membres du CAD, en raison des défis créés par un environnement mondial dynamique. Souvent, cette planification revêt un caractère hautement politique ; elle est influencée par des contraintes budgétaires globales et doit être mise en place suffisamment longtemps à l'avance pour être efficace. Certains des aspects à prendre en compte dans le cadre de la planification sont examinés ci-dessous.

\section{La nécessité d'une masse critique de compétences en coopération pour le développement}

L'une des difficultés rencontrées dans certains pays membres du CAD, en particulier lorsque la coopération pour le développement est intégrée au ministère des Affaires étrangères, consiste à créer et à maintenir une masse critique de compétences spécialisées dans le domaine du développement. Comme indiqué plus haut, le personnel des services de coopération peut être constitué de diplomates qui changent souvent d'affectation et dont les compétences techniques et spécialisées sont limitées. Il peut en résulter de nombreuses difficultés pour les programmes à forte composante administrative, notamment les grands programmes de bourses. Cependant, les travaux de coopération sont le plus souvent distincts des fonctions de représentation et d'élaboration des politiques qui constituent l'essentiel des travaux des ministères des Affaires étrangères. Pour être efficace, la coopération pour le développement doit de plus en plus faire appel à des spécialistes de la lutte contre la pauvreté et nécessite une connaissance sociale, économique et culturelle des problèmes rencontrés au niveau local, la mise en place de réformes de la gouvernance des pays partenaires, ainsi que des projets et programmes dont la mise en œuvre soit axée sur les résultats et les produits. En outre, une connaissance des réalités du développement et des défis à relever sur le terrain est importante pour l'élaboration et politiques et la prise de décisions au siège, alors que la décentralisation accroît les besoins en personnel expérimenté et qualifié sur le terrain.

Les spécialistes du développement embauchés sous contrat de courte ou moyenne durée risquent de ne pas être incités à rester dans le système. Il faudrait en principe créer un environnement de travail qui encourage le personnel temporaire comme les agents permanents à rester et à contribuer au renforcement de la capacité institutionnelle et des compétences pratiques. Pour cela, on pourrait envisager par exemple la création d'une filière de spécialistes du développement dans le ministère des Affaires étrangères, reconsidérer le recrutement de spécialistes contractuels, offrir des contrats de plus longue durée et faire en sorte que les conditions d'emploi soient aussi attrayantes que possible, mettre l'accent sur la mise en valeur des ressources humaines ou adopter un système de gestion fondé sur les performances. Bien que certaines contraintes dues au caractère global des politiques de ressources humaines des pouvoirs publics puissent gêner la mise en œuvre de telles stratégies, il est essentiel de rechercher et d'évaluer différents moyens de répondre aux besoins de personnel spécialiste du développement. 


\section{Éventail des qualifications et perfectionnement du personnel}

Conscients de l'élargissement de l'éventail des qualifications nécessaires dans le domaine de la coopération pour le développement, en particulier pour les activités de terrain, plusieurs pays membres du CAD ont été amenés à reconsidérer et à redéfinir les profils de compétences (voir ci-dessous). Beaucoup de pays soulignent maintenant que le personnel chargé du développement doit posséder, non seulement des connaissances techniques solides, mais de bonnes capacités d'analyse, une aptitude à la réflexion stratégique, une sensibilité aux particularités culturelles, des connaissances linguistiques et une aptitude à la négociation. Les récompenses et les incitations peuvent jouer un rôle dans l'amélioration des performances du personnel dans ces domaines et certains pays membres réfléchissent à l'importance des incitations dans le contexte de la gestion du changement. Parmi les incitations non monétaires peuvent figurer le choix des missions ou des postes à l'étranger, la plus grande visibilité donnée au personnel, l'accès à des formations spéciales ou le financement de travaux de recherche. L'évolution des profils de compétences et l'adoption d'incitations influent sur la gestion des ressources humaines dans les domaines du recrutement, de la gestion des performances, des politiques de formation et dans certains cas des conditions de travail. L'examen et la gestion proactive des ressources humaines qui se consacrent au développement sont essentiels mais il faut procéder avec prudence pour éviter tout sentiment d'insécurité et tout découragement du personnel et ne pas avoir à faire face à des départs imprévus.

\section{Un exemple : l'Australie}

Le Plan stratégique d'AusAID soulignait la nécessité de définir les compétences, les connaissances et les talents dont AusAID aurait besoin dans les cinq années à venir. Une analyse des compétences a été réalisée et utilisée à l'appui des politiques de recrutement, de la gestion des performances, des méthodes d'apprentissage et de perfectionnement et de l'analyse des besoins concernant l'affectation d'agents à l'étranger. Un Cadre d'analyse des capacités permet de définir des ensembles de compétences et de connaissances correspondant à différents niveaux d'aptitudes. Ce cadre a été conçu pour faciliter l'évaluation des performances des agents, guider la fixation d'objectifs à court et à moyen terme, définir ce qui permettra d'améliorer les performances dans des domaines essentiels de compétences et de connaissances en relation avec les tâches et les aspirations professionnelles de chacun. Différentes solutions ont été définies pour répondre aux principaux besoins de compétences : recentrage des compétences, des connaissances et des talents disponibles, accompagnement permanent et autres possibilités ciblées de perfectionnement, opérations de recrutement général, opérations de recrutement spécifique et recherche de personnes au profil adapté. La nécessité de retenir les agents qui possèdent les qualités voulues est soulignée.

L'une des solutions pour faire face à l'évolution des compétences nécessaires, en dehors du recrutement, consiste à recourir à des programmes de formation et de perfectionnement du personnel. Ceux-ci sont essentiels pour le personnel en poste comme pour les nouvelles recrues, compte tenu en particulier de l'évolution des modalités d'aide et des modes de partenariat. L'importance accordée à la formation et au perfectionnement encourage aussi le personnel à rester dans l'organisation. Certains pays mettent l'accent, par exemple, sur la formation axée sur des aspects comme l'aide-programme et l'évaluation des 
risques. En Suède, le sida consacre d'importantes ressources au perfectionnement du personnel et encourage l'apprentissage permanent à l'intérieur de l'organisation.

\section{Un exemple : la Suède}

Le sida a recensé cinq types de compétences nécessaires à la création d'un environnement propice à l'acquisition de connaissances: stratégiques, professionnelles, d'apprentissage, relationnelles et fonctionnelles. Ces compétences sont réunies dans une "étoile de compétences". En outre, les rôles et les responsabilités des dirigeants et des agents du sida ont été analysés et inventoriés dans des documents. D'après la politique de gestion du sida, la tâche principale des dirigeants consiste à créer les conditions nécessaires pour que le personnel puisse élaborer, mettre en œuvre et suivre les actions. Le gestionnaire doit assumer six fonctions: explorer, communiquer, accompagner, susciter le changement, créer des possibilités d'apprentissage et prendre des décisions ; un processus d'assurance de la qualité a été mis en place pour vérifier que les gestionnaires s'acquittent effectivement de ces responsabilités.

\section{Recrutement et politique d'égalité des chances}

Plusieurs membres du CAD constatent que de nombreux agents de haut niveau quittent leurs fonctions dans les années qui précèdent la retraite. Certains ont adopté des stratégies spécifiques pour faire face à ce problème immédiat de ressources humaines. Plusieurs recrutent activement du personnel plus jeune ou ont mis en place un programme professionnel en faveur des débutants. D'autres reconnaissent que, en l'absence de possibilités d'évolution de carrière, les agents contractuels ne sont guère incités à rester durablement dans l'organisation.

Certains membres du CAD mettent l'accent sur l'égalité des chances dans la gestion des ressources humaines et prêtent une grande attention à la répartition entre hommes et femmes, ainsi qu'à la diversité ethnique et au recrutement de personnes ayant des besoins particuliers. Dans certains pays, les femmes sont presque aussi nombreuses que les hommes parmi les emplois de niveaux faible et intermédiaire, mais représentent une part moins élevée des postes de haut niveau. Ces questions d'égalité des chances relèvent habituellement des politiques générales du gouvernement en matière d'emploi, mais les pays membres prennent parfois des mesures spéciales pour promouvoir expressément l'égalité parmi le personnel chargé de la coopération pour le développement. L'emploi et la gestion des personnes séropositives ou souffrant du sida, autre aspect de la problématique de l'égalité des chances, sont traités plus loin.

\section{Sous-traitance}

Les pays membres du CAD peuvent choisir de compléter leurs capacités humaines par la sous-traitance d'activités comme le soutien technique, la recherche, l'évaluation ou la gestion d'initiatives spécifiques de développement. Pour une sous-traitance efficace, l'organisme d'aide doit répertorier les activités qui peuvent être confiées à l'extérieur et s'assurer que l'administration a la capacité de gérer les travaux du personnel sous contrat et des consultants. Les agents employés sous contrat de moyenne ou courte durée peuvent apporter des compétences spécialisées très précieuses sans faire partie du personnel permanent, et les contrats peuvent concerner des activités au siège ou sur le terrain. Parfois, le nombre d'agents sous contrat peut dépasser celui des agents permanents, en raison de la 
plus grande souplesse financière que permet le recrutement de personnel sous contrat ou de consultants dont les salaires sont payés au moyen de fonds versés aux programmes (qui, autrement, pourraient servir à financer des activités de développement).

Les organisations, agents et consultants employés sous contrat répondent à des besoins techniques et spécialisés, mais ne peuvent pas contribuer à la mémoire institutionnelle ni à une bonne exploitation des enseignements tirés de l'expérience. Lorsque les organismes d'aide préfèrent recruter des experts techniques sous contrat de courte ou de moyenne durée plutôt que du personnel permanent, l'absence de perspectives de carrière et de promotion peut être source de frustrations et se traduire par une forte rotation du personnel, préjudiciable elle aussi à la continuité des activités.

Le Service de la gestion publique de l'OCDE a établi une série de principes directeurs qui définissent les principaux facteurs permettant de tirer pleinement profit des avantages de la sous-traitance (voir encadré 7.1). Ceux-ci n'ont pas été conçus exclusivement pour les organismes d'aide, mais plusieurs facteurs présentent un intérêt particulier pour les gestionnaires de l'aide :

- Les services attendus doivent être définis en termes de produits ou de résultats et non d'intrants. Autrement dit, il convient de définir l'activité et non la façon dont elle doit être réalisée. Le sous-traitant doit avoir une marge de manœuvre suffisante pour pouvoir innover dans l'exécution des activités et ainsi obtenir des gains d'efficience. La définition de ces produits ou résultats doit être aussi complète que possible et comporter les indicateurs de qualité de service appropriés.

- La sous-traitance d'une activité ne diminue en rien la responsabilité de l'organisation en ce qui concerne son exécution. Cela vaut en particulier pour service fourni à une tierce partie.

- L'organisation doit suivre régulièrement et de façon formelle les performances du sous-traitant afin de s'assurer que les normes de performance fixées par contrat sont respectées. Lorsque les informations relatives aux résultats sont communiquées par le sous-traitant, leur exactitude doit être vérifiée.

\section{Encadré 7.1. Mieux sous-traiter les services publics : principes directeurs et études de cas}

À sa réunion de 1996, le Comité de la gestion publique de l'OCDE a approuvé une série de principes : Mieux sous-traiter les services publics : principes directeurs et études de cas. Ces principes directeurs ne visent pas à définir les activités qui devraient être sous-traitées, mais plutôt à identifier les méthodes qui permettent le mieux de déterminer s'il y a lieu de sous-traiter des services publics et la meilleure procédure à suivre une fois prise la décision.

Ils recensent sous huit rubriques les principaux facteurs qui permettent de tirer pleinement profit des avantages de la sous-traitance. Ce sont les suivants :

1. Assurer la participation du personnel de direction et favoriser la reconfiguration.

2. Mettre l'accent sur les questions relatives au personnel.

3. Définir les services attendus en termes de résultats.

4. Suivre les résultats et favoriser les relations de coopération.

5. Assurer la validité des comparaisons.

6. Développer et tenir à jour les compétences requises. 
- L'existence de marchés concurrentiels est essentielle pour que la sous-traitance puisse avoir des effets bénéfiques. L’État doit favoriser les marchés concurrentiels en reconnaissant que ses pratiques en matière de sous-traitance peuvent jouer un rôle déterminant dans le développement des marchés des services considérés.

- Les organisations qui confient des activités à des sous-traitants doivent tenir à jour leur connaissance du marché considéré ainsi que leur savoir technique concernant les activités en question. Cet élément est indispensable pour pouvoir communiquer d'égal à égal avec le fournisseur et être en mesure par la suite de lancer un nouvel appel d'offres. Cela vaut tout particulièrement dans le cas de la sous-traitance d'activités complexes.

\section{Mobilité du personnel et décentralisation}

Le renforcement du dialogue sur le terrain entre le gouvernement partenaire et les autres donneurs et la délégation accrue de responsabilités aux missions de terrain accentuent les besoins de spécialistes du développement expérimenté et qualifié à ce niveau. Dans la plupart des pays membres du CAD, le personnel du siège peut être envoyé sur le terrain et y acquérir un savoir et une expérience qu'il ramènera au siège ou exploitera dans un autre pays en développement lorsque son affectation prendra fin. Cependant, quelques-uns des plus centralisés n'appliquent pas ce système de rotation entre le personnel du siège et de terrain. La gestion du programme de coopération pour le développement est alors confiée à des diplomates en poste dans le pays, qui n'ont pas toujours les compétences de gestion nécessaires pour mettre en œuvre l'aide au développement dans les conditions d'isolement qui sont souvent celles des postes de terrain, ou à des agents recrutés localement sous contrat de durée déterminée. Aucune de ces deux solutions n'est favorable au renforcement des compétences en développement à l'intérieur du système d'aide du pays ni à une plus grande décentralisation des responsabilités au niveau local. L'absence de rotation limite par conséquent la capacité d'évolution des systèmes de coopération de ces pays dans la direction prise par de nombreux autres membres du CAD.

Parmi les organismes qui décentralisent la gestion du personnel et des responsabilités vers le terrain, les questions de ressources humaines apparaissent comme un défi de première importance. Il convient par exemple de se demander dans quelle mesure la décentralisation conduit à rationaliser les ressources humaines au siège. Si les responsabilités et la prise de décision sont décentralisées, certains niveaux administratifs existant au siège peuvent devenir inutiles, de sorte qu'une réorganisation du personnel devient nécessaire sous une forme ou sous une autre. Si, toutefois, les fonctions restent pour l'essentiel assumées par le siège, les tâches et les responsabilités risquent de se chevaucher. Il faut donc disposer d'un bon système de communication entre le siège et les agents en poste sur le terrain. Dans cette perspective, certains membres du CAD modernisent leurs équipements de communication, en se dotant notamment de matériel de vidéoconférence, solution qui peut cependant se révéler trop coûteuse pour d'autres membres dont les systèmes de communication doivent malgré tout être améliorés. Du fait de l'accroissement des effectifs du personnel de terrain, les pays partenaires risquent de devoir rencontrer et gérer de plus en plus de spécialistes des organismes d'aide dont le rôle et le mandat ne sont pas toujours clairs. Dans certains pays, tous les donneurs sont représentés par des spécialistes de domaines particuliers. L’harmonisation de l'aide devrait permettre une plus grande rationalisation des besoins de ressources humaines spécialisées entre les pays membres du CAD et à l'intérieur de chaque pays. 
Un exemple : le Japon

Le Japon renforce les activités de ses bureaux à l'étranger par une approche de "gestion axée sur le terrain ». La Banque japonaise pour la coopération internationale délègue davantage de pouvoirs à ses bureaux sur le terrain, en particulier pour la gestion des projets en cours ayant déjà fait l'objet d'un accord de prêt. Les bureaux locaux assument ainsi la responsabilité de la surveillance des procédures d'achat confiées pour l'essentiel aux pays partenaires et de l'approbation des sous-projets. L'Agence japonaise de coopération internationale a aussi renforcé sa capacité de soutien des pays partenaires en transférant davantage de personnel et de compétences à ses bureaux à l'étranger. Fin 2006, la JICA aura accru ses effectifs à l'étranger de près de 200 personnes, de sorte que le rapport entre le personnel du siège et le personnel de terrain sera de 1:1 (en excluant les agents, experts et volontaires locaux). En outre, la JICA met en place six « Bureaux d'assistance régionale » permettant de renforcer les activités pratiques et d'améliorer l'efficacité. Elle a aussi délégué davantage de compétences aux bureaux à l'étranger pour la définition, la planification, la mise en œuvre et l'évaluation des programmes ou projets.

\section{Ressources humaines sur le terrain}

\section{Effectifs présents au Mozambique}

Pour montrer la grande diversité des approches des pays membres en ce qui concerne les ressources humaines déployées sur le terrain, on a essayé de déterminer le nombre d'agents professionnels* utilisés par les pays membres du CAD pour gérer et mettre en œuvre leur programme d'aide au Mozambique. On trouve d'un côté de la fourchette des pays représentés par six agents ou moins et de l'autre deux pays ayant plus de 75 agents sur place (voir graphique 7.1). Bien que les effectifs moyens soient de 21 personnes, la plupart des pays sont représentés par une équipe comprise entre 10 et 15 agents. L'un des pays a 40 conseillers en poste au Mozambique. Plusieurs pays ont indiqué qu'ils avaient commencé de réduire progressivement leurs effectifs sur le terrain, dans le cadre d'une évolution plus générale privilégiant l'aide fournie au titre de programmes plutôt que de projets. Beaucoup de représentants des pays membres du CAD interrogés au Mozambique avaient l'impression que les effectifs présents sur le terrain ne permettaient pas de prendre pleinement part à l'ensemble des mécanismes de coordination des donneurs et de se tenir au courant des évolutions dans les principaux domaines d'aide. Quelques pays ont reconnu que, de ce fait, leur personnel passait trop de temps à participer à des réunions à Maputo et risquait de ne plus s'intéresser suffisamment à la situation des parties les plus isolées et les plus pauvres du pays.

Les effectifs des agents du siège chargés de soutenir le personnel en poste au Mozambique présentent aussi de grandes différences. Dans un cas, quatre agents du siège se consacrent uniquement au programme d'aide au Mozambique alors que, dans un autre, deux personnes seulement sont chargées de toutes les activités qui concernent l'Afrique. Dans la plupart des pays, le personnel affecté à l'aide au Mozambique est compris entre une personne à temps partiel et deux personnes à temps plein. Beaucoup de membres ont

\footnotetext{
* Sans inclure dans ce travail les catégories professionnelles suivantes: volontaires, assistants administratifs, secrétaires, chauffeurs, agents de sécurité, personnel de ménage et jardiniers.
} 


\section{Graphique 7.1. Importance du programme et effectifs professionnels au Mozambique}

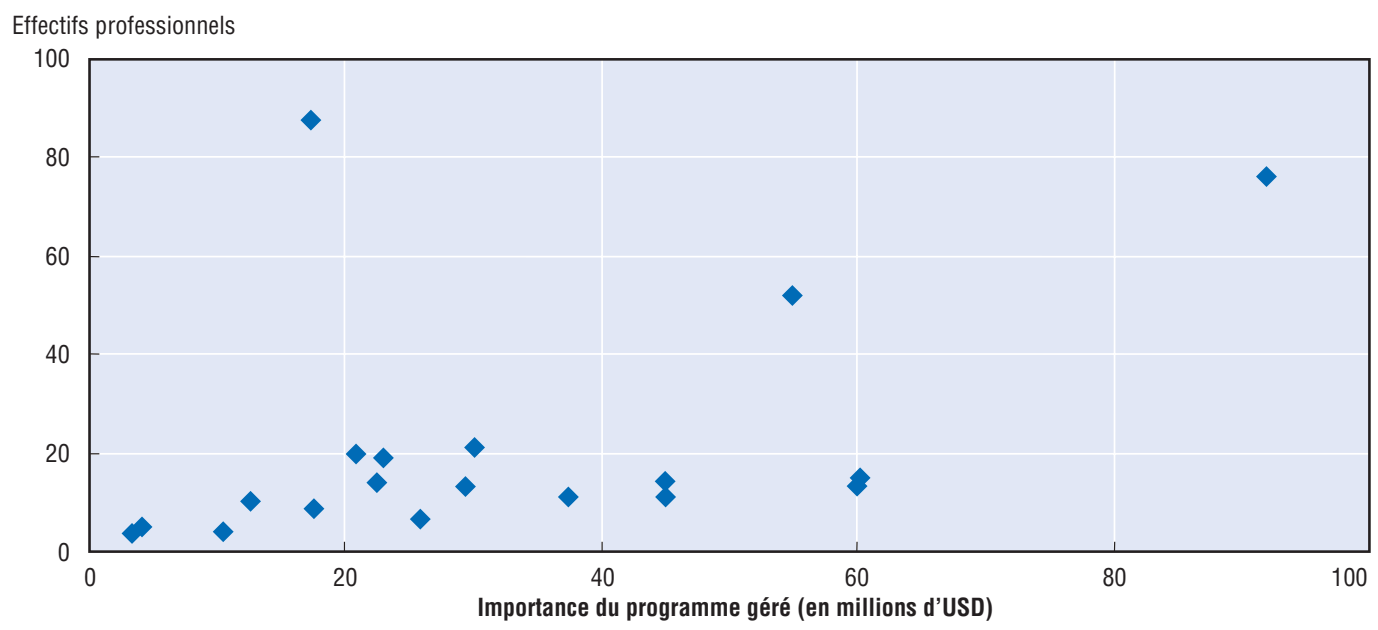

Source: Informations fournies par les pays membres du CAD.

indiqué que certains services du siège s'occupant d'aspects thématiques sont amenés à prendre part occasionnellement aux activités en faveur du Mozambique.

Le rapport entre l'importance du programme géré et le nombre d'agents sur le terrain (en tenant compte du personnel de soutien disponible au siège) fournit une base préliminaire de comparaison des pays membres du CAD. Dans neuf pays, quelle que soit l'importance du programme, un agent gère en moyenne de 0.7 million d'USD à 1.5 million d'USD par an (voir graphique 7.2). Dans quatre pays, un agent est responsable

Graphique 7.2. Montant géré par agent professionnel au Mozambique

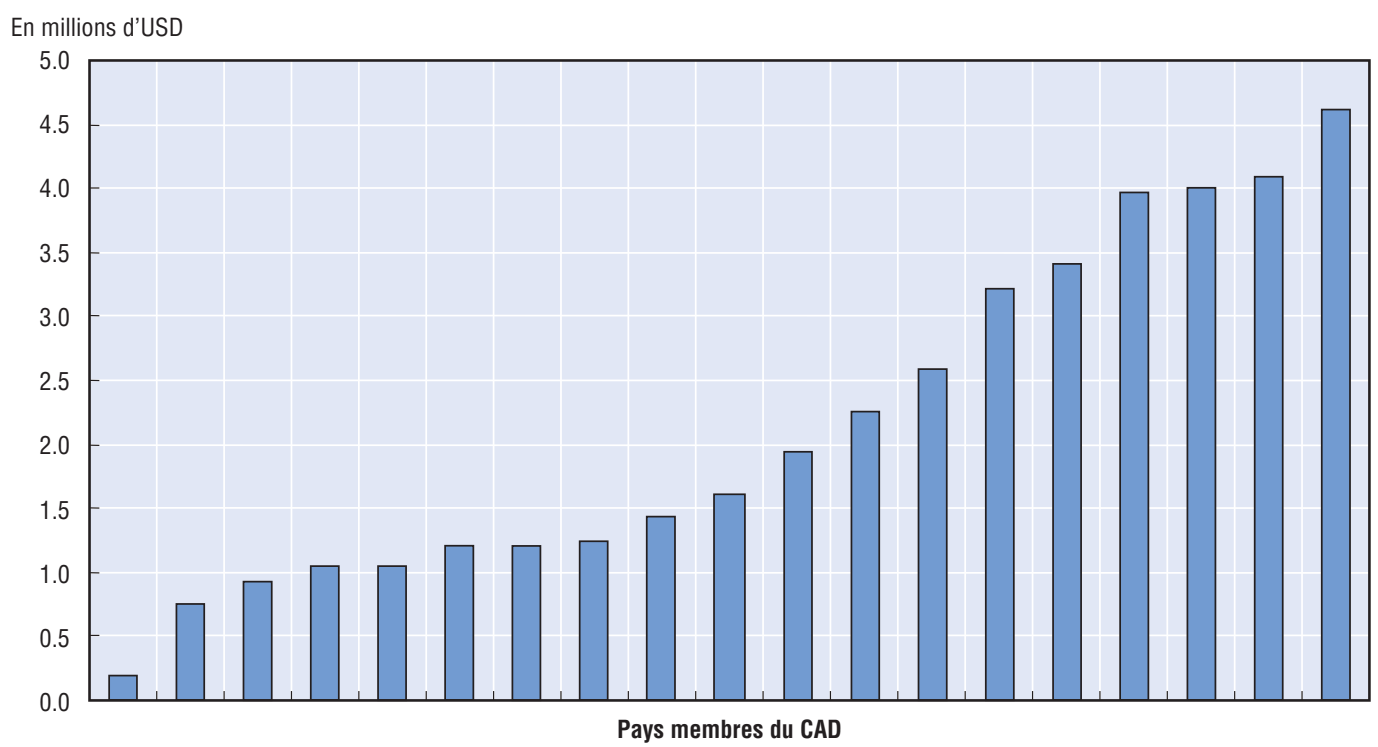

Source: Informations fournies par les membres du CAD. 
de plus de 4 millions d'USD par an, tandis qu'à l'autre extrémité de l'échelle, un agent gère 0.2 million d'USD. Le montant moyen géré par chaque agent des pays membres du CAD présents au Mozambique est de 1.5 million d'USD environ par an.

L'utilisation accrue de l'aide-programme parallèlement à d'autres formes d'aide peut avoir des conséquences sur les effectifs et les compétences des agents en poste dans les pays partenaires. Le personnel de terrain de certains membres du CAD indique qu'il faudrait disposer de meilleures compétences dans des domaines comme la communication entre les personnes, la négociation, la réflexion stratégique, l'analyse et la recherche. Les organismes d'aide ne s'accordent pas sur le fait que l'aide-programme exige des effectifs plus nombreux ou moins nombreux que d'autres formes d'aide, mais cette constatation même indique combien il est important de faire preuve, dans la gestion du personnel, de souplesse et de capacité d'adaptation à toute évolution de la situation.

\section{Recrutement de personnel local}

La plupart des membres du CAD reconnaissent que les agents recrutés sur place pour les bureaux locaux (ressortissants du pays partenaire, du pays donneur ou de pays tiers) apportent une valeur ajoutée aux missions de terrain, dans la mesure où ils sont souvent les bénéficiaires de la mémoire institutionnelle de la mission et possèdent des connaissances locales difficiles à acquérir pour le personnel international. Dans le cadre de leurs programmes de décentralisation, plusieurs pays cherchent notamment à renforcer le recrutement de personnel local pour occuper des postes professionnels, parfois de gestion, sur le terrain. Le recrutement d'experts techniques locaux présente des avantages considérables, mais on risque aussi de débaucher du personnel expérimenté employé dans les administrations ou les organisations locales. Même si beaucoup d'organismes cherchent à éviter ce problème, il leur est difficile en pratique d'empêcher le personnel local d'occuper les postes lucratifs qu'ils proposent. Certains d'entre eux ont adopté l'anglais comme langue de travail pour faciliter la communication entre le siège et le personnel employé localement, qui peut être de plusieurs nationalités.

$\mathrm{Au}$ Mozambique, la majorité des pays membres du CAD ont indiqué qu'ils n'avaient pas pris de mesures explicitement destinées à encourager une carrière de longue durée des agents locaux, mais ont signalé pour la plupart l'existence de formations, au Mozambique ou au siège. Deux pays ont cependant adopté des mesures dans ce sens, l'un d'eux offrant des contrats permanents ainsi qu'une formation complète, un régime de retraite et la possibilité d'être muté à d'autres postes de terrain. Plusieurs pays encouragent leur personnel local, explicitement ou non, à rechercher à terme d'autres emplois dans la société mozambicaine, tandis que d'autres doutent du réalisme d'une telle proposition, compte tenu de la baisse de salaire qui résulterait le plus souvent d'un tel changement de poste. Dans le secteur de la santé, les pays sont de plus en plus nombreux à avoir signé un protocole d'accord indiquant qu'ils éviteront de recruter des fonctionnaires locaux.

\section{La question du VIH/sida}

De nombreux organismes d'aide, en particulier ceux qui emploient beaucoup de personnel en Afrique subsaharienne, doivent faire face à un grave problème de ressources humaines, celui de l'incidence effective et potentielle du VIH/sida sur leurs effectifs. Certains pays ont adopté des politiques concernant le VIH/sida sur le lieu de travail, pour le personnel du siège et le personnel recruté localement (voir ci-dessous), ou sont sur le point de le faire. Au Mozambique, certains organismes ont mis en place des politiques 
locales qui prévoient des actions de sensibilisation, des tests et des traitements gratuits et confidentiels, même si la prise en charge du traitement des membres de la famille n'apparaît pas toujours suffisante à l'heure actuelle. Parmi ceux qui n'ont pas adopté de politiques officielles pour le lieu de travail, certains disposent néanmoins de régimes généraux d'assurance médicale couvrant certains aspects du traitement. Certains pays considèrent que leur personnel local est trop peu nombreux pour qu'une politique spécifique soit mise en place, et qu'ils peuvent examiner ces situations au cas par cas, comme ils l'ont parfois déjà fait.

\section{Un exemple : l'Allemagne}

À partir de la politique adoptée pour l'ensemble de la GTZ sur le VIH/sida (conformément aux recommandations de l'Organisation internationale du travail), un ensemble de principes directeurs a été mis en place pour application dans le cadre du programme de la GTZ au Mozambique. Ces principes directeurs concernent le personnel local et portent sur la création d'un environnement propice au travail et la non-discrimination. Le personnel local reçoit régulièrement une formation actualisée et des informations sur la prévention du VIH/sida, adaptée en fonction de l'âge et du sexe de chacun. Un conseiller désigné pour chaque projet organise des sessions d'information et de formation sur différents aspects du VIH/sida. Des matériels d'information et de référence sont mis à la disposition du personnel et il est possible de faire appel à une ONG locale pour des consultations plus approfondies. La GTZ finance la prophylaxie et le traitement post-exposition pour l'agent, son compagnon (à condition qu'ils vivent ensemble depuis un an au moins) et ses enfants jusqu'à l'âge de 18 ans. La couverture assurée concerne aussi le traitement des infections opportunistes et la thérapie antirétrovirale, à condition qu'ils soient prescrits par un médecin reconnu. Si un agent ne peut plus exercer ses fonctions du fait de la maladie, la GTZ prend en charge les frais de recyclage professionnel et l'affecte à un autre poste dans le cadre du projet. La prise en charge de congés maladie de longue durée est également prévue, ainsi que le versement d'une indemnité au plus proche parent en cas de décès. 



\section{Chapitre 8}

\section{Mise en œuvre de programmes bilatéraux dans les différents contextes des pays partenaires}

Les possibilités de lutte contre la pauvreté ainsi que les efforts déployés dans ce domaine varient selon chaque pays en développement et évoluent constamment. Les pays membres du CAD doivent par conséquent adapter la stratégie de mise en cuure de leurs programmes d'aide extérieure en fonction du contexte spécifique de chaque pays partenaire et opérer des ajustements selon le contexte social et politique local, le dynamisme et les capacités des institutions nationales, le degré et l'ampleur de la pauvreté, ainsi que ses caractéristiques spatiales et géographiques. Si la mise en cuvre des programmes bilatéraux se prête difficilement à des généralisations en raison de la diversité des situations des pays en développement, il est maintenant largement admis qu'il convient d'adopter une approche fondée sur le partenariat dans les pays qui présentent un environnement suffisamment propice pour promouvoir le développement. Dans cette perspective, les pays membres s'attachent avec succès à renforcer l'harmonisation et l'alignement de leurs programmes de développement, même si leur tâche est loin d'être déterminée. Ainsi, il reste encore à de nombreux pays à définir des stratégies qui fournissent une meilleure prévisibilité à leurs partenaires quant aux niveaux d'aide prévus. Dans d'autres circonstances, notamment dans les situations de "partenariat difficile", différents types de stratégies seront nécessaires et les organismes d'aide au développement seront peut-être amenés à adapter leur approche pour tenir compte de l'évolution du pays concerné. 


\section{Mise en œuvre des partenariats pour le développement}

\section{Recommandations pratiques pour un bon fonctionnement}

Dans les pays partenaires affichant une situation saine sur le plan macro-économique et dotés d'institutions opérationnelles, l'aide étrangère peut être apportée efficacement dans le cadre de partenariats qui mettent l'accent sur les droits et les devoirs tant des partenaires extérieurs que des pays en développement. Au cours des dernières années, de nombreux travaux ont été entrepris pour tenter de mieux comprendre les principes sur lesquels s'appuient les partenariats pour le développement et pour formuler des recommandations pratiques pour un bon fonctionnement des partenariats au service de la lutte contre la pauvreté (voir encadré 8.1).

\section{Alignement, harmonisation et rationalisation}

L'utilisation des stratégies nationales de lutte contre la pauvreté comme cadre général de la coopération pour le développement est un objectif primordial pour les nombreux pays membres du CAD qui reconnaissent la pertinence de l'approche des Cadres stratégiques de lutte contre la pauvreté et qui estiment que ces stratégies constituent des bases solides à partir desquelles renforcer les partenariats et améliorer la coordination entre les donneurs. Une étude réalisée conjointement par le FMI et la Banque mondiale ${ }^{1}$ tend cependant à démontrer que si les organismes d'aide au développement acceptent, sur le plan des principes, d'aligner le contenu de leurs programmes sur les priorités énoncées dans les Cadres stratégiques de lutte contre la pauvreté, nombre d'entre eux n'ont pas dépassé le stade du vœu pieux. L'absence d'établissement de priorités et le manque de précision de nombreux Cadres stratégiques de lutte contre la pauvreté rend l'alignement difficile, même si l'on observe des améliorations aussi bien dans les processus CSLP que dans leur contenu, depuis que les pays partenaires ont commencé à les élaborer et à les mettre en œuvre. Cette étude révèle que le processus CSLP est principalement parvenu, à ce jour, à rendre les gouvernements et les sociétés civiles partenaires plus responsables de leurs stratégies de lutte contre la pauvreté, à instaurer un dialogue plus ouvert au sein du gouvernement et avec certaines composantes de la société civile et à faire progresser la place de la lutte contre la pauvreté dans les débats de fond.

En 2003, les membres du CAD sont convenus, dans la Déclaration de Rome sur l'harmonisation, d'harmoniser les systèmes des pays partenaires et - par conséquent - de s'aligner les uns sur les autres. Toutefois, l'alignement avec les systèmes et les procédures des pays partenaires peut s'avérer encore plus problématique pour les pays membres du CAD que l'alignement avec les politiques et priorités en matière de développement des pays partenaires. Cependant, la situation progresse et toute une série d'activités d'harmonisation est en cours au niveau de divers pays partenaires, y compris la formation de groupes de travail pouvoirs publics/donneurs, l'élaboration de programmes d'action conjoints entre les donneurs et les pouvoirs publics portant sur l'harmonisation et l'alignement et la conception de stratégies d'aide conjointes entre plusieurs donneurs. 


\section{Encadré 8.1. Recommandations pratiques pour un bon fonctionnement des partenariats au service de la lutte contre la pauvreté}

Travailler en partenariat suppose que les organismes d'aide au développement changent radicalement leurs modes de relation entre eux et avec les pouvoirs publics et la société civile des pays partenaires. Cela implique parfois aussi qu'ils modifient profondément leur manière de travailler dans les pays partenaires. Les lignes directrices $d u C A D:$ La réduction de la pauvreté formulent des recommandations pratiques sur la façon de travailler en partenariat :

- Prendre comme cadre général de la coopération pour le développement la stratégie de lutte contre la pauvreté et le budget national du pays partenaire.

- Reconnaître le leadership du pays partenaire.

- Définir clairement les rôles et responsabilités des différents acteurs (gouvernement, organismes bilatéraux, institutions financières internationales et régionales, organismes des Nations unies, société civile, syndicats, secteur privé).

- Ne jamais travailler seul. Avant d'entamer une discussion ou une action, rechercher d'autres partenaires (dans la communauté de l'aide au développement, le gouvernement ou la société civile) susceptibles d'y participer.

- Investir dans des mécanismes de coordination (qui doivent permettre, sous la direction du pays, de coordonner la planification stratégique ex ante et la mise en œuvre conjointe), et définir de façon précise les modalités, le moment et le lieu des échanges avec les autres partenaires extérieurs et locaux.

- Promouvoir et harmoniser les travaux communs (collectes de données, analyses, missions évaluations, gestion et comptabilité des flux d'aide) et partager l'information (données, analyses, actions et programmes envisagés) avec les autres partenaires.

- Simplifier et rationaliser, dans la mesure du possible, les exigences administratives et financières de l'organisme d'aide au développement (par exemple en ce qui concerne la gestion financière et la comptabilité, les phases préparatoires de déroulement du projet, les comptes rendus et les exercices de suivi) et renforcer les systèmes mis en place par l'administration du pays partenaire dans ces mêmes domaines.

- Faciliter la mobilisation, la participation, la surveillance et l'évaluation locales.

- Assurer le renforcement des capacités afin d'affermir la prise en main par le gouvernement de la coordination de la lutte contre la pauvreté et des processus de consultation, et de permettre à la société civile, y compris aux organisations féminines et à celles qui militent en faveur de l'égalité des sexes, de participer pleinement au processus de consultation et de suivre activement et d'évaluer les politiques et programmes de lutte contre la pauvreté - sans pour autant porter préjudice à l'autorité du gouvernement partenaire ou aux institutions démocratiques nationales.

Les donneurs peuvent également travailler en collaboration pour simplifier ou rationaliser leurs procédures et pratiques administratives et alléger ainsi la charge supportée par les gouvernements partenaires. Parmi les autres recommandations pratiques (voir encadré 8.2), l'organisation de réunions conjointes à haut niveau et de groupes consultatifs conjoints est susceptible, entre autres, de réduire la charge de travail liée à la gestion de l'aide étrangère. Même si la tenue de réunions bilatérales à haut niveau se poursuit au Mozambique, la tendance à l'heure actuelle semble être plutôt d'organiser des consultations au coup par coup, en cas de besoin, plutôt que tous les ans. En outre, les 


\section{Encadré 8.2. Recommandations pratiques pour de bonnes relations entre donneurs}

Des recommandations pratiques à l'attention des organismes d'aide au développement ont été formulées dans Les lignes directrices et ouvrages de référence du CAD : Harmoniser l'aide pour renforcer son efficacité. Parmi elles figurent notamment :

- Consulter les gouvernements partenaires pour rechercher les moyens de réduire la charge administrative par l'instauration de dispositifs de collaboration.

- Veiller à la cohérence des communications avec les gouvernements partenaires en réduisant les divergences de vues sur les principes d'action au minimum requis afin de permettre au gouvernement partenaire de faire des choix éclairés. Les donneurs et les gouvernements partenaires peuvent améliorer la cohérence en désignant un chef de file pour représenter l'ensemble des donneurs qui interviennent dans un secteur ou sur une question transversale.

- Partager l'information relative aux activités des donneurs dans un secteur avec les autres donneurs et le gouvernement partenaire.

- Normaliser les systèmes et simplifier les procédures en utilisant, lorsque cela est possible, les systèmes des pays partenaires.

- Élaborer des procédures communes en consultation avec le gouvernement partenaire, afin de permettre une participation plus active des pays partenaires et l'élaboration conjointe de systèmes communs aux pays partenaires et aux donneurs.

- Partager des exemples de procédures communes mises en place dans tel ou tel pays, afin de pouvoir dégager des enseignements susceptibles d'être appliqués plus largement.

mécanismes de mise en commun des fonds attirent un nombre croissant de donneurs et des montants de plus en plus importants d'APD, ce qui rend de plus en plus nécessaires des évaluations conjointes.

Au-delà du renforcement de la coopération et de la rationalisation de leurs procédures, les organismes d'aide au développement peuvent s'engager dans une collaboration plus formelle dans laquelle un donneur " chef de file " agira au nom d'un ou de plusieurs autres donneurs "délégants". Le donneur déléguant, qui peut également être qualifié de " partenaire muet » renonce à une certaine proportion de son identité propre. Une visite au Mozambique a toutefois démontré que si des représentants sur le terrain de certains pays membres du CAD étaient disposés à adopter la pratique des partenariats muets, leurs sièges n'étaient pas encore prêts à approuver ces initiatives. L'intensité et le type de coopération par délégation peuvent varier considérablement: le chef de file peut agir au nom des autres donneurs lors du lancement initial d'une activité ou tout au long du cycle d'un projet ou d'un programme. Ainsi au Bangladesh, six organismes d'aide au développement bilatéraux, associés à cinq autres donneurs et à la Banque de développement asiatique (AsDB) - l'organisme chef de file - financent conjointement un programme de développement du secteur de l'enseignement primaire, et rendent compte chaque trimestre auprès des donneurs. Au Mozambique et en Zambie, les pays membres du CAD déploient des efforts considérables pour rattraper le retard en matière d'alignement, d'harmonisation et de rationalisation des priorités et des systèmes du gouvernement (voir encadré 8.3). 


\section{Encadré 8.3. La coopération entre donneurs au Mozambique et en Zambie}

A l'heure actuelle, le gouvernement du Mozambique reçoit un appui budgétaire direct de 15 bailleurs de fonds, le " G 15 », suite à la signature d'un Mémoire d'entente entre ces derniers et le gouvernement national. Le $\mathrm{G} 15$ et le gouvernement se réunissent tous les trimestres pour examiner les priorités de recettes et de dépenses, l'exécution du budget et l'avancement de la mise en œuvre du PARPA (la stratégie nationale de lutte contre la pauvreté). Une évaluation du programme a lieu chaque année en mars/avril ; elle débouche sur des engagements provisoires de soutien budgétaire pour l'année civile suivante. Le versement des fonds est régi par un dispositif commun, simple, qui assure un financement non lié et non assorti de conditions quant à son utilisation. Le gouvernement du Mozambique doit mettre en œuvre le programme de réduction de la pauvreté défini dans le PARPA et le cadre budgétaire à moyen terme, et se conformer au programme macro-économique arrêté avec le FMI au titre du Fonds pour la réduction de la pauvreté et la croissance.

En Zambie, sept donneurs ont lancé le programme d'harmonisation - le Danemark, la Finlande, l'Irlande, les Pays-Bas, la Norvège, la Suède et le Royaume-Uni - peu de temps après le Forum de haut niveau sur l'harmonisation qui a eu lieu à Rome. En mars 2003, le gouvernement de la République de Zambie a rédigé un accord avec des donneurs intéressés portant sur la poursuite de l'harmonisation en Zambie. Né de leurs travaux, le Cadre pratique d'harmonisation s'inspire à la fois de la Déclaration de Rome sur l'harmonisation, des travaux du CAD, du Programme spécial d'assistance pour l'Afrique ainsi que des enseignements tirés d'expériences concluantes en matière d'harmonisation dans les secteurs de la santé et de l'enseignement en Zambie. Sous l'impulsion du gouvernement zambien, des institutions financières internationales, la Commission européenne et d'autres donneurs bilatéraux travaillent actuellement à étendre l'harmonisation au-delà du groupe initial composé des sept membres. La stratégie globale sera à l'avenir régie par les principes suivants, auxquels souscrivent aussi bien le gouvernement que les donneurs :

- Leadership, coordination et orientation du gouvernement partenaire.

- Attachement à la réforme de la fonction publique.

- Réforme de la gestion des finances publiques.

- Engagement à utiliser le CSLP comme base de la planification et de l'évaluation stratégiques.

- Engagement à adopter des approches sectorielles et évolution possible vers un appui budgétaire direct.

La Zambie fournit également quelques exemples de coopération par délégation et de partenariats muets. La Norvège apporte une aide au secteur agricole national dans le cadre d'un partenariat muet avec les Pays-Bas. Le Royaume-Uni apportera une aide au programme d'approches sectorielles dans l'enseignement mené par l'ACDI. L'Irlande, les Pays-Bas, la Norvège, la Suède et le Royaume-Uni envisagent également de soutenir le Conseil national de lutte contre le sida de la Zambie; la Norvège assumerait les responsabilités en matière de gestion pour le compte des autres donneurs.

Source : www.aidharmonization.org. 
Afin de faciliter les partenariats pour le développement, l'alignement, l'harmonisation et la rationalisation, certains pays membres du CAD envisagent de délocaliser davantage la main-d'œuvre et les compétences sur le terrain, de façon à mieux appréhender les conditions locales, à réagir plus efficacement et à renforcer le dialogue. Les connaissances et l'expertise du personnel de terrain sont capitales pour prendre des décisions éclairées en matière de développement des programmes et permettent de nouer des interactions efficaces avec les interlocuteurs. La gestion des ressources humaines et les politiques en matière de recrutement doivent tenir compte de cet état de fait.

\section{Gestion des projets de développement}

Comme nous l'avons souligné précédemment, le financement des projets de développement reste un instrument d'aide important pour de nombreux pays membres du CAD, même si la coopération pour le développement privilégie de plus en plus les approches fondées sur les programmes. De nombreux pays, même parmi ceux qui optent pour ce dernier type d'approche, souhaitent continuer à soutenir des activités (projets) à l'échelon local, de façon à pouvoir évaluer les retombées des actions prises par les pouvoirs publics et de prendre en compte les enseignements ainsi tirés dans le dialogue mené au niveau national sur les politiques à suivre.

On remarque toutefois une désaffection considérable à l'égard des projets isolés, au profit des projets qui s'inscrivent dans un cadre de développement plus vaste, qui prend en compte les divers problèmes des populations démunies et renforce leurs capacités à acquérir des moyens d'existence durables. La gestion des projets doit en outre donner un rôle plus prépondérant aux pays partenaires, les responsabiliser davantage et contribuer aux objectifs d'harmonisation et d'alignement. Ainsi au Mozambique, plusieurs pays qui travaillent principalement par le biais de projets s'efforcent résolument d'accroître les retombées de leur action en se concentrant sur un nombre plus restreint d'activités de fond. Cette orientation permettra également de réduire, pour le Mozambique, les coûts de transaction inhérents à la gestion d'une multitude d'activités de développement ${ }^{2}$.

Les stratégies de gestion du cycle des projets sont désormais des pratiques courantes parmi les pays membres du CAD. Le processus de gestion du cycle des projets débute par la conception initiale d'une action de développement et se termine par une évaluation post-réalisation. Il s'applique à la fois aux projets et aux programmes et est principalement axé sur les bénéficiaires potentiels, l'évaluation détaillée et la mise en application d'un cadre d'action logique. Cette méthode fournit une approche structurée et logique en matière d'établissement des priorités et de définition des résultats escomptés d'une activité (l'annexe A.5 décrit en détail la stratégie de gestion du cycle des projets).

\section{Stratégies par pays}

La visite sur le terrain au Mozambique a révélé que la plupart des pays membres du CAD élaborent des stratégies par pays ou des documents d'action détaillés exposant le contexte, la justification, les objectifs et la stratégie de leurs programmes dans les principaux pays partenaires. Ces informations peuvent représenter un cadre théorique et pratique pour l'élaboration et la mise en œuvre des programmes de coopération menés en collaboration avec les gouvernements partenaires. Elles peuvent également permettre une articulation claire entre les besoins d'un pays partenaire et la manière dont le programme d'un organisme d'aide au développement peut contribuer à réduire la pauvreté à l'échelle nationale et à atteindre des objectifs dans ce domaine. Les priorités et les grandes 
orientations définies dans les stratégies par pays doivent s'appliquer à l'ensemble des organismes et ministères impliqués dans la mise en œuvre des différents aspects d'un programme national de coopération pour le développement.

Le travail en partenariat s'est traduit par un processus de plus en plus participatif d'élaboration des stratégies par pays et une consultation plus étroite des représentants des pouvoirs publics et des acteurs de la société civile (voir ci-dessous). Les partenariats supposent aussi que des engagements budgétaires fermes soient pris pour la durée de la stratégie, de sorte que les gouvernements partenaires puissent prévoir en conséquence et investir dans l'avenir, au lieu de répondre à des priorités sur le court terme. Il n'en demeure pas moins qu'au Mozambique, bien que les donneurs aient tous déclaré que leur stratégie nationale était conforme au PARAP (le plan national d'action pour la réduction de la pauvreté qui couvre la période allant de 2001 à 2005), la durée des stratégies nationales s'échelonne de deux à six ans et dépend pour l'essentiel des propres processus internes des donneurs. Cela ne facilite pas la gestion de l'aide étrangère du point de vue du pays partenaire.

\section{Un exemple : le Danemark}

La première stratégie de coopération avec le Mozambique élaborée par le Danemark couvrait la période allant de 1995 à 1999. Le pays a divulgué sa deuxième stratégie, qui porte sur les quatre années suivantes, en mai 2000. Rédigée en concertation avec les autorités du Mozambique, elle fait une large place aux données concrètes recueillies lors de stratégies de coopération antérieures, à l'évolution du pays partenaire et à la situation qui prévaut au Mozambique. Elle couvre les domaines suivants :

- La situation sur les plans politique et économique.

- La stratégie et les priorités du gouvernement du Mozambique en matière de développement.

- La stratégie de lutte contre la pauvreté du gouvernement du Mozambique.

- L’ampleur et la répartition des activités des donneurs.

- La coordination entre les donneurs.

- La coopération avec les ONG.

- L'état d'avancement du dialogue avec les autorités publiques.

- Les objectifs globaux du programme danois de coopération pour le développement.

- La stratégie de lutte contre la pauvreté du programme bilatéral.

- Les centres d'intérêt du programme.

- La mise en œuvre de la concentration sectorielle.

- Les thèmes transversaux.

- La coopération avec les entreprises, l'industrie, les instituts de recherche, les ONG et les consultants danois.

- Les difficultés de suivi et de contrôle liés aux efforts de coopération du Danemark.

- Les ressources humaines au Mozambique. 
- L'évaluation.

- Les principaux indicateurs sociaux et économiques.

- Les chiffres indicatifs de planification pour la période 2000-2004.

En théorie, et conformément au modèle de partenariat, les organismes d'aide au développement doivent prendre de plus en plus comme cadre général de coopération pour le développement la stratégie de lutte contre la pauvreté du pays partenaire. Toutefois, dans certains pays membres du CAD, les réalités politiques imposent l'élaboration de stratégies par pays sous une forme ou sous une autre entretiennent déjà des relations particulières avec des pays spécifiques. Dans ce cas, à mesure que les bilans de pauvreté et la qualité des CSLP s'améliorent, les stratégies nationales peuvent servir de trame à des stratégies par pays plus concises mais répondant aux exigences des pays membres du CAD.

\section{Appropriation au niveau local}

Dans le passé, les gouvernements de nombreux pays en développement ne disposaient pas des capacités suffisantes pour coordonner l'action des organismes d'aide au développement et veiller à ce qu'ils respectent les principes de partenariat. Cette situation est en train d'évoluer, d'une part parce que les gouvernements partenaires sont davantage conscients de leur leadership et de leur appropriation, et d'autre part en raison des initiatives en faveur du renforcement des capacités, soutenues par les donneurs. Le Nouveau partenariat pour le développement de l'Afrique (NEPAD) est une initiative importante financée par des capitaux africains et dirigée par des responsables africains, destinée à donner à l'Afrique les moyens de relever les enjeux auxquels elle est confrontée dans le domaine du développement. L'Inde est un exemple typique de pays en développement qui fait preuve de leadership et élabore des lignes directrices à l'attention des donneurs. Ce pays a récemment décidé de limiter ses sources d'aide extérieure d'État à État à six donneurs (voir ci-dessous).

\section{Un exemple : le NEPAD}

Le Nouveau partenariat pour le développement de l'Afrique est un cadre d'action stratégique pour le renouveau de l'Afrique piloté par des dirigeants africains. La finalité de ce programme de l'Union africaine est de s'attaquer aux problèmes graves que rencontre le continent africain, notamment l'escalade de la pauvreté et le sous-développement. Ses principaux objectifs consistent à éradiquer la pauvreté, à placer les pays africains, aussi bien sur le plan individuel que collectif, sur la voie d'une croissance et d'un développement durables, à mettre un terme à l'exclusion de l'Afrique du processus de mondialisation et à veiller à ce qu'elle soit pleinement et utilement intégrée à l'économie mondiale, et à améliorer la participation des femmes à la vie publique et économique. Les principes du NEPAD sont les suivants : bonne gouvernance ; appropriation et leadership de l'Afrique, participation vaste et profonde de l'ensemble des secteurs de la société ; ancrage du développement de l'Afrique dans ses ressources et les capacités de ses populations; partenariats entre les peuples africains; accélération de l'intégration aux niveaux de la région et du continent; développement de la compétitivité des pays et du continent africains ; établissement d'un nouveau partenariat mondial qui mette fin aux relations inégales entre l'Afrique et le monde développé ; et engagement à lier tous les partenariats avec le NEPAD aux 
Objectifs du millénaire pour le développement et aux autres objectifs et buts fixés en matière de développement.

\section{Un exemple : L'Inde}

En juin 2003, les autorités indiennes ont fait part de leur intention de limiter la dépendance du pays à l'aide étrangère en réduisant le nombre des donneurs à six : l'Allemagne, la Commission européenne, les États-Unis, la Fédération de Russie, le Japon et le Royaume-Uni. La contribution des vingt-deux autres donneurs, y compris des pays dont l'aide financière allouée à l'Inde représentait une proportion considérable de la totalité de leur programme d'aide étrangère, prendra fin à l'achèvement des programmes en cours. L'Inde a également annoncé qu'elle n'accepterait aucune aide liée à la fourniture de biens et de services par le donneur. En outre, le ministère des Finances a rendu publiques les dispositions relatives à l'élaboration de nouvelles lignes directrices destinées à régir, à l'avenir, les apports d'aide bilatérale en direction de l'Inde, et qui prévoient, notamment, des réunions annuelles avec les donneurs au début de chaque année budgétaire pour organiser l'aide.

\section{Soutenir le développement dans les situations de partenariat difficile}

Les situations de " partenariat difficile " se rencontrent dans les pays en développement où les objectifs en matière de développement n'ont que peu d'importance pour les gouvernements, comparés à d'autres objectifs tels que la volonté de rester au pouvoir ou d'accumuler des richesses. La répression politique, la corruption, les conflits violents et les violations des droits de la personne humaine comptent parmi les caractéristiques de ces régimes réfractaires. En l'occurrence, ces gouvernements partenaires ne s'engagent pas de façon crédible à adopter et mettre en œuvre des politiques efficaces, et la définition d'objectifs communs en matière de développement avec les donneurs potentiels fait défaut.

On peut établir une distinction importante entre les partenariats difficiles et les pays dans lesquels le gouvernement s'efforce de mettre en œuvre des politiques efficaces, mais ne dispose que de très faibles moyens pour y parvenir. Il convient peut-être alors d'adapter le modèle du partenariat aux "États fragiles " ou à ceux qui sortent d'un conflit ou sont menacés d'instabilité. Dans ces cas-là, l'aide étrangère peut jouer un rôle fondamental dans le renforcement des institutions, l'amélioration de la gouvernance et de la responsabilité et la promotion de la stabilité ; elle peut être en mesure, à terme, d'empêcher ces pays de basculer et de devenir des partenaires difficiles.

Le modèle du partenariat et la stratégie de développement arrêtés en commun ne peuvent plus s'appliquer aux situations de partenariat difficile. Toutefois, les pays du CAD admettent de plus en plus qu'un retrait total de la communauté des donneurs des pays où les partenariats sont difficiles aurait des répercussions sur les 500 millions de personnes, démunies pour la plupart, qui vivent dans ces pays, ainsi que sur les populations des pays voisins et la communauté internationale. Parmi ces répercussions figurent le risque d'une faillite totale de l'État, l'émergence de conflits, la déstabilisation de la région, l'intensification des souffrances et du dénuement des populations pauvres, sans compter de très faibles chances de redressement ${ }^{3}$. 
Compte tenu des efforts déployés par la communauté internationale pour promouvoir la stabilité à l'échelle mondiale, le CAD s'est récemment efforcé, dans certaines de ses initiatives, d'étudier les différentes stratégies de participation opportunes pour les pays membres du CAD qui interviennent dans des pays présentant une situation de partenariat difficile. Il n'existe pas de modèle unique applicable à l'ensemble des pays regroupés sous la catégorie de « partenariat difficile ». Les caractéristiques de ces pays varient considérablement et les organismes d'aide au développement doivent analyser conjointement la situation sociale et politique avec la plus grande attention, de façon à mieux comprendre quel type de partenariat est le plus adapté et de s'entendre sur sa nature.

L'expérience de certains pays membres du CAD montre qu'il est possible de continuer à agir dans les pays présentant une situation de partenariat difficile (voir encadré 8.4 à titre d'illustration). Le CAD a exposé quelques-unes des principales stratégies en matière de coopération pour le développement à mettre en œuvre dans ces contextes :

- Promouvoir un changement favorable aux populations pauvres : Il est possible de soutenir les institutions démocratiques et d'œuvrer en faveur de la mise en place d'un environnement propice à la démocratie dans les situations de partenariat difficile. La stratégie à cette fin est davantage orientée sur les principaux acteurs de changement à l'intérieur et à l'extérieur de l'État, de façon à relayer les idées nouvelles, à promouvoir le débat et à faciliter (ou accélérer) le changement. Les actions en faveur du renforcement des capacités de la société civile, en raison de son rôle en matière de sensibilisation de l'opinion publique, de même que le rôle du secteur privé, peuvent également être fondamentaux. Des mesures à moyen terme, telles que la formation des futurs dirigeants et l'éducation des filles, peuvent également être incluses.

- Maintenir les activités de développement en faveur des populations démunies: L'ensemble des pays reconnaissent la nécessité de garantir l'accès des populations démunies aux services, quelles que soient les difficultés rencontrées dans certains partenariats. La Banque mondiale propose de créer des Autorités indépendantes de services pour passer des contrats avec des agents prestataires de services. Les gouvernements hôtes ne seront peut-être toutefois pas prêts à accepter ces organismes et certains signes tendent à montrer que ces derniers risquent, avec le temps, de perdre leur indépendance. De nombreux pays membres du CAD préfèrent éviter de soutenir durablement des services parallèles. Les organismes d'aide au développement peuvent financer des activités du secteur privé, d'ONG internationales et nationales et de groupes religieux qui remplissent souvent une mission importante dans la fourniture des services dans de tels pays en raison des défaillances du secteur public. Les organismes d'aide au développement peuvent également envisager de mener leurs activités en ayant recours aux structures gouvernementales infranationales ou locales, de façon à s'assurer la participation du gouvernement et à maintenir le dialogue.

- Adapter la coordination entre les donneurs et renforcer la cohérence de l'action publique : Cette exigence est d'autant plus impérieuse que le partenariat est difficile, même si les donneurs ne poursuivent pas tous leur engagement. Le manque de coordination peut avoir de graves répercussions dans un contexte où les ressources extérieures sont limitées, les points d'ancrage rares et les besoins considérables. Les stratégies en matière de coordination pourraient prévoir la désignation d'organismes d'aide au développement qui feront office de référents pour certaines tâches, comme l'analyse, le soutien au secteur privé, le soutien au renforcement des secteurs ou au système politique. Le ciblage de la totalité de l'aide 
étrangère sur un nombre limité d'activités peut être plus indiqué qu'une division du travail. Une coordination efficace passe par:

* L'instauration de critères d'évaluation communs.

* Une meilleure mise en commun des analyses.

* Un accord sur les conditions de participation.

* La désignation d'organismes pilotes ou de référence.

* La mise à profit des avantages comparatifs des organismes bilatéraux et multilatéraux.

\section{Encadré 8.4. Cadre de développement national mis en place par l'ACDI en Haïti}

Face aux conflits et aux crises qui ont marqué l'histoire d'Haïti et aux faibles résultats du pays, l'ACDI a décidé en 2003 que la situation d'Haïti en matière de développement nécessitait une approche fondée sur le modèle conçu par le CAD pour les situations de partenariat difficile. L'ACDI a par conséquent élaboré une orientation stratégique d'engagement à long terme avec Haïti qui supposerait de dégager les ressources nécessaires pour soutenir le développement, de limiter les répercussions des échecs rencontrés et de créer des mesures d'incitation destinées à faire participer, le plus largement possible, les groupes les plus désavantagés de la population aux processus d'évolution. Les éléments principaux de cette approche sont les suivants :

- La prise en charge locale : La prise en charge doit se construire graduellement d'abord en établissant des partenariats avec des interlocuteurs locaux qui ont déjà amorcé une prise en charge de leur développement, et en favorisant le développement d'une masse critique d'interlocuteurs engagés dans des actions de développement et capables d'apporter des solutions aux problèmes du pays.

- L'amélioration de la coordination entre donneurs: Dans le cas d'Haïti, cette coordination ne peut venir ni des pouvoirs publics, ni d'organisations de la société civile à court et même à moyen terme. Il est essentiel de concevoir une stratégie de coordination qui mette l'accent sur l'instauration et le maintien d'un climat de confiance entre le gouvernement, la société civile et la communauté internationale, et d'encourager une gestion plus transparente de l'information et du dialogue d'action.

- La notion de partenariats plus étroits : En Haïti, les partenariats ne pourront se bâtir qu'à partir d'alliances progressives avec des groupes ou des institutions pouvant incarner le changement et se regrouper autour d'un objectif commun de développement. Ces alliances constituent un risque dans le court terme, car elles connaîtront des reculs et même des ruptures. Il sera alors nécessaire de prévoir des scénarios de repli assurant le maintien de l'engagement.

- L'approche axée sur les résultats : Les résultats des programmes seront définis en fonction du contexte particulier d'Haïti. Les résultats devront être modestes, modifiables dans leur envergure et vérifiables dans la durée. Ils devront être définis non pas uniquement en terme de changements positifs réels mais en terme de préservation des acquis, c'est-à-dire de la prévention d'un changement négatif. Le recensement et le contrôle des facteurs de risque feront partie intégrante du suivi.

- La recherche d'une plus grande cohérence : En Haïti, il existe déjà une très grande cohérence entre la politique étrangère et le programme de coopération. Cette cohérence devra s'étendre et inclure tous les ministères et agences canadiens impliqués en Haïti.

Source : ACDI (2003), Haïti : Cadre de programmation pays, ACDI. 


\section{Notes}

1. Banque mondiale/Fonds monétaire international (2002), A Review of the Poverty Reduction Strategy Paper Approach, Banque mondiale, Washington DC.

2. En moyenne, entre 1999 et 2001, 845 nouvelles activités de développement ont été lancées chaque année au Mozambique.

3. Banque mondiale (2002), L'action du Groupe de la Banque mondiale dans les pays à faible revenu en difficulté, Rapport du groupe de travail, Banque mondiale, Washington DC. 


\section{Chapitre 9}

\section{Aide humanitaire, dispositifs de cofinancement des ONG et autres formes d'aide bilatérale}

Les organismes d'aide au développement dispensent l'aide étrangère non seulement dans le cadre de programmes par pays adaptés au contexte spécifique de chaque partenaire, mais aussi par le biais d'autres dispositifs dont la gestion suppose une forte implication du personnel au siège et qui font parfois l'objet d'un budget dédié séparé. Parmi ces dispositifs, figurent les programmes régionaux, l'aide humanitaire, les programmes visant des domaines fonctionnels tels que le développement du secteur privé ou la promotion de la bonne gouvernance, ou les dispositifs de cofinancement des ONG. Ils viennent parfois en complément des activités en cours dans les principaux pays partenaires ou sont mis en cuvre dans les pays en développement où il n'existe aucune autre activité de programme. Dans ce dernier cas, ces mécanismes peuvent élargir la portée géographique d'un programme d'aide étrangère mais risquent, ce faisant, de disperser ou de diluer les retombées des initiatives d'un donneur particulier en matière de coopération pour le développement. Lors de la conception et de la mise en œuvre de tels programmes, il convient de se pencher sur d'autres questions d'importance majeure, telles que la cohérence des programmes avec les priorités globales en matière de développement, leur compatibilité avec le modèle de partenariat, leurs effets sur le renforcement de la prise en charge locale, et d'assurer l'efficacité du processus de suivi et d'évaluation. Les programmes humanitaires posent des défis particuliers : ils impliquent notamment d'élaborer une approche stratégique adaptée à cette forme d'aide, de veiller à ce que les considérations humanitaires demeurent prédominantes par rapport aux objectifs de politique étrangère ou aux intérêts politiques, et de trouver l'équilibre entre l'aide humanitaire et l'utilisation des ressources au service du développement à long terme. 


\section{Programmes régionaux}

À l'échelon régional, les programmes d'aide étrangère peuvent être efficaces pour résoudre divers problèmes liés au développement qui ne sont pas limités par les frontières nationales. Le développement de l'Afrique australe, par exemple, est fortement tributaire de la situation économique de l'Afrique du Sud, de la modernisation des infrastructures régionales de transport et des politiques d'immigration. Dans la région pacifique, la surexploitation des ressources naturelles renouvelables, en particulier des ressources halieutiques, a des conséquences sur les moyens de subsistance de nombreuses petites communautés insulaires. De manière plus générale, la multiplication des déplacements et des migrations, ainsi que l'essor du commerce international des denrées alimentaires et des animaux ont rendu certaines régions plus vulnérables à la propagation des maladies transmissibles par delà les frontières.

Cette dimension régionale des questions de développement doit être prise en compte avant tout par les gouvernements nationaux à qui il incombe d'élaborer, en étroite collaboration, des politiques et des solutions régionales conjointes. Les organismes d'aide au développement peuvent apporter leur appui en favorisant une action collective et en renforçant la capacité des gouvernements partenaires de s'attaquer aux problèmes régionaux.

Afin de tenter de relever les défis liés au développement régional, les organismes d'aide au développement peuvent également choisir de soutenir et de financer le renforcement des capacités des organismes et des groupements régionaux. Les pays membres du CAD soutiennent ensemble une certain nombre de groupements régionaux, y compris la Communauté du développement de l'Afrique australe (SADC), la Coopération économique Asie-Pacifique (APEC) (voir ci-dessous) et l'Association sud-asiatique de coopération régionale (ASARC)*

\section{Un exemple : la Nouvelle-Zélande}

La Nouvelle-Zélande est un membre dynamique de plusieurs organisations et groupements de la région Asie-Pacifique. Elle participe à un ensemble d'initiatives destinées à aider les pays en développement de la région à s'intégrer à l'économie mondiale. À titre d'exemple, la Nouvelle-Zélande joue un rôle moteur au sein de l'APEC, dans les efforts déployés par l'Association pour analyser les causes de la crise financière asiatique. Elle soutient les initiatives en faveur du renforcement de la gouvernance financière et du gouvernement d'entreprise, et fournit un appui aux activités de renforcement des capacités, par l'intermédiaire du Programme de coopération économique et technique de l'APEC. La Nouvelle-Zélande a par

\footnotetext{
* Les pays membres du CAD soutiennent également les banques régionales de développement et les organisations multilatérales régionales, telles que le Forum du Pacifique Sud. Leurs contributions sont assimilées à une aide multilatérale.
} 
ailleurs financé la participation de dirigeants de pays de la région du Mékong à des formations en politique commerciale, dispensées à l'Institut du Mékong.

Enfin, les membres du CAD peuvent également concevoir et mettre en œuvre des programmes d'aide étrangère à un échelon régional, ce qui est particulièrement fréquent dans les domaines de l'agriculture, de la résolution des conflits, de l'environnement, de la santé et de la lutte contre le VIH/sida. La nécessité d'envisager les problèmes dans leur dimension régionale est, dans certains cas tels que ceux du VIH/sida ou des conflits, devenue une priorité afin d'améliorer l'efficacité et la durabilité des programmes bilatéraux dans les pays partenaires de la région. À titre d'exemple, le Canada finance un certain nombre de programmes régionaux ou panafricains majoritairement axés sur la santé et l'agriculture (voir ci-dessous).

\section{Un exemple : le Canada}

Le Canada finance un projet d'une durée de sept ans et d'un montant de 35 millions de dollars visant à diminuer la transmission du VIH/sida en Afrique de l'Ouest. Il soutient les efforts des personnes engagées dans le développement communautaire ou le domaine sanitaire, qui travaillent auprès des groupes à hauts risques situés le long des principaux axe de migration dans neuf pays d'Afrique de l'Ouest : le Bénin, le Burkina Faso, la Côte d'Ivoire, le Ghana, la Guinée, le Mali, le Niger, le Sénégal et le Togo. Ce projet porte sur la gestion des maladies sexuellement transmissibles, la sensibilisation des populations et en particulier les groupes vulnérables, y compris les vendeurs de médicaments illégaux. Il vise à favoriser l'éducation sanitaire, à encourager l'utilisation du préservatif, à inciter les personnes concernées à se faire soigner et il traite aussi du problème de la disponibilité des médicaments. Il est également axé sur la réhabilitation des groupes à hauts risques et est parvenu à faire comprendre aux populations que le VIH/sida est « l'affaire de tous ».

\section{Programmes centralisés}

Une grande partie des activités gérées par les organismes d'aide au développement se présentent sous la forme de programmes à vocation unique ou "fonctionnels ", les plus courants d'entre eux ayant pour objectif la promotion de la démocratie et d'une gouvernance saine, la défense de l'environnement, l'aide alimentaire pour le développement, le développement du secteur privé, la mise à disposition de bourses d'enseignement tertiaire et les programmes de bénévolat. Pour de nombreux pays membres du CAD, le programme à vocation unique le plus important a trait à l'aide d'urgence et l'aide humanitaire - qui seront abordées dans la prochaine section. La caractéristique principale des programmes centralisés est d'être conçus, gérés et financés à partir du siège de ces organismes. Même s'ils ne portent théoriquement que sur un seul problème de développement, ils peuvent comporter des activités englobant des questions plus vastes, telles que la participation, l'appropriation et l'égalité homme-femme, dans les cas où le domaine traité s'avère complexe, ce qui est le cas par exemple de l'amélioration de la sécurité. Les programmes centralisés offrent aux organismes d'aide au développement une flexibilité supplémentaire en matière de financement et une nouvelle façon de gérer les programmes. Leur gestion centralisée peut en faire des instruments plébiscités par des donneurs de petite et moyenne tailles qui souhaitent étendre leurs activités à des pays partenaires dans lesquels ils n'ont pas de personnel de développement en poste. 
Dans certains cas, c'est la nature même du programme qui impose la nécessité d'une conception et d'une gestion centralisées. Certains programmes de développement du secteur privé, par exemple, mettent en relation des entreprises et des associations professionnelles du pays du donneur avec des entités équivalentes dans les pays partenaires, et fournissent des financements, des prêts ou des garanties pour la création de joint ventures. Une gestion centralisée facilite ces activités. Parallèlement, les pays membres du CAD ont récemment modifié leur stratégie en matière de sécurité et la considèrent davantage comme un axe essentiel de la lutte contre la pauvreté et de la réalisation des Objectifs du millénaire pour le développement. Cette évolution pourrait entraîner une multiplication des programmes à vocation unique portant sur des aspects spécifiques de la réforme du secteur de la sécurité et de la bonne gouvernance. Dans cette optique, les Pays-Bas ont récemment mis au point un dispositif de financement destiné à améliorer l'efficacité d'une approche plus intégrée en faveur de la paix, de la sécurité et du développement (voir ci-dessous).

\section{Un exemple : les Pays-Bas}

Les Pays-Bas ont créé il y a peu un Fonds de stabilité pour financer des activités qui se situent à l'interface entre le maintien et de la consolidation de la paix, et pour lesquelles l'utilisation des outils d'évaluation classiques qui servent à déterminer si une activité relève ou non de l'APD risque de compromettre l'application d'une approche globale et intégrée des problèmes de sécurité ayant une importance primordiale pour la lutte contre la pauvreté et le développement durable. Ce Fonds a pour objectif de fournir une aide rapide et flexible aux activités nécessaires à la promotion de la paix, de la sécurité et du développement dans les pays et les régions où des conflits violents sont susceptibles d'éclater ou sont déjà en cours. Il est destiné à favoriser la politique étrangère intégrée mise en place par les Pays-Bas dans le domaine de la paix, de la résolution des conflits et du développement, et qui repose sur une démarche multidimensionnelle englobant l'analyse de la stabilité, la prévention des conflits, les opérations de médiation, de maintien et de rétablissement de la paix, notamment la réforme du secteur de la sécurité, et les programmes de démobilisation et de réintégration. Une évaluation est effectuée à la fin de chaque exercice budgétaire pour déterminer quelles activités peuvent bénéficier de l'APD.

Les bourses d'enseignement tertiaire représentent une catégorie particulière de programmes dont la gestion est souvent centralisée. Elles permettent aux élèves des pays en développement et en transition de poursuivre des études de niveau supérieur, la plupart du temps dans le pays qui accorde la bourse. En théorie, les élèves devraient, de façon à maximiser les répercussions de cet enseignement sur le développement, suivre des formations présentant un intérêt pour le développement et revenir dans leur pays d'origine à l'issue de la formation afin de mettre en pratique les connaissances et les compétences acquises. Les programmes de bourses ont toutefois été souvent utilisés en tant qu'outil de politique étrangère afin de promouvoir de bonnes relations entre les pays, l'apprentissage des langues et la connaissance de la culture. Cette tendance risque d'affaiblir l'intérêt de ces programmes à des fins de développement. Certains organismes d'aide au développement soucieux d'optimiser les retombées des programmes de bourses tertiaires sur le développement privilégient désormais les bourses d'études dans le pays partenaire ou dans les pays de sa région, ou bien encore les formations plus courtes 
directement liées au développement. Cette forme ancienne et répandue d'aide étrangère n'est pas souvent l'objet d'évaluations ou de contrôles officiels dans les pays membres du CAD. Ceux d'entre eux qui souhaitent améliorer l'impact sur le développement de leur programme de bourses pour l'enseignement tertiaire peuvent toutefois tirer certains enseignements d'une évaluation que la République tchèque a récemment consacrée à son programme de bourse (voir ci-dessous).

\section{Un exemple : la République tchèque}

Comme c'est le cas dans de nombreux pays membres du CAD, les bourses d'enseignement tertiaire constituent depuis longtemps un volet important $d u$ programme d'aide étrangère de la République tchèque. Les ambassades à l'étranger demandent au ministère de l'Éducation du pays partenaire de nommer des candidats, qui sont pour la plupart acceptés. De manière générale, les élèves étudient en République tchèque pendant six ans, une année étant consacrée à la formation linguistique, et sont originaires d'environ 70 pays. L'évaluation a révélé que bien qu'un pourcentage de places soit réservé aux pays les moins développés, ce quota n'est généralement pas atteint. Ce sont les étudiants qui choisissent leurs disciplines d'étude, lesquelles, pour la plupart, n'ont guère de rapport avec le développement. Seuls environ deux tiers des élèves terminent leurs études et parmi eux, entre 20 et $50 \%$ ne retournent alors pas dans leur pays d'origine. Les 13 à 33 \% d'étudiants qui ont mené à bien leurs études et regagné leur pays d'origine étaient quant à eux susceptibles de trouver un emploi intéressant.

Les programmes de bénévolat sont une autre forme ancienne et répandue d'aide étrangère. Quelques pays membres du CAD, dont l'Allemagne, les États-Unis et la Norvège, se sont dotés d'organismes spécialisés dans la mise en œuvre de programmes de bénévolat, alors que d'autres pays, comme la Nouvelle-Zélande et le Royaume-Uni, financent des ONG qui envoient des travailleurs bénévoles dans les pays en développement. Conformément aux nouveaux principes en matière de coopération pour le développement, qui privilégient le renforcement des capacités dans les pays partenaires plutôt que l'envoi d'experts sur le terrain, un certain nombre de changements sont à signaler dans les pratiques des pays membres du CAD relatives aux programmes de bénévolat. Ainsi la Finlande a progressivement interrompu son programme de bénévolat, à la suite d'une évaluation qui a conclu que son coût était trop élevé et son efficacité difficilement mesurable. En 1998, l'Australie a mis en place un nouveau programme, intitulé "Youth Ambassadors for Development ", qui consiste à envoyer de jeunes Australiens qualifiés en mission de courte durée, de 3 à 12 mois, dans des pays en développement de la région Asie-Pacifique. L'Irlande, qui disposait auparavant d'un vaste programme de bénévolat géré par une agence spécialisée, a lancé en 2004 l'initiative "Volunteer 21 " afin de recenser et de promouvoir des approches nouvelles en matière de bénévolat, telles que le bénévolat de courte durée et le recours aux technologies de l'information pour soutenir un bénévolat virtuel, et notamment le "parrainage électronique ".

Pour être conformes au modèle de partenariat, les programmes centralisés doivent être élaborés en consultation avec les gouvernements partenaires et être cohérents avec les objectifs nationaux en matière de lutte contre la pauvreté. Si un pays soutient des 
activités dans un pays partenaire où un programme bilatéral est déjà mis en œuvre, il devra veiller à ce que ces activités soient également cohérentes avec la stratégie bilatérale du donneur, et conçues en consultation avec le personnel de développement et les autres personnels concernés en poste dans le pays partenaire. En outre, ces activités ne doivent pas faire double emploi et ne pas compliquer les obligations du donneur relatives à la coordination. Dans tous les cas, mais en particulier dans les pays où il n'existe aucun programme bilatéral, il est essentiel de mettre en place des systèmes appropriés de suivi et d'évaluation d'impact.

\section{L'aide humanitaire}

Pour de nombreux pays membres du CAD, l'aide humanitaire représente le premier programme à vocation unique, même si l'ampleur et les méthodes de gestion de cette aide varient sensiblement d'un pays à l'autre. Dans certains cas, c'est le ministère des Affaires étrangères qui gère l'aide humanitaire, par l'intermédiaire d'un département ou d'un bureau dédié. Dans d'autres, l'attention croissante que portent les pouvoirs publics aux questions de paix et de sécurité explique en partie la création de départements plus importants qui sont chargés de suivre diverses questions de dimension mondiale et qui assument, dans le cadre de leurs attributions, la responsabilité de l'aide humanitaire et des secours d'urgence. Dans certains pays disposant d'un organisme dédié à l'aide au développement, le département chargé de l'aide d'urgence ou de l'aide humanitaire est parfois implanté au sein de cette structure, mais il existe une collaboration et une concertation étroites avec le ministère des Affaires étrangères. Dans certains cas, et surtout si le programme humanitaire représente un aspect important de l'aide étrangère, ces départements sont dotés de spécialistes et sont en mesure de réagir rapidement face aux événements se produisant dans le monde et aux demandes d'aide qui s'ensuivent, parfois par une mobilisation extrêmement rapide d'équipes d'urgence (voir ci-dessous).

\section{Un exemple : la Norvège}

La Norvège est un acteur de premier plan de l'aide humanitaire. La part de l'aide d'urgence et de l'aide humanitaire dans le programme national est de loin supérieure à la moyenne des pays du CAD. Ses bénéficiaires, parmi lesquels figurent particulièrement des pays en conflit, sont nombreux. Les activités sont gérées par un département dédié au sein du ministère des Affaires étrangères. La Norvège est dotée d'un système d'intervention d'urgence bien rodé - NOREPS qui a mis en place des ressources de secours, fournit une aide d'urgence et est relié à une base de ressources humaines d'urgence - NORSTAFF - composée de 300 professionnels expérimentés, répartis en 25 catégories d'emplois et mobilisables à très court terme.

La plupart des pays membres du CAD acheminent une proportion non négligeable de leur aide humanitaire par le biais d'ONG nationales et internationales et des principaux organismes internationaux: le Haut Commissariat des Nations unies pour les réfugiés (UNHCR), le Comité international de la Croix-Rouge (CICR), le Programme alimentaire mondial (PAM) et, pour les États membres de l'Union européenne, l'Office humanitaire de la Communauté européenne (ECHO). En ce qui concerne les organismes multilatéraux, les pays membres du CAD participent au budget de base et fournissent des contributions 
multibilatérales en réponse à des crises humanitaires spécifiques. Les contributions multibilatérales versées aux organismes d'aide au développement sont largement supérieures à celles destinées à financer le budget de base et reflètent le nombre et l'ampleur des urgences humanitaires.

L'augmentation des niveaux de l'aide humanitaire a contribué à la multiplication $d u$ nombre de ses canaux d'acheminement et nécessite un meilleur ciblage sur la coordination et l'impact de cette aide. Initiative intéressante à cet égard, la Procédure d'appel global interinstitutions des Nations unies (CAP), qui a été lancée en 1991, fournit un cadre d'action à l'intérieur duquel les intervenants peuvent analyser les différents contextes, évaluer les besoins et les moyens à mettre en œuvre, s'entendre sur les priorités, définir les objectifs et élaborer un Plan d'action humanitaire commun (CHAP) pour les atteindre. La CAP a permis de renforcer la coopération entre les gouvernements, les donneurs, les organismes d'aide au développement et les bénéficiaires et, plus encore, de lever des financements, notamment pour les « crises oubliées ".

L'intensification de l'action humanitaire, la complexité croissante des crises humanitaires et l'accroissement du nombre et des types d'organismes fournisseurs d'aide suscitent par ailleurs des inquiétudes quant aux répercussions négatives que pourrait entraîner une aide mal gérée ou distribuée sans aucun contrôle. Par conséquent, les donneurs se préoccupent de plus en plus des questions de qualité et de responsabilité et se recentrent sur les résultats à atteindre. Plusieurs pays membres du CAD ont ainsi élaboré des stratégies spécifiques d'orientation des programmes humanitaires, dans lesquelles sont énoncés les critères et les principes devant guider les opérations de soutien et les interventions, et les dispositifs à mettre en œuvre. L'engagement d'un certain nombre de pays membres du CAD auprès d'organismes extérieurs actifs dans le domaine de l'humanitaire est de nature de plus en plus stratégique. Ainsi, certains de ces membres concluent des accords de partenariat stratégique avec des organismes internationaux de premier plan et recherchent de nouveaux moyens de garantir la pertinence et l'efficacité de leur réponse aux crises. De plus en plus, les interventions déclenchées en réponse à des urgences particulières sont l'objet d'évaluations destinées à tirer des enseignements et à améliorer l'efficacité.

Le resserrement des liens entre les différents ministères pourrait également faciliter la mise en œuvre d'une approche plus stratégique par les pays membres du CAD. Dans cette optique, un examen du financement humanitaire consenti par chaque pays peut s'avérer utile. En effet, si certains pays financent des activités dans une multitude de pays partenaires, d'autres s'efforcent d'être plus ciblés sur des régions, de façon à être cohérents avec l'orientation stratégique de leur programme global. À titre d'illustration, l'Italie est fortement orientée sur les Balkans, alors que le premier programme humanitaire de l'Australie porte sur le Timor oriental.

Pour les pays membres du CAD, l'un des problèmes majeurs est de trouver l'équilibre entre l'utilisation de l'aide étrangère à des fins humanitaire et la coopération pour le développement à long terme. Face à l'accroissement de la demande d'aide d'urgence ou d'aide humanitaire, de nombreux pays s'efforcent de gérer la transition entre une aide d'urgence/humanitaire et une coopération pour le développement à long terme, et adoptent une perspective à échéance de plus en plus lointaine. Les stratégies d'aide à court terme ne sont plus considérées comme une finalité, mais comme faisant partie intégrante d'un processus de développement du système et de renforcement des capacités. De fait, certains pays membres du CAD affectent des fonds humanitaires au soutien du 
renforcement des capacités d'intervention en cas d'urgence dans les pays vulnérables aux catastrophes naturelles.

Dans certains cas, un pays partenaire qui était au départ bénéficiaire de l'aide humanitaire peut devenir petit à petit un pays partenaire de premier plan, comme cela a été le cas du Timor oriental (voir ci-dessous). Il convient toutefois de mettre en balance les besoins et les attentes des pays partenaires qui ont bénéficié d'une aide humanitaire à la reconstruction ou au redressement après conflit et les efforts des pays membres du CAD visant à axer leurs programmes bilatéraux sur un nombre restreint de principaux pays partenaires.

\section{Un exemple : l'Irlande}

L'aide apportée par l'Irlande au Timor oriental illustre bien comment une approche plus stratégique peut faciliter le passage d'une aide d'urgence et humanitaire à une coopération pour le développement à long terme. Initialement fondé sur l'aide d'urgence, l'engagement de l'Irlande s'est peu à peu orienté vers les interventions par projet que proposaient les ONG, puis vers une stratégie pluriannuelle issue d'une concertation entre gouvernements, dont le but essentiel est d'aider les habitants du Timor oriental à réaliser les objectifs énoncés dans leur plan de développement national. Le Timor oriental fait désormais partie des sept pays de programme de l'Irlande et la gestion du programme qui lui est consacré a été transférée de la section Urgence et redressement à la section Pays de programme.

Dans le cadre des efforts plus généraux visant à améliorer la gestion, la qualité, le contenu et la transparence des programmes humanitaires, une réunion des principaux acteurs a été organisée à Stockholm en juin 2003 et a rassemblé 19 membres du CAD. Elle a abouti à l'adoption d'un ensemble de Principes et bonnes pratiques pour l'aide humanitaire (voir encadré 9.1), d'un Plan de mise en œuvre de l'aide humanitaire et à la création d'un groupe de mise en œuvre. Les travaux de ce groupe se poursuivent et portent notamment sur une proposition de révision, à des fins de notification et de recensement statistique, de la définition actuelle de l'aide humanitaire publique telle qu'employée par le CAD/l'OCDE. La réunion de Stockholm a considéré que les principaux objectifs de l'aide humanitaire étaient de sauver des vies, d'atténuer les souffrances et de préserver la dignité humaine. Toutefois, dans certains pays membres du CAD, la politique étrangère et les objectifs politiques entre en ligne de compte dans l'attribution de l'aide humanitaire. Selon les participants à la réunion, cette aide ne doit pas être utilisée comme un instrument de politique étrangère, au contraire, il convient d'élaborer des instruments d'action plus adaptés en matière de politique étrangère pour analyser les facteurs à l'origine des conflits ou de l'instabilité. 


\section{Encadré 9.1. Principes généraux et bonnes pratiques pour l'aide humanitaire}

Un groupe composé des premiers fournisseurs d'aide humanitaire publique au niveau mondial et de quelques-uns des principaux organismes et experts dans ce domaine s'est réuni à Stockholm en juin 2003 et a adopté les principes généraux suivant (entre autres) qui doivent être au cœur d'une action humanitaire avisée :

- Respecter et promouvoir l'application du droit international humanitaire, du droit des réfugiés et des droits de l'Homme.

- Tout en reconnaissant la responsabilité première des États à l'égard des victimes de crises humanitaires à l'intérieur de leurs frontières, s'appliquer à mobiliser rapidement des financements souples, en vertu de l'obligation qui incombe à la collectivité de faire de son mieux pour répondre aux besoins humanitaires.

- Répartir les financements humanitaires à proportion des besoins, et sur la base d'une évaluation de ces besoins.

- Inviter les organismes chargés de l'exécution de l'aide humanitaire à assurer, dans toute la mesure du possible, une participation adéquate des bénéficiaires à la conception, à la mise en œuvre, au suivi et à l'évaluation de la réponse humanitaire.

- Renforcer la capacité des pays et communautés locales affectés de prévenir les crises humanitaires, de s'y préparer, d'en atténuer les effets et d'y faire face, afin de faire en sorte que les gouvernements et les administrations locales soient mieux à même d'assumer leurs responsabilités et de coordonner efficacement leur action avec celle des partenaires œuvrant dans le domaine humanitaire.

- Dispenser l'aide humanitaire selon des modalités qui favorisent le redressement et le développement à long terme, en s'appliquant à faciliter, le cas échéant, la préservation de moyens d'existence durables ou le retour à de tels moyens ainsi que le remplacement progressif des secours humanitaires par des activités de reconstruction et de développement.

- Reconnaître et œuvrer à faire reconnaître le rôle central et unique des Nations unies en tant que chef de file et coordinateur de l'action humanitaire internationale, le rôle spécifique du Comité international de la Croix-Rouge ainsi que le rôle primordial des Nations unies, du Mouvement international de la Croix-Rouge et du Croissant-Rouge et des organisations non gouvernementales dans la mise en œuvre de l'action humanitaire.

\section{Cofinancement des ONG et des autres organismes de la société civile}

Tous les pays membres du CAD allouent, dans le cadre de leur aide étrangère, des crédits à des organismes de la société civile destinés à soutenir leurs activités en matière de développement ou à mettre en œuvre des activités pour le compte d'organismes d'aide au développement. Si la plus grande partie de ces crédits est affectée à des ONG nationales actives dans le domaine du développement, certains pays membres du CAD financent d'autres types d'organismes de la société civile, dont des fondations politiques, des fondations caritatives, des associations religieuses, des groupes communautaires, des syndicats et des organismes de formation et de recherche. Parmi ces derniers, certaines fondations privées - en particulier des fondations caritatives basées aux États-Unis - sont devenues d'importants bailleurs de fonds pour l'aide au développement, principalement d'origine privée, et des acteurs de plus en plus primordiaux du développement. Dans les économies en développement ou en transition, les groupes de la société civile ne sont 
financés directement que par un nombre restreint de pays membres du CAD, mais ils peuvent toutefois nouer des partenariats avec des groupes situés dans les pays membres du CAD et par conséquent bénéficier d'un financement indirect.

On considère généralement que les ONG et les autres organismes de la société civile fonctionnent selon des modalités différentes de celles des organismes publics d'aide au développement. En nouant des partenariats avec des ONG locales et des organismes communautaires, ceux-ci peuvent intervenir plus facilement dans des régions inaccessibles ou auprès de communautés isolées. Ces organismes mènent généralement une action efficace auprès des groupes hautement vulnérables tels que les travailleurs vivant du commerce sexuel, qui se méfient parfois des stratégies mises en place par les autorités. Leurs programmes sont souvent mis en œuvre par des ressortissants du pays partenaire. Travailler par l'intermédiaire des ONG ou des organismes de la société civile peut également présenter un intérêt dans le cas des pays peu performants, des faillites d'État, de conflits ou de situations post-conflit. Dans les cas où les gouvernements sont contraints de mettre un terme à leur aide au développement, les ONG peuvent s'avérer extrêmement utiles pour acheminer l'aide humanitaire en cours. On estime par ailleurs que les ONG sont la vitrine des pays membres du CAD, aussi bien sur le territoire national qu'à l'étranger. Leurs activités sont parfois mieux connues du public que celles des organismes publics d'aide au développement, et certains organismes de la société civile sont fortement impliqués dans la formation en matière de développement.

La proportion d'APD acheminée par le biais d'ONG varie considérablement entre les différents pays du CAD, de même que l'existence de principes officiels destinés à encadrer les relations avec les donneurs. Certains pays membres du CAD financent de nombreuses ONG selon des critères relativement souples (voire sans aucun critère) alors que d'autres limitent leur financement aux ONG les plus grandes ou les plus reconnues, ou en fonction de critères soigneusement élaborés.

Les pays membres du CAD ont recours à une palette de dispositifs de financement pour les ONG, et combinent fréquemment leur utilisation. Les pays qui ne peuvent prévoir leurs engagements budgétaires que d'une année sur l'autre obligent les ONG à fonctionner au sein d'un environnement aléatoire et à travailler sur des projets de développement qui, par nature, sont forcément mieux adaptés à des activités de plus long terme. Conscients des difficultés que cette situation implique pour les ONG, et dans le cadre d'une approche de plus en plus stratégique, certains pays ont évolué vers un financement pluriannuel pour quelques ONG - généralement les plus grandes et les plus anciennes. En plus de telles dispositions, les ONG peuvent bénéficier des fonds alloués par les donneurs à des problèmes spécifiques, par l'intermédiaire de programmes de financement spéciaux, tels que ceux consacrés à l'aide humanitaire, à l'hygiène de la reproduction ou à la gouvernance, même si certains pays membres du CAD s'efforcent de diminuer le nombre des programmes de financement afin d'en réduire les coûts de gestion et d'en accroître l'efficacité. Nombre de ces programmes fonctionnent sur la base d'un cofinancement, le financement apporté par l'ONG provenant également d'autres sources, souvent caritatives.

Dans la plupart des pays du CAD, les priorités géographiques du programme public d'aide au développement ne s'appliquent pas aux activités des ONG bénéficiaires d'un financement public. Cette disposition permet au programme public de dépasser les limites géographiques de ses activités et de financer des interventions dans d'autres pays, particulièrement à des fins humanitaires. Cependant, cette pratique accentue la 
dispersion, n'est pas conforme aux tentatives de ciblage et réduit les possibilités de synergies entre les activités des ONG et les programmes bilatéraux d'État à État. Certains pays ont pris des mesures visant à encourager les ONG partenaires à recentrer leurs activités dans des pays de programme. Parmi ces mesures figurent l'élaboration de critères officiels permettant de définir les activités des ONG et une hausse du financement des activités dans les principaux pays partenaires (voir ci-dessous).

\section{Un exemple : l'Autriche}

Les ONG autrichiennes jouent un rôle fondamental dans le programme national de coopération pour le développement : en effet, elles mettent en œuvre plus de $50 \%$ du programme d'aide bilatérale du ministère des Affaires étrangères. Les activités des ONG sont régies par des lignes directrices et divers critères. Les ONG perçoivent des subventions pouvant aller jusqu'à $50 \%$ du coût d'un projet lorsqu'elles exercent leurs activités dans les principaux pays partenaires, jusqu'à $25 \%$ dans les autres pays en développement et jusqu'à $75 \%$ pour les projets en Europe du Sud-Est, en Europe orientale et en Asie centrale. Les ONG ont dû revoir leurs priorités régionales et sectorielles pour tenir compte du fait que l'Agence autrichienne pour le développement se recentre de plus en plus sur ses propres priorités et sur les activités avec ses pays de coopération, ainsi que sur le lancement de programmes par pays et par secteur. Les ONG ont accueilli favorablement l'idée de cette réorientation vers un recentrage sur les activités de développement et une plus grande convergence de l'action sur le plan politique. De nouvelles relations de travail ont déjà été nouées dans un certain nombre de pays.

En plus des efforts à déployer pour améliorer la cohérence géographique des activités des ONG, une tâche délicate reste à accomplir, à savoir, veiller à assurer la conformité des activités des ONG avec les politiques et les priorités du gouvernement. Cette mission peut être facilitée par la mise en place de programmes de financement pluriannuel et par l'élaboration conjointe de politiques, mais elle s'avère plus difficile lorsqu'un grand nombre d'ONG bénéficie d'un financement public pour des projets de petite envergure. Mettre davantage l'accent sur le suivi et l'évaluation des projets peut avoir des retombées positives, mais cela soulève des problèmes de gestion considérables également pour les organismes d'aide au développement.

La proportion que représente le financement public dans le budget global d'un organisme peut parfois servir de critère pour déterminer dans quelle mesure les priorités des gouvernements peuvent et doivent orienter les activités des ONG. Dans certains pays membres du CAD, c'est l'État qui fournit aux organismes la quasi-totalité de leur financement, et on peut par conséquent déplorer un manque d'indépendance chez les ONG, alors que dans d'autres pays, le montant des fonds que perçoivent certains organismes d'aide au développement de la part de sources non gouvernementales peut être au moins égal à celui du financement public; il arrive même que certains organismes limitent volontairement la part de leur financement public de façon à conserver leur indépendance.

Certains pays membres du CAD ont pris des mesures actives pour inciter les ONG à adopter une vision sur le long terme plus stratégique, à définir plus clairement leurs priorités, à déterminer plus explicitement leurs domaines d'expertise et à se recentrer sur l'évaluation. Dans cette optique, des organismes d'aide au développement financent des initiatives portant sur le renforcement des capacités, de façon à permettre aux ONG de soutenir le développement des compétences et des politiques dans ces domaines. Ces 
mesures sont particulièrement importantes pour les ONG de taille plus restreinte qui risquent d'être évincées par des organisations plus grandes et plus structurées.

Le soutien apporté par les organismes de coordination peut être l'un des aspects de ce processus de renforcement des capacités. Les organismes de coordination des ONG peuvent faciliter le dialogue avec le gouvernement et l'élaboration de stratégies conjointes, créer un espace de partage des informations et sont peut-être mieux à même de représenter plus efficacement les intérêts et les opinions de leurs membres que si ces derniers devaient les exprimer individuellement. Les organismes de coordination peuvent s'apercevoir qu'ils ont davantage de poids dans le processus d'élaboration des politiques étant donné qu'ils facilitent la communication avec le gouvernement. Dans certains pays membres du CAD, les représentants des ONG ou des organismes de coordination des ONG siègent parfois aux comités consultatifs sur le développement et remplissent une mission officielle dans l'examen et la formulation des politiques. 


\section{Chapitre 10}

\section{Aide multilatérale et contributions aux fonds mondiaux}

Les contributions aux institutions multilatérales constituent un important moyen d'acheminement de l'APD et de l'aide publique des pays membres du CAD. Pour de nombreux pays, les organisations multilatérales offrent l'avantage de pouvoir mobiliser des volumes importants de ressources et coordonner les réponses des donneurs aux problèmes du développement dans le monde. Cependant, dans beaucoup de pays membres du CAD, la gestion de l'aide multilatérale et sa coordination avec l'aide bilatérale mériteraient davantage d'attention. Certains pays commencent à adopter vis-à-vis de l'aide multilatérale une démarche proactive, notamment en évaluant la performance des organisations multilatérales sur le terrain. Les fonds mondiaux, qui en principe sont consacrés à des domaines spécifiques comme la santé ou l'environnement, offrent aux pays membres du CAD un moyen différent et relativement nouveau d'aborder le problème du développement au niveau régional ou mondial. Ces fonds présentent un certain nombre d'avantages, mais suscitent encore des interrogations quant à la transparence de leur fonctionnement et la mesure dans laquelle ils pratiquent une politique de partenariat. 


\section{Aide multilatérale}

Pour le CAD, les contributions ne peuvent être comptabilisées dans l'aide multilatérale que si :

- Elles sont versées à une organisation intergouvernementale qui consacre une part importante, voire la totalité, de ses activités aux pays en développement (ou en transition).

- Ces contributions sont regroupées avec les autres montants versés à l'organisation de telle façon qu'elles perdent leur identité et deviennent partie intégrante des actifs financiers de ces organisations.

- Les contributions regroupées sont décaissées à l'initiative de l'organisation.

Toute APD ou aide publique qui ne remplit pas ces critères est classée dans l'aide bilatérale. Cela inclut l'aide multibilatérale, c'est-à-dire l'aide extérieure versée à titre volontaire par les donneurs à un organisme multilatéral, en supplément des contributions générales, et qui est affectée à des activités spécifiques.

L'aide multilatérale est acheminée par de nombreuses organisations. Les principales catégories sont les suivantes :

- Les banques multilatérales de développement : le groupe de la Banque mondiale, avec son Association internationale de développement (IDA) et les quatre banques régionales de développement, avec leurs guichets de prêts assortis de conditions libérales (la Banque africaine de développement [BAfBD], la Banque asiatique de développement, la Banque européenne pour la reconstruction et le développement [BERD] et la Banque interaméricaine de développement [BID]).

- Des institutions des Nations unies comme le Programme des Nations unies pour le développement (PNUD), Fonds des Nations unies pour l'enfance (UNICEF), Programme alimentaire mondiale (PAM) et Haut Commissariat des Nations unies pour les réfugiés (HCR).

- La Communauté européenne (pour les États membres de l'Union européenne). Il s'agit ici essentiellement du Fonds européen de développement (FED) et des activités de développement financées sur les ressources propres de la Commission. Dans les statistiques du CAD, elles sont fictivement réaffectées à chaque État membre sur une base proportionnelle.

L'aide multilatérale et les relations stratégiques avec les organisations multilatérales sont gérées de façon assez différente selon les pays membres du CAD : pour les banques multilatérales de développement, en particulier la Banque mondiale, dans de nombreux pays c'est le ministère des Finances qui gère les contributions générales et conduit le dialogue politique, tandis que le ministère des Affaires étrangères ou l'administration chargée du développement, lorsqu'elle existe, se charge des relations avec la plupart des autres organisations multilatérales qui s'occupent de développement. Compte tenu du rôle important qu'il joue pour le développement, ce ministère (ou cette administration) 
comporte en général un département spécifique, divisé en plusieurs sections chargées respectivement des relations avec les institutions des Nations unies, l'Union européenne (pour les États membres de cette Union) et les institutions financières internationales, même si le principal responsable reste le ministère des Finances.

S'agissant des institutions plus spécialisées des Nations unies, les relations peuvent relever de plusieurs ministères. La gestion des contributions générales et des relations avec les instances de direction de l'Organisation mondiale de la santé, par exemple, relève généralement du ministère de la Santé, mais celui-ci peut s'appuyer sur les spécialistes du développement et les experts techniques de l'administration du développement ou du ministère des Affaires étrangères.

La gestion du développement et de la cohérence générale des politiques au sein de l'Union européenne est un domaine complexe qui demande que les États membres se dotent d'une structure spécifique. Les États membres, le Parlement européen et la Commission elle-même jouent un rôle dans le processus de décision ; or, leurs priorités sont souvent différentes, parfois même contradictoires. La mise en œuvre des activités de coopération et de développement incombe essentiellement à la Commission. Les principaux acteurs en sont la direction générale (DG) Relations extérieures, la DG Développement, ECHO et Europe Aid, qui est l'Office créé en 2001 pour mettre en œuvre les instruments d'aide extérieure de la Commission (voir aussi encadré 10.1). L'exécution des programmes d'aide extérieure dans les pays partenaires est assurée par le biais des délégations locales de la Commission.

Les pays membres du CAD consacrent en moyenne à l'aide multilatérale près de $30 \%$ de leur APD brute. Jusqu'ici, peu d'études ont véritablement analysé les principaux facteurs qui déterminent le niveau des contributions des pays membres du CAD aux organisations multilatérales. Toutefois, une étude consacrée au financement des organisations multilatérales entre 1970 et 2000 donne un certain nombre d'indications. L'essentiel de la charge financière est assumé par les petits donneurs. Le financement des organismes multilatéraux est un poste très résiduel dans les budgets globaux de l'aide. Or, si l'on excepte la Commission européenne, les organisations multilatérales ne sont qu'une voie d'acheminement de l'aide aux pays en développement. Dans les États membres de l'Union européenne en revanche, les contributions à la Commission européenne ne remplacent pas les contributions aux autres organisations multilatérales. Enfin, ni la conjoncture économique dans laquelle se trouve un pays ni son taux de croissance ne modifient sa part de responsabilité financière ${ }^{1}$.

Deux tendances récentes se dégagent depuis peu dans la politique d'aide multilatérale des pays membres du CAD. Premièrement, certains pays recentrent leurs financements sur un plus petit nombre d'organisations multilatérales, en fonction de leurs priorités et de leurs approches (voir ci-dessous).

Une deuxième tendance est l'établissement par certains membres du CAD de documents d'orientation stratégique ou de cadres d'évaluation qui permettent de mettre en évidence les points forts et faibles des grandes institutions multilatérales, d'évaluer l'impact des engagements existants, l'efficacité de l'institution et son adéquation aux priorités des gouvernements (voir ci-dessous). Certains mettent aussi l'accent sur les performances locales des organisations multilatérales, en faisant suivre et évaluer leur action dans les pays partenaires par le personnel des pays membres du CAD présent sur le terrain (voir ci-dessous). Il faudrait que tout ceci s'inscrive dans le cadre d'un effort plus 


\section{Encadré 10.1. Programmes d'aide extérieure gérés par l'Union européenne}

L'Union européenne est un donneur atypique en ce sens qu'elle joue un double rôle dans le développement, en tant qu'important fournisseur d'aide directe aux pays partenaires, et en même temps coordonnateur des programmes d'aide extérieure des États membres de l'Union. La Commission européenne est le plus gros apporteur d'aide multilatérale au monde, de même que l'Union européenne est le plus grand marché unique et le principal partenaire commercial de la plupart des pays en développement.

La Commission européenne est un organe exécutif responsable devant le Parlement européen et les États membres réunis en Conseil. Le Conseil des affaires générales et relations extérieures, qui est l'une des neuf configurations du Conseil, est responsable des relations extérieures de l'Union européenne, y compris dans le domaine économique et dans ceux de la coopération au développement et de l'aide humanitaire. Le Conseil est chargé de coordonner l'action des États membres dans ces domaines et d'assurer la cohérence entre les différents aspects des relations extérieures de l'Union. Dans le système actuel, il est présidé par le ministre des Affaires étrangères de l'État membre qui assure pour six mois la présidence tournante de l'Union européenne, aidé du Secrétaire général du Conseil.

La Commission européenne et le Conseil de l'Union européenne ont adopté en novembre 2000 une importante déclaration commune sur la politique de développement. Cette déclaration, qui mettait la réduction de la pauvreté au centre de la politique de développement de l'Union européenne, répartissait l'action en six grands domaines : i) le commerce et le développement ; ii) l'intégration et la coopération régionales ; iii) l'appui aux politiques macroéconomiques et la promotion d'un accès équitable aux services sociaux ; iv) les transports ; v) la sécurité alimentaire et le développement rural durable ; enfin vi) le renforcement des capacités institutionnelles, tout ceci devant se faire avec le souci des droits de l'homme, de l'environnement, de l'égalité entre les sexes et de la bonne gouvernance. La priorité serait donnée aux pays les moins avancés et aux autres pays à faible revenu.

La Commission européenne finance les activités de développement d'une part, via les lignes du budget de l'Union européenne affectées à l'action extérieure et d'autre part, via le FED. Les fonds pour l'action extérieure qui couvrent plus d'activités que ce qui relève à proprement parler de l'APD, sont alimentés par les contributions des États membres au budget ordinaire et par des ressources propres de la Commission comme les droits de douane. Le Fonds européen de développement est un programme pluriannuel d'appui aux pays en développement de la région Afrique, Caraibes et Pacifique (ACP), à l'Afrique du sud, aux territoires d'outre-mer des pays membres, et à certains fonds thématiques tels que l'aide alimentaire. Il est financé par des contributions volontaires des États membres et gérés par la DG Développement, Europe Aid et, pour l'aide d'urgence, ECHO. Les fonds pour l'action extérieure et le FED se répartissent entre un certain nombre d'instruments de programmation, notamment d'importants programmes régionaux/géographiques.

large de renforcement des liens et de l'apprentissage mutuel entre les acteurs de l'aide multilatérale et bilatérale. Les documents d'orientation stratégique pourraient servir de base aux décisions sur la suite des relations et sur les niveaux de financement et définir la stratégie globale du gouvernement pour la gestion future du partenariat. 


\section{Un exemple : l'Irlande}

L'Irlande a adopté vis-à-vis des institutions des Nations unies auxquelles elle contribue une approche plus sélective et plus ciblée; elle a augmenté ses contributions à celles qui correspondent à ses propres objectifs, en particulier la réduction de la pauvreté ; parallèlement, elle a ramené de 35 à 20 le nombre des institutions auxquelles elle contribue, en se retirant de celles où ses contributions étaient symboliques ou qui cadraient mal avec les objectifs d'ensemble de sa politique. Elle s'est fixé pour le choix de ses partenaires multilatéraux un ensemble de critères : ils doivent avoir pour objectif déclaré la réduction de la pauvreté ; être propres à contribuer à la réalisation des objectifs du millénaire pour le développement; témoigner d'une gestion solide et d'une volonté de réforme et de coordination avec les partenaires multilatéraux et bilatéraux, notamment dans le cadre de dispositifs de financement collectif dans les pays partenaires; et appliquer un système de compte rendu qui garantisse la transparence. C'est sur cette base que l'Irlande s'est engagée de façon plus importante et plus stratégique auprès de cinq institutions : le PNUD, le HCR, l'UNICEF, le PAM et le Fonds des Nations unies pour la population (FNUAP). Elle s'est par ailleurs dotée d'une capacité accrue de suivi en siégeant aux Conseils de ces organismes et en organisant des consultations bilatérales annuelles.

\section{Un exemple : l'Australie}

Le Fonds international de développement agricole (FIDA) a été créé en 1977 pour accorder des prêts et des subventions visant à atténuer la pauvreté rurale. L'Australie, qui en a été membre fondateur, y a consacré au total 50 millions USD. Cependant, pour diverses raisons, elle a aujourd'hui décidé de s'en retirer : i) le FIDA n'a que peu d'utilité pour les pays d'Asie du Sud-Est et du Pacifique qui sont prioritaires pour l'aide de l'Australie, car il est essentiellement centré sur l'Afrique; ii) il ne présente pas d'avantage comparatif par rapport à d'autres organisations qui s'impliquent davantage dans le développement rural de la région; et iii) il n'a pas répondu aux objections formulées par l'Australie concernant sa direction. Le FIDA étant une organisation basée sur un traité, l'Australie est obligée d'entamer une procédure formelle de retrait de ce traité, qui implique la présentation au Parlement des raisons de ce retrait et l'organisation des auditions publiques.

\section{Un exemple : Canada, Danemark, Allemagne, Pays-Bas, Norvège, Suède, Suisse et Royaume-Uni}

Huit pays donneurs ont mis en place en 2002, pour réaliser des évaluations périodiques des performances des organisations multilatérales au niveau local, un réseau dénommé Multilateral Organisations Performance Assessment Network (MOPAN). Le but de ce réseau est d'améliorer la remontée des informations relatives aux performances multilatérales du terrain vers l'administration centrale, afin de permettre aux pays membres d'être plus efficaces en tant que partenaires, de mieux rendre compte à leurs Parlements nationaux et de mieux connaître les travaux et les priorités des organisations en question. En 2003, un exercice pilote centré sur le secteur de la santé a évalué les performances de l'OMS, de l'UNICEF, de la Banque mondiale et des Banques africaine, asiatique et interaméricaine de développement 
dans huit pays partenaires: Bangladesh, Ghana, Inde, Malawi, Mozambique, Nicaragua, Ouganda et Viêt-nam. Cet exercice a montré que les organisations multilatérales sont perçues comme ayant assez efficacement contribué à axer davantage les politiques nationales de santé sur la pauvreté, mais comme n'ayant pas contribué sensiblement à renforcer les capacités locales²

Certaines préoccupations se sont récemment exprimées quant à la "bilatéralisation " croissante de l'aide multilatérale, c'est-à-dire l'importance de plus en plus grande de l'aide extérieure acheminée par les donneurs via l'organisation multilatérale au titre de contributions volontaires à affectation spéciale. Cette aide multibilatérale est devenue une composante majeure du financement de certaines institutions spécialisées des Nations unies. Ainsi, sur le budget global de l'OMS pour 2002-2003, $38 \%$ seulement correspondaient aux contributions ordinaires des États membres, les $62 \%$ restants étant constitués de contributions volontaires ${ }^{3}$. De même, les contributions volontaires des États membres du CAD sont la principale source des fonds du HCR; en 2002, $20 \%$ seulement des contributions volontaires n'avaient pas d'affectation spéciale et $43 \%$ en avaient une ${ }^{4}$. Les avis divergent au sein du CAD concernant cette aide multibilatérale. Si elle représente des volumes importants, et surtout si elle est affectée à des secteurs ou des pays particuliers, elle risque de modifier les priorités de l'Organisation en cause et d'en affaiblir le caractère multilatéral. D'un autre côté, certains membres du CAD considèrent que les contributions volontaires sont un moyen essentiel d'exercer une influence, d'orienter les programmes et d'accroître l'efficacité des Organisations.

\section{Fonds mondiaux}

Les fonds mondiaux - comme le Fonds pour l'environnement mondial (FEM), l'Alliance mondiale pour les vaccins et la vaccination (GAVI) et le Fonds mondial de lutte contre le sida, la tuberculose et le paludisme (GFATM) - s'affirment comme des mécanismes importants de financement des activités de développement. Les fonds mondiaux se distinguent des organisations multilatérales en ce sens que, comme le dit une étude ${ }^{5}$, « ils constituent un instrument financier dont l'objet premier est d'attirer, de gérer et de distribuer des fonds ", et qu'ils présentent les caractéristiques suivantes :

- Partenariat public/privé : le secteur privé y est souvent contributeur financier ou partenaire de cofinancements, et les organes de direction peuvent comprendre des représentants du secteur privé, de la société civile et d'autres parties prenantes.

- Indépendance : ils ne dépendent d'aucune institution spécifique et sont constitués en entités distinctes dotées d'une personnalité juridique indépendante, en tant qu'alliance dotée, à des fins juridiques, d'un bras financier ou en tant que mécanisme financier pour des accords internationaux.

- Mission spécifique : la mission est souvent centrée sur un domaine spécifique.

La structure et le mode de gouvernance de trois fonds mondiaux - le FEM, le GAVI et le GFATM - sont décrits au tableau 10.1.

Les pays membres du CAD contribuent de façon importante aux fonds mondiaux et dans certains cas à leur gouvernance. Ces fonds ont pour eux un certain nombre d'avantages majeurs :

- Ils peuvent constituer des filières importantes pour le financement de biens publics mondiaux. 
- Ils peuvent obtenir des apports supplémentaires des sources publiques, lorsque celles-ci ne souhaitent pas développer les programmes bilatéraux ou fournir un soutien financier supplémentaire aux organisations internationales en place.

- Ils ont des chances d'être plus innovants et plus souples dans leur action.

- Ils permettent d'attirer la participation du secteur privé, de la société civile et d'autres parties prenantes.

- L'utilisation des fonds n'obéit aux directives d'aucun donneur ou organisme particulier et se concentre davantage sur les besoins des pays partenaires.

Cela dit, ces fonds présentent aussi des défauts ; les plus couramment cités sont les suivants :

- Le centrage de leur mission sur un domaine spécifique ne permet pas d'exploiter les synergies au niveau de l'élaboration des politiques, et il est en contradiction avec le principe du partenariat piloté par le pays en développement qui sous-tend les priorités et stratégies nationales, notamment la stratégie de réduction de la pauvreté.

- Ils peuvent faire double emploi avec des structures existantes et par conséquent accroître les coûts de transaction.

- Ils n'ont pas la responsabilité démocratique des organisations multilatérales et des gouvernements.

- Ils peuvent ne pas attirer des financements supplémentaires mais être utilisés comme filières de remplacement pour l'aide étrangère.

Ces avantages et ces inconvénients peuvent influer sensiblement sur les décisions de financement des pays membres du CAD, particulièrement à un moment où de nombreux pays souhaitent donner à leur engagement auprès des organisations multilatérales en général un caractère de plus en plus stratégique. Ainsi, par exemple, la représentation aux Conseils des fonds mondiaux varie beaucoup ; dans certains cas, les donneurs disposent automatiquement d'un siège au Conseil, tandis que dans d'autres cas un même siège peut être partagé ou occupé de façon tournante entre plusieurs donneurs. Si les pays membres $\mathrm{du} C A D$ veulent s'orienter vers un engagement stratégique auprès des fonds mondiaux, il faut qu'ils puissent s'engager auprès du Conseil et des autres donneurs sur des questions aussi importantes que la gouvernance, les priorités, les questions de durabilité et les politiques de partenariat. Ils peuvent aussi jouer un rôle important en veillant ensemble au niveau du Conseil comme sur le terrain à ce que les fonds mondiaux respectent les pratiques internationalement admises, notamment en ce qui concerne le partenariat pour le développement et l'appropriation et la prise en main par les pays bénéficiaires. Exemple d'action commune de suivi de l'impact d'un fonds mondial dans les pays bénéficiaires : la Global Fund (for AIDS, TB and Malaria) Tracking Study en cours dans quatre pays partenaires, financée par le Danemark, l'Irlande, les Pays-Bas et le Royaume-Uni.

On trouvera ci-dessous un tableau comparatif de la structure et du mode de gouvernance de trois grands fonds mondiaux. 
Tableau 10.1. Fonds mondiaux

\begin{tabular}{|c|c|c|c|}
\hline & FEM & GAVI & GFATM \\
\hline Mission & $\begin{array}{l}\text { Le FEM aide les pays en } \\
\text { développement à financer des projets } \\
\text { et programmes de protection de } \\
\text { l'environnement mondial. II s'agit } \\
\text { de projets relatifs à la biodiversité, au } \\
\text { changement climatique, aux eaux } \\
\text { internationales, à la dégradation } \\
\text { des sols, à la couche d'ozone et aux } \\
\text { polluants organiques rémanents. }\end{array}$ & $\begin{array}{l}\text { Le GAVI a été créé pour réunir les } \\
\text { compétences et l'expérience de } \\
\text { multiples partenaires en matière de } \\
\text { vaccination. II fournit aux pays des } \\
\text { moyens financiers pour acheter les } \\
\text { vaccins et fournitures connexes et } \\
\text { assurer la vaccination. }\end{array}$ & $\begin{array}{l}\text { Le GFATM a été créé pour accroître les } \\
\text { moyens de lutte contre trois des } \\
\text { pathologies les plus dévastatrices à } \\
\text { l'échelle mondiale - sida, tuberculose et } \\
\text { paludisme - et les affecter aux zones } \\
\text { qui en ont le plus besoin. }\end{array}$ \\
\hline Année de création & 1991, restructuré en 1994 & 1999 & 2002 \\
\hline $\begin{array}{l}\text { Principaux donneurs } \\
\text { bilatéraux }\end{array}$ & & $\begin{array}{l}\text { États-Unis, Norvège, Pays-Bas, } \\
\text { Royaume-Uni. }\end{array}$ & $\begin{array}{l}\text { États-Unis, France, Allemagne, Italie, } \\
\text { Japon. }\end{array}$ \\
\hline $\begin{array}{l}\text { Rôle des organisations } \\
\text { internationales }\end{array}$ & $\begin{array}{l}\text { La Banque mondiale, le PNUE et } \\
\text { le PNUD sont les agents d'exécution et } \\
\text { les principaux responsables de la mise } \\
\text { au point des programmes. }\end{array}$ & $\begin{array}{l}\text { Achat de vaccins, via l'UNICEF, } \\
\text { expertise technique et opérationnelle } \\
\text { fournie par l'OMS. }\end{array}$ & $\begin{array}{l}\text { Soutien et assistance technique pour } \\
\text { les propositions de programmes-pays } \\
\text { et leur exécution. }\end{array}$ \\
\hline Rôle du secteur privé & $\begin{array}{l}\text { Aucun rôle dans la gouvernance ou } \\
\text { en tant que contributeurs financiers } \\
\text { directs. Participation à certains } \\
\text { cofinancements et projets communs } \\
\text { au niveau des pays. }\end{array}$ & $\begin{array}{l}\text { Rôle clé en tant que partenaires, } \\
\text { notamment dans le développement } \\
\text { et la fourniture des vaccins. } \\
\text { La fondation Gates est le principal } \\
\text { donneur. }\end{array}$ & $\begin{array}{l}\text { Contributeurs financiers (environ } 5 \% \text { ) } \\
\text { avec représentation au Conseil . }\end{array}$ \\
\hline Rôle de la société civile & $\begin{array}{l}\text { Consultation et participation aux } \\
\text { réunions du Conseil, éventuellement } \\
\text { bénéficiaire de financements via } \\
\text { les agents d'exécution ; les petites } \\
\text { subventions vont directement aux } \\
\text { ONG locales. }\end{array}$ & Représentation limitée au Conseil . & $\begin{array}{l}\text { Membres du Conseil avec droit de vote } \\
\text { et bénéficiaires de financements. }\end{array}$ \\
\hline Dispositions fiduciaires & $\begin{array}{l}\text { La Banque mondiale est } \\
\text { administrateur fiduciaire. }\end{array}$ & $\begin{array}{l}\text { L'UNICEF est administrateur } \\
\text { fiduciaire. }\end{array}$ & $\begin{array}{l}\text { La Banque mondiale est administrateur } \\
\text { fiduciaire, sous-administrateur au } \\
\text { niveau national, les versements sont } \\
\text { faits directement à l'État concerné. }\end{array}$ \\
\hline $\begin{array}{l}\text { Financement en } \\
\text { fonction des } \\
\text { performances }\end{array}$ & Non & Oui & Oui \\
\hline $\begin{array}{l}\text { Suivi et évaluation du } \\
\text { programme }\end{array}$ & $\begin{array}{l}\text { Assurés principalement par les agents } \\
\text { d'exécution, le Secrétariat du Fonds } \\
\text { assurant une évaluation externe et } \\
\text { un rôle amplificateur. Supervisés } \\
\text { par le Conseil. }\end{array}$ & $\begin{array}{l}\text { Audits indépendants } \\
\text { des performances pour vérifier } \\
\text { les rapports vaccination. Principe } \\
\text { de non-intervention, le système } \\
\text { de financement étant basé sur } \\
\text { la performance. }\end{array}$ & $\begin{array}{l}\text { C'est le Conseil qui est responsable au } \\
\text { premier chef. Le mécanisme de } \\
\text { coordination au niveau national est un } \\
\text { acteur clé sur le plan local, les systèmes } \\
\text { existants sont utilisés dans la mesure } \\
\text { du possible. }\end{array}$ \\
\hline
\end{tabular}

Note : Adapté de : Heimans, J. (2002), Multisectoral Global Funds as Instruments for Financing Spending on Global Priorities, DESA Discussion Paper $n^{\circ} 24$, Département des affaires économiques et sociales des Nations unies, disponible sur: www.un.org/esa/esa02dp24.pdf.

\section{Notes}

1. Addison, T., M. McGillivray et M. Odedokum (2003), Donor Funding of Multilateral Agencies, Discussion Paper $n^{\circ}$ 2003/17, UNU/WIDER.

2. Jerve, A.M. et H. Selbervik (2003), MOPAN: Report from the 2003 Pilot Exercise: Chr. Michelsen Institute, Bergen.

3. DFID (2002), Working in Partnership with the World Health Organization, DFID, Londres.

4. Voir www.unhcr.ch, rubrique Contributions volontaires 2002.

5. Heimans, J. (2002), Multisectoral Global Funds as Instruments for Financing Spending on Global Priorities, DESA Discussion Paper $n^{\circ}$ 24, Département des affaires économiques et sociales des Nations unies, disponible sur : www.un.org/esa/esa02dp24.pdf. 


\section{Chapitre 11}

\section{Contrôles et contrepoids dans les systèmes de coopération au développement}

L'aide extérieure est une fonction particulière de l'État qui est souvent mal comprise et parfois considérée comme n'intéressant que les populations d'autres pays ou n'ayant qu'un impact limité. Cela la met vis-à-vis de l'opinion publique et du système politique national dans une position précaire et délicate. Il faut donc que les gestionnaires des programmes d'aide extérieure s'efforcent sans relâche d'informer le public de leurs activités et surtout de lui démontrer que l'aide est bien gérée et qu'elle produit des résultats. À la surprise de beaucoup de gens, les programmes d'aide extérieure sont soumis à divers systèmes rigoureux de contrepoids qui impliquent toute une gamme de contrôles. Mais les conclusions de ces contrôles sont généralement mal connues. Ces systèmes de contrepoids vont du suivi et de l'évaluation des activités des programmes à des systèmes de gestion axée sur les résultats, en passant par le contrôle de la Cour des comptes et les avis des organes consultatifs (informations, rétroactions et critiques). On peut donc penser que dans certains pays membres au moins les programmes de développement sont peut-être l'une des activités publiques les plus extensivement contrôlées. Quoi qu'il en soit, des mesures complémentaires sont en cours pour améliorer l'évaluation et le retour d'informations, dans le but à la fois d'en tirer des enseignements en interne et d’informer le public. 


\section{Suivi}

Le suivi de l'exécution des activités de développement fait partie intégrante du cycle des projets/programmes, et ceci en principe quel que soit le mode d'aide. L'OCDE/CAD en donne la définition suivante : "Processus continu de collecte systématique d'informations, selon des indicateurs choisis, pour fournir aux gestionnaires et aux parties prenantes d'une action de développement en cours des éléments sur les progrès réalisés, les objectifs atteints et l'utilisation des fonds alloués ${ }^{1}$. " Le suivi permet de faire le bilan des progrès réalisés et de proposer des actions correctives qui permettent d'atteindre les objectifs. Il incombe à la fois à l'agent d'exécution et à l'organisme de financement, mais doit également prendre en compte le point de vue des parties prenantes et des bénéficiaires. Pour assurer ce suivi, les cadres logiques, qui définissent des indicateurs et des moyens de vérification, constituent une bonne base.

Une action de développement bien conçue doit comporter des objectifs et des indicateurs clairs et mesurables (voir l'annexe A.5 qui traite de la gestion des projets et programmes de développement, et notamment de leur suivi). Les indicateurs mesurent l'exécution d'activités définies, l'obtention des résultats et la probabilité de réalisation des objectifs. Si ce n'est déjà fait, il peut être nécessaire dans la phase initiale du projet de procéder à un état des lieux qui servira de référentiel pour le suivi de l'avancement des travaux. Cet état des lieux décrit et analyse la situation, notamment socioéconomique, et son évolution sur une période donnée. Les données ainsi établies deviennent les points de référence par rapport auxquels on appréciera l'évolution de la situation et la réalisation des objectifs. Le suivi doit aussi prendre en compte les modifications de l'environnement externe et des principales hypothèses retenues comme base de l'activité.

La mission qui s'est rendue au Mozambique a constaté que le degré de suivi par les pays membres du CAD de l'impact de leurs activités est variable. Dans certains cas, en particulier ceux des pays où la gestion est centralisée, le suivi des activités est peu soumis à des règles expresses, ce qui est d'ailleurs parfois frustrant pour le personnel déployé sur le terrain. Parfois même, aucun cadre logique n'est établi ce qui signifie que le suivi se réduit à une collecte rudimentaire de données anecdotiques. Quelques pays en revanche ont mis en place des systèmes de gestion stratégique sophistiqués qui permettent de suivre de près les résultats des différentes activités puis de les agréger de façon à mesurer l'impact global du programme dans le pays d'accueil. Certains pays membres du CAD choisissent les indicateurs en fonction de chaque activité et des objectifs globaux du pays. D'autres s'efforcent délibérément d'impliquer au maximum le pays partenaire dans l'activité de suivi. Une autre tendance que l'on constate est l'utilisation croissante de l'informatique, lorsqu'il existe des systèmes de communication appropriés, pour suivre et évaluer toutes les activités financées par une agence de développement à travers le monde. 


\section{Évaluation}

Selon la définition de l'OCDE/CAD, l'évaluation est "l'appréciation, aussi systématique et objective que possible, d'un projet, d'un programme ou d'une politique en cours ou terminé, de sa conception, de sa mise en œuvre et de ses résultats. Le but est de déterminer la pertinence et l'accomplissement des objectifs, l'efficience en matière de développement, l'efficacité, l'impact et la durabilité. Une évaluation doit fournir des informations crédibles et utiles permettant d'intégrer les leçons de l'expérience dans le processus de décision des bénéficiaires et des bailleurs de fonds $»^{2}$. Les buts principaux des évaluations sont de fournir une base objective pour juger des performances de l'action, de fournir des informations sur les enseignements à tirer afin d'améliorer les actions futures, et d'assurer la transparence. Pour qu'elles répondent à ces objectifs, il faut qu'elles soient utilisées comme outils d'apprentissage et le cas échéant de modification des comportements au sein de l'Organisation. Plusieurs pays membres du CAD, notamment l'Australie ${ }^{3}$ et les États-Unis ${ }^{4}$, conscients à la fois de la nécessité et de l'intérêt d'une identification et d'un partage plus systématiques des enseignements de l'expérience, ont mis en place des systèmes de gestion par Internet de leur base de connaissances.

Les principaux aspects à prendre en compte dans l'évaluation des activités de développement sont les suivants :

- La pertinence de l'action par rapport à l'environnement considéré.

- L'impact, intentionnel ou non, de l'action et sa contribution à la réalisation de l'objectif global.

- L'efficacité de l'action par rapport à l'objectif fixé et la mesure dans laquelle la réalisation de cet objectif peut être attribuée à l'action en question.

- L'efficience de l'action, mesurée par les ressources utilisées par rapport aux résultats obtenus.

- La durabilité des résultats obtenus après la fin de l'aide extérieure.

Dans le cadre de son effort permanent d'amélioration de l'efficacité de l'aide, le CAD a adopté en 1991 un ensemble de Principes pour l'évaluation de l'aide au développement, qui ont fait l'objet d'une révision en 1998 et servent toujours de base aux examens par les pairs du CAD (voir encadré 11.1). Ceux-ci ont, ces dernières années, mis en évidence un certain nombre d'aspects qui ont encore besoin d'être améliorés :

- Un manque d'impartialité et d'indépendance des systèmes d'évaluation par rapport à la gestion opérationnelle.

- Des insuffisances au niveau de l'efficacité du suivi et de l'autoévaluation.

- Un déséquilibre entre l'apprentissage interne et l'obligation de transparence externe.

- Une attention insuffisante au retour d'information et à la diffusion des résultats, surtout dans les pays partenaires.

- Une implication insuffisante des bénéficiaires.

Par ailleurs, les examens par les pairs ont fait ressortir certains des principaux problèmes auxquels sont confrontés les pays membres en matière d'évaluation. Ce sont:

- La difficulté de se comporter en organisation apprenante, de promouvoir une culture de l'évaluation et du résultat, et d'instaurer des liens systémiques entre les missions locales et les services centraux ainsi que les divers acteurs institutionnels. 
- La nécessité d'établir des liens stratégiques entre le suivi et l'évaluation destinés à l'apprentissage interne et l'évaluation indépendante destinée à assurer la transparence vis-à-vis de l'extérieur.

- La nécessité d'aller au-delà de l'analyse des projets et d'effectuer des évaluations par secteur et par pays et des évaluations de processus.

- La nécessité d'élaborer des outils méthodologiques appropriés pour évaluer les stratégies de lutte contre la pauvreté (SLP) et l'aide-programme sectorielle afin que les informations fournies soient utiles pour l'action et la décision.

- La nécessité de renforcer le rôle des collectivités et organisations de pauvres, ainsi que les pauvres eux-mêmes, dans les systèmes de suivi et d'évaluation.

\section{Encadré 11.1. Principes du CAD pour l'évaluation de l'aide au développement}

Les principes du CAD englobent les éléments essentiels suivants :

- Les organismes d'aide doivent avoir une politique d'évaluation qui établit des lignes directrices et des méthodes claires et définit de façon précise le rôle et les missions de la fonction d'évaluation et sa place dans la structure institutionnelle

- Le processus d'évaluation doit être impartial et indépendant des mécanismes mis en place pour l'élaboration de la politique d'aide, et l'acheminement et la gestion des concours.

- L'exercice d'évaluation doit être aussi ouvert que possible et ses résultats largement diffusés.

- Les évaluations ne sont utiles que si elles sont exploitées. Il est donc essentiel que leurs résultats parviennent aussi bien aux décideurs qu'aux agents opérationnels.

- Le partenariat avec les pays d'accueil et la coopération entre donneurs sont tous deux indispensables à l'évaluation de l'aide ; ce sont des aspects importants de l'édification des capacités institutionnelles du pays bénéficiaire et de la coordination de l'aide, qui sont susceptibles d'alléger le fardeau administratif qui pèse sur les pays d'accueil.

- L'évaluation de l'aide et les exigences qui l'accompagnent doivent être intégrées dès le départ dans la planification de l'aide. Une définition claire des objectifs de l'activité d'aide est indispensable à l'efficacité de l'évaluation par la suite.

Comme le soulignent les principes du CAD, l'indépendance et la transparence sont essentielles au bon fonctionnement des systèmes d'évaluation. À cet égard, les pays membres du CAD tendent à adopter des approches diverses, qui sont notamment fonction de leur contexte national (voir plus loin).

\section{Un exemple : le Japon}

Pour le Japon, la transparence et la crédibilité de la performance de son APD sont importantes. Chaque institution d'aide possède son propre comité d'évaluation, composé de spécialistes extérieurs, qui examine la qualité et la méthodologie de chaque évaluation. Tous les comptes rendus d'évaluation sont rendus publics, et la plupart sont disponibles en ligne. Un rapport annuel d'évaluation ${ }^{5}$ est par ailleurs adressé à la Diète (Parlement) par le ministère des Affaires étrangères, la JICA et la JIBC. La Cour des Comptes possède aussi une section administrative spécialement dédiée au suivi de l'utilisation de l'APD, et les audits externes s'étendent désormais à toutes les modalités de l'aide. 
Les évaluations peuvent être effectuées à divers niveaux et selon diverses modalités. Les projets ou activités peuvent être évalués individuellement en cours de route ou après achèvement. Cependant, les donneurs tendent aussi à conduire des évaluations à un niveau plus global, qui couvrent soit des activités du même type menées dans plusieurs pays partenaires, soit un éventail d'activités menées dans le même pays partenaire. D'autre part, des évaluations conjointes interviennent de plus en plus régulièrement ; elles constituent en effet le moyen logique d'évaluer l'impact de la contribution collective des donneurs à des mécanismes de financement commun tels que le soutien au budget général. Le Réseau du CAD pour l'évaluation du développement a établi des directives sur la manière d'organiser les évaluations conjointes des programmes de développement ${ }^{6}$.

Cette progression des évaluations globales et conjointes témoigne d'une volonté d'évaluer l'impact global des programmes de développement sur l'avancée d'un pays partenaire ou d'un secteur vers les Objectifs du millénaire pour le développement. L'importance grandissante que les pays membres du CAD y attachent est en partie liée à la montée en puissance du système des partenariats et de l'alignement sur les stratégies nationales de lutte contre la pauvreté, qui rend difficile d'évaluer la contribution d'un donneur séparément de celles des autres organismes bilatéraux et multilatéraux. Cette évaluation de l'impact global des programmes d'aide peut nécessiter la mise en place, à partir des évaluations conjointes déjà entreprises, d'un processus collectif plus intégré qui devrait en principe être piloté par les pays partenaires, moyennant un soutien accru au renforcement de leurs capacités de suivi et d'évaluation des résultats. Ce type de processus s'impose si l'on veut fournir aux Parlements, à l'opinion publique et aux décideurs une information fiable sur l'efficacité de l'ensemble des efforts déployés pour progresser sur la voie des Objectifs du millénaire pour le développement.

\section{Tableau 11.1. Principes appliqués par Danida pour adapter à différents publics} les vecteurs du retour d'information

\begin{tabular}{|c|c|c|c|}
\hline \multirow[b]{2}{*}{ But principal } & \multicolumn{3}{|c|}{ CATÉGORIES DE PUBLICS } \\
\hline & Reddition de comptes & Apprentissage & Partenaires \\
\hline VECTEURS & $\begin{array}{l}\text { Parlementaires/leaders } \\
\text { d'opinion/publics }\end{array}$ & $\begin{array}{l}\text { Universitaires, étudiants, } \\
\text { chercheurs } \\
\text { Base de ressources extérieure } \\
\text { (consultants, etc.) } \\
\text { ONG }\end{array}$ & $\begin{array}{c}\text { Partenaires du pays en } \\
\text { développement } \\
\text { Autres organismes } \\
\text { de développement }\end{array}$ \\
\hline Rapport d'évaluation & & - & - \\
\hline Synthèse (4 pages) & $\bullet$ & $\bullet$ & $\bullet$ \\
\hline Version populaire (25 pages) & - & & \\
\hline Communiqué de presse & $\bullet$ & & \\
\hline Vidéo/film & - & & - \\
\hline Rapport annuel au Conseil & $\bullet$ & & \\
\hline Rapport annuel de Danida & $\bullet$ & & \\
\hline Bulletin d'information Danid@visen & - & & \\
\hline Réunions publiques et organisations professionnelles & & $\bullet$ & \\
\hline Conférences dans les lycées et universités & & $\bullet$ & \\
\hline Web & - & $\bullet$ & - \\
\hline Séminaires/ateliers & & & $\bullet$ \\
\hline Participation à des évaluations & & & - \\
\hline
\end{tabular}

Source : Danida (2000), External Feedback - Danida's Dissemination Vehicles, document présenté à l'Atelier de Tokyo sur l'exploitation des résultats des évaluations pour mieux apprendre et rendre compte, septembre 2000. 
Les mécanismes de retour de l'information constituent un volet important de l'évaluation et les pays membres du CAD sont conscients de la nécessité d'améliorer et développer leurs pratiques en la matière ${ }^{7}$. Or, ce retour d'information intéresse à des titres divers de nombreux publics différents qui impliquent des approches différentes. Il faudra éventuellement sélectionner les publics qui présentent le plus d'importance pour chaque agence, et les méthodes à utiliser pour s'adresser à eux. L'exemple de Danida ${ }^{8}$ montre comment on peut adapter le retour d'information à des publics divers (voir tableau 11.1).

\section{Gestion axée sur les résultats en termes de développement}

Dans le cadre de l'effort général entrepris par la communauté des donneurs pour améliorer l'efficacité de l'aide, plusieurs pays membres mettent de plus en plus l'accent sur la mesure des résultats et de l'impact de leurs activités en termes de développement, et cherchent à déterminer jusqu'à quel point les résultats en question peuvent être imputés à des activités données. Ce processus est souvent appelé gestion axée sur les résultats, ou gestion des performances.

Le Forum des partenaires au développement organisé par le CAD en 2002 a réuni donneurs et partenaires sur le thème de la gestion axée sur les résultats en termes de développement, et de l'efficacité de l'aide ${ }^{9}$. Les discussions ont montré la nécessité de donner plus d'importance à l'évaluation des résultats des programmes d'aide et à la mise en place de systèmes axés sur les résultats.

La deuxième Table ronde internationale sur la gestion au service des résultats en termes de développement, qui a réuni à Marrakech en février 2004 les membres du CAD et les organisations multilatérales, a défini les résultats comme étant des " améliorations durables de la situation du pays en cause " et la gestion axée sur les résultats en termes de développement comme une "stratégie de gestion axée sur la performance et sur la concrétisation de produits, de résultats et d'impacts ${ }^{10}$. Les cinq grands principes retenus par la Table ronde sont les suivants ${ }^{11}$ :

- À tous les stades - de la planification stratégique à l'exécution et à l'achèvement et au-delà - centrer le dialogue sur les résultats pour les pays partenaires, les organismes de développement et les autres parties prenantes.

- Axer les activités effectives de programmation, de suivi et d'évaluation sur les résultats recherchés, tels qu'ils ont été fixés.

- Maintenir le système de notification des résultats aussi simple, clair et efficace par rapport à son coût que possible.

- Gérer en vue des résultats, et non sur la base des résultats déjà acquis.

- Tirer profit des informations relatives aux résultats pour améliorer la gestion et pour la prise des décisions ainsi que pour la notification et la reddition de comptes.

Les pays membres du CAD, parmi lesquels le Royaume-Uni et les États-Unis, appliquent de plus en plus à leurs programmes de développement la gestion axée sur les résultats (voir ci-dessous). 


\section{Un exemple : Les États-Unis}

Le système de gestion des performances adopté par l'USAID est fondé sur la définition des objectifs stratégiques (SO), qui sont le plus haut niveau de résultats sur lequel une unité opérationnelle de l'USAID et ses partenaires peuvent avoir une influence, compte tenu du temps et des moyens dont ils disposent. Chaque unité opérationnelle a son plan de gestion des performances qui précise des objectifs de performance annuels et de long terme. Au Mozambique, le cadre de résultats de l'USAID comprend quatre objectifs stratégiques, qui comportent chacun un certain nombre de résultats intermédiaires et sous-intermédiaires, avec les indicateurs correspondants. Par exemple, l'objectif stratégique d'une " augmentation des revenus des ménages ruraux dans les zones cibles » comporte un résultat intermédiaire: " amélioration de l'accès aux marchés " et quatre résultats sous-intermédiaires: i) "amélioration du contexte des activités marchandes "; ii) "réhabilitation et entretien des routes "; iii) " accroissement de la capacité de commercialisation et de transport » et iv) "information sur les marchés et système opérationnel de commercialisation des produits ».

Un exemple : Le Royaume-Uni

Dans la fonction publique britannique, la gestion axée sur les résultats s'articule autour du Public Service Agreement (PSA) et du Service Delivery Agreement (SDA) qui l'accompagne. Comme pour les autres ministères, le PSA fixe pour le DFID les objectifs qu'il doit remplir avec les moyens dont il est doté pour la période 2003-2006. Ces objectifs correspondent à des résultats intermédiaires qui sont directement liés aux Objectifs du millénaire pour le développement. Les progrès réalisés sont mesurés par des indicateurs supplétifs comme la mortalité infantile et la santé maternelle, dont on considère que la progression donne une idée assez bonne des avancées d'ensemble du pays considéré. Le SDA qui sous-tend le PSA définit ce que le DFID doit faire pour contribuer aux résultats demandés par le PSA dans cette période. Les objectifs du SDA sont constitués de produits intermédiaires, d'activités et de processus sur lesquels le DFID a plus de pouvoir que sur les objectifs de résultats du PSA. Des plans triennaux de performance établis pour chaque Direction du DFID définissent l'échelle de contribution de la réalisation des objectifs du SDA aux résultats du PSA. À l'intérieur de ces plans triennaux, on trouve des plans annuels par département et par guichet pays ainsi que des plans de travail et des objectifs par équipe, qui eux-mêmes se décomposent en plans de performances individuelles pour chaque agent (voir ci-dessous).

Les pays membres du CAD ont mis en évidence quelques-uns des défis que posent la mise en place et le fonctionnement de la gestion axée sur les résultats dans le domaine du développement.

- Défis organisationnels : motivation des agents de l'Organisation; changement de culture de l'Organisation; insuffisance de capacité, notamment sur le plan des compétences ; nécessité de moyens supplémentaires pour financer la mise en place de la capacité voulue; difficulté de fixer des objectifs cohérents et intégrés partagés par tous les organismes qui participent à un programme ; capacité d'élaborer des systèmes simples, souples et adaptables ; et nécessité de directives plus précises sur l'application du système de gestion axée sur les résultats dans le cas d'initiatives multidonneurs telles que les programmes sectoriels. 


\section{Graphique 11.1. Du niveau des OMD au niveau indviduel : stratégie et organisation du DFID}

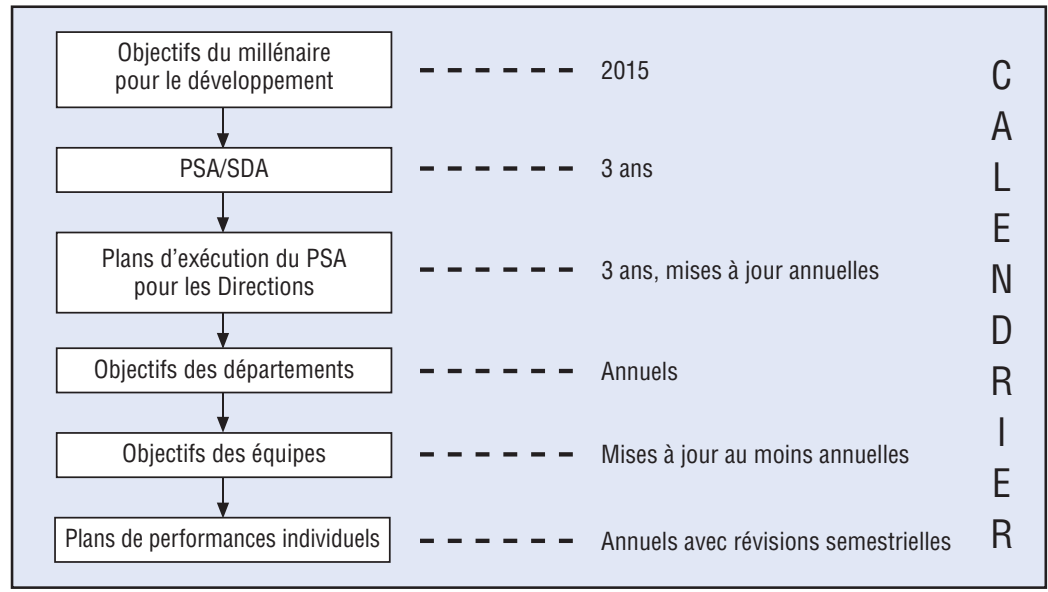

- Difficultés de mesure : détermination d'indicateurs mesurables; collecte d'un trop grand nombre d'informations et difficultés de gestion qui en résultent ; enfin difficulté de mesurer les résultats.

- Difficultés d'attribution : difficulté de définir la contribution d'un organisme ; partage de la responsabilité de réalisation des résultats intermédiaires avec les autres donneurs ; identification des impacts respectifs.

- Problèmes des pays partenaires : manque de capacité des systèmes partenaires en matière de collecte des informations de performance; objectifs de la SLP ou autres objectifs nationaux trop ambitieux; nécessité d'harmoniser les exigences en matière d'information afin de réduire la charge pour les systèmes des pays partenaires.

Le CAD aide les donneurs à surmonter ces difficultés en leur offrant, au travers de son groupe de travail sur l'efficacité de l'aide et les pratiques des donneurs, une plate-forme d'échanges et d'apprentissage mutuel sur les nouvelles pratiques, en intégrant la gestion axée sur les résultats au programme général d'action des membres du CAD et de leurs partenaires, et en définissant, dans le cadre des principes et des orientations générales définis par le CAD, des valeurs, des approches méthodologiques et des procédures de coopération communes.

\section{Intervention des instances nationales de contrôle des comptes}

Les instances nationales de contrôle des comptes des pays membres du CAD sont des organismes indépendants qui émettent des avis objectifs et publics sur différents aspects du fonctionnement de l'État. Dans de nombreux pays membres, l'autorité de contrôle des comptes se penche de plus en plus sur le fonctionnement du programme de développement. Les rapports qu'elle publie peuvent se borner à juger du respect des pratiques et procédures de l'État (audit de conformité), y compris dans la manière dont est dépensé l'argent du contribuable, ou s'étendre à l'efficience des dépenses et aux résultats obtenus (audit de performance). Dans ce dernier cas, le rôle de l'autorité de contrôle des comptes vient compléter la fonction d'évaluation des organismes en charge du développement.

Certains pays membres du CAD, fournissant désormais des volumes importants de ressources aux pays partenaires sous forme de soutien sectoriel ou d'aide programme, ont constaté la nécessité de travailler plus étroitement avec leur autorité nationale de contrôle 
des comptes pour lui faire mieux connaître les objectifs de l'aide au développement et ses différentes modalités (voir plus loin). À cet égard, le Comptroller et Auditor-General du Royaume-Uni, par exemple, a souligné l'importance de l'efficience de l'aide et de son impact sur le développement, tout en reconnaissant que dans le domaine du développement la gestion et la mesure des performances soulèvent quelques difficultés, le temps nécessaire pour voir apparaître des résultats concrets étant souvent plus long que les délais impartis pour le suivi et la notification des dépenses publiques. À cela s'ajoutent des difficultés pour mesurer et attribuer les impacts et pour obtenir des données fiables ${ }^{12}$.

\section{Un exemple : le Canada}

L'ACDI procède périodiquement à des audits de ses programmes en vue de s'assurer de l'utilisation efficace et efficiente de ses ressources. Pour mieux intégrer la fonction d'audit dans le processus global d'évaluation des performances, le contrôle interne a été recentré sur les meilleures pratiques, et non plus sur la simple conformité aux règles. De plus, des examens périodiques indépendants sont effectués par le Bureau du vérificateur général (BUG) afin de fournir au Parlement des informations et avis objectifs. Une collaboration très constructive s'est instaurée au fil des ans entre l'ACDI et le BUG. Celui-ci a par exemple demandé à l'ACDI de dresser à l'intention du public un tableau plus clair et plus équilibré de ses performances. Plus récemment, le BUG a suivi de près les débats au sein de l'ACDI sur l'efficacité de l'aide et approuvé le recentrage sur des programmes, à condition que l'on puisse mesurer les résultats en termes de développement et mettre en place des mécanismes appropriés d'évaluation financière et de compte rendu.

La mission effectuée au Mozambique a permis de constater que les autorités nationales de contrôle des comptes des pays membres du CAD s'intéressent davantage aux activités que financent les donneurs. Cela dit, les missions des contrôleurs nationaux au Mozambique ont eu tendance à manquer de coordination, et il reste beaucoup à faire pour mieux harmoniser ce type d'activités, notamment sur le plan des normes utilisées, comme cela se fait actuellement pour les évaluations. Un groupe de sept pays membres du CAD Danemark, Finlande, Irlande, Pays-Bas, Norvège, Suède et Royaume-Uni - dénommé le "Groupe nordique + ", a entrepris une action d'harmonisation des normes dans les domaines des exigences financières, procédurales et légales ${ }^{13}$. Cela pourra consister à se mettre d'accord sur des cadres juridiques pour la mise en commun des fonds et sur des normes de contrôle et de notification mutuellement acceptables. Au bout du compte, la reconnaissance et l'exploitation par les instances nationales de contrôle des comptes des audits effectués dans les pays d'accueil par leurs homologues des autres pays membres pourraient alléger considérablement la charge administrative qui pèse sur les pays partenaires. Les progrès réalisés dans ce sens sont lents, mais le fait même que l'on envisage cette possibilité montre la volonté de nombreux donneurs d'explorer de nouveaux moyens de travailler ensemble en partenariat.

\section{Organismes consultatifs}

La moitié environ des pays membres du CAD ont mis en place un comité consultatif auprès du ministre ou de l'administration qui a la charge principale du développement. La structure, la fonction, la composition et le rôle de ces comités divergent, mais en général ils se distinguent des organes consultatifs habituels en ce sens qu'ils ont un rôle de conseil 
expressément défini et une plus grande autonomie. Quelques pays membres possèdent plusieurs organes consultatifs.

La composition et la taille des organes consultatifs varient beaucoup et peuvent dépendre des fonctions qu'ils ont à remplir. Certains comprennent des spécialistes ou experts de diverses branches, tandis que d'autres comprennent une représentation plus large de la société civile, notamment des ONG spécialisées dans le développement ou des universitaires. Un certain nombre d'organes consultatifs comprennent, à côté de représentants de la société civile, d'anciens ministres ou représentants du gouvernement (voir plus loin). Leur taille peut varier de 9 à 50 membres.

\section{Un exemple : la Suisse}

La Commission consultative suisse pour le développement et la coopération internationaux comprend des représentants des partis représentés au Parlement, de la société civile - syndicats, ONG, universités et médias - ainsi que du secteur privé. Elle a pour mission de conseiller le Conseil fédéral sur la coopération au développement et l'aide humanitaire. Elle a créé au moins trois sous-commissions pour suivre de plus près les institutions et activités présentant un intérêt particulier : les institutions de Bretton Woods, l'OMC et la coopération avec les pays d'Europe orientale. Elle constitue un forum de discussion unique en son genre qui facilite le dialogue entre les pouvoirs publics et la société civile et joue un rôle considérable pour assurer le soutien de la population suisse à la coopération au développement et à la politique suisse dans ce domaine.

Certains organes consultatifs ont plusieurs fonctions, d'autres ont des rôles plus circonscrits. Les fonctions en question peuvent comporter l'examen des grandes orientations de la politique de développement et des tâches plus spécifiques telles que l'examen des stratégies par pays, celui des propositions d'aide, la commande ou l'approbation d'études et l'établissement de documents de planification. Un certain nombre de ces organes contribuent à l'évaluation des programmes, soit en examinant les rapports d'audit et d'évaluation, soit en participant activement à l'évaluation elle-même. Certains sont chargés d'organiser des forums ou un dialogue sur le développement entre la société civile et les pouvoirs publics.

L'efficacité des organes consultatifs dépend en partie de leur structure et des rôles qui leur sont confiés. Une composition large, par exemple, peut susciter des difficultés de consensus ou des ambiguïtés, tandis que la présence de membres d'un gouvernement en place peut nuire à l'autonomie de l'organe en cause. Les organes consultatifs doivent en outre, pour être efficaces, être dotés de moyens suffisants en termes de temps consacré par le personnel de l'administration du développement à la gestion et à la consultation, ainsi que de temps et de frais de mise à disposition de leurs membres. Dans certains pays membres du $\mathrm{CAD}$, les organes consultatifs fonctionnent exclusivement à titre bénévole, mais jouent néanmoins un rôle important dans le programme de coopération au développement.

\section{Un exemple : la France}

Le Haut Conseil de la coopération internationale créé en 1999 est une instance consultative placée auprès du Premier ministre, dont le but est d'impliquer la société civile dans la politique française du développement en offrant une tribune aux acteurs non gouvernementaux. Il a pour mission de favoriser une concertation régulière entre les différents acteurs de la coopération au 
développement et de sensibiliser l'opinion publique aux enjeux de cette coopération. Il est composé de 45 membres nommés pour 3 ans, qui comprennent des représentants d'ONG et d'autres organisations de la société civile, des élus des collectivités territoriales qui interviennent dans la coopération décentralisée, ainsi que des parlementaires. Il est doté d'un budget propre et d'un secrétariat d'une dizaine de personnes. Son travail s'organise autour d'un certain nombre de groupes de travail thématiques. Ses principales activités sont l'organisation de conférences annuelles sur des questions de développement ainsi que l'établissement de rapports et avis, parfois en collaboration avec des commissions parlementaires ou à la demande du gouvernement. Son indépendance est attestée par le fait qu'il ne comprend aucun membre du gouvernement.

\section{Notes}

1. OCDE (2002), Glossaire des principaux termes relatifs à l'évaluation et la gestion axées sur les résultats, OCDE, Paris.

2. OCDE (2002), Glossaire des principaux termes relatifs à l'évaluation et la gestion axées sur les résultats, OCDE, Paris.

3. AusAID's Knowledge Warehouse (AKWa) http://akwa.ausaid.gov.au/ comporte les enseignements tirés de la mise en œuvre du programme d'aide australien, des exemples de bonnes pratiques sur la totalité du cycle d'activités et une série de publications de l'AusAID relatives à l'assurance de qualité et à l'évaluation des projets et programmes d'aide de l'Australie.

4. Pour améliorer la diffusion des enseignements de l'expérience, l'USAID a créé un site intitulé « Knowledge for Development » dont l'adresse est : http://knowledge.usaid.gou/index.html.

5. Les rapports annuels d'évaluation de la coopération économique du Japon sont disponibles sur: www.infojapan.org/policy/oda/evaluation/.

6. OCDE (2000), Effective Practices in Conducting a Joint Multi-Donor Evaluation, OCDE, Paris.

7. OCDE (2001), Evaluation Feedback for Effective Learning and Accountability, OCDE, Paris.

8. Le ministère danois des Affaires étrangères utilise couramment l'appellation "Danida " pour désigner la coopération danoise au développement, qui représente $90 \%$ du programme d'activités du département Sud du ministère des Affaires étrangères.

9. OCDE (2003), "Pour une gestion axée sur les résultats du développement et sur l'efficacité de l'aide : rapport sur le Forum des partenaires au développement organisé par le CAD », les dossiers du CAD, volume 4, $\mathrm{n}^{\circ}$ 3, OCDE, Paris, pp. 47-76.

10. Action Plan on Managing for Development Results. Consultable à l'adresse : www.mfdr.org/documents/ 3MarrakechActionPlan05febA4.pdf.

11. Promoting a Harmonized Approach to Managing for Development Results: Core Principles. Consultable à l'adresse : www.mfdr.org/documents/2CorePrinciples05Feb04.pdf.

12. Comptroller and Auditor General (2002), Performance Management - Helping to Reduce Poverty: Report by the Comptroller and Auditor General HC 739 Session 2001-2002, HMSO, Londres.

13. Joint Action Plan for Effective Aid Delivery through Harmonisation and Alignment of Donor Practices 2003-2005 (la Danemark, le Finlande, l'Irlande, la Norvège, les Pays Bays, la Royaume Unit et la Suède) consultable à : www.aidharmonization.org/download/236284/NordicPlus.pdf. 



\section{ANNEXE A.1 \\ Profils de base des programmes d'aide extérieure des pays membres du CAD}

Vous trouverez sur www.oecd.org/dac/stats toutes les données relatives aux graphiques contenus dans cette publication sauf mention contraire. 


\section{Allemagne}

Objectif :

Lutter contre la pauvreté dans le monde, préserver la paix, rendre la mondialisation équitable et durable. Le programme allemand de coopération pour le développement contribue aux efforts menés conjointement à l'échelle internationale pour atteindre les OMD

(Programme d'action à l'horizon 2015 à l'appui de la lutte contre la pauvreté - 2001).

Législation:

Sans objet.

Déclaration de politique Programme d'action à l'horizon 2015 à l'appui de la lutte contre la pauvreté - Contribution du gouvernement allemand visant à réduire générale sur l'aide extérieure : de moitié la grande pauvreté à l'échelle mondiale (2001).

Autres déclarations de politique:

Onzième rapport d'orientation du gouvernement allemand sur le développement (2001) ;

Ministre responsable

Autres ministres:

Déclaration de coalition (Government's Coalition Statement) (2002).

Ministre fédéral de la Coopération et du Développement économique.

Ministre fédéral des Affaires étrangères.

Ministre fédéral des Finances.

Ministère/organisme de

développement principal :

Ministère fédéral de la Coopération et du Développement économique (BMZ) (www.bmz.de).

Autres organismes/ministères: Agence de coopération technique (GTZ) (www.gtz.de).

Banque pour le développement (KfW) (www.kfw.de).

Ministère fédéral des Affaires étrangères (www.auswaertiges-amt.de/www/en/index_htm!).

German Investment and Development Corporation (DEG) (www.deginvest.de).

Service allemand du développement (DED) (www.ded.de).

InWEnt - Capacity Building International (www.inwent.org).

Structures de coordination

Le BMZ coordonne la coopération pour le développement avec les autres ministères.

interministérielles :

Principaux partenaires

bilatéraux:

Principaux domaines de

coopération :

Le BMZ met l'accent sur 40 pays prioritaires et 35 pays partenaires.

Les objectifs sectoriels concernent les domaines suivants : VIH/sida, enseignement fondamental, forêts humides, énergies renouvelables, efficacité énergétique, approvisionnement en eau et assainissement, construction de la paix et prévention des conflits. Autres domaines jugés prioritaires en accord avec les partenaires : réforme économique, démocratie, société civile, avis publics, approvisionnement en eau et assainissement, protection de l'environnement, santé, planification familiale et VIH/sida.

Organes ministériels Conseil consultatif auprès du ministère.

consultatifs :

\section{ALLEMAGNE}

\section{APD nette}

Prix courants (USD m)

En euros (millions)

APD/RNB

Aide bilatérale (\%)

Aide publique nette (AP)

Prix courants (USD m)

\section{Dix principaux bénéficiaires d'APD/AP brute (USD m)}

1 Serbie \& Monténégro

2 Chine

3 Congo, Rép. dém.

4 Cameroun

5 Bolivie

6 Inde

7 Zambie

8 Mozambique

9 Indonésie

10 Turquie
APD bilatérale brute, moyenne 2002-03, sauf indication contraire

Par groupe de revenu (USD m)

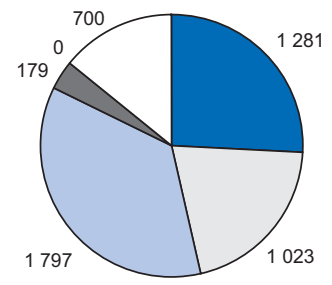

口Pays moins avancés $\square$ Faible revenu

$\square$ Revenu intermédiaire, tranche inférieure $\square$ Revenu intermédiaire, tranche supérieure $\square$ Revenu éleve

$\square$ Non ventilé

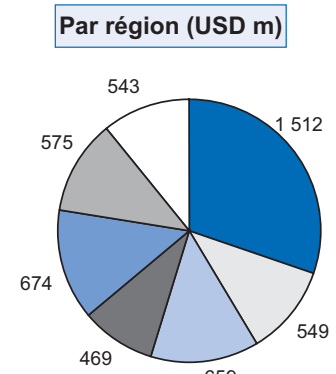

324

305

285

224

212

159

139

134

120 115

Afrique subsaharienne

$\square$ Asie du Sud et centrale

$\square$ Autres pays d'Asie \&

Océanie

Moyen-Orient et Afrique

du Nord

$\square$ Amérique latine \&

Amerique
Caraïbes

$\square$ Europe

$\square$ Non spécifié

\begin{tabular}{|l|l|l|l|l|l|l|} 
& & & & & \\
\hline
\end{tabular}




\section{Australie}

Objectif :

Servir l'intérêt national en aidant les pays en développement à lutter contre la pauvreté et à mettre en place un développement durable (Better Aid for a Better Future 1997).

Législation :

Sans objet.

Déclaration de politique Better Aid for a Better Future (1997).

générale sur l'aide extérieure :

Autres déclarations de Australian Aid: Investing in Growth, Stability and Prosperity (2002).

politique :

Reducing Poverty - the Central Influencing Factor of Australia's Aid Programme (2001).

Ministre responsable :

Autres ministres:

Ministre des Affaires étrangères, assisté d'un Secrétaire parlementaire.

Ministre des Finances.

Ministère/organisme de

développement principal :

Autres organismes/

ministères:

Agence australienne de développement international (AusAID) (www.ausaid.gov.au).

Ministère des Finances (www.treasury.gov.au).

Centre australien de la recherche agricole internationale (ACIAR) (www.aciar.gov.au).

Structures de coordination

interministérielles :

Principaux partenaires

bilatéraux :

La Papouasie Nouvelle-Guinée est le principal partenaire bilatéral. Le programme d'aide est axé sur le Pacifique et l'Asie orientale, et essentiellement sur le Myanmar, le Cambodge, la Chine, Fidji, l'Indonésie, Kiribati, le Laos, la Mongolie, les Philippines, le Samoa, les îles Salomon, la Thaïlande, le Timor oriental, les Tonga, Tuvalu, Vanuatu et le Viêt-nam.

Principaux domaines de Gouvernance, santé, éducation, développement agricole et rural, infrastructure.

coopération :

Organes ministériels

consultatifs :

Conseil consultatif pour les programmes d'aide.

AUSTRALIE
\begin{tabular}{|lrrc|}
\hline APD nette & $\mathbf{2 0 0 2}$ & $\mathbf{2 0 0 3}$ & Variation \\
Prix courants (USD m) & 989 & 1219 & $23.2 \%$ \\
Prix constants (2002 USD m) & 989 & 993 & $0.4 \%$ \\
En dollars austr. (millions) & 1821 & 1878 & $3.2 \%$ \\
APD/RNB & $0.26 \%$ & $0.25 \%$ & \\
Aide bilatérale (\%) & $78 \%$ & $80 \%$ & \\
Aide publique nette (AP) & & & \\
Prix courants (USD m) & 7 & 9 & $18.0 \%$ \\
\hline
\end{tabular}

APD bilatérale brute, moyenne 2002-03, sauf indication contraire

\begin{tabular}{|lr|}
\hline \multicolumn{2}{|c|}{ Dix principaux bénéficiaires } \\
d'APD/AP brute (USD m) \\
1 Papouasie-Nlle-Guinée & 195 \\
2 Indonésie & 79 \\
3 Salomon, lles & 44 \\
4 Viêt-nam & 38 \\
5 Timor-Leste & 33 \\
6 Philippines & 32 \\
7 Chine & 29 \\
8 Cambodge & 21 \\
9 Irak & 21 \\
10 Bangladesh & 17 \\
\hline
\end{tabular}

17 Par groupe de revenu (USD m)

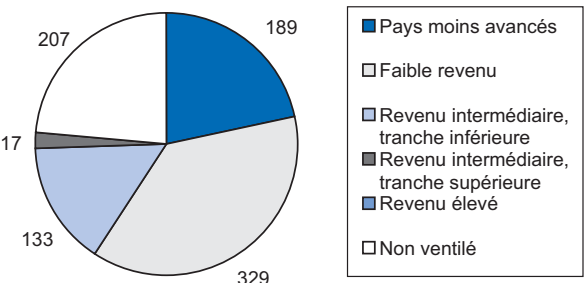

Par région (USD $\mathrm{m}$ )

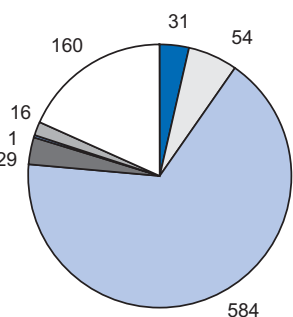

$\square$ Afrique subsaharienne $\square$ Asie du Sud et centrale $\square$ Autres pays d'Asie \& Océanie Moyen-Orient et Afrique du Nord $\square$ Amérique latine \&

Caraïbes 口Europe

$\square$ Non spécifié

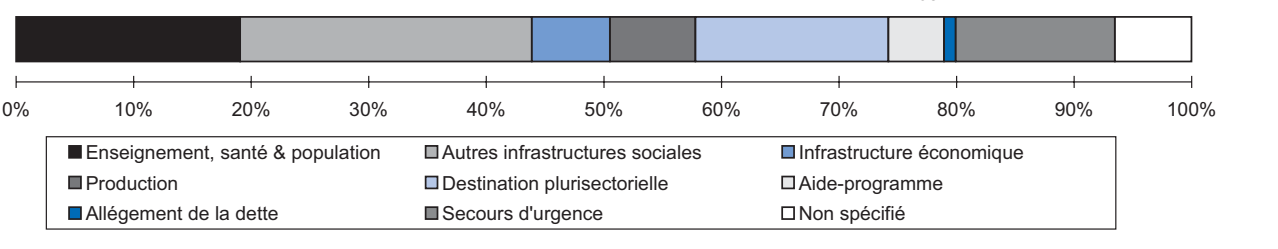




\section{Autriche}

Objectif :

Législation:

Déclaration de politique générale sur l'aide extérieure

Autres déclarations de politique :

Ministre responsable Autres ministres :

Ministère/organisme de développement principal :

Autres organismes/ ministères :

Structures de coordination interministérielles :

Principaux partenaires bilatéraux :

Principaux domaines de coopération :

Organes ministériels consultatifs :
Lutter contre la pauvreté par le développement économique et social, assurer la paix et la sécurité, préserver l'environnement et protéger les ressources naturelles (Federal Act on Development Co-operation 2002).

Federal Act on Development Co-operation (2002, modifié en 2003).

Programme triennal sur la politique autrichienne en matière de développement (adapté chaque année).

Ministre fédéral des Affaires étrangères.

Ministère des Affaires étrangères (Direction de la coopération pour le développement et de la coopération avec l'Europe orientale) (www.eza.gv.at)

Agence autrichienne de développement (ADA) (www.ada.gv.at).

Ministère fédéral des Finances.

Ministère fédéral de l'Éducation, de la Science et de la Recherche.

Ministère fédéral de l'Intérieur.

Provinces.

Huit pays sont « prioritaires » dans le cadre du programme autrichien de coopération pour le développement. En Afrique : le Burkina Faso, le Cap-Vert, l'Éthiopie, le Mozambique, le Rwanda et l'Ouganda ; en Amérique centrale : le Nicaragua ; en Asie : le Bhoutan. On dénombre également douze «pays de coopération ». En Afrique : le Burundi, le Kenya, la Namibie, le Sénégal, l'Afrique du Sud, la Tanzanie et le Zimbabwe ; en Asie : le Népal et le Pakistan ; en Amérique centrale : le Costa Rica, le Guatemala et El Salvador ; il faut ajouter également les Zones sous administration palestinienne, qui font l'objet d'un programme spécial.

Éducation, énergie, développement rural, soutien aux micro-, petites et moyennes entreprises, transport et mobilité, approvisionnement en eau et assainissement.

Commission consultative sur la politique de développement

\section{AUTRICHE}

\begin{tabular}{|lrrc|}
\hline APD nette & $\mathbf{2 0 0 2}$ & $\mathbf{2 0 0 3}$ & Variation \\
Prix courants (USD m) & 520 & 505 & $-3.0 \%$ \\
Prix constants (2002 USD m) & 520 & 414 & $-20.5 \%$ \\
En euros (millions) & 552 & 447 & $-19.1 \%$ \\
APD/RNB & $0.26 \%$ & $0.20 \%$ & \\
Aide bilatérale (\%) & $70 \%$ & $45 \%$ & \\
Aide publique nette (AP) & & & \\
Prix courants (USD m) & 196 & 245 & $25.1 \%$ \\
\hline
\end{tabular}

\begin{tabular}{|lr|}
\hline \multicolumn{2}{|c|}{$\begin{array}{c}\text { Dix principaux bénéficiaires } \\
\text { d'APD/AP brute (USD m) }\end{array}$} \\
1 Pologne (AP) & 93 \\
2 Serbie \& Monténégro & 53 \\
3 Égypte & 19 \\
4 Tanzanie & 17 \\
5 Turquie & 16 \\
6 Bosnie-Herzégovine & 13 \\
7 Mozambique & 12 \\
8 Russie (AP) & 12 \\
9 Bulgarie (AP) & 10 \\
10 Afghanistan & 10 \\
\hline
\end{tabular}

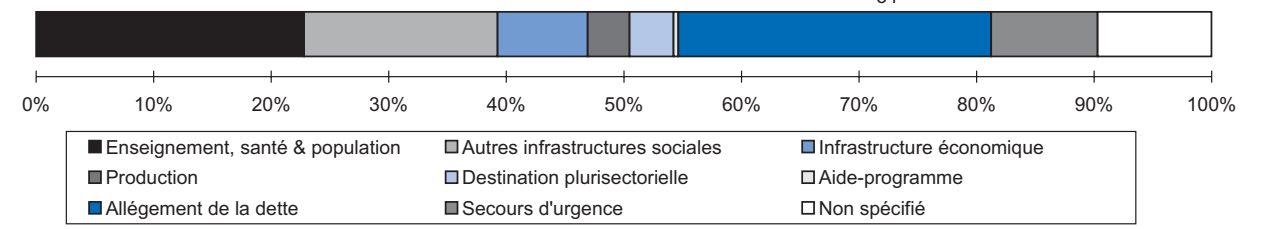

APD bilatérale brute, moyenne 2002-03, sauf indication contraire

\section{Par groupe de revenu (USD m)}

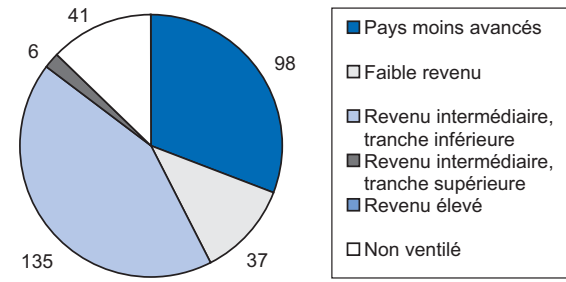

\section{Par région (USD $\mathrm{m}$ )}

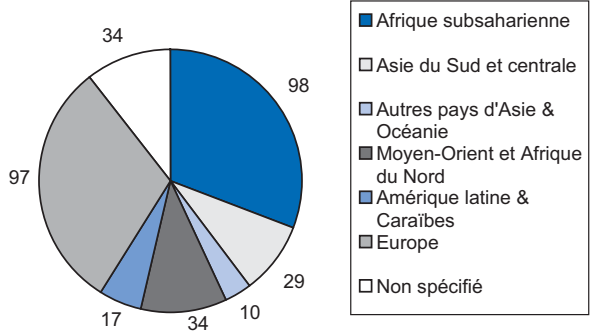




\section{Belgique}

Objectif :

Législation:

Déclaration de politique générale sur l'aide extérieure

Autres déclarations de politique :

Ministre responsable Autres ministres :

Ministère/organisme de développement principal :

Autres organismes/ ministères:

Structures de coordination interministérielles :

Principaux partenaires bilatéraux:

Principaux domaines de coopération :

Organismes ministériels consultatifs :
Développement durable par le biais de la lutte contre la pauvreté, sur la base du concept de partenariat et conformément aux critères de pertinence en matière de développement (Loi relative à la coopération internationale belge, 1999).

Loi relative à la coopération internationale belge (1999).

Policy Plan for Belgian International Co-operation.

Ministre de la Coopération au Développement.

Ministre des Affaires étrangères.

Direction générale de la coopération internationale (DGCl) du ministère fédéral des Affaires étrangères, Commerce extérieur et Coopération au Développement (www.dgdc.be).

Agence pour la coopération technique belge (CTB) (www.btcctb.org).

Ministère fédéral des Finances.

Ministère des Affaires étrangères.

Office national du Ducroire.

Gouvernement flamand

Gouvernement wallon.

La Belgique mène des programmes de coopération dans 18 pays principaux. En Afrique : Algérie, Bénin, Burundi, Congo, Mali, Maroc, Mozambique, Niger, Rwanda, Sénégal, Afrique du Sud, Tanzanie et Ouganda. En Amérique latine : Bolivie, Équateur et Pérou. En Asie : Viêt-nam et Zones sous administration palestinienne.

Santé, éducation, agriculture et sécurité alimentaire, infrastructure de base, prévention des conflits et consolidation de la société.

\section{BELGIQUE}

APD bilatérale brute, moyenne 2002-03, sauf indication contraire

\begin{tabular}{|lrrc|}
\hline & & & Variation \\
APD nette & $\mathbf{2 0 0 2}$ & $\mathbf{2 0 0 3}$ & $\mathbf{2 0 0 2 / 0 3}$ \\
Prix courants (USD m) & 1072 & 1853 & $73.0 \%$ \\
Prix constants (2002 USD m) & 1072 & 1508 & $40.7 \%$ \\
En euros (millions) & 1137 & 1640 & $44.3 \%$ \\
APD/RNB & $0.43 \%$ & $0.60 \%$ & \\
Aide bilatérale (\%) & $66 \%$ & $79 \%$ & \\
Aide publique nette (AP) & & & \\
Prix courants (USD m) & 97 & 163 & $67.6 \%$ \\
\hline
\end{tabular}

\begin{tabular}{|lr|}
\hline \multicolumn{2}{|c|}{ Dix principaux bénéficiaires } \\
d'APD/AP brute (USD m) \\
1 Congo, Rép. dém. & 415 \\
2 Tanzanie & 41 \\
3 Serbie \& Monténégro & 28 \\
4 Cameroun & 26 \\
5 Côte d'Ivoire & 25 \\
6 Rwanda & 21 \\
7 Burundi & 17 \\
8 Bolivie & 17 \\
9 Burkina Faso & 15 \\
10 Viêt-nam & 12 \\
\hline
\end{tabular}
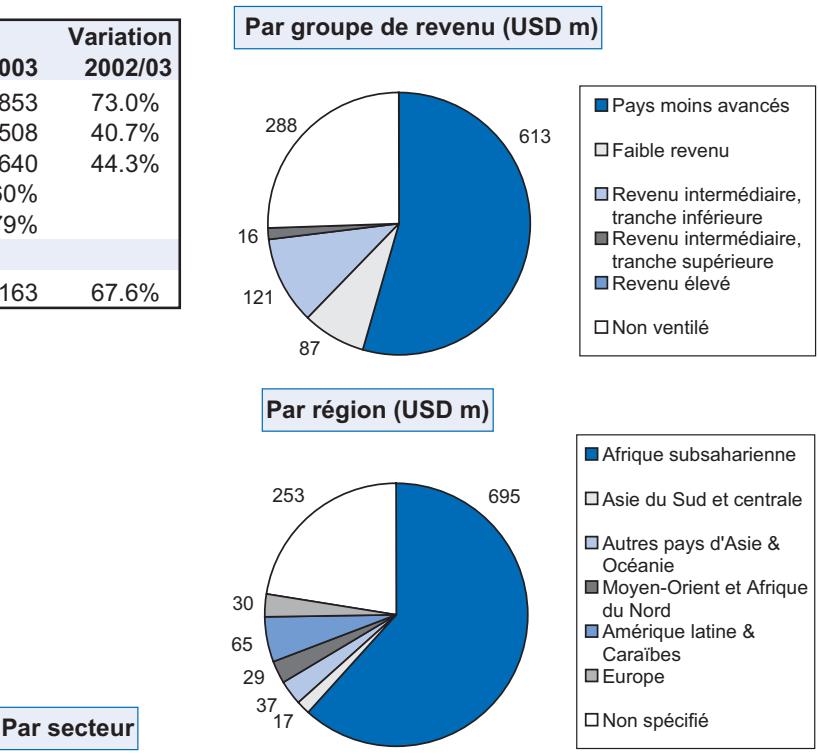

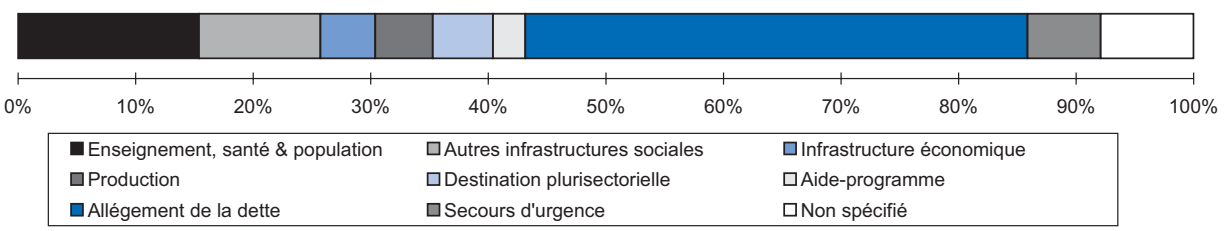




\section{Canada}

Objectif :

Favoriser le développement durable dans les pays en développement, afin de réduire la pauvreté et de contribuer à l'instauration d'un monde plus sûr, plus juste et plus prospère (Le Canada dans le monde, 1995).

Législation : Sans objet.

Déclaration de politique générale Le Canada dans le monde (1995).

sur l'aide extérieure :

Autres déclarations de politique : Le Canada contribue à un monde meilleur. Énoncé de politique en faveur d'une aide internationale plus efficace (2002).

Ministre responsable Ministre de la Coopération internationale.

Autres ministres: $\quad$ Ministre des Affaires étrangères.

Ministre des Finances.

Ministère/organisme de

développement principal :

Autres organismes/ministères : Ministère des Affaires étrangères (www.fac-aec.gc.ca).

Ministère du Commerce international (www.dfait-maeci.gc.ca).

Ministère des Finances (www.fin.gc.ca).

Centre de recherche pour le développement international (www.idrc.ca).

Droits et démocratie.

Santé Canada.

Travaux publics et services gouvernementaux Canada.

Environnement Canada.

Patrimoine canadien et Bureau du Conseil Privé.

Structures de coordination

interministérielles :

Principaux partenaires

bilatéraux:

Principaux domaines de coopération :

Organes ministériels

consultatifs :
L'assistance fournie par le Canada en matière de développement apporte un soutien aux activités bilatérales menées une centaine de pays, mais a sélectionné un nombre limité de pays en vue d'un partenariat renforcé.

Les domaines prioritaires pour l'ACDI concernent les besoins sociaux fondamentaux : éducation de base, santé, nutrition, VIH/sida ainsi que la protection de l'enfance, les droits de l'homme, la démocratie et la gouvernance.

\section{CANADA}

\begin{tabular}{|lrrr|}
\hline & & & Variation \\
APD nette & $\mathbf{2 0 0 2}$ & $\mathbf{2 0 0 3}$ & $\mathbf{2 0 0 2 / 0 3}$ \\
Prix courants (USD m) & 2004 & 2031 & $1.3 \%$ \\
Prix constants (2002 USD m) & 2004 & 1750 & $-12.7 \%$ \\
En dollars canadiens (millions) & 3147 & 2843 & $-9.6 \%$ \\
APD/RNB & $0.28 \%$ & $0.24 \%$ & \\
Aide bilatérale (\%) & $75 \%$ & $66 \%$ & \\
Aide publique nette (AP) & & & \\
Prix courants (USD m) & 104 & 102 & $-2.0 \%$ \\
\hline
\end{tabular}

\begin{tabular}{|lr|}
\hline \multicolumn{2}{|c|}{$\begin{array}{c}\text { Dix principaux bénéficiaires } \\
\text { d'APD/AP brute (USD } \mathrm{m} \text { ) }\end{array}$} \\
1 Pologne (AP) & 66 \\
2 États ex-Youg., non spécifié & 55 \\
3 Afghanistan & 54 \\
4 Cameroun & 50 \\
5 Côte d'Ivoire & 46 \\
6 Congo, Rép. dém. & 42 \\
7 Bangladesh & 35 \\
8 Chine & 33 \\
9 Inde & 31 \\
10 Irak & 24 \\
\hline
\end{tabular}

Par secteur

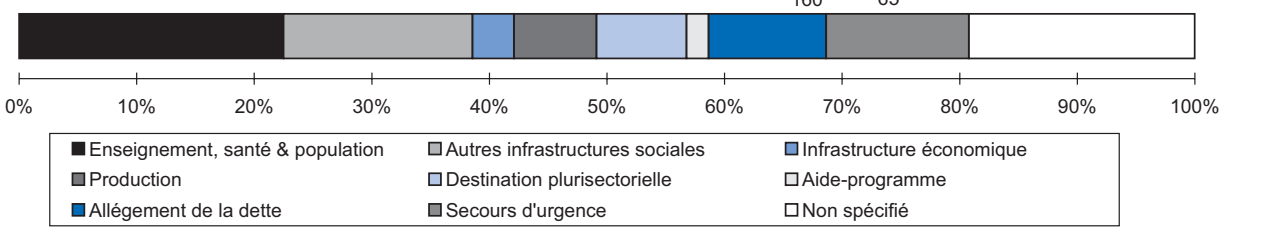




\section{Danemark}

Objectif :

Soutenir, par la coopération avec les gouvernements et les organismes publics des pays [en développement], les efforts que ceux-ci déploient pour favoriser la croissance économique, contribuant ainsi au progrès social et à l'indépendance politique, conformément aux objectifs et aux principes énoncés dans la Charte des Nations unies, et favoriser la compréhension mutuelle et la solidarité par la coopération culturelle (Loi de 1971 sur la coopération internationale pour le développement).

Législation: Loi de 1971 sur la coopération internationale pour le développement (modifiée en 1998 et en juin 2002).

Déclaration de politique Partnership 2000.

générale sur l'aide extérieure :

Autres déclarations de

A World of Difference: the Government's Vision for New Priorities in Danish Development Assistance 2004-2008.

politique :

Ministre responsable

Autres ministres:

Ministère/organisme de développement principal :

Ministre des Affaires étrangères.

Groupe Sud du ministère des Affaires étrangères (www.um.dk).

Autres organismes/

ministères :

Structures de coordination

interministérielles :

Principaux partenaires bilatéraux :

Les pays de programmes sont au nombre de 15. Pour l'Afrique : Bénin, Burkina Faso, Égypte, Ghana, Kenya, Mozambique, Tanzanie, Ouganda et Zambie. Pour l'Asie : Bangladesh, Bhoutan, Népal et Viêt-nam. Pour l'Amérique latine : Nicaragua et Bolivie.

Principaux domaines de coopération :

Secteurs sociaux - santé, éducation, eau et assainissement, infrastructure économique - ainsi que le transport, l'énergie et les secteurs de production - agriculture - et le secteur privé. Le principe consiste à intervenir au maximum dans trois ou quatre secteurs pour chaque pays de programme.

Organes ministériels consultatifs : Conseil de la coopération internationale pour le développement.

\section{DANEMARK}

APD bilatérale brute, moyenne 2002-03, sauf indication contraire

\begin{tabular}{|lrrc|}
\hline & & & Variation \\
APD nette & $\mathbf{2 0 0 2}$ & $\mathbf{2 0 0 3}$ & $\mathbf{2 0 0 2 / 0 3}$ \\
Prix courants (USD m) & 1643 & 1748 & $6.4 \%$ \\
Prix constants (2002 USD m) & 1643 & 1433 & $-12.8 \%$ \\
En couronnes dan. (millions) & 12956 & 11497 & $-11.3 \%$ \\
APD/RNB & $0.96 \%$ & $0.84 \%$ & \\
Aide bilatérale (\%) & $63 \%$ & $59 \%$ & \\
Aide publique nette (AP) & & & \\
Prix courants (USD m) & 167 & 202 & $20.4 \%$ \\
\hline
\end{tabular}

Par groupe de revenu (USD m)

\begin{tabular}{|lr|}
\hline \multicolumn{2}{|c|}{ Dix principaux bénéficiaires } \\
d'APD/AP brute (USD $\mathrm{m}$ ) \\
1 Tanzanie & 80 \\
2 Mozambique & 60 \\
3 Viêt-nam & 59 \\
4 Ghana & 54 \\
5 Ouganda & 49 \\
6 Bangladesh & 41 \\
7 Népal & 33 \\
8 Zambie & 31 \\
9 Bolivie & 30 \\
10 Égypte & 29 \\
\hline
\end{tabular}

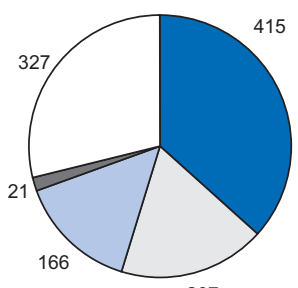

207
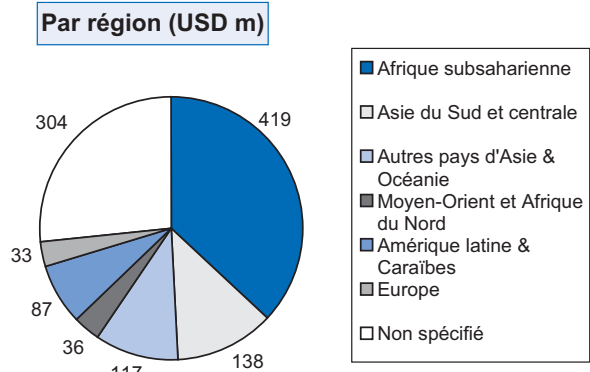

Par secteur

\begin{tabular}{|l|l|l|l|l|l|l|l|}
\hline & & & & & & \\
\hline
\end{tabular}




\section{Espagne}

Objectif :

Législation :

Déclaration de politique générale

sur l'aide extérieure :

Autres déclarations de politique :

Ministre responsable

Autres ministres :

Ministère/organisme de développement principal :

Autres organismes/ministères :

Favoriser le développement durable d'un point de vue humain, social et économique afin d'éliminer la pauvreté (Loi sur la coopération internationale pour le développement - Law on International Co-operation in Matters of Development, 1998).

Loi sur la coopération internationale pour le développement 1998.

: Plan directeur quadriennal (2001-2004).

Secrétaire d'État à la Coopération internationale et à l'Amérique latine, au ministère des Affaires étrangères.

Ministre de l'Économie.

Secrétaire d'État au Commerce et au Tourisme, au sein du ministère de l'Économie.

Secrétariat d'État pour la Coopération internationale et l'Amérique latine (SECIPI) et son organe exécutif, l'Agence espagnole de coopération internationale (AECl) (www.aeci.es).

Ministère de l'Économie (secrétariat d'État au Commerce et au Tourisme).

Ministère de l'Agriculture, de la Pêche et de l'Alimentation.

Ministère de la Défense.

Ministère de l'Éducation et de la Culture.

Ministère du Travail et des Affaires sociales.

Ministère de l'Environnement.

Ministère de la Santé et de la Consommation.

Ministère de l'Intérieur.

Ministère de la Justice.

Ministère des Administrations publiques.

Ministère des Travaux publics.

Ministère de la Science et de la Technologie.

Régions autonomes et municipalités.

Structures de coordination Comité interministériel pour la coopération internationale.

interministérielles: $\quad$ Commission interterritoriale sur la coopération pour le développement.

Principaux partenaires bilatéraux/ Vingt-neuf pays bénéficient du programme d'aide de l'Espagne. En Afrique : Algérie, Angola, Cap-Vert, Guinée équatoriale, pays bénéficiaires du programme Guinée-Bissau, Mauritanie, Maroc, Mozambique, Namibie, Sao Tomé et Principe, Sénégal, Afrique du Sud et Tunie. En Amérique latine : Bolivie, République dominicaine, Équateur, El Salvador, Guatemala, Honduras, Paraguay, Pérou et Nicaragua. En Asie : Chine, Philippines et Viêt-nam. En Europe : Albanie, Bosnie-Herzégovine et République fédérale de Yougoslavie.

Principaux domaines de coopération: Services sociaux de base, éducation, droits de l'homme, démocratie et développement de la société civile, environnement, culture, recherche scientifique et technologique.

Organes ministériels Conseil de coopération pour le développement consultatifs :

\section{ESPAGNE}

\begin{tabular}{|lrrc|}
\hline & & & Variation \\
APD nette & $\mathbf{2 0 0 2}$ & $\mathbf{2 0 0 3}$ & $\mathbf{2 0 0 2 / 0 3}$ \\
Prix courants (USD m) & 1712 & 1961 & $14.5 \%$ \\
Prix constants (2002 USD m) & 1712 & 1578 & $-7.8 \%$ \\
En euros (millions) & 1817 & 1736 & $-4.5 \%$ \\
APD/RNB & $0.26 \%$ & $0.23 \%$ & \\
Aide bilatérale (\%) & $58 \%$ & $59 \%$ & \\
Aide publique nette (AP) & & & \\
Prix courants (USD m) & 11 & 5 & $-53.7 \%$ \\
\hline
\end{tabular}

\begin{tabular}{|lr|}
\hline \multicolumn{2}{|c|}{$\begin{array}{c}\text { Dix principaux bénéficiaires } \\
\text { d'APD/AP brute (USD } \mathrm{m} \text { ) }\end{array}$} \\
1 Bolivie & 69 \\
2 Chine & 55 \\
3 Nicaragua & 50 \\
4 Honduras & 47 \\
5 Pérou & 42 \\
6 El Salvador & 41 \\
7 Équateur & 41 \\
8 Maroc & 37 \\
9 États ex-Youg., non spécifié & 33 \\
10 Dominicaine, Rép. & 32 \\
\hline
\end{tabular}

APD bilatérale brute, moyenne 2002-03, sauf indication contraire

\section{Par groupe de revenu (USD $\mathrm{m}$ )}

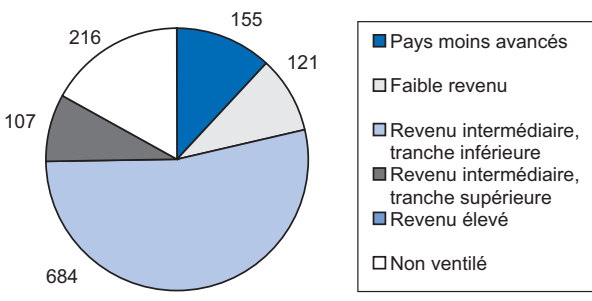

Par région (USD $\mathrm{m}$ )

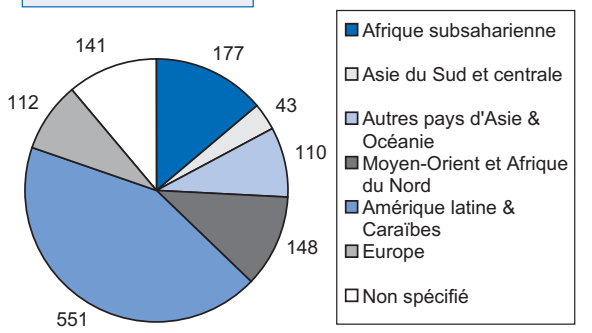

\begin{tabular}{|l|l|l|l|l|l|l|l|}
\hline & & & & & \\
\hline
\end{tabular}




\section{États-Unis}

Objectif :

Législation:

Déclaration de politique générale sur l'aide extérieure :

Autres déclarations de politique:

Ministre responsable Autres ministres:

Ministère/organisme de développement principal :

Autres organismes/ministères :

Structures de coordination interministérielles :

Principaux partenaires bilatéraux/pays prioritaires :

Principaux domaines de coopération :

Organes ministériels consultatifs :
Loi sur l'aide étrangère (Foreign Assistance Act) de 1961, modifiée.

US Department of State and US Agency for International Development, Strategic Plan: Fiscal Years 2004-2009.

White Paper: US Foreign Aid: Meeting the Challenges of the Twenty-First Century, January 2004

National Security Strategy (2002).

Foreign Aid in the National Interest: Promoting Freedom, Security and Opportunity (2002).

Administrateur de l'Agence USAID (qui rend compte au Ministre des Affaires étrangères).

Ministre des Affaires étrangères.

Ministre des Finances.

Agence des États-Unis pour le développement international (USAID) (www.usaid.gov).

Ministère des Affaires étrangères (State Department) (www.state.gov)

Secrétariat aux Affaires internationales (Office of International Affairs) du ministère des Finances (Treasury) - (www.ustreas.gov).

The Millennium Challenge Corporation (www.mcc.gov).

Ministères de l'Agriculture, de la Défense, de la Santé et des Affaires sociales, de l'Intérieur, etc.

Peace Corps (www. peacecorps.gov/home.htm).

Le National Security Council est chargé de la coordination générale entre les organismes gouvernementaux.

Les Comités de coordination des politiques (Policy Co-ordination Committees - PCC) ont été mis en place, de façon ponctuelle ou permanente, pour assurer la coordination sur des questions d'orientation bien précises. Le PCC chargé du développement est présidé par le ministère des Affaires étrangères.

L'USAID a des bureaux dans plus de 70 pays, dont 22 en Afrique, 20 au Proche-Orient et en Asie, et 17 en Amérique latine.

Agriculture, gestion des conflits, démocratie et gouvernance, croissance économique et commerce, éducation, environnement et population, santé et nutrition.

Advisory Committee on Voluntary Foreign Aid (ACVFA).

\section{ÉTATS-UNIS}

APD bilatérale brute, moyenne 2002-03, sauf indication contraire

\begin{tabular}{|lrrc|}
\hline & & & Variation \\
APD nette & $\mathbf{2 0 0 2}$ & $\mathbf{2 0 0 3}$ & $\mathbf{2 0 0 2 / 0 3}$ \\
Prix courants (USD m) & 13290 & 16254 & $22.3 \%$ \\
Prix constants (2002 USD m) & 13290 & 15997 & $20.4 \%$ \\
& & & \\
APD/RNB & $0.13 \%$ & $0.15 \%$ & \\
Aide bilatérale (\%) & $80 \%$ & $90 \%$ & \\
Aide publique nette (AP) & & & \\
Prix courants (USD m) & 2313 & 1471 & $-36.4 \%$ \\
\hline
\end{tabular}

\begin{tabular}{|cr|}
\hline \multicolumn{2}{|c|}{$\begin{array}{c}\text { Dix principaux bénéficiaires } \\
\text { d'APD/AP brute (USD m) }\end{array}$} \\
1 Égypte & 831 \\
2 Russie (AP) & 808 \\
3 Irak & 775 \\
4 Congo, Rép. dém. & 749 \\
5 Israël (AP) & 666 \\
6 Pakistan & 656 \\
7 Jordanie & 622 \\
8 Colombie & 513 \\
9 Afghanistan & 427 \\
10 Éthiopie & 374 \\
\hline
\end{tabular}

Par groupe de revenu (USD $\mathrm{m}$ )

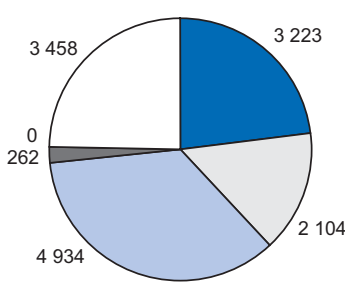

口Pays moins avancés

口Faible revenu

$\square$ Revenu intermédiaire,

tranche inférieure

口Revenu intermédiaire,

tranche supérieure

$\square$ Revenu élevé

$\square$ Non ventilé
Par région (USD $\mathrm{m}$ )

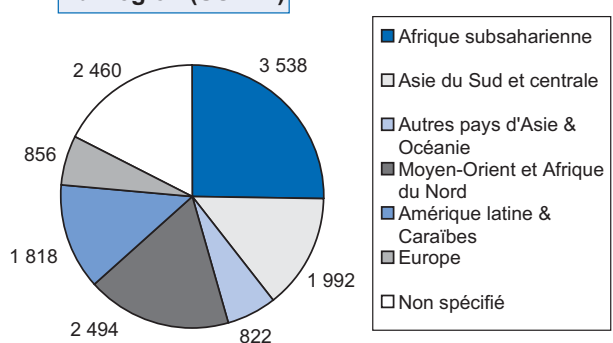

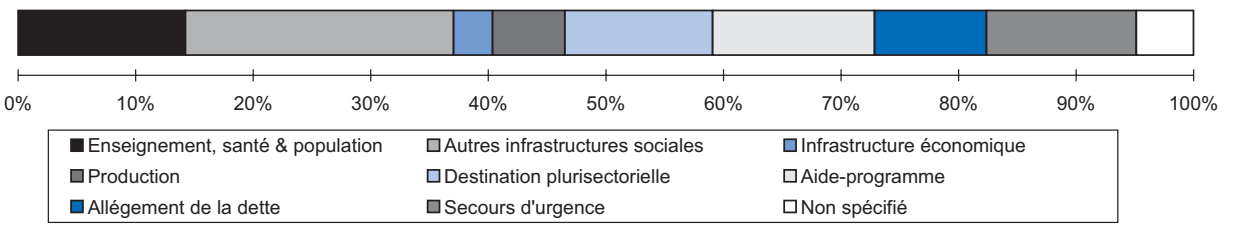


Finlande

Objectif :

Législation:

Déclaration de politique générale sur l'aide extérieure :

Autres déclarations

de politique :

Ministre responsable

Autres ministres:

Ministère/organisme

de développement principal :

Autres organismes/ministères :

Structures de coordination

interministérielles :

\section{Principaux partenaires}

bilatéraux :

Principaux domaines de coopération :

Organes ministériels

consultatifs :
Contribuer à l'éradication de la grande pauvreté (White Paper on Development Policy 2004).

Sans objet.

Development Policy, Government Resolution (2004).

Government Decisions-in-Principle (2001)

Ministre du Commerce et du développement.

Ministre des Affaires étrangères.

Ministre des Affaires étrangères

( http://formin.finland.fi/english)

Finnfund ( www. finnfund.fi)

The Service Centre for Development Cooperation (KEPA) (www.kepa.fi).

Les pays partenaires à long terme sont au nombre de huit. En Afrique : Éthiopie, Kenya, Mozambique, Tanzanie et Zambie. En Asie : Népal et Viêt-nam. En Amérique latine : Nicaragua.

La Finlande soutient les efforts des pays partenaires pour atteindre les OMD. Les secteurs devant bénéficier d'un soutien sont sélectionnés après analyse, au cas par cas, de la valeur ajoutée de l'aide finlandaise.

The Development Policy Committee.

\section{FINLANDE}

\begin{tabular}{|lrrc|}
\hline & & & Variation \\
APD nette & $\mathbf{2 0 0 2}$ & $\mathbf{2 0 0 3}$ & $\mathbf{2 0 0 2 / 0 3}$ \\
Prix courants (USD m) & 462 & 558 & $20.8 \%$ \\
Prix constants (2002 USD m) & 462 & 464 & $0.3 \%$ \\
En euros (millions) & 490 & 494 & $0.8 \%$ \\
APD/RNB & $0.35 \%$ & $0.35 \%$ & \\
Aide bilatérale (\%) & $54 \%$ & $55 \%$ & \\
Aide publique nette (AP) & & & \\
Prix courants (USD m) & 67 & 82 & $22.4 \%$ \\
\hline
\end{tabular}

\begin{tabular}{|lr|}
\hline \multicolumn{2}{|c|}{ Dix principaux bénéficiaires } \\
d'APD/AP brute (USD m) \\
1 Mozambique & 17 \\
2 Afghanistan & 14 \\
3 Russie (AP) & 13 \\
4 Tanzanie & 13 \\
5 Serbie \& Monténégro & 8 \\
6 Namibie & 8 \\
7 Afrique du Sud & 8 \\
8 Viêt-nam & 8 \\
9 Nicaragua & 7 \\
10 Éthiopie & 7 \\
\hline
\end{tabular}

APD bilatérale brute, moyenne 2002-03, sauf indication contraire
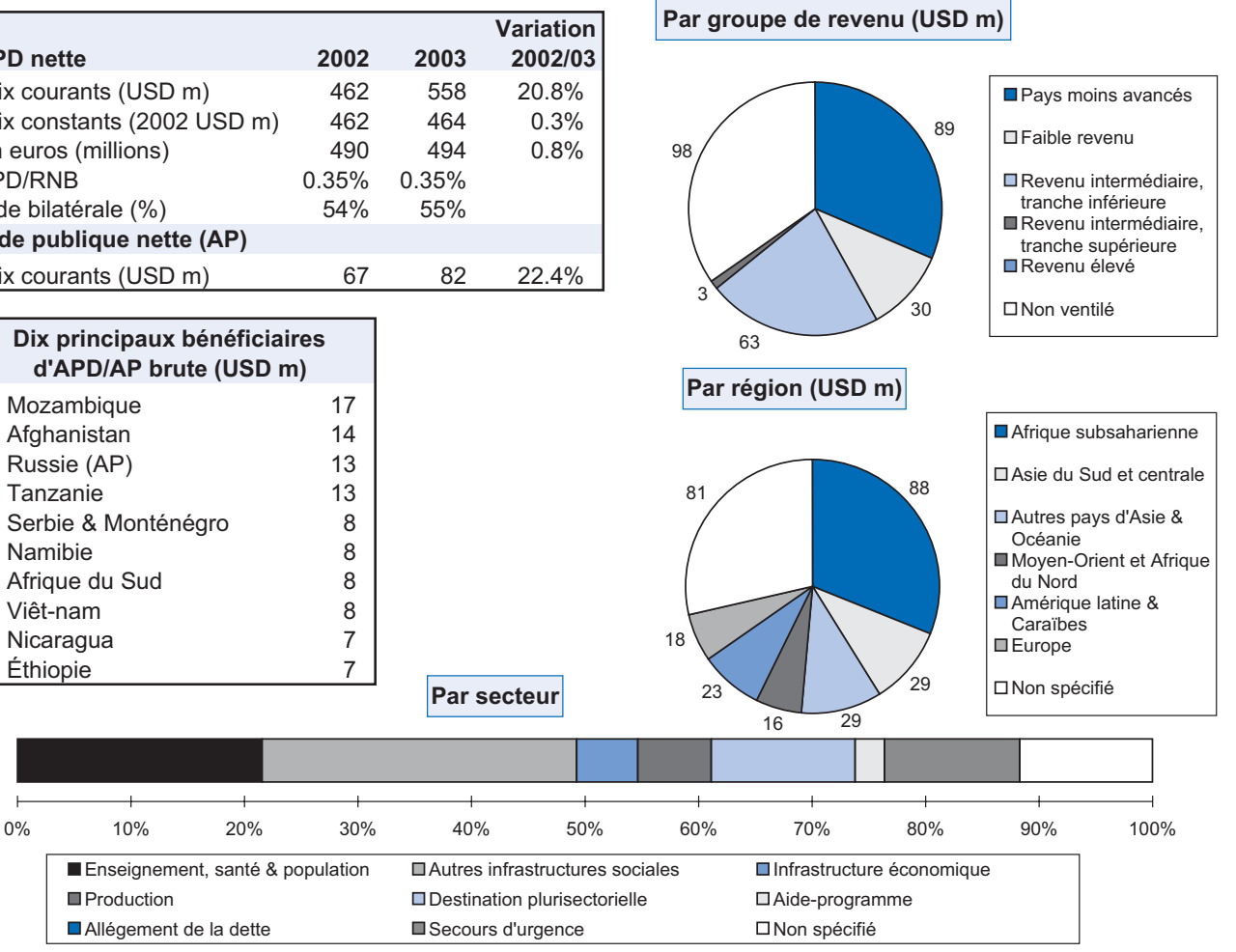


\section{France}

Objectif :

Législation :

Déclaration de politique générale sur l'aide extérieure

Autres déclarations de

politique :

Ministres :

Autres ministres :

Ministère/organisme de développement principal :

Autres organismes/ ministères :

Structures de coordination interministérielles :

Principaux partenaires bilatéraux:

Principaux domaines de coopération :

Organes ministériels consultatifs :
Sans objet.

Ministre de l'Économie, des Finances et de l'Industrie.

Direction générale de la coopération internationale et du développement (DGCID), au sein du ministère des Affaires étrangères (www.france.diplomatie.fr).

Ministère de l'Économie, des Finances et de l'Industrie (www.mineti.gouv.fr/minefi/europe/index.htm).

Agence française de développement (AFD) (www.afd.fr).

Ministère de l'Éducation nationale, de l'Enseignement supérieur et de la Recherche.

Ministère de l'Agriculture.

Ministère de l'Intérieur.

Ministère de l'Environnement.

Ministère de la Culture et de la Communication.

Ministère des Affaires sociales.

Ministère de l'Équipement.

Comité interministériel de la coopération internationale et du développement (CICID).

La Zone de solidarité prioritaire (ZSP) comprend 54 pays.

Approvisionnement en eau et assainissement, éducation, santé et VIH/sida, agriculture et développement rural, infrastructure. Haut Conseil de la coopération internationale (HCCl).

\section{FRANCE}

\begin{tabular}{|lrrc|}
\hline & & & Variation \\
APD nette & $\mathbf{2 0 0 2}$ & $\mathbf{2 0 0 3}$ & $\mathbf{2 0 0 2 / 0 3}$ \\
Prix courants (USD m) & 5486 & 7253 & $32.2 \%$ \\
Prix constants (2002 USD m) & 5486 & 5961 & $8.7 \%$ \\
En euros (millions) & 5821 & 6420 & $10.3 \%$ \\
APD/RNB & $0.38 \%$ & $0.41 \%$ & \\
Aide bilatérale (\%) & $66 \%$ & $72 \%$ & \\
Aide publique nette (AP) & & & \\
Prix courants (USD m) & 1464 & 2027 & $38.4 \%$ \\
\hline
\end{tabular}

\begin{tabular}{|lr|}
\hline \multicolumn{2}{|c|}{ Dix principaux bénéficiaires } \\
d'APD/AP brute (USD m) \\
1 Congo, Rép. dém. & 704 \\
2 Polynésie francaise (AP) & 490 \\
3 Côte d'Ivoire & 447 \\
4 Nouvelle-Calédonie (AP) & 421 \\
5 Cameroun & 302 \\
6 Pakistan & 250 \\
7 Maroc & 245 \\
8 Mozambique & 240 \\
9 Pologne (AP) & 185 \\
10 Serbie \& Monténégro & 156 \\
\hline
\end{tabular}

Par secteur
APD bilatérale brute, moyenne 2002-03, sauf indication contraire

\section{Par groupe de revenu (USD m)}

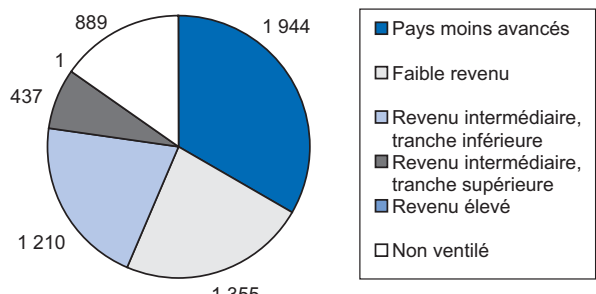

Par région (USD $\mathrm{m}$ )

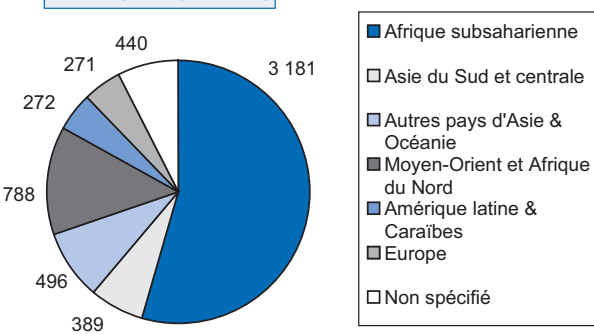

\begin{tabular}{|l|l|l|l|l|ll|l|}
\hline & & & & & & & \\
\hline
\end{tabular}




\section{Grèce}

Objectif :

Contribuer au développement économique et social, à la lutte contre la pauvreté, au renforcement de la démocratie et de l'État de droit, au respect des droits de l'homme et des libertés fondamentales, à l'égalité des sexes et à la protection de l'environnement. (Deuxième programme quinquennal de coopération pour le développement 2002-2006 - Second Medium-Term Five Year Development Co-operation Programme 2002-2006)

Législation :

Loi 2731/1999 (Journal officiel 138/A/5-7-1999) et décret présidentiel 224/2000 (Journal officiel 193/A/6-9-2000).

Déclaration de politique générale sur l'aide extérieure :

Autres déclarations de politique :

Ministres :

Deuxième programme quinquennal de coopération pour le développement (2002-2006).

Autres ministres

Ministère/organisme de développement principal :

Autres organismes/ministères:

Ministre délégué auprès du ministre des Affaires étrangères, chargé des Relations économiques internationales et de la Coopération pour le Développement.

Ministre de l'Économie et des Finances.

Coopération internationale pour le développement (YDAS ou HELLENIC AID), ministère des Affaires étrangères (www.mfa.gr).

Ministère de l'Économie et des Finances.

Ministère de la Défense nationale.

Ministère de l'Agriculture.

Ministère de l'Environnement.

Ministère de l'Intérieur, de la Fonction publique et de la Décentralisation.

Ministère de l'Environnement, de l'Aménagement du territoire et des Travaux publics.

Ministère de l'Éducation nationale et des Cultes.

Ministère de la Santé et de la Solidarité sociale.

Ministère de la Marine marchande.

Commission grecque pour le commerce extérieur (Hellenic Foreign Trade Board).

Association grecque des petites et moyennes entreprises et des artisans.

Office national du tourisme.

Organisme national pour l'emploi.

Structures de coordination interministérielles:

Comité d'organisation et de coordination des relations économiques internationales (EOSDOS)

Principaux partenaires bilatéraux: Les pays partenaires sont au nombre de 18. Dans les Balkans : Albanie, Bosnie-Herzégovine, Bulgarie, République fédérale de Yougoslavie, ex-République yougoslave de Macédoine et Roumanie. Dans la région de la mer Morte : Arménie et Géorgie. Au Proche-Orient : Afghanistan, Irak, Jordanie, Liban, Zones sous administration palestinienne, Syrie et Turquie. En Afrique subsaharienne : Éthiopie, Érythrée et Côte d'Ivoire.

Principaux domaines de coopération:

Infrastructure et formation professionnelle dans le domaine de l'enseignement fondamental et secondaire, infrastructure dans le domaine de la santé, approvisionnement en eau et assainissement, environnement et agriculture, soutien aux activités en faveur de la démocratisation et du respect des droits de l'homme, au renforcement des institutions, au micro-crédit et à la production de revenus.

Organes ministériels consultatifs

GRÈCE

\begin{tabular}{|lrrc|}
\hline & & & Variation \\
APD nette & $\mathbf{2 0 0 2}$ & $\mathbf{2 0 0 3}$ & $\mathbf{2 0 0 2 / 0 3}$ \\
Prix courants (USD m) & 276 & 362 & $31.2 \%$ \\
Prix constants (2002 USD m) & 276 & 292 & $5.7 \%$ \\
En euros (millions) & 293 & 321 & $9.4 \%$ \\
APD/RNB & $0.21 \%$ & $0.21 \%$ & \\
Aide bilatérale (\%) & $39 \%$ & $63 \%$ & \\
Aide publique nette (AP) & & & \\
Prix courants (USD m) & 16 & 81 & $410.9 \%$ \\
\hline
\end{tabular}

\section{Dix principaux bénéficiaires d'APD/AP brute (USD m)}

1 Albanie

2 Serbie \& Monténégro

3 ERY Macédoine

4 Afghanistan

5 Bosnie-Herzégovine

6 Bulgarie (AP)

7 Géorgie

8 Turquie

9 Irak

10 Ukraine (AP)

3

APD bilatérale brute, moyenne 2002-03, sauf indication contraire

Par groupe de revenu (USD m)

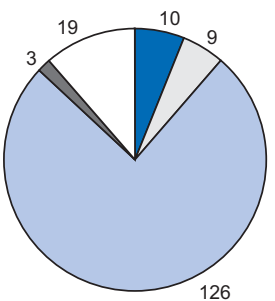

口Pays moins avancés

$\square$ Faible revenu

$\square$ Revenu intermédiaire tranche inférieure $\square$ Revenu intermédiaire tranche supérieure $\square$ Revenu élev

$\square$ Non ventile
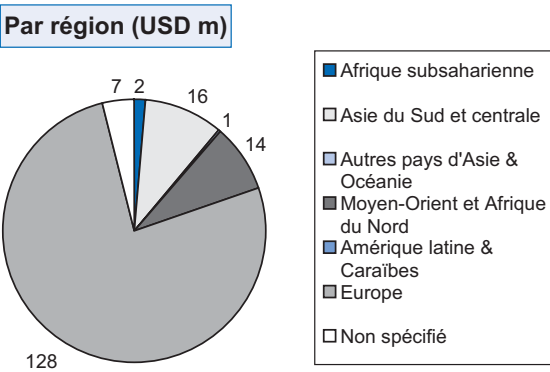

128

\begin{tabular}{|l|lllll|l|l|l|}
\hline & & & & & \\
\hline
\end{tabular}




\section{Irlande}

Objectif :

Lutte contre la pauvreté et promotion du développement durable dans certains des pays les plus pauvres de la planète (Livre blanc de politique étrangère - White Paper on Foreign Policy 1996).

Législation : Sans objet.

Déclaration de politique Livre blanc de politique étrangère (1996).

générale sur l'aide extérieure :

Autres déclarations de Rapport de la Commission d'examen de l'aide irlandaise (2002).

politique :

Ministre responsable

Autres ministres :

Ministère/organisme de développement principal :

Autres organismes/

ministères :

Structures de coordination interministérielles :

Principaux partenaires bilatéraux/pays de programme : oriental. Le DCl est également très actif en ce qui concerne l'Afghanistan, les Zones sous administration palestinienne et l'Afrique du Sud.

Principaux domaines de coopération :

Éducation, santé, approvisionnement en eau et assainissement, gouvernance.

Organes ministériels consultatifs :

\section{IRLANDE}

\begin{tabular}{|lrrc|}
\hline & & & Variation \\
APD nette & $\mathbf{2 0 0 2}$ & $\mathbf{2 0 0 3}$ & $\mathbf{2 0 0 2 / 0 3}$ \\
Prix courants (USD m) & 398 & 504 & $26.6 \%$ \\
Prix constants (2002 USD m) & 398 & 413 & $3.8 \%$ \\
En euros (millions) & 422 & 446 & $5.6 \%$ \\
APD/RNB & $0.40 \%$ & $0.39 \%$ & \\
Aide bilatérale (\%) & $67 \%$ & $70 \%$ & \\
Aide publique nette (AP) & & & \\
Prix courants (USD m) & 26 & 1 & $-95.8 \%$ \\
\hline
\end{tabular}

\begin{tabular}{|lr|}
\hline \multicolumn{2}{|c|}{ Dix principaux bénéficiaires } \\
\multicolumn{2}{|c|}{ d'APD/AP brute (USD m) } \\
1 Ouganda & 41 \\
2 Mozambique & 35 \\
3 Éthiopie & 29 \\
4 Tanzanie & 26 \\
5 Zambie & 21 \\
6 Afrique du Sud & 15 \\
7 Lesotho & 12 \\
8 Afghanistan & 6 \\
9 Zones/adm.palestinienne & 5 \\
10 Kenya & 5 \\
\hline
\end{tabular}

APD bilatérale brute, moyenne 2002-03, sauf indication contraire Par groupe de revenu (USD m)

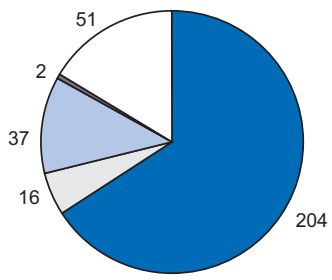

$\square$ Pays moins avancés $\square$ Faible revenu $\square$ Revenu intermédiaire, tranche inférieure 口Revenu intermédiaire, tranche supérieure $\square$ Revenu élevé

$\square$ Non ventilé
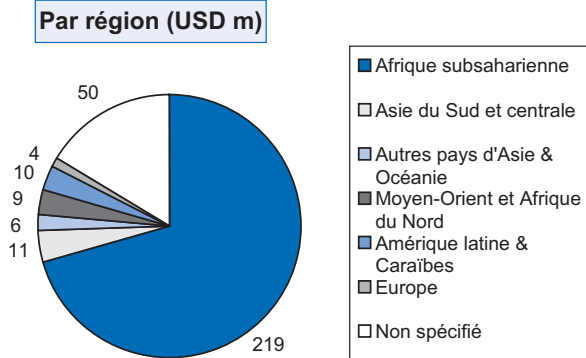

\begin{tabular}{|l|lll|l|l|l|l|l|}
\hline & & & & & & \\
\hline
\end{tabular}




\section{Italie}

Objectif :

La coopération pour le développement fait partie intégrante de la politique étrangère de l'Italie et repose sur les idéaux de solidarité entre les peuples et de respect des droits humains fondamentaux, conformément aux principes sanctionnés par les Nations unies et dans les conventions entre la Commission européenne (CE) et les États d'Afrique, des Caraïbes et du Pacifique (ACP) (Loi $n^{0}$ 49/87, Annexe 1).

Législation : Loi $n^{\circ}$ 49/87 (1987).

Déclaration de politique générale sur l'aide extérieure :

Autres déclarations de politique :

Ministre responsable

Orientations du Comité interministériel de planification économique (CIPE) (1995).

Ministre des Affaires étrangères, assisté de quatre sous-secrétaires d’État.

Ministère/organisme de

développement principal :

Autres organismes/ministères :

Ministre de l'Économie et des Finances.

Direction générale de la coopération pour le développement (DGCS) du ministère des Affaires étrangères (www.esteri.it).

Ministère de l'Économie et des Finances.

Ministère des Activités productives.

Ministère de l'Environnement et de la Protection du territoire.

Ministère de l'Intérieur.

Ministère de l'Éducation, de l'Université et de la Recherche.

Ministère de la Justice.

Présidence du Conseil des ministres.

Ministère de l'Agriculture.

Régions et municipalités.

Structures de coordination Comité interministériel de planification économique (CIPE).

interministérielles :

Principaux partenaires bilatéraux : L'aide italienne est concentrée sur 16 pays. En Afrique du Nord : Algérie, Égypte, Maroc et Tunisie. En Afrique subsaharienne : Angola, Érythrée, Éthiopie, Mozambique, Afrique du Sud et Ouganda. En Asie : Chine et Inde. Au Proche-Orient : Jordanie, Liban, Zones sous administration palestinienne et Syrie.

Principaux domaines de Santé, éducation, développement rural et sécurité alimentaire, assistance humanitaire et développement du secteur privé coopération : (en particulier PME)

Organes ministériels consultatifs

\section{ITALIE}

\begin{tabular}{|lrrr|}
\hline APD nette & $\mathbf{2 0 0 2}$ & $\mathbf{2 0 0 3}$ & Variation \\
Prix courants (USD m) & 2332 & 2433 & $4.3 \%$ \\
Prix constants (2002 USD m) & 2332 & 1976 & $-15.3 \%$ \\
En euros (millions) & 2475 & 2153 & $-13.0 \%$ \\
APD/RNB & $0.20 \%$ & $0.17 \%$ & \\
Aide bilatérale (\%) & $43 \%$ & $44 \%$ & \\
Aide publique nette (AP) & & & \\
Prix courants (USD m) & - & 497 & - \\
\hline
\end{tabular}

\section{Dix principaux bénéficiaires} d'APD/AP brute (USD $\mathrm{m}$ )

1 Mozambique

2 Congo, Rép. dém

3 Tanzanie

4 Éthiopie

5 Tunisie

6 Guinée-Bissau

7 Afghanistan

8 Chine

9 Zones/adm.palestinienne

10 Albanie

\begin{tabular}{|c|c|c|c|c|c|c|c|c|c|c|}
\hline \multirow[t]{2}{*}{$0 \%$} & $10 \%$ & $20 \%$ & $30 \%$ & $40 \%$ & $50 \%$ & $60 \%$ & $70 \%$ & $80 \%$ & $90 \%$ & \multirow[t]{2}{*}{$100 \%$} \\
\hline & \multicolumn{3}{|c|}{$\begin{array}{l}\text { Enseignement, santé \& population } \\
\text { 口Production } \\
\text { 口Allégement de la dette }\end{array}$} & \multicolumn{3}{|c|}{$\begin{array}{l}\square \text { Autres infrastructures sociales } \\
\square \text { Destination plurisectorielle } \\
\square \text { Secours d'urgence }\end{array}$} & \multicolumn{3}{|c|}{$\begin{array}{l}\square \text { Infrastructure économique } \\
\square \text { Aide-programme } \\
\square \text { Non spécifié }\end{array}$} & \\
\hline
\end{tabular}

APD bilatérale brute, moyenne 2002-03, sauf indication contraire

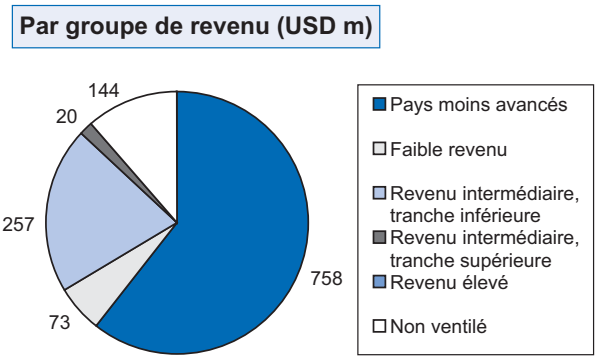

Par région (USD $\mathrm{m}$ )

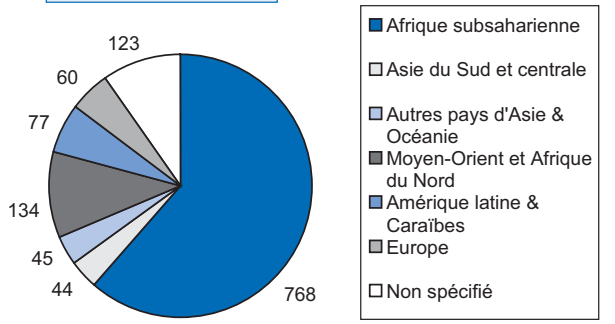




\section{Japon}

Objectif :

Législation :

Déclaration de politique générale sur l'aide extérieure :

Autres déclarations de politique :

Ministre responsable :

Autres ministres :

Ministère/organisme de développement principal :

Autres organismes/ministères :

Structures de coordination interministérielles :

Principaux partenaires bilatéraux/ pays de programme :

Principaux domaines de coopération :

Organes ministériels consultatifs
Contribuer à la paix et au développement de la communauté internationale et, partant, à la sécurité et à la prospérité du Japon (Charte APD 2003).

Charte japonaise pour l'aide publique au développement (2003).

Charte japonaise pour l'aide publique au développement (1992, mise à jour en 2003).

Livre blanc sur l'aide publique au développement (2003).

Ministre des Affaires étrangères.

Ministre des Finances.

Bureau de la coopération économique au ministère des Affaires étrangères (www.mofa.go.jp/policy/oda).

Agence japonaise de coopération internationale (JICA) (www.jica.go.jp).

Banque japonaise de coopération internationale (JBIC) (www.jbic.go.jp)

Ministère des Finances (www.mof.go.jp).

Ministère de l'Économie, du Commerce et de l'Industrie (www.meti.go.jp).

Ministère de l'Éducation, de la Culture, des Sports, de la Science et de la Technologie.

Ministère de l'Agriculture, de la Forêt et de la Pêche.

Ministère de la Santé, du Travail et de la Protection sociale.

Ministère de l'Aménagement du territoire, de l'Infrastructure et des Transports.

Ministère des Affaires intérieures et de la Communication.

Ministère de la Justice.

Ministère de l'Environnement.

Cabinet Office.

Agence des services financiers.

Agence de police nationale.

Conseil de coopération économique étrangère.

Conférence interministérielle sur l'APD.

Le Japon est actif dans plus de 140 pays, les programmes de coopération les plus importants étant concentrés en Asie.

Secteurs prioritaires énoncés dans la charte APD. Réduction de la pauvreté (éducation, santé, approvisionnement en eau et assainissement, agriculture). Croissance durable (infrastructure, commerce et investissement).

Problèmes de portée mondiale (environnement, traitement des maladies infectieuses, population, alimentation, énergie, catastrophes naturelles, lutte contre le trafic de stupéfiants et le crime organisé).

Construction de la paix (prévention des conflits et aide d'urgence).

Commission sur la stratégie globale en matière d'APD.

\section{JAPON}

\begin{tabular}{|lrrc|}
\hline & & & Variation \\
APD nette & $\mathbf{2 0 0 2}$ & $\mathbf{2 0 0 3}$ & $\mathbf{2 0 0 2 / 0 3}$ \\
Prix courants (USD m) & 9283 & 8880 & $-4.3 \%$ \\
Prix constants (2002 USD m) & 9283 & 8429 & $-9.2 \%$ \\
En yen (milliards) & 1162 & 1029 & $-11.5 \%$ \\
APD/RNB & $0.23 \%$ & $0.20 \%$ & \\
Aide bilatérale (\%) & $72 \%$ & $71 \%$ & \\
Aide publique nette (AP) & & & \\
Prix courants (USD m) & 99 & -219 & $-322.1 \%$ \\
\hline
\end{tabular}

\begin{tabular}{|lr|}
\hline \multicolumn{2}{|c|}{$\begin{array}{c}\text { Dix principaux bénéficiaires } \\
\text { d'APD/AP brute (USD } \mathbf{~ m} \text { ) }\end{array}$} \\
1 Chine & 1297 \\
2 Indonésie & 891 \\
3 Philippines & 810 \\
4 Inde & 768 \\
5 Thaïlande & 651 \\
6 Viêt-nam & 452 \\
7 Pakistan & 284 \\
8 Bangladesh & 262 \\
9 Sri Lanka & 249 \\
10 Malaisie & 187 \\
\hline
\end{tabular}

10 Malaisie

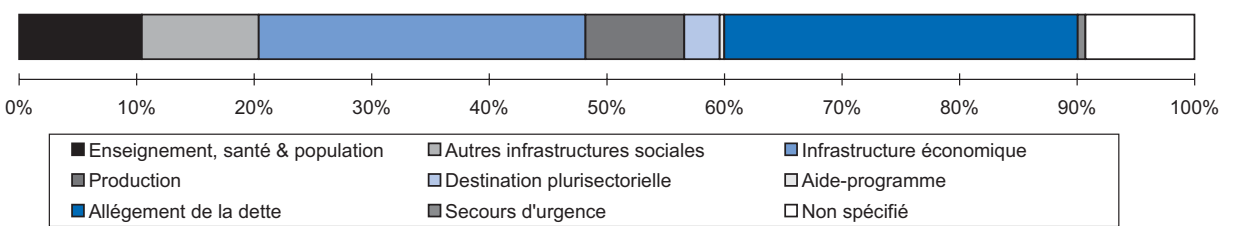

APD bilatérale brute, moyenne 2002-03, sauf indication contraire Par groupe de revenu (USD m)

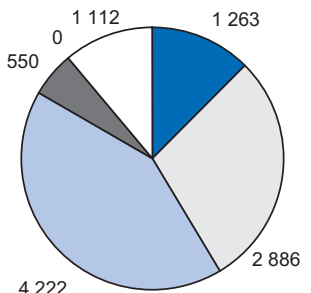

口Pays moins avancés

$\square$ Faible revenu

$\square$ Revenu intermédiaire, tranche inférieure

$\square$ Revenu intermédiaire,

tranche supérieure

$\square$ Revenu élevé

$\square$ Non ventilé

\section{Par région (USD $\mathrm{m}$ )}

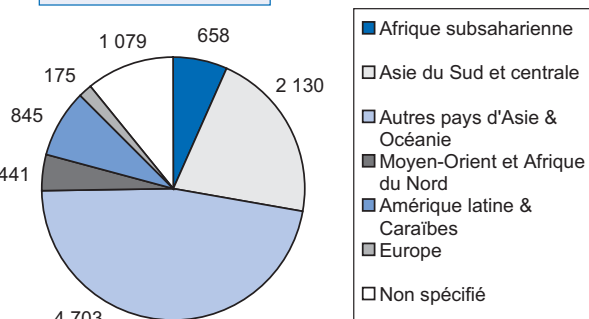




\section{Luxembourg}

Objectif :

Développement durable et lutte contre la pauvreté (Une politique de solidarité avec les plus démunis - A Policy of Solidarity With Those Most in Need (1999).

Législation :

Loi sur la coopération pour le développement (1996).

Déclaration de politique générale sur l'aide extérieure :

Autres déclarations de Une politique de solidarité avec les plus démunis - A Policy of Solidarity With Those Most in Need (1999).

politique :

Ministre responsable : $\quad$ Ministre de la Coopération et de l'Action humanitaire.

Autres ministres:

Ministre des Affaires étrangères et du Commerce extérieur.

Ministre des Finances.

Ministère/organisme de développement principal :

Direction de la coopération au développement au ministère des Affaires étrangères (www.mae.lu).

Autres organismes/ministères: Ministère des Finances.

Structures de coordination Comité interministériel pour la coopération au développement.

interministérielles :

Principaux partenaires

bilatéraux :

Les pays « cibles » sont au nombre de dix. En Afrique : Burkina Faso, Cap-Vert, Mali, Namibie, Niger et Sénégal. En Asie : Laos et Viêt-nam. En Amérique latine : El Salvador et Nicaragua. En outre, 20 pays donnent lieu à des projets. En Afrique : Burundi, Guinée, Maroc, île Maurice, Rwanda, Sao Tomé et Principe, Afrique du Sud et Tunisie. En Asie : Chine, Timor oriental, Inde et Mongolie. En Amérique latine : Brésil, Chili, Équateur et Pérou. En Europe : Albanie, Bosnie-Herzégovine, Croatie et République fédérale de Yougoslavie.

Principaux domaines de Santé, approvisionnement en eau et assainissement, éducation, autres services sociaux et développement rural coopération : intégré.

Organes ministériels

consultatifs :

\section{LUXEMBOURG}

\begin{tabular}{|lrrc|}
\hline & & & Variation \\
APD nette & $\mathbf{2 0 0 2}$ & $\mathbf{2 0 0 3}$ & $\mathbf{2 0 0 2 / 0 3}$ \\
Prix courants (USD m) & 147 & 194 & $32.1 \%$ \\
Prix constants (2002 USD m) & 147 & 159 & $8.4 \%$ \\
En euros (millions) & 156 & 172 & $10.2 \%$ \\
APD/RNB & $0.77 \%$ & $0.81 \%$ & \\
Aide bilatérale (\%) & $79 \%$ & $77 \%$ & \\
Aide publique nette (AP) & & & \\
Prix courants (USD m) & 10 & 6 & $-38.7 \%$ \\
\hline
\end{tabular}

\begin{tabular}{|lr|}
\hline \multicolumn{2}{|c|}{ Dix principaux bénéficiaires } \\
d'APD/AP brute (USD m) \\
1 Cap-Vert & 9 \\
2 Viêt-nam & 8 \\
3 Burkina Faso & 6 \\
4 Laos & 6 \\
5 Mali & 6 \\
6 El Salvador & 5 \\
7 Nicaragua & 5 \\
8 Namibie & 5 \\
9 Sénégal & 5 \\
10 Niger & 4 \\
\hline
\end{tabular}

Par groupe de revenu (USD m)

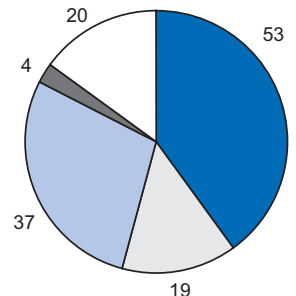

Pays moins avancés

$\square$ Faible reven

$\square$ Revenu intermédiaire,

tranche inférieure

$\square$ Revenu intermédiaire,

tranche supérieure

$\square$ Revenu élevé

$\square$ Non ventilé

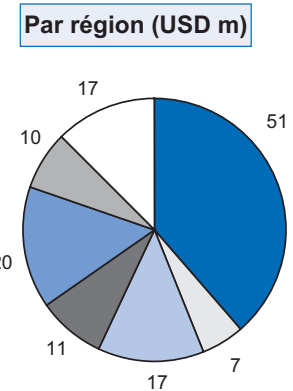

$\square$ Afrique subsaharienn

$\square$ Asie du Sud et centrale

$\square$ Autres pays d'Asie \&

Océanie

$\square$ Moyen-Orient et Afrique

du Nord

$\square$ Amérique latine \&

Caraïbes

$\square$ Europe

$\square$ Non spécifié 


\section{Norvège}

Objectif :

L'objectif premier du programme d'aide de la Norvège est de contribuer à la lutte contre la pauvreté, en fournissant un appui aux stratégies de réduction de la pauvreté des pays partenaires ainsi qu'aux autres stratégies nationales, et de favoriser ainsi la réalisation des OMD (St.prp.nr. 1, 2003-2004, Budget d'aide au développement, approuvé par le Parlement norvégien).

Législation :

Déclaration de politique

Rapport d'orientation $\mathrm{n}^{\circ} 35$ (2003-2004) : Fighting Poverty together - A Comprehensive Development Policy to générale sur l'aide extérieure : the Storting.

Autres déclarations de politique :

Fighting Poverty: The Norwegian Government's Action Plan for Combating Poverty in the South towards 2015 (2002).

Ministres :

Ministre du Développement international, assisté par un secrétaire d’État.

Autres ministres

Ministre des Affaires étrangères, assisté par un secrétaire d’État.

Ministère/organisme de

Ministère des Affaires étrangères (http://odin.dep.no/ud/engelsk).

développement principal :

Autres organismes/ministères: Agence norvégienne de développement international (NORAD) (www.norad.no).

Fredskorpset (Services bénévoles) (www.fredskorpset.no).

Structures de coordination

interministérielles :

Principaux partenaires bilatéraux/bénéficiaires du programme d'aide :

Les sept principaux partenaires bilatéraux sont les suivants: Bangladesh, Malawi, Mozambique, Népal, Tanzanie, Ouganda et Zambie.

On compte également 18 pays partenaires. En Afrique : Angola, Érythrée, Éthiopie, Kenya, Madagascar, Mali, Nigeria et Afrique du Sud. En Asie : Afghanistan, Chine, Timor oriental, Indonésie, Pakistan, Sri Lanka e Viêt-nam. En Amérique centrale : Guatemala et Nicaragua. Au Proche-Orient : Zones sous administration palestinienne.

Principaux domaines de Éducation, VIH/sida, secteur privé et agriculture, développement durable, bonne gouvernance et lutte contre la coopération : corruption, établissement de la paix et développement, santé.

Organes ministériels

consultatifs :

\section{NORVÈGE}

APD bilatérale brute, moyenne 2002-03, sauf indication contraire

\begin{tabular}{|lrrc|}
\hline & & & Variation \\
APD nette & $\mathbf{2 0 0 2}$ & $\mathbf{2 0 0 3}$ & $\mathbf{2 0 0 2 / 0 3}$ \\
Prix courants (USD m) & 1696 & 2042 & $20.4 \%$ \\
Prix constants (2002 USD m) & 1696 & 1775 & $4.6 \%$ \\
En couronnes norv. (millions) & 13544 & 14457 & $6.7 \%$ \\
APD/RNB & $0.89 \%$ & $0.92 \%$ & \\
Aide bilatérale (\%) & $68 \%$ & $72 \%$ & \\
Aide publique nette (AP) & & & \\
Prix courants (USD m) & 45 & 50 & $11.6 \%$ \\
\hline
\end{tabular}

\begin{tabular}{|lr|}
\hline \multicolumn{2}{|c|}{$\begin{array}{c}\text { Dix principaux bénéficiaires } \\
\text { d'APD/AP brute (USD m) }\end{array}$} \\
1 Afghanistan & 65 \\
2 Tanzanie & 57 \\
3 Zones/adm.palestinienne & 52 \\
4 Mozambique & 46 \\
5 Irak & 38 \\
6 Ouganda & 35 \\
7 Serbie \& Monténégro & 33 \\
8 Éthiopie & 33 \\
9 Somalie & 33 \\
10 Zambie & 32 \\
\hline
\end{tabular}

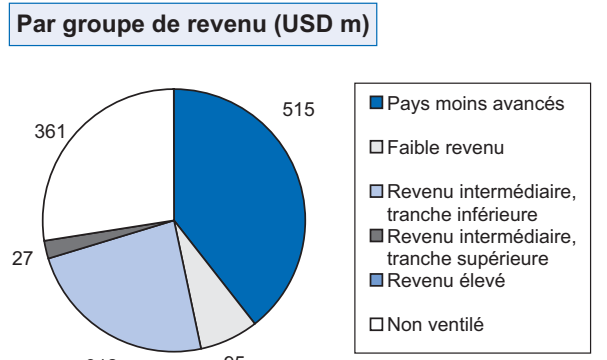

312

Par région (USD $\mathrm{m}$ )

Par secteur

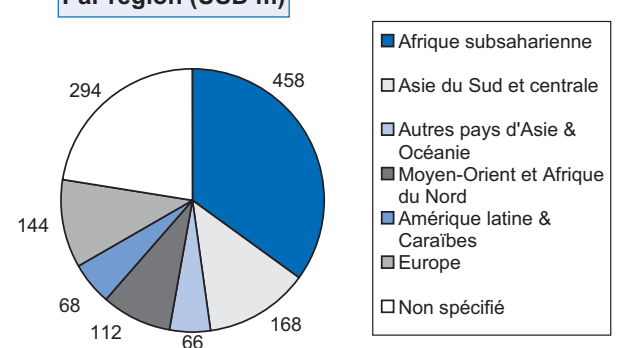

\begin{tabular}{|l|ll|l|l|l|l|l|l|}
\hline & & & & & & \\
\hline
\end{tabular}




\section{Nouvelle-Zélande}

\begin{tabular}{|c|c|}
\hline Objectif : & $\begin{array}{l}\text { Elimination de la pauvreté dans les pays en développement, en collaboration avec les pays partenaires, afin } \\
\text { d'assurer un développement durable et équitable pour les plus démunis (Déclaration de politique } \\
\text { générale 2002). }\end{array}$ \\
\hline Législation : & Sans objet. \\
\hline $\begin{array}{l}\text { Déclaration de politique } \\
\text { générale sur l'aide extérieure : }\end{array}$ & Policy Statement: Towards a Safe and Just World Free of Poverty (2002). \\
\hline \multicolumn{2}{|l|}{$\begin{array}{l}\text { Autres déclarations de } \\
\text { politique : }\end{array}$} \\
\hline Ministres : & Secrétaire d’État aux Affaires étrangères et au Commerce (Aide publique au développement). \\
\hline Autres ministres : & Ministre des Affaires étrangères et du Commerce. \\
\hline $\begin{array}{l}\text { Ministère/organisme de } \\
\text { développement principal : }\end{array}$ & $\begin{array}{l}\text { New Zealand Agency for International Development (NZAID), organe semi-autonome au sein du ministère des } \\
\text { Affaires étrangères et du Commerce (www.nzaid.govt.nz). }\end{array}$ \\
\hline \multicolumn{2}{|c|}{ Autres organismes/ministères : } \\
\hline \multicolumn{2}{|l|}{$\begin{array}{l}\text { Structures de coordination } \\
\text { interministérielles : }\end{array}$} \\
\hline $\begin{array}{l}\text { Principaux partenaires } \\
\text { bilatéraux: }\end{array}$ & $\begin{array}{l}\text { La Nouvelle-Zélande a } 20 \text { partenaires principaux. Onze se trouvent dans le Pacifique : - îles Cook, Fidji, Kiribati, } \\
\text { Niue, Papouasie-Nouvelle-Guinée, Samoa, îles Salomon, Tokelau, Tonga, Tuvalu et Vanuatu, sept sont en Asie - } \\
\text { Cambodge, Chine, Timor oriental, Indonésie, Laos, Philippines et Viêt-nam, et un en Afrique - l'Afrique du Sud }\end{array}$ \\
\hline $\begin{array}{l}\text { Principaux domaines de } \\
\text { coopération: }\end{array}$ & $\begin{array}{l}\text { Développement social, et tout particulièrement : éducation, santé, création de moyens d'existence durables, } \\
\text { égalité homme-femme, gouvernance et droits de l'homme. }\end{array}$ \\
\hline $\begin{array}{l}\text { Organes ministériels } \\
\text { consultatifs : }\end{array}$ & The International Development Advisory Committee (IDAC). \\
\hline
\end{tabular}

\section{NOUVELLE-ZÉLANDE}

\begin{tabular}{|lrrc|}
\hline & & & Variation \\
APD nette & $\mathbf{2 0 0 2}$ & $\mathbf{2 0 0 3}$ & $\mathbf{2 0 0 2 / 0 3}$ \\
Prix courants (USD m) & 122 & 165 & $35.8 \%$ \\
Prix constants (2002 USD m) & 122 & 130 & $6.9 \%$ \\
En dollars de NZ (millions) & 264 & 285 & $8.2 \%$ \\
APD/RNB & $0.22 \%$ & $0.23 \%$ & \\
Aide bilatérale (\%) & $75 \%$ & $78 \%$ & \\
Aide publique nette (AP) & & & \\
Prix courants (USD m) & 1 & 1 & $28.8 \%$ \\
\hline
\end{tabular}

\begin{tabular}{|lc|}
\hline \multicolumn{2}{|c|}{$\begin{array}{c}\text { Dix principaux bénéficiaires } \\
\text { d'APD/AP brute (USD m) }\end{array}$} \\
1 Papouasie-NIle-Guinée & 7 \\
2 Niue & 6 \\
3 Irak & 6 \\
4 Tokelau & 5 \\
5 Salomon, lles & 5 \\
6 Samoa & 5 \\
7 Indonésie & 5 \\
8 Vanuatu & 4 \\
9 Tonga & 4 \\
10 Cook, lles & 3 \\
\hline
\end{tabular}

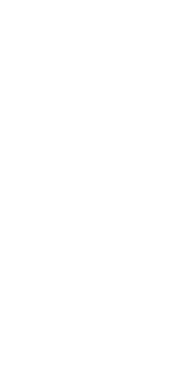

.

Par groupe de revenu (USD m)

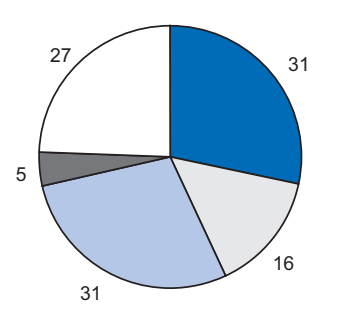
口Pays moins avancés $\square$ Faible revenu
$\square$ Revenu intermédiaire, tranche inférieure $\square$ Revenu intermédiaire, tranche supérieure $\square$ Revenu élevé
$\square$ Non ventilé

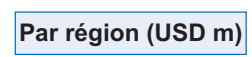

\begin{tabular}{|lll|l|l|l|l|l|}
\hline & & & & \\
\hline
\end{tabular}




\section{Tableau A.1. Pays-Bas}

Objectif :

L'objectif principal est la réduction durable de la pauvreté (Intérêts mutuels, responsabilités mutuelles - Mutual Interests, Mutual Responsibility 2003).

Législation :

Déclaration de politique générale sur l'aide extérieure

Sans objet.

Intérêts mutuels, responsabilités mutuelles : programme néerlandais de coopération pour le développement jusqu'en 2015 (Mutual Interests, Mutual Responsibility: Dutch Development Co-operation en route to 2015) (2003).

Autres déclarations de L'aide en évolution (Aid in Progress) (1995).

politique :

Ministre responsable

Autres ministres :

Ministère/organisme de développement principal :

Ministre de la Coopération pour le Développement.

Ministre des Affaires étrangères.

Direction générale de la coopération internationale (DGIS) au sein du ministère des Affaires étrangères (www.minbuza.nn)

Autres organismes/ministères:

Structures de coordination Conseil de coordination pour les affaires internationales (CORIA)

interministérielles : $\quad$ Comité de coordination pour les affaires européennes (COCO).

Principaux partenaires Les Pays-Bas comptent 36 pays partenaires à long terme. En Afrique : Bénin, Burkina Faso, Cap-Vert, Égypte, bilatéraux/pays bénéficiaires du Érythrée, Éthiopie, Ghana, Kenya, Mali, Mozambique, Rwanda, Sénégal, Afrique du Sud, Tanzanie, Ouganda et programme d'aide : $\quad$ Zambie. En Asie : Afghanistan, Arménie, Bangladesh, Géorgie, Indonésie, Moldavie, Mongolie, Pakistan, Sri Lanka et Viêt-nam. En Amérique latine : Bolivie, Colombie, Guatemala, Nicaragua, Surinam. En Europe : Albanie, Bosnie-Herzégovine, Macédoine. II faut ajouter également les Zones sous administration palestinienne et le Yémen.

Principaux domaines de Les programmes d'aide au développement sont axés sur deux ou trois secteurs seulement. Principaux secteurs coopération : concernés : éducation de base, santé génésique et développement rural au niveau local.

Organes ministériels

consultatifs :

\section{PAYS-BAS}

APD bilatérale brute, moyenne 2002-03, sauf indication contrair

\begin{tabular}{|lrrc|}
\hline & & & Variation \\
APD nette & $\mathbf{2 0 0 2}$ & $\mathbf{2 0 0 3}$ & $\mathbf{2 0 0 2 / 0 3}$ \\
Prix courants (USD m) & 3338 & 3981 & $19.3 \%$ \\
Prix constants (2002 USD m) & 3338 & 3232 & $-3.2 \%$ \\
En euros (millions) & 3542 & 3524 & $-0.5 \%$ \\
APD/RNB & $0.81 \%$ & $0.80 \%$ & \\
Aide bilatérale (\%) & $73 \%$ & $74 \%$ & \\
Aide publique nette (AP) & & & \\
Prix courants (USD m) & 211 & 248 & $17.4 \%$ \\
\hline
\end{tabular}

\begin{tabular}{|lr|}
\hline \multicolumn{2}{|c|}{$\begin{array}{c}\text { Dix principaux bénéficiaires } \\
\text { d'APD/AP brute (USD m) }\end{array}$} \\
1 Congo, Rép. dém. & 178 \\
2 Tanzanie & 117 \\
3 Indonésie & 106 \\
4 Inde & 105 \\
5 Afghanistan & 83 \\
6 Ghana & 81 \\
7 Antilles néerlandaises (AP) & 56 \\
8 Bolivie & 56 \\
9 Aruba (AP) & 52 \\
10 Bangladesh & 51 \\
\hline
\end{tabular}

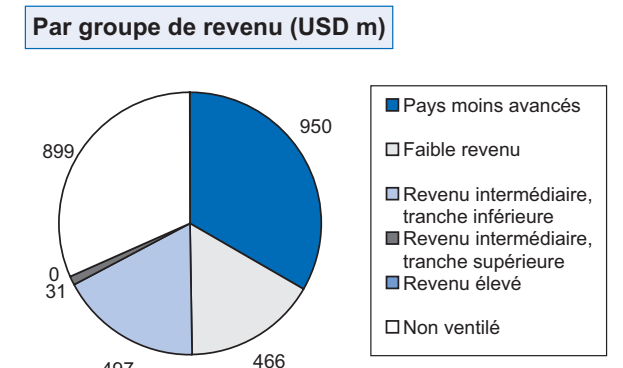

466

Par région (USD $\mathrm{m}$ )

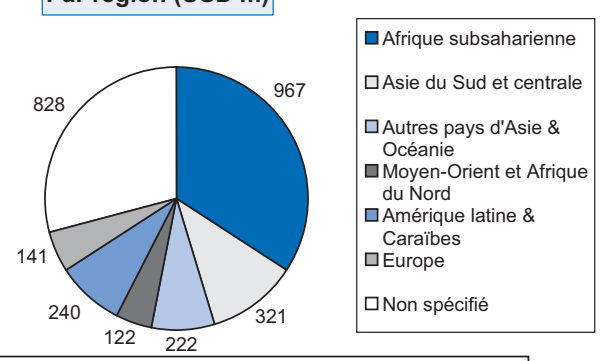

\begin{tabular}{|l|l|l|l|l|l|lll|} 
& & & & & & & & \\
\hline
\end{tabular}




\section{Portugal}

Objectif :

Renforcer la démocratie et l'État de droit, lutter contre la pauvreté, stimuler la croissance économique, favoriser l'intégration régionale et promouvoir un partenariat européen pour le développement humain (Déclaration de politique générale du Portugal).

Législation : Décret-loi 5/2003 du 13 janvier.

Déclaration de politique La coopération en matière de développement à l'aube du XXIe siècle - Document stratégique (The Portuguese générale sur l'aide extérieure : Co-operation for the Incoming 21st Century - Strategy Paper) - Document approuvé par la résolution $\mathrm{n}^{\circ} 43 / 1999$ Autres déclarations de politique :

Ministre responsable Autres ministres

Secrétaire d'État aux Affaires étrangères et à la Coopération, au ministère des Affaires étrangères. Secrétaire d'État aux Affaires européennes.

Secrétaire d'État chargé des Communautés portugaises à l'étranger. Ministre des Finances.

Ministère/organisme de Institute for Portuguese Development Support (IPAD) (www.ipad.mne.pt). développement principal :

Autres organismes/ministères: Ministère des Finances.

Dix-sept ministères et organismes, et plus de 300 municipalités réparties sur 22 districts.

Structures de coordination interministérielles :

Principaux partenaires bilatéraux :

Sept pays partenaires principaux : Angola, Cap-Vert, Timor oriental, Guinée-Bissau, Mozambique, Sao Tomé et Principe.

Principaux domaines de coopération :

Éducation et formation, culture et patrimoine, santé, activités productives et infrastructure, société et institutions, sécurité, assistance financière, aide humanitaire et aide d'urgence.

Organes ministériels

consultatifs :

\section{PORTUGAL}

\begin{tabular}{|lrrc|}
\hline & & & Variation \\
APD nette & $\mathbf{2 0 0 2}$ & $\mathbf{2 0 0 3}$ & $\mathbf{2 0 0 2 / 0 3}$ \\
Prix courants (USD m) & 323 & 320 & $-0.9 \%$ \\
Prix constants (2002 USD m) & 323 & 260 & $-19.4 \%$ \\
En euros (millions) & 342 & 283 & $-17.4 \%$ \\
APD/RNB & $0.27 \%$ & $0.22 \%$ & \\
Aide bilatérale (\%) & $58 \%$ & $57 \%$ & \\
Aide publique nette (AP) & & & \\
Prix courants (USD m) & 33 & 51 & $53.1 \%$ \\
\hline
\end{tabular}

\begin{tabular}{|lr|}
\hline \multicolumn{2}{|c|}{ Dix principaux bénéficiaires } \\
d'APD/AP brute (USD m) \\
1 Timor-Leste & 59 \\
2 Cap-Vert & 26 \\
3 Mozambique & 22 \\
4 Angola & 17 \\
5 Sao Tomé et Principe & 12 \\
6 Guinée-Bissau & 7 \\
7 Irak & 4 \\
8 Sierra Leone & 4 \\
9 Congo, Rép. dém. & 4 \\
10 Bosnie-Herzégovine & 2 \\
\hline
\end{tabular}

APD bilatérale brute, moyenne 2002-03, sauf indication contraire Par groupe de revenu (USD m)
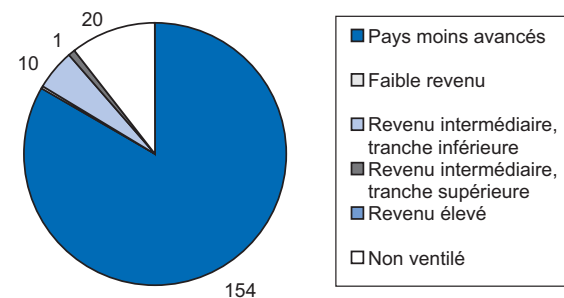

$\square$ Non ventilé
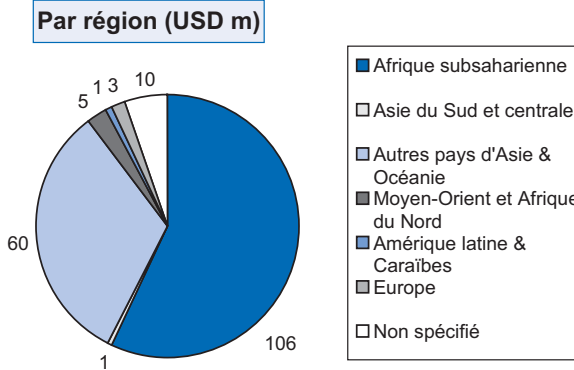

Afrique subsaharienn $\square$ Asie du Sud et centrale $\square$ Autres pays d'Asie \& Océanie $\square$ Moyen-Orient et Afrique du Nord

$\square$ Amérique latine \&

Caraïbes

Europe

$\square$ Non spécifié

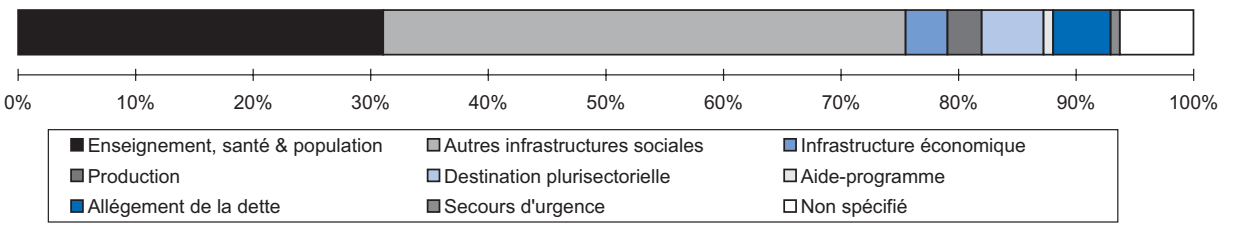




\section{Royaume-Uni}

Objectif :

Législation :

Déclaration de politique générale sur l'aide extérieure

Autres déclarations de politique :

Ministre responsable

Autres ministres :

Ministère/organisme de développement principal :

Autres organismes/ministères: Ministère des Affaires étrangères et du Commonwealth (www.fco.gov.uk).

Structures de coordination interministérielles :

Principaux partenaires bilatéraux :

Principaux domaines de coopération :

Organes ministériels

consultatifs :
Ministère de l'Intérieur (www.homeoffice.gov.uk).

Trésor (www.hm-treasury.gov.uk).

The British Council (www.britishcouncil.org).

Élimination de la pauvreté et promotion d'une croissance économique bénéficiant aux plus pauvres (Eliminating World Poverty, 1997).

Loi sur le développement international (International Development Act - 2002).

(19) Century (1997); Eliminating World Poverty: Making Globalisation Work for the Poor (2000).

Secrétaire d'État au Développement international, assisté d'un ministre et d'un sous-secrétaire d'État parlementaire.

Chancelier de l'Échiquier.

Ministère du Développement international (DFID) (www.dfid.gov.uk).

Le DFID n'a pas de liste officielle des pays prioritaires ; il a toutefois des bureaux ou du personnel dans une quarantaine de pays, dont 20 en Afrique. Les principaux bureaux du DFID se trouvent en Afrique (Ghana, Kenya, Malawi, Mozambique, Nigeria, Sierra Leone, Afrique du Sud, Tanzanie, Ouganda, Zambie et Zimbabwe), en Asie (Afghanistan, Bangladesh, Chine, Inde, Népal, Pakistan et Thaïlande), ainsi qu'en Russie, en Ukraine et aux Barbades.

Santé, éducation, création de moyens de subsistance durables, réforme du secteur de la sécurité, assistance humanitaire, développement du secteur privé.

\section{ROYAUME-UNI}

APD bilatérale brute, moyenne 2002-03, sauf indication contraire

\begin{tabular}{|lrrc|}
\hline & & & Variation \\
APD nette & $\mathbf{2 0 0 2}$ & $\mathbf{2 0 0 3}$ & $\mathbf{2 0 0 2 / 0 3}$ \\
Prix courants (USD m) & 4924 & 6282 & $27.6 \%$ \\
Prix constants (2002 USD m) & 4924 & 5616 & $14.0 \%$ \\
En livres sterling (millions) & 3282 & 3847 & $17.2 \%$ \\
APD/RNB & $0.31 \%$ & $0.34 \%$ & \\
Aide bilatérale (\%) & $71 \%$ & $61 \%$ & \\
Aide publique nette (AP) & & & \\
Prix courants (USD m) & 494 & 698 & $41.3 \%$ \\
\hline
\end{tabular}

\begin{tabular}{|lr|}
\hline \multicolumn{2}{|c|}{ Dix principaux bénéficiaires } \\
d'APD/AP brute (USD m) \\
1 Inde & 346 \\
2 Serbie \& Monténégro & 237 \\
3 Tanzanie & 208 \\
4 Bangladesh & 188 \\
5 Ghana & 130 \\
6 Afghanistan & 115 \\
7 Pakistan & 106 \\
8 Irak & 97 \\
9 Ouganda & 94 \\
10 Afrique du Sud & 87 \\
\hline
\end{tabular}

98

\section{Par groupe de revenu (USD m)}
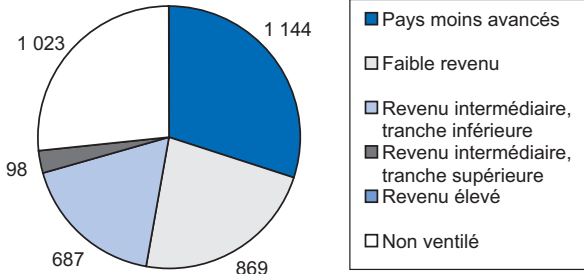

Par région (USD $\mathrm{m}$ )

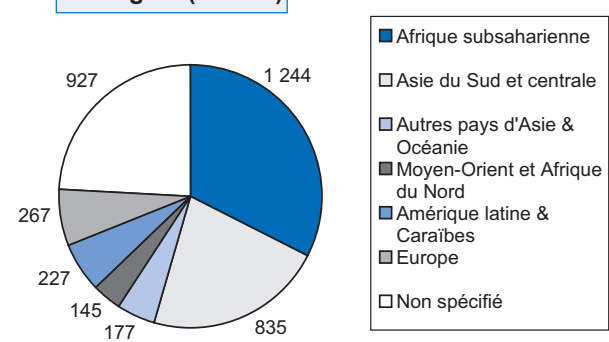

\begin{tabular}{|l|lll|l|l|l|l|}
\hline & & & & & & \\
\hline
\end{tabular}




\section{Suède}

Objectif :

Législation :

Déclaration de politique générale sur l'aide extérieure

Autres déclarations de politique :

Ministre responsable

Autres ministres

Ministère/organisme de développement principal :

Autres organismes/ministères :

Structures de coordination interministérielles :

Principaux partenaires bilatéraux:

Principaux domaines de coopération :

Organes ministériels consultatifs :
Contribuer à créer des conditions propres à encourager les efforts des plus démunis pour améliorer leur qualité de vie (Loi sur le développement mondial - Act on Global Development, 2003).

Sans objet.

Loi sur le développement mondial (2003).

The Rights of the Poor: Our Common Responsibility (1997).

Ministre de la Coopération pour le Développement international, assisté par un secrétaire d'État. Ministre des Affaires étrangères.

Ministre des Finances.

Ministre des Affaires économiques internationales et des Marchés financiers.

Ministre de l'Industrie et du Commerce.

Direction du développement mondial au sein du ministère des Affaires étrangères (www.ud.se).

Agence suédoise de coopération pour le développement international (ASDI) (www.sida.se).

Ministère des Finances (www.sweden.gov.se/govagencies/fiag.htm).

Ministère de l'Industrie, de l'Emploi et des Communications (http://naring.regeringen.se/inenglish/index.htm). Office suédois d'immigration (www.migrationsverket.se/english.htmI).

Institut suédois (www.si.se).

Institut nordique pour l'Afrique (www.nai.uu.se/indexeng.htmI).

La Suède est active dans une centaine de pays.

Assistance humanitaire et prévention des conflits, domaines sociaux, droits de l'homme et gouvernance démocratique, infrastructure, secteur privé et développement urbain. Groupe d'experts sur les questions liées au développement.

\section{SUÈDE}

\begin{tabular}{|lrrc|}
\hline APD nette & $\mathbf{2 0 0 2}$ & $\mathbf{2 0 0 3}$ & $\mathbf{2 0 0 2 / 0 3}$ \\
Prix courants (USD m) & 2012 & 2400 & $19.3 \%$ \\
Prix constants (2002 USD m) & 2012 & 1955 & $-2.8 \%$ \\
En couronnes suéd. (millions) & 19554 & 19388 & $-0.8 \%$ \\
APD/RNB & $0.84 \%$ & $0.79 \%$ & \\
Aide bilatérale (\%) & $63 \%$ & $74 \%$ & \\
Aide publique nette (AP) & & & \\
Prix courants (USD m) & 107 & 127 & $18.9 \%$ \\
\hline
\end{tabular}

\begin{tabular}{|lr|}
\hline \multicolumn{2}{|c|}{$\begin{array}{c}\text { Dix principaux bénéficiaires } \\
\text { d'APD/AP brute (USD } \mathrm{m} \text { ) }\end{array}$} \\
1 Congo, Rép. dém. & 89 \\
2 Tanzanie & 64 \\
3 Mozambique & 51 \\
4 Nicaragua & 37 \\
5 Russie (AP) & 36 \\
6 Afghanistan & 35 \\
7 Zones/adm.palestinienne & 32 \\
8 Bosnie-Herzégovine & 31 \\
9 Serbie \& Monténégro & 30 \\
10 Ouganda & 28 \\
\hline
\end{tabular}
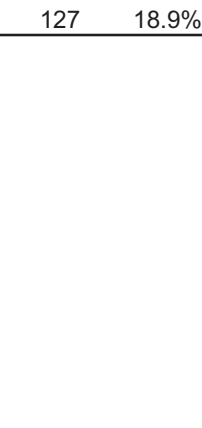

\section{Par secteur}

APD bilatérale brute, moyenne 2002-03, sauf indication contraire

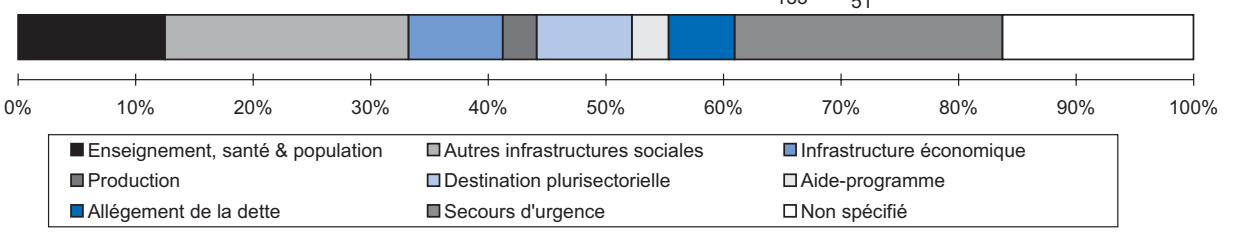




\section{Suisse}

Objectif :

Législation :

sur l'aide extérieure :

Autres déclarations de politique : Rapport sur la politique étrangère (2000).

Message sur la poursuite de la coopération technique et de l'aide financière au bénéfice des pays en développement pour la période 2004-2007 (2003).

Ministre responsable

Autres ministres:

Ministère/organisme de

développement principal :

Autres organismes/ministères:

Structures de coordination interministérielles :

Principaux partenaires bilatéraux : La DDC a 17 pays prioritaires. En Afrique : Bénin, Burkina Faso, Tchad, Niger, Mali, Mozambique et Tanzanie. En Amérique latine : Bolivie, Équateur, Nicaragua, Pérou. En Asie : Bangladesh, Bhoutan, Inde, Népal, Pakistan, Viêt-nam, auxquels s'ajoutent six pays bénéficiant d'un programme spécial : Cuba, Corée du Nord, Zones sous administration palestinienne, Afrique du Sud, Rwanda et Madagascar.

Le SECO a 16 pays prioritaires. En Afrique : Burkina Faso, Égypte, Ghana, Mozambique et Tanzanie. En Asie : Albanie, Bulgarie, Chine, République kirghize, Macédoine, Roumanie, Tadjikistan, Serbie et Monténégro, et Viêt-nam. En Amérique latine : Pérou. II faut ajouter 11 autres pays importants : Azerbaïdjan, Bolivie, Bosnie-Herzégovine, Jordanie, Inde, Indonésie, Maghreb, Russie, Afrique du Sud, Ukraine et Ouzbékistan.

Principaux domaines de coopération :

Pour la DDC : prévention et règlement des conflits, bonne gouvernance, création de revenus, justice sociale, et exploitation durable des ressources naturelles.

Pour le SECO : soutien macroéconomique, promotion de l'investissement, financement des infrastructures, commerce et coopération dans le domaine des technologies " propres ».

Organes ministériels consultatifs : Comité consultatif sur le développement et la coopération au plan international.

\section{SUISSE}

\begin{tabular}{|lrrc|}
\hline & & & Variation \\
APD nette & $\mathbf{2 0 0 2}$ & $\mathbf{2 0 0 3}$ & $\mathbf{2 0 0 2 / 0 3}$ \\
Prix courants (USD m) & 939 & 1299 & $38.4 \%$ \\
Prix constants (2002 USD m) & 939 & 1124 & $19.7 \%$ \\
En francs suisses (millions) & 1462 & 1748 & $19.6 \%$ \\
APD/RNB & $0.32 \%$ & $0.39 \%$ & \\
Aide bilatérale (\%) & $81 \%$ & $73 \%$ & \\
Aide publique nette (AP) & & & \\
Prix courants (USD m) & 66 & 77 & $17.0 \%$ \\
\hline
\end{tabular}

\begin{tabular}{|lr|}
\hline \multicolumn{2}{|c|}{ Dix principaux bénéficiaires } \\
d'APD/AP brute (USD m) \\
1 Serbie \& Monténégro & 41 \\
2 Inde & 24 \\
3 Tanzanie & 22 \\
4 Mozambique & 21 \\
5 Congo, Rép. dém. & 20 \\
6 Burkina Faso & 18 \\
7 Bosnie-Herzégovine & 15 \\
8 Népal & 14 \\
9 Chine & 14 \\
10 Afghanistan & 14 \\
\hline
\end{tabular}

\section{Prarsestar}

APD bilatérale brute, moyenne 2002-03, sauf indication contraire

\section{Par groupe de revenu (USD $\mathrm{m}$ )}

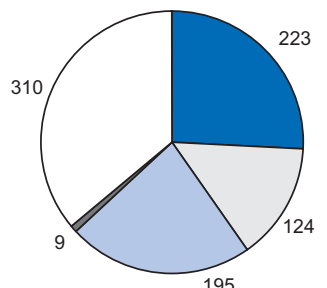

$\square$ Pays moins avancés
$\square$ Faible revenu
$\square$ Revenu intermédiaire,
tranche inférieure
$\square$ Revenu intermédiaire,
tranche supérieure
$\square$ Revenu élevé
$\square$ Non ventilé
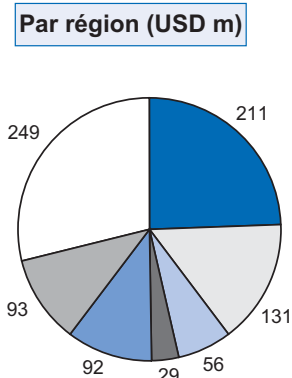

Afrique subsaharienne $\square$ Asie du Sud et centrale

$\square$ Autres pays d'Asie \&

Océanie

Moyen-Orient et Afrique

du Nord

$\square$ Amérique latine \&

Caraïbes

aEurope

$\square$ Non spécifié

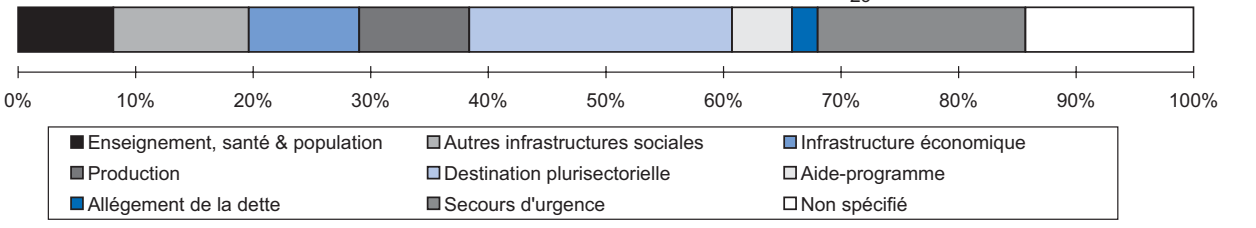





\section{ANNEXE A.2 \\ Rapport sur la gestion des programmes de coopération pour le développement au Mozambique}

\section{Introduction}

Dans le cadre des travaux préparatoires à l'élaboration d'un rapport sur les structures et pratiques de gestion de la coopération pour le développement dans les pays de l'OCDE membres du Comité d'aide au développement (CAD), une mission a été organisée au Mozambique et en Afrique du Sud du 23 février au 3 mars 2004. Cette mission avait un double objectif : i) faciliter une meilleure compréhension des situations complexes et des réalités qui se présentent, dans l'optique de l'action sur le terrain, concernant la gestion et la mise en œuvre des programmes de coopération pour le développement; ii) recueillir, auprès de la plupart des membres du $\mathrm{CAD}$, un ensemble de données de base permettant de dégager des tendances générales.

Les conclusions présentées ici découlent des informations et enseignements fournis lors des entretiens organisés à Maputo ou à Pretoria avec des représentants de 20 pays membres du CAD (sur 22). Dix-huit pays du CAD sont représentés au Mozambique et y sont très actifs, tandis que deux autres gèrent leurs activités dans ce pays depuis leur mission diplomatique à Pretoria, pour l'un d'eux par l'intermédiaire d'un bureau de gestion de projet installé à Maputo ${ }^{1}$. La plupart de ces rencontres se sont déroulées en présence du responsable de la coopération pour le développement, en poste dans la mission locale. Le but premier de l'exercice étant d'en savoir plus sur la manière dont les membres du CAD gèrent leurs programmes d'aide au développement, aucune réunion n'avait été prévue avec des représentants du gouvernement du Mozambique.

La mission était conduite par un membre du Secrétariat de l'OCDE, accompagné d'un consultant engagé pour ce projet. Les participants tiennent à saluer la précieuse contribution du personnel de l'ambassade d'Irlande à Maputo et du Haut Commissariat australien à Pretoria pour l'organisation de la mission.

\section{Contexte du développement au Mozambique : aperçu général}

Avec un revenu annuel de USD 210 par habitant en 2002 (selon l'Atlas de la Banque mondiale), le Mozambique est l'un des pays les plus pauvres de la planète. L'indice du développement humain dans ce pays était, pour la même année, de 170 sur 173 (calculs effectués par le PNUD). Le taux de prévalence du VIH représente $14 \%$ des diagnostics anténataux, un chiffre qui masque cependant d'importants écarts régionaux: dans la province d'Inhambane, par exemple, il atteint $36 \%^{2}$. Peu d'infrastructures subsistent dans 
un pays qui a connu une longue période de guerre civile (jusqu'en 1992), puis des inondations catastrophiques en 2000 et 2001.

Le Mozambique est également l'un des premiers bénéficiaires de l'aide publique au développement (APD) ; il a reçu en 2002, à ce titre, un montant net de USD 1.6 milliard (dont USD 1,1 milliard sous forme d'allègement de la dette).

\section{Principaux enseignements et conclusions}

Nature des programmes de coopération mis en œuvre

- Priorité donnée au Mozambique

Dix-sept membres du CAD ont désigné le Mozambique pays "prioritaire " dans le cadre de leurs activités de coopération pour le développement, le considèrent de facto comme tel, ou lui accordent une place privilégiée dans leur programme d'aide, par exemple en le jugeant admissible à l'octroi de fonds supplémentaires ou à un financement spécial pour des activités hautement prioritaires, comme la lutte contre le VIH/sida. En 2001-2002, le Mozambique était l'un des cinq premiers partenaires de coopération pour onze pays du CAD.

- Ampleur des programmes au Mozambique

L'importance du programme géré et mis en œuvre au bénéfice du Mozambique par chacun des membres du CAD au travers de leurs missions sur le terrain varie entre USD 3 millions environ et plus de USD 90 millions par an, la moyenne étant voisine de USD 32 millions. Ces chiffres ne tiennent pas compte des activités dont la gestion et la mise en œuvre n'est pas assurée par ces missions, notamment l'allègement de la dette, composante majeure de l'APD fournie au Mozambique par certains pays.

\section{- Aspects sectoriels et intersectoriels}

Pour améliorer l'efficience et l'impact de leurs programmes d'aide, quelques membres du CAD ont décidé de concentrer leurs activités sur un petit nombre de secteurs prioritaires. C'est peut-être en partie pour cette raison que la plupart des pays s'intéressent au même groupe de secteurs clés, peut-être au détriment de certains autres domaines, importants pour le développement du Mozambique, dans lesquels le gouvernement partenaire s'efforce d'encourager les donneurs à s'investir davantage (par exemple : la pêche). Quatorze pays interviennent en priorité dans le secteur de la santé (et un quinzième qui axe son action sur la lutte contre le VIH/sida). Pour douze donneurs, l'objectif prioritaire est le développement rural/l'agriculture, onze autres se concentrent sur l'éducation, et neuf sur la bonne gouvernance. Sept pays sont impliqués plus particulièrement dans trois de ces quatre secteurs. Il serait donc possible d'adapter de manière plus judicieuse et plus complète les avantages comparatifs des différents donneurs aux besoins du pays partenaire, afin que tous les aspects importants soient couverts et que le nombre de donneurs dans l'un ou l'autre secteur reste gérable. Dans un seul cas, deux pays se sont entendus sur un partage des responsabilités dans le cadre de leur programme global d'aide, l'un ayant opté pour la santé, l'autre pour l'éducation. Les représentants d'un petit nombre de pays du CAD que nous avons rencontrés se sont déclarés prêts à s'engager dans des partenariats silencieux sur le terrain (en d'autres termes, à fournir des fonds dans un secteur particulier par l'intermédiaire d'un autre donneur déjà activement impliqué dans ce secteur); toutefois, leurs administrations centrales ne sont pas encore disposées à soutenir de telles initiatives. 
Les membres du CAD s'efforcent également d'intégrer différents aspects intersectoriels dans leurs activités. La lutte contre le VIH/sida et l'égalité homme-femme reçoivent l'attention de cinq pays. Parmi les autres domaines privilégiés figurent la gouvernance (trois pays), le développement du secteur privé (deux pays), la décentralisation (deux pays) et l'environnement (un pays).

- Couverture géographique

La plupart des pays membres du CAD axent une partie de leur programme d'aide sur une zone géographique; collectivement, ils couvrent neuf des dix provinces du Mozambique. En règle générale, chaque pays fait porter ses activités sur une, deux ou trois provinces (par exemple: l'Irlande est présente dans les provinces de Niassa et d'Inhambane, tandis que l'Allemagne est active dans les provinces d'Inhambane, de Sofala et de Manica). Toutefois, la répartition globale n'est pas parfaitement homogène: les régions de Niassa et de Maputo bénéficient de l'aide de plusieurs donneurs, alors que certaines provinces très pauvres, comme la région de Zambézia, reçoivent un soutien moins important. Cela s'explique, dans une certaine mesure, par la division progressive et informelle des provinces depuis l'indépendance, en 1975. Dans les provinces où plusieurs donneurs interviennent, les pays peuvent se partager les responsabilités par secteurs (par exemple, dans la province de Sofala, l'Autriche aide principalement au développement rural, tandis que l'Allemagne concentre ses efforts sur l'éducation et l'Italie sur la santé). La plupart des pays du CAD préfèrent maintenir leurs activités à l'échelle de la province, ce qui leur permet de suivre l'impact des mesures prises par le gouvernement du Mozambique et d'exploiter les enseignements qu'ils retirent de l'expérience dans le dialogue mené au niveau national concernant la ligne d'action à adopter. À ce stade, un seul pays projette de réduire ses activités à l'échelle de la province (en l'occurrence, Zambézia).

\section{- Modalités de l'aide}

Les mécanismes de financement conjoint sont bien développés et recueillent un large soutien auprès des bailleurs de fonds présents au Mozambique. En plus du soutien budgétaire global, des fonds sectoriels ont été constitués pour l'agriculture, l'éducation et la santé (où l'on compte en fait quatre fonds sous-sectoriels). Une grande partie de l'aide fournie par certains pays du CAD revêt la forme de contributions communes: un des donneurs rencontrés fournit quasiment les deux tiers de son aide au titre du soutien budgétaire général ; dans un autre cas, la contribution au budget général correspond à $15 \%$ de l'assistance fournie, contre $25 \%$ pour le soutien sectoriel. Actuellement, seuls deux pays représentés à Maputo ne fournissent aucun soutien sectoriel ou budgétaire, mais tous deux envisagent pour l'avenir un soutien sectoriel en commun. Le Mozambique a été choisi comme pays pilote, avec quelques autres, afin de mettre à l'épreuve les modalités d'un financement commun, ce qui a parfois nécessité des procédures exceptionnelles au niveau des administrations centrales. Rares sont les pays en mesure de prendre un engagement ferme sur plusieurs années, ce qui permettrait au Mozambique de planifier à moyen terme sa situation financière et macro-économique. En outre, la plupart des pays participent à ce financement conjoint dans leur propre devise, le Mozambique étant alors confronté aux risques liés aux variations des taux de change, et contraint d'ajuster son budget en conséquence. 
La majorité des membres du CAD contribuant au financement d'une aide sectorielle participent au programme ProAgri, axé sur le secteur agricole. Jugé très bénéfique, ce programme reçoit des contributions de plusieurs pays qui ne participent pas d'ordinaire à ce type d'aide sectorielle. Dans le même temps, toutefois, des frustrations se font jour : d'aucuns estiment en effet que ProAgri améliore surtout les capacités au sein du ministère de l'Agriculture, à Maputo, et n'a eu à ce jour qu'un effet limité dans le reste du pays. L'un des donneurs considère en outre que ses fonds n'ont pas été utilisés de manière appropriée. Un autre a constaté des irrégularités similaires concernant les fonds qu'il met à disposition dans le secteur de l'éducation.

Actuellement, treize membres du CAD (ainsi que l'Union européenne et la Banque mondiale) fournissent un soutien budgétaire général au Mozambique. Ce groupe de donneurs, dénommé " G-15 », constitue désormais l'une des instances les plus actives au Mozambique pour la coordination et l'harmonisation des activités entre les donneurs et pour le dialogue avec le gouvernement du pays partenaire quant aux orientations à adopter - plus active que le Groupe des Partenaires du développement (au sein duquel les donneurs échangent leurs points de vue et leurs idées sur la ligne de conduite à suivre). Certains pays qui ne sont pas en mesure de fournir un soutien financier participent aux réunions générales du G-15 à titre d'observateurs, ce qui atteste l'importance qu'ils accordent à ce groupe. Toutefois, ces observateurs ne peuvent assister à toutes les réunions et n'ont pas accès à certains documents, ce qui limite les possibilités de voir le G-15 devenir légitimement le principal cadre des relations entre donneurs et gouvernement. Par ailleurs, les risques liés à l'apport d'un soutien budgétaire général sont perçus, et acceptés, de manière assez différente selon les pays. Au vu de l'expérience acquise dans les secteurs de l'agriculture et de l'éducation (voir paragraphe précédent), le financement conjoint n'est pas sans risque au Mozambique, comme dans bon nombre de pays en développement. Si certains membres du CAD, convaincus que le soutien budgétaire général peut avoir un impact important sur le développement, assument volontiers ce risque, d'autres se montrent de plus en plus réticents et conditionnent en partie leur soutien à l'évolution positive des normes de gouvernance. Il est difficile de déterminer si les pays qui se sont associés au G-15 à titre d'essai ont défini leur position quant au niveau de risque qu'ils peuvent accepter.

Plusieurs des pays qui agissent principalement dans le cadre de projets s'emploient consciencieusement à donner plus d'impact à leur action en axant cette dernière sur des activités moins nombreuses mais plus concrètes. Cela devrait contribuer en outre à réduire, pour le Mozambique, les frais administratifs liés à la gestion d'un très grand nombre d'activités en faveur du développement ${ }^{3}$.

\section{- Autres activités}

Outre les activités gérées par la mission sur le terrain, l'administration centrale, d'autres agences gouvernementales nationales, ou des autorités régionales et locales peuvent financer des activités de développement au Mozambique. De nombreux pays membres du CAD ont mentionné, au nombre des activités principales auxquelles participent également leurs gouvernements, des programmes régionaux au bénéfice de l'Afrique du Sud, l'assistance bilatérale ou multilatérale, l'assistance humanitaire, l'allègement de la dette, l'octroi de bourses d'études supérieures et le financement conjoint d'activités par des organisations non gouvernementales (ONG). Dans certains pays, le personnel de la mission apporte une contribution modeste à la coordination de ces 
activités ou à leur exécution, par exemple en organisant de temps en temps des tables rondes ou en formulant des observations sur les activités proposées. Pour deux pays membres du CAD, certaines activités sont mises en œuvre au Mozambique indépendamment de la mission locale par un autre ministère ou au niveau infranational, par le biais de représentations implantées dans un pays voisin. Pour de nombreux donneurs, il serait souhaitable d'améliorer la cohérence et la complémentarité des différents niveaux de leurs activités de coopération pour le développement, et de veiller à ce que les bonnes pratiques s'appliquent à tous les secteurs de l'APD.

\section{Présence sur le terrain}

\section{- Représentation sur le terrain}

Les membres du CAD sont nombreux à affirmer que leur programme de coopération pour le développement constitue la première justification de leur représentation au Mozambique. $\mathrm{Si}$, par le passé, les missions diplomatiques étaient souvent gérées par un responsable du développement du niveau d'un chargé d'affaires, plusieurs pays ont relevé le niveau de leur représentation sur place : quinze donneurs ont désormais un ambassadeur/haut commissaire en poste à Maputo (pouvant être accrédité auprès d'autres pays également). Dans quatre autres cas, l'ambassadeur/haut commissaire accrédité auprès du Mozambique réside à Harare ou à Pretoria. Enfin, un pays présente une configuration inhabituelle, le chargé d'affaires à la tête de la mission diplomatique n'étant pas tenu de rendre compte à un ambassadeur/haut commissaire non résident.

Sur les 18 membres du CAD représentés au Mozambique, seules huit missions sur le terrain font partie intégrante de l'ambassade/haute commission. Dans chacun de ces cas, le ministère des Affaires étrangères joue un rôle prépondérant dans la gestion ou la coordination du programme de coopération pour le développement ; par ailleurs, la structure globale du système de coopération mis en place par le pays ne comporte pas d'agence d'exécution distincte. S'agissant des dix autres pays, la mission est implantée dans des locaux séparés, l'organe d'exécution chargé du programme de coopération pour le développement est séparé de l'ambassade ou cette dernière se trouve dans un autre pays.

Maintenant qu'un nombre très important de pays ont un ambassadeur/haut commissaire résidant au Mozambique, la coordination et le dialogue sur les orientations se situent à deux niveaux : celui du chef de mission et celui du responsable de la coopération. La cohérence des messages émis par les membres du CAD et des approches qu'ils adoptent peut se trouver renforcée si les personnes occupant ces postes appartiennent au même ministère et travaillent à proximité géographique l'une de l'autre. Si tel n'est pas le cas, le risque de divergences et d'une moins bonne transmission de l'information peut s'accroître. Les pays n'ayant pas d'ambassadeur/haut commissaire résidant au Mozambique peuvent désormais avoir plus de difficulté à se faire entendre dans les débats menés au plus haut niveau politique.

- Rôles et responsabilités des missions sur le terrain

Les projets et les programmes que les membres du CAD soutiennent au Mozambique sont globalement conformes aux orientations générales et aux approches de ces pays, les personnels des administrations centrales et des missions de terrain ayant tous un rôle à jouer dans la définition et la mise en œuvre des activités. Les différences sont cependant 
considérables entre les pays en ce qui concerne les responsabilités précises des missions sur le terrain et les délégations de compétence sur place. La moitié environ des pays du CAD ont des systèmes plutôt centralisés: la mission exécute les décisions prises par l'administration centrale, avec une marge de manœuvre très limitée, voire inexistante, pour modifier les programmes ou leur financement. À l'inverse, certaines missions conçoivent et mettent en œuvre des programmes, sous réserve que leur gouvernement donne son approbation, et peuvent apporter certaines modifications quant au financement dans les limites définies par le pays selon un processus itératif associant les personnels du ministère et du terrain. Dans deux cas, plusieurs entités interviennent pour la mise en œuvre du programme de coopération, l'une étant très décentralisée, tandis que les autres doivent consulter régulièrement leur administration centrale, ce qui peut rendre plus difficile la coordination interne sur le terrain. Dans plusieurs pays, l'évolution vers une plus forte décentralisation est récente, voire toujours en cours. Dans la plupart des cas, ces processus entraînent des changements dans les activités de la mission - les rapports de routine aux administrations centrales, notamment, sont moins nombreux - mais ne nécessitent pas de modification de son effectif global.

S'agissant des montants nécessaires au financement de nouvelles activités sur le terrain, quatre pays ont indiqué qu'aucune approbation n'était possible sans en référer à leur capitale. Par contraste, huit chefs de mission ont déclaré qu'ils pouvaient prendre ce type de décision, en principe avec un plafond, dans les limites du cadre général défini pour le pays. Les plafonds peuvent varier considérablement, deux pays ayant signalé qu'aucune limite n'avait été fixée, et une autre mission ayant indiqué que seuls des montant ne dépassant pas USD 350000 pouvaient être approuvés. Toutefois, même lorsque la mission est habilitée à donner son accord pour des sommes importantes, elle peut être tenue de consulter son administration centrale sur certaines activités particulières, comme le soutien budgétaire général, l'aval nécessaire émanant alors du ministre ou du gouvernement. Dans au moins un cas, le plafond a été relevé récemment, en vue d'un transfert d'autorité à la mission sur le terrain. Enfin, les chefs de mission de certains pays disposent d'un montant limité qu'ils peuvent choisir d'affecter à des objectifs liés au développement : activités culturelles, financement d'ONG locales, programmes de dons à petite échelle. Ces fonds représentent chaque année des montants compris approximativement entre USD 12000 et USD 675000.

\section{Questions de personnel}

\section{- Effectifs}

Malgré l'intérêt que suscite la question des effectifs assurant la gestion et la mise en œuvre des programmes de coopération pour le développement, on dispose sur le sujet de peu d'informations fiables et se prêtant à des comparaisons. Différents facteurs rendent difficile la compilation de ces données. Chaque programme a ses caractéristiques propres, et certaines activités peuvent nécessiter moins de personnel que d'autres. L'ampleur du soutien et des mesures de facilitation émanant du ministère peut varier, tout comme la fréquence des rapports à soumettre aux responsables des administrations centrales, ainsi que la nécessité de consulter ces derniers et de discuter avec eux des problèmes qui se posent. Certains donneurs confient à des organismes extérieurs des activités dont d'autres préféreront se charger en interne, comme le suivi régulier des activités et l'élaboration de rapports, la sélection et la formation du personnel, ou la recherche. Certains pays laissent 
à leur mission la liberté de choisir le personnel affecté sur le terrain, dans les limites de l'enveloppe budgétaire accordée, y compris en faisant appel, le cas échéant, à des étrangers ou à du personnel local pour des postes de catégorie professionnelle. D'autres sont au contraire soumis à de fortes contraintes, chaque création de poste devant être approuvée par plusieurs entités gouvernementales aux objectifs contradictoires. Certains pays peuvent engager du personnel en le rémunérant sur les fonds affectés au programme (c'est-à-dire des montants qui auraient pu servir à financer des activités de développement) alors que d'autres n'y sont pas autorisés. Enfin, le nombre d'heures prestées par le personnel peut varier lui aussi, en fonction de la durée légale du temps de travail hebdomadaire et des congés.

En dépit de ces difficultés intrinsèques, nous avons tenté d'évaluer le nombre de professionnels ${ }^{4}$ auxquels les pays du CAD font appel sur le terrain pour gérer et mettre en œuvre leurs programmes d'aide au Mozambique. Plusieurs pays disposent au total de six professionnels, voire moins, tandis que deux autres en comptent plus de soixante-quinze (voir graphique A.2.1). Si la moyenne est de 21, les chiffres se situent entre 10 et 15 dans la plupart des cas. Un pays dispose actuellement de 40 conseillers à long terme au Mozambique. Enfin, plusieurs donneurs ont signalé le retrait progressif de leurs conseillers à long terme sur le terrain, dans le cadre d'une évolution globale de leur action visant à privilégier une approche davantage fondée sur des programmes que sur des projets.

Un nombre important de pays membres du CAD jugent que les effectifs dont ils disposent sur le terrain ne leur permettent pas de participer à l'ensemble des mécanismes de coordination entre donneurs qui existent aujourd'hui au Mozambique, ni de se tenir au fait des développements qui interviennent dans leurs différents domaines d'intérêt. Certains ont reconnu que, pour cette raison, le personnel consacre trop de temps à des réunions à Maputo, risquant ainsi de se déconnecter de la réalité dans les régions pauvres et isolées du pays. Plusieurs pays ont souligné également que la participation accrue aux mécanismes de financement conjoint nécessite des qualifications différentes: les

\section{Graphique A.2.1. Importance des programmes et importance des effectifs au Mozambique}

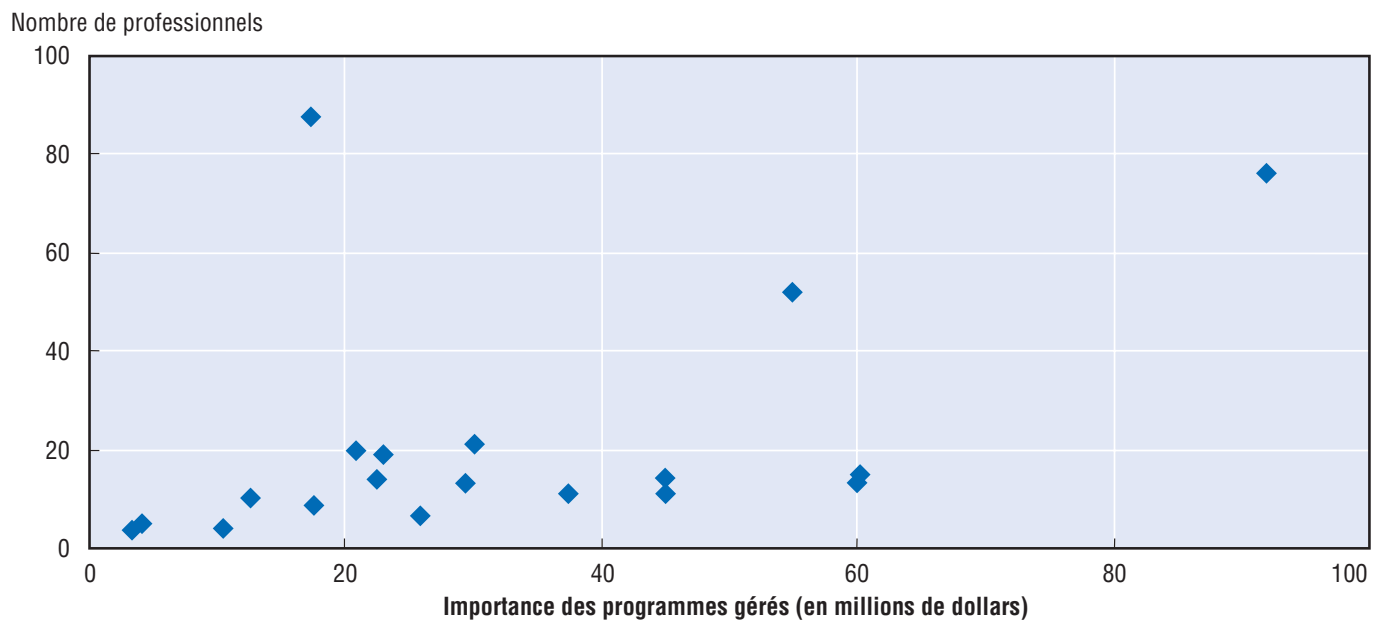

Source: Informations fournies par les pays membres du CAD. 
compétences opérationnelles deviennent moins essentielles, contrairement à la capacité de coopérer avec les autres parties prenantes, de les influencer, et d'analyser les politiques.

Les différences sont manifestes, également, en ce qui concerne les effectifs prévus dans les administrations centrales pour fournir un soutien au personnel de terrain. Dans un des cas examinés, quatre agents sont affectés, dans leur capitale, au seul programme d'aide au Mozambique, alors que dans un autre pays, deux personnes sont seules à suivre les activités menées par le donneur concerné pour l'ensemble de l'Afrique. La plupart des administrations centrales comptent au minimum un responsable chargé à temps partiel de l'aide au Mozambique, et au maximum deux responsables à plein-temps. De nombreux pays ont indiqué par ailleurs que les services des administrations centrales qui doivent traiter de plusieurs domaines sont amenés à s'occuper de temps en temps du programme de coopération pour le Mozambique.

Les informations recueillies dans le cadre du présent exercice permettent de comparer sommairement les volumes financiers gérés sur le terrain, par professionnel, au nom des différents membres du CAD. Dans neuf pays, une personne gère en moyenne entre USD 700000 et USD 1.5 million par an (voir graphique A.2.2), quelle que soit l'ampleur du programme. Pour quatre pays, un professionnel gère environ USD 4 millions ou plus chaque année, ce chiffre n'étant que de USD 200000 à l'autre extrémité de la fourchette. Sur l'ensemble des membres du CAD présents au Mozambique, chaque professionnel gère en moyenne quelque USD 1.5 million par an.

- Politiques relatives au personnel local

La plupart des pays membres du CAD reconnaissent que le personnel local apporte une valeur ajoutée à leur action sur le terrain : ces personnes représentent souvent la mémoire institutionnelle de la mission et apportent des connaissances que le personnel

\section{Graphique A.2.2. Programmes gérés au Mozambique, par professionnel}

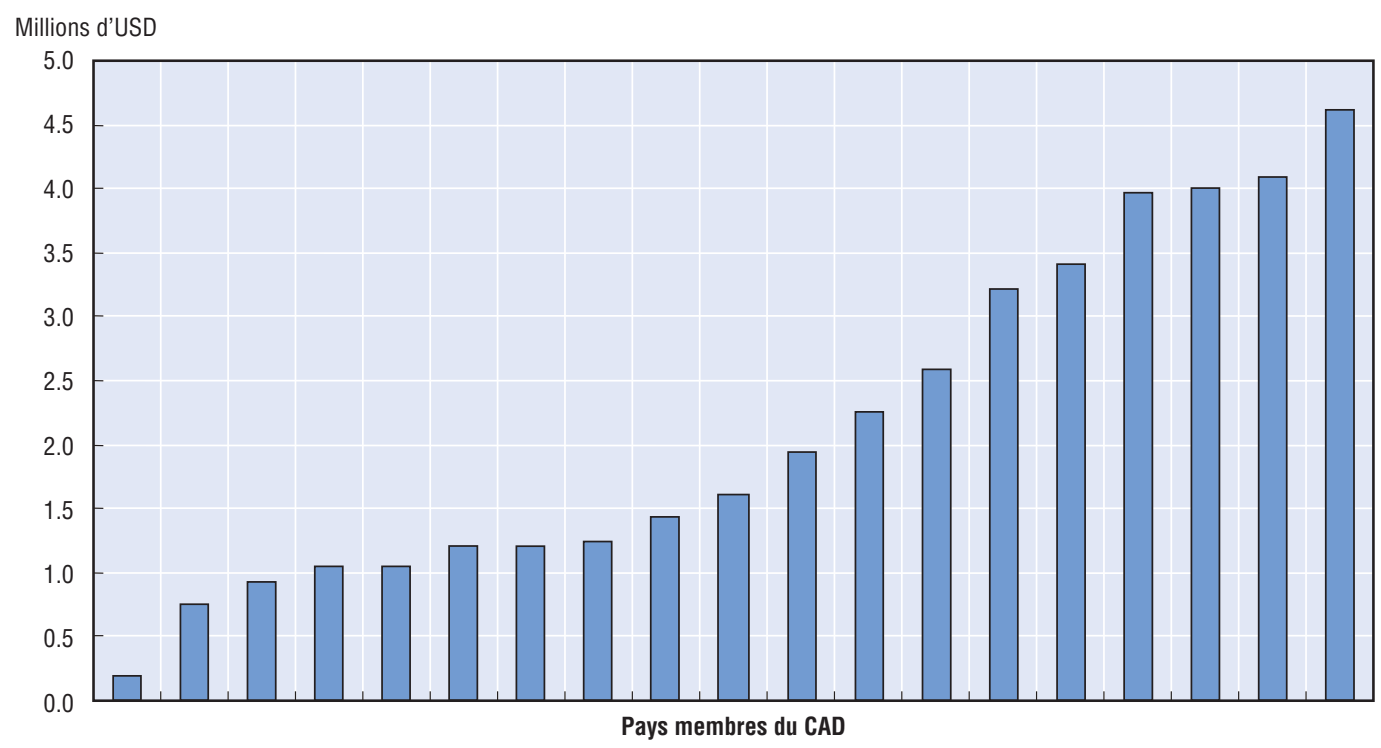

Source: Informations fournies par les membres du CAD. 
international ne peut acquérir facilement. La majorité des pays peuvent recruter du personnel sur place et optent pour cette formule ; deux seulement ont déclaré être dans l'impossibilité de le faire. Par ailleurs, les plans de décentralisation de certains pays consistent principalement à faire appel de plus en plus souvent à du personnel local pour des postes de catégorie professionnelle, voire pour des fonctions d'encadrement, dans les missions de terrain. Si les pays ont indiqué, dans leur majorité, n'avoir mis en place aucune politique particulière pour favoriser les perspectives de carrière à long terme pour le personnel recruté sur place, ils ont néanmoins mentionné la possibilité de suivre une formation, au Mozambique ou dans leur propre administration. En revanche, deux pays ont adopté une politique en la matière, l'un d'eux proposant des contrats permanents assortis d'une formation complète, d'un plan de retraite, et de possibilités de transferts dans d'autres missions. Quelques pays appliquent, à titre formel ou informel, une politique visant à encourager le personnel local à retourner, à terme, dans la société mozambicaine, d'autres doutant au contraire qu'il s'agisse d'une proposition réaliste compte tenu de la baisse de salaire considérable que cela entraînerait probablement. Dans le secteur de la santé, un nombre croissant de pays ont signé un protocole d'accord avec le gouvernement mozambicain, s'engageant à éviter de recruter des fonctionnaires locaux.

Le recours de plus en plus fréquent à du personnel recruté localement, au Mozambique comme dans d'autres grands pays partenaires, a influé sur la décision prise par les organismes de développement de plusieurs membres du CAD non anglophones d'adopter l'anglais comme langue de travail. Dans le même temps, des représentants de pays du CAD non anglophones ont souligné l'importance d'une utilisation active de la langue nationale du pays partenaire et du maintien de la diversité linguistique afin de fournir des bases conceptuelles de remplacement pour l'analyse et la discussion des problèmes.

Quelques donneurs ont mis en place une politique de lutte contre le VIH/sida sur le lieu de travail. Celle-ci consiste généralement à sensibiliser le personnel, ainsi qu'à proposer - gratuitement et dans le respect de la confidentialité - des conseils, des tests de dépistage et un traitement; toutefois, certains se sont dits préoccupés de ce que le traitement des membres de la famille soit actuellement insuffisant. Plusieurs pays sans politique formelle dans ce domaine ont cependant prévu des plans d'assurance-maladie généraux permettant de couvrir certains aspects du traitement. Certains pays, estimant que leurs effectifs recrutés localement sont trop limités pour justifier une politique spécifique, sont d'avis que les situations peuvent être - et sont parfois - réglées au cas par cas. Plusieurs ont indiqué que les administrations centrales s'emploient actuellement à élaborer des politiques applicables à l'échelle des organismes de coopération.

\section{- Échanges entre les administrations centrales et le terrain}

L'intensification du dialogue, sur le terrain, avec le gouvernement du pays partenaire et les autres donneurs, ainsi que la décentralisation accrue des responsabilités - qui incombent plus fréquemment aux missions - mettent en lumière la nécessité de disposer, sur place, d'effectifs expérimentés et qualifiés. Les systèmes adoptés par la plupart des membres du CAD permettent l'envoi sur le terrain de personnels des administrations centrales, lesquels font ensuite bénéficier le siège, ou un autre pays en développement, des connaissances et de l'expérience qu'ils ont acquises au cours de leur mission. Toutefois, dans quelques pays du CAD dont les méthodes de gestion restent plutôt centralisées, rien n'est prévu pour permettre la rotation du personnel entre le siège et le terrain. Les 
programmes de coopération pour le développement peuvent alors être gérés sur le terrain par des diplomates en poste à l'ambassade ou par des consultants recrutés sur place à cette fin. Ni l'une ni l'autre de ces formules ne renforce l'expertise du personnel en matière de développement dans le cadre des systèmes de coopération des donneurs, ni ne facilite une décentralisation plus importante des responsabilités en faveur des missions de terrain.

\section{Questions de gestion}

- Enveloppes budgétaires des pays

La plupart des membres du CAD agissent, en ce qui concerne le Mozambique, avec une enveloppe budgétaire globale à partir de laquelle sont financées la majorité des activités gérées localement. Ces budgets peuvent porter sur plusieurs années, les niveaux de financement des années à venir n'ayant qu'une valeur indicative. Dans d'autres pays, chaque activité doit être approuvée, les engagements financiers nécessaires étant pris pour la durée de cette activité. Dans le cadre de ce processus, un portefeuille d'activités peut être établi, qui constitue le programme au bénéfice du pays. Aucune de ces formules ne garantit au pays partenaire les engagements financiers fermes et pluriannuels que le CAD inscrit désormais au nombre des bonnes pratiques.

- Coût de la mise en œuvre du programme

N'étant pas en mesure de chiffrer avec précision le coût de la gestion de leur programme de coopération pour le développement au Mozambique, la majorité des membres du CAD se trouvent dans l'impossibilité d'indiquer si la mise en œuvre de ce programme s'effectue avec plus ou moins d'efficacité par rapport à l'année précédente ou à d'autres pays. La plupart des pays peuvent communiquer le montant des frais administratifs de leur ambassade, qui constitue une indication approximative, mais ce chiffre tient compte également d'activités sans rapport avec le développement. Par ailleurs, les rémunérations des personnels en mission, en déplacement ou en formation proviennent parfois de budgets séparés des administrations centrales, et les traitements de certains agents affectés au programme de développement peuvent être financés sur les fonds attribués au programme. Quatre pays ont chiffré leurs coûts administratifs, lesquels représentent $5.1 \%$ des fonds consacrés au programme dans un cas, et $6.2 \%$ dans un autre. Pour trois d'entre eux, la mission occupe des locaux distincts de l'ambassade/de la haute commission et, par conséquent, gère séparément ses coûts administratifs. Si quelques pays jugent peu onéreuse la gestion de leurs missions, deux ont constaté au contraire une augmentation de leurs frais en raison de transferts vers des locaux séparés.

\section{- Systèmes de communication}

Pour tous les membres du CAD, les systèmes de communication comprennent le courrier électronique et des lignes téléphoniques fixes pour communiquer avec le siège de leurs gouvernements. Si l'on fait abstraction des bandes passantes trop étroites et des virus (sources de préoccupation pour plusieurs donneurs), une connexion par satellite améliore considérablement les possibilités d'interaction des administrations centrales et des missions, notamment en permettant l'accès à l'Intranet. La majorité des pays disposent désormais de communications par satellite, un dispositif mis en place pour deux d'entre eux dans le cadre du processus de décentralisation. Les pays qui ne sont pas encore dotés de ce système sont manifestement désavantagés, à deux égards: d'une part, les 
fournisseurs de services locaux ne sont pas toujours fiables, d'autre part, le téléchargement des messages électroniques nécessite plusieurs appels téléphoniques quotidiens à leur ministère. Les missions dont les locaux sont installés dans les ambassades ou les hautes commissions bénéficient généralement de moyens de communication plus efficaces, mais les limitations d'accès aux systèmes protégés peuvent restreindre l'efficacité des communications avec l'administration centrale pour les membres du personnel dont le niveau d'habilitation est insuffisant (notamment les ressortissants étrangers). Enfin, s'agissant des communications sur l'ensemble du territoire mozambicain, le téléphone portable est souvent jugé plus pratique et plus fiable que le téléphone fixe.

Les missions de deux pays sont équipées d'un système de vidéoconférence, et un troisième pourrait y avoir accès grâce à un nouveau système de satellite, dont l'installation devrait avoir lieu prochainement. Trois autres pays ont indiqué qu'ils utilisaient occasionnellement les installations de vidéoconférence dans les locaux de la Banque mondiale, à l'université ou dans le bâtiment principal de leur ambassade. Cette technologie, utilisée principalement par les pays qui délèguent des responsabilités importantes à leurs missions et dont le fuseau horaire ne diffère pas sensiblement de celui du Mozambique, sert différents objectifs, parmi lesquels: entretiens de candidats, réunions hebdomadaires avec des cadres de l'administration centrale, participation à des réunions de commissions d'évaluation des projets, communication avec les bureaux régionaux.

\section{Partenariat}

- Stratégies des pays

La majorité - mais pas la totalité - des membres du CAD ont élaboré leur stratégie propre pour la mise en œuvre de leur programme au Mozambique. Dans deux cas, il s'agit moins, d'un point de vue technique, de stratégies que de protocoles d'accord signés avec le gouvernement du pays partenaire. Bien que tous les donneurs aient déclaré que la stratégie de leur pays est conforme à la stratégie nationale de lutte contre la pauvreté (plan du Mozambique couvrant les années 2001-2005), les stratégies des donneurs portent sur des périodes allant de 2 à 6 ans et sont déterminées principalement en fonction de leurs procédures nationales. Il s'agit essentiellement de documents internes, dont un bref résumé peut cependant être diffusé. Certains pays ont opté clairement pour la publication de leur stratégie, voire de sa traduction dans la langue du pays partenaire.

\section{- Consultations de haut niveau}

En dépit des contacts réguliers qui se sont instaurés durablement, grâce à une riche palette de mécanismes de coordination, entre les donneurs et le gouvernement du Mozambique, de nombreux pays membres du CAD ont maintenu la pratique des consultations bilatérales de haut niveau. Celles-ci sont annuelles dans un petit nombre de cas seulement : la tendance est plutôt à la tenue de consultations en fonction des besoins, par exemple lorsqu'un pays met au point une nouvelle stratégie ou lorsqu'un ministre effectue une visite au Mozambique. Plusieurs pays ont indiqué que, lors de consultations de haut niveau (de même qu'aux réunions du Groupe consultatif), leur délégation est conduite par un haut responsable de l'administration centrale. Un petit groupe de pays a révélé la suppression totale de ces consultations, certains expliquant ce choix par le fait 
que les réunions du groupe de soutien budgétaire G-15 fournissent l'occasion de contacts réguliers avec le gouvernement partenaire.

- Suivi et évaluation des performances

Les pays membres du CAD ont des conceptions différentes du suivi de l'impact de leurs activités au Mozambique. Pour certains d'entre eux, et notamment ceux dont le système de gestion est centralisé, les demandes officielles de suivi sont peu nombreuses, ce qui peut parfois s'avérer frustrant pour le personnel de terrain. Il arrive qu'aucun cadre logique n'ait été prévu pour quelque activité que ce soit, le suivi se résumant alors à une collecte rudimentaire d'informations anecdotiques. Quelques pays, en revanche, ont mis $\mathrm{au}$ point des systèmes complexes de gestion stratégique: les résultats concernant différentes activités bien précises sont alors suivis de près et rassemblés, de manière à évaluer l'impact global du programme mis en œuvre par le pays en question concerné. Dans certains cas, les indicateurs sont choisis en fonction de l'activité et des objectifs d'ensemble du pays. Dans d'autres, on s'efforce consciencieusement d'utiliser pour le suivi, dans toute la mesure du possible, les systèmes disponibles dans le pays partenaire. Autre tendance constatée : le recours de plus en plus fréquent à l'informatique lorsque des systèmes de communication appropriés sont disponibles, pour le suivi et l'évaluation de toutes les activités financées par un organisme dans le monde.

Un petit nombre de membres du CAD seulement procèdent à des évaluations complètes de leurs programmes ; il s'agit généralement de donneurs dont les programmes ne concernent qu'un petit nombre de pays. Lorsque de nombreux pays sont en cause, le plan d'évaluation annuel est déterminé par les administrations centrales, même si les missions peuvent influer sur le choix des évaluations à effectuer. Il est de plus en plus fréquent que ces évaluations couvrent un ensemble d'activités menées au Mozambique ou une série d'activités similaires au bénéfice de plusieurs pays en développement. Les évaluations conjointes sont de plus en plus nombreuses au Mozambique, en raison des possibilités de financement conjoint d'activités, en particulier dans le cadre des contributions au budget général, qui ont la faveur d'un nombre croissant de bailleurs de fonds et représentent une part plus importante de l'ADP. Par ailleurs, certains donneurs ayant décidé d'axer davantage leurs activités sur les résultats et sur le maintien de critères qualitatifs rigoureux, le rôle premier des évaluations est désormais de dégager des enseignements et d'en permettre la mise en commun.

Peut-être le développement des financements conjoints a-t-il également pour conséquence l'intérêt accru des auditeurs ou des sociétés d'audit des membres du CAD pour les activités que les donneurs financent au Mozambique. Toutefois, les missions de commissaires aux comptes nationaux au Mozambique pèchent par un certain manque de coordination; il reste beaucoup à faire dans le sens d'une plus grande harmonisation de ces activités, y compris en ce qui concerne les normes employées, comme cela existe maintenant pour les évaluations.

\section{Notes}

1. Plusieurs donneurs non membres du CAD - dont le Brésil, la Chine et l'Islande - sont également actifs au Mozambique.

2. ONUSIDA/OMS, Le point sur l'épidémie du sida, décembre 2003, OMS, Genève. 
3. En moyenne, 845 activités nouvelles en matière de développement ont été lancées chaque année au Mozambique entre 1999 et 2001.

4. Pour cet exercice, les catégories de personnel suivantes n'ont pas été prises en compte : bénévoles, assistants administratifs, secrétaires, chauffeurs, gardes de sécurité, personnel d'entretien et jardiniers. 



\section{ANNEXE A.3 \\ Les statistiques du CAD : introduction}

\section{Portée}

Les statistiques du CAD ont pour objet de mesurer les apports de ressources internationales en faveur du développement ou de l'amélioration du niveau de vie dans les pays en développement ou en transition. Leur conception est influencée par deux types de facteurs :

- Au plan technique: principalement les conventions utilisées dans les statistiques relatives aux balances des paiements.

- Au niveau des orientations: essentiellement par le désir des membres du CAD de montrer, aux fins de comparaison, la pleine étendue de leurs efforts respectifs en faveur du développement.

\section{Types d'apports}

Les apports de ressources peuvent provenir du secteur privé ou du secteur public, et être consentis aux conditions du marché ou à des conditions de faveur. Les données collectées sont réparties dans les grandes catégories ci-après :

Aide publique au développement (APD) et aide publique - Apports du secteur public du pays donneur, présentant les caractéristiques suivantes :

- Ils visent en premier lieu à favoriser le développement économique et l'amélioration du bien-être dans le pays partenaire.

- Ils se présentent sous la forme de dons ou de prêts accordés à des conditions libérales.

L'expression " aide publique au développement " (APD) est appropriée si l'aide en question est destinée à un pays en développement figurant dans la partie I de la Liste des bénéficiaires établie par le CAD (voir ci-après) ou à un organisme multilatéral actif dans le domaine du développement ; l'« aide publique » (AP) s'adresse aux pays en transition ou à un organisme multilatéral dont l'action vise principalement ces pays.

L'APD/AP comprend les coûts que représentent pour les donneurs l'assistance-projet et l'assistance-programme, la coopération technique, les annulations de dettes non encore répertoriées comme $\mathrm{APD}$, les frais de subsistance des réfugiés pendant la première année de leur séjour dans un pays donneur, les coûts imputés des étudiants, l'aide alimentaire, l'aide d'urgence et les dépenses administratives connexes.

Autres apports de fonds publics - Il s'agit i) de dons ou de prêts provenant du secteur public qui ne sont pas spécifiquement affectés au développement ou à l'amélioration du 
bien-être (octroyés par exemple pour des raisons commerciales) ; ii) de prêts émanant du secteur public pour favoriser le développement ou l'amélioration du bien-être, mais dont les conditions ne sont pas suffisamment avantageuses pour qu'ils puissent entrer dans la catégorie APD (voir également la section suivante).

Apports de fonds privés aux conditions du marché - Ils concernent les apports de ressources attribués par le secteur privé d'un pays donneur pour des raisons commerciales. Sont compris sous cette appellation : l'investissement direct étranger, les prêts bancaires et l'achat d'obligations ou de titres du pays en développement par des sociétés ou des particuliers des pays donneurs.

Dons privés - Fonds émanant d'organisations non gouvernementales au titre de l'aide au développement ou à l'amélioration du bien-être.

\section{Note sur les prêts consentis à des conditions libérales}

Pour répondre aux critères de l'APD/AP, un prêt doit s'assortir d'un élément de libéralité d'au moins 25 pour cent, calculé sur la base d'un taux fixe d'actualisation de 10 pour cent. À titre indicatif, on trouvera ci-après quelques exemples de prêts qui satisfont de justesse à ces critères, avec un "élément de libéralité » d'un peu plus de 25 pour cent :

- Prêt sur six ans, remboursements annuels, taux d'intérêt de $0.75 \%$.

- Prêt sur dix ans, remboursements annuels, taux d'intérêt de $3.5 \%$.

- Prêt sur vingt ans, remboursements annuels, taux d'intérêt de $5.5 \%$.

- Prêt sur dix ans, remboursements annuels avec une période de grâce de cinq ans, taux d'intérêt de $5 \%$.

- Prêt sur quinze ans, remboursements annuels avec une période de grâce de cinq ans, taux d'intérêt de $5.75 \%$.

\section{Exclusions}

La contribution potentielle des transactions ci-après au développement est jugée insuffisante pour qu'elles puissent être prises en compte dans les statistiques du CAD :

- Prêts remboursables en un an.

- Dons et prêts octroyés à des fins militaires.

- Transferts financiers à des particuliers (par exemple: pensions, envois de fonds des travailleurs expatriés).

Les ressources provenant de pays partenaires, comme les investissements effectués par des ressortissants de ces pays chez les donneurs, ne sont pas pris en considération non plus. Toutefois, les remboursements par les partenaires des prêts qui leur avaient été consentis et les rapatriements de capitaux vers les pays donneurs sont déduits, afin d'obtenir le montant net des apports de fonds de la part de ces donneurs.

\section{Étapes de l'évaluation}

Les apports d'aide peuvent être mesurés soit au moment de la décision ferme (engagement), soit lorsque le transfert de fonds d'un pays à l'autre devient effectif (décaissement). L'évaluation peut porter sur les décaissements bruts - c'est-à-dire le 
montant total des transferts de capitaux vers le pays partenaire au cours d'une période donnée - ou nets - après déduction des remboursements de principal de prêts sur la même période. C'est généralement sur la base des versements nets d'APD que se fait l'évaluation de l'effort des donneurs en matière d'aide au développement.

\section{Méthodes de collecte de données}

Questionnaire du CAD - Les membres du CAD remplissent chaque année au mois de juillet, un ensemble de dix tableaux statistiques récapitulant le montant et la destination des aides qu'ils ont accordées au cours de l'année précédente. Les informations précises ainsi collectées concernent la destination, la forme, les conditions, le secteur et le degré de subordination des apports de fonds publics. Les organismes multilatéraux doivent compléter une version simplifiée de ce questionnaire. Les membres du CAD complètent en outre une fois par an, en avril, un questionnaire préliminaire d'une page sur les principaux agrégats du CAD.

Système de notification des pays créanciers (SNPC) - Ce système permet de notifier les transactions publiques effectuées à titre individuel (APD/AP ou autres apports de fonds publics) en faveur du développement. Les rapports sont transmis directement par les organismes officiels participants, notamment les agences bilatérales et multilatérales, les institutions de crédit au développement et les organismes de crédit à l'exportation. Tous les membres du CAD établissent des rapports dans le cadre de ce système, très complets pour 17 d'entre eux, partiels pour cinq autres. L'analyse de ces données sur les versements et la situation relative au remboursement des prêts permet au Secrétariat de l'OCDE de calculer le poids de la dette des pays en développement et en transition.

\section{Publications}

Les statistiques du CAD donnent lieu à trois publications principales :

- Rapport sur la coopération pour le développement - Ce rapport annuel, et plus particulièrement son annexe statistique, fournissent des informations détaillées sur les mouvements de fonds vers les pays en développement et en transition, notamment sur l'aide fournie par les membres du CAD.

- Répartition géographique des ressources financières allouées aux pays bénéficiaires de l'aide - Ce rapport annuel montre les apports de fonds dans les pays en développement et en transition, par origine et type de ressources.

- Rapports régionaux du système de notification des pays créanciers - Cette publication annuelle - en cinq volumes, par région - informe sur les notifications de projets/d'activités, par pays bénéficiaire et par secteur.

Ces différentes publications sont également disponibles sous forme électronique, sur un CD-ROM intitulé Statistiques sur le développement international. On pourra se procurer des informations complémentaires sur les statistiques $\mathrm{du} C A D$ à l'adresse Internet www.oecd.org/dac/stats. Par ailleurs, l'accès à toutes les bases de données est possible par abonnement au site Internet du CAD, à l'adresse www.ocde.org/dacocde/50/17/5037721.htm. 
Tableau A3.1. Liste des bénéficiaires de l'aide établie par le CAD - $\mathbf{1}^{\mathrm{er}}$ janvier 2004

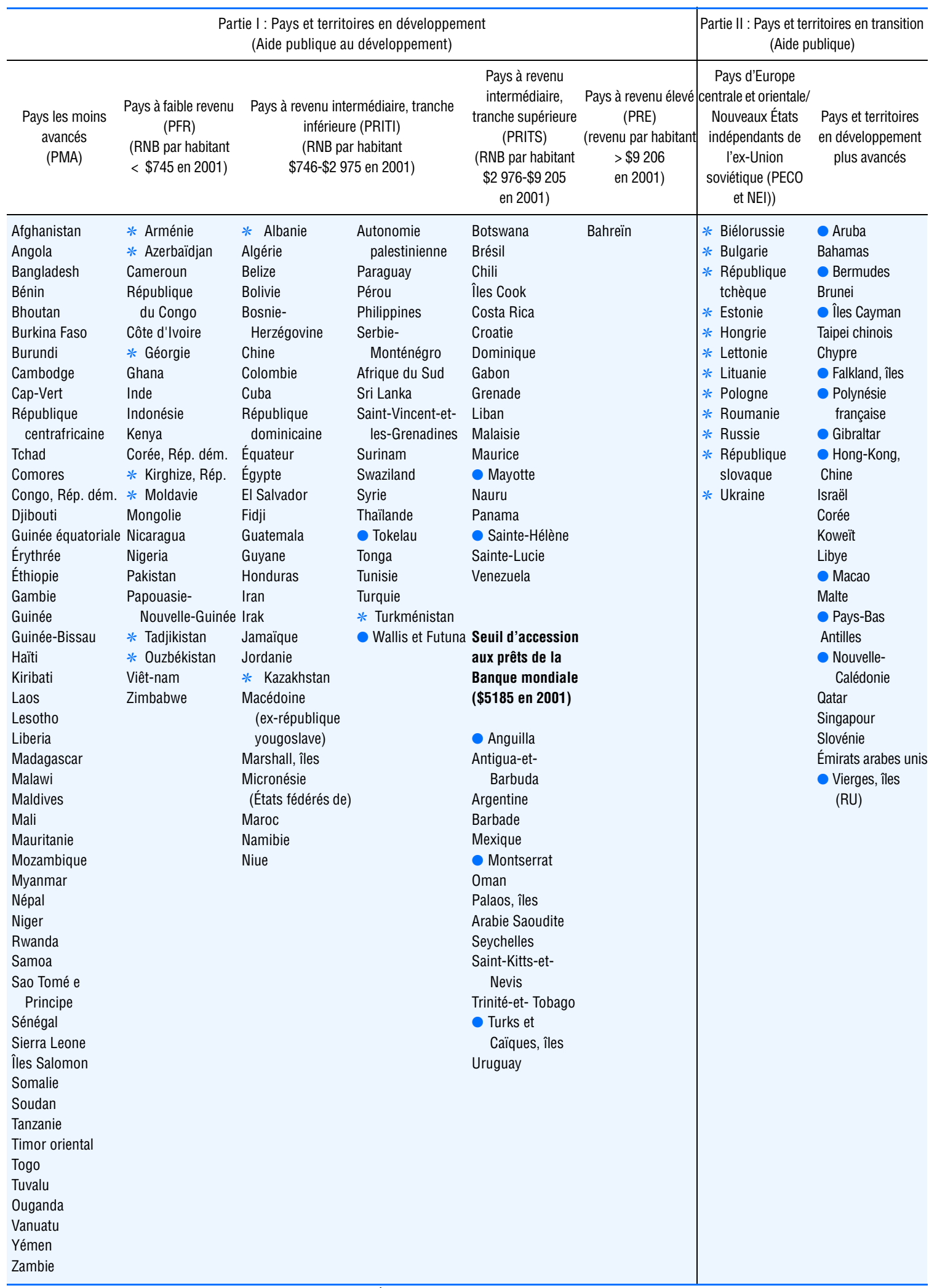

* Pays d'Europe centrale et orientale et Nouveaux États indépendants de l'ex-Union soviétique (PECO/NEI).

- Territoire.

Source : www.oecd.org/dataOCDE/50/17/5037721.htm. 


\section{Prise en compte de la réduction de la pauvretée et d'autres questions transversales: VIH/sida, égalité homme-femme et environnement}

Comme indiqué au chapitre 6, il importe, pour atteindre des objectifs globaux en matière de développement, de prendre en compte certains aspects de portée générale, comme la réduction de la pauvreté et l'égalité homme-femme : ces questions sont en effet déterminantes pour les résultats et l'impact d'un programme d'aide extérieure sous tous ses aspects, et ne peuvent dès lors faire l'objet ni d'actions indépendantes du contexte spécifique, ni d'une gestion sectorielle. Il s'agit au contraire d'introduire et d'intégrer de telles considérations transversales dans le programme d'ensemble de toute agence de développement. La présente annexe s'inspire de l'expérience de plusieurs pays membres du CAD, ainsi que de documents du CAD de l'OCDE; elle énonce les bonnes pratiques visant à l'intégration des questions transversales en général et elle analyse plus spécifiquement certaines d'entre elles, comme la réduction de la pauvreté, l'égalité entre les sexes, le VIH/sida et l'environnement.

L'intégration d'une question transversale sous-entend qu'on la connaît bien et qu'on la prend pleinement en considération dans les décisions et les processus analytiques, ainsi que pour l'élaboration des politiques et la planification. Cette définition de l'intégration montre que ce concept va bien au-delà de la simple prise en compte d'un problème transversal et qu'il exige d'importants investissements en temps et en ressources. Il n'est pas possible d'intégrer de nombreux aspects dans un programme de développement sans risquer de diluer l'importance de ces éléments quant aux résultats du programme. Il est plus réaliste de se concentrer sur une ou deux considérations transversales en ligne avec les objectifs d'ensemble et de les prendre intégralement en compte dans tous les aspects de ce programme. Deux ou trois autres questions transversales peuvent alors éventuellement y être intégrées, cette intégration dans les politiques et les procédures nécessitant alors, mais à un degré moindre, des ressources, des compétences et un engagement.

Les agences de développement ont accumulé une expérience considérable grâce à leur prise en compte de la problématique homme-femme. Les stratégies d'intégration diffèrent parfois selon le type de problème et le contexte (dans les pays partenaires), mais cette expérience fournit de précieux enseignements. La prise en compte de considérations transversales nécessite des stratégies d'intégration dans les politiques et dans les opérations de l'organisme ou du ministère en charge du développement, des stratégies 
d'intégration au-delà du cadre de l'organisme en cause - c'est-à-dire impliquant les organismes partenaires comme les organisations multilatérales et les ONG - et des stratégies à l'appui d'une prise en compte de ces questions au sein des pays partenaires en tant que partie du mode d'opération en partenariat.

Les stratégies d'intégration les plus importantes comportent l'élaboration d'une politique claire applicable à l'ensemble de l'agence de développement, un engagement de sa direction, la définition de stratégies d'intégration au niveau du pays, la mise en place d'unités spécialisées ou le recours à des experts, ainsi que l'acquisition de compétences analytiques adéquates. Afin d'atteindre les résultats recherchés, il sera parfois nécessaire d'adopter des stratégies spécifiques au problème en cause.

La présente annexe expose de façon assez détaillée des stratégies d'intégration du problème de la lutte contre la pauvreté, dont un grand nombre sont susceptibles de s'appliquer également à d'autres questions transversales. Dans les sections ci-après, on abordera des stratégies spécifiques au VIH/sida, à la sexospécificité et à l'environnement, qui pourront venir s'ajouter aux stratégies principales exposées pour la prise en compte de la lutte contre la pauvreté.

\section{Intégration de la question de la réduction de la pauvreté}

Comme indiqué dans les Objectifs du millénaire pour le développement (OMD), les membres du CAD se sont engagés à faire reculer la pauvreté, ce qui a incité la plupart d'entre eux à adopter officiellement la lutte contre la pauvreté comme objectif d'ensemble de leur coopération au développement. "Les lignes directrices du CAD », La réduction de la pauvreté soulignent bien que tout organisme désireux de s'attaquer efficacement à ce problème doit intégrer la réduction de la pauvreté dans l'ensemble de ses politiques et de ses opérations. Cela implique des adaptations dans les structures organiques, les incitatifs, les pratiques, les systèmes et jusque dans la culture propre à l'agence concernée.

\section{Stratégies d'intégration de la réduction de la pauvreté ${ }^{1}$}

Rôle de la direction de l'organisme d'aide, vision et engagement

- La hiérarchie doit faire preuve de détermination, tant au niveau politique qu'au niveau de la définition de l'action, de façon à mobiliser et focaliser la motivation et la participation de l'ensemble du personnel, des autres organes gouvernementaux et de la société civile autour d'une action plus résolument et plus fermement tournée vers le soutien à la lutte contre la pauvreté dans les pays partenaires.

- L'organisme d'aide doit se donner une ambition claire, un cadre politique et une stratégie pour s'attaquer à la pauvreté.

- La fixation d'objectifs multiples (développement durable, réduction de la pauvreté, égalité homme-femme, conflits ou objectifs nationaux de politique étrangère par exemple) est créatrice de tensions. Les dirigeants doivent clarifier ces objectifs en consultant largement le personnel, afin de déterminer les points de complémentarité, de faciliter les arbitrages et d'éviter des différends lors des débats ultérieurs.

- Il est essentiel de mettre les responsables des ressources humaines en relation avec le personnel chargé de définir les politiques: les dirigeants doivent comprendre les conséquences pratiques de la stratégie et de la politique quant à la gestion du personnel et au renouvellement des ressources humaines. 
- Les responsables de l'organisme, à tous les niveaux - et particulièrement au niveau des cadres intermédiaires - doivent proclamer haut et fort leur engagement au service de la lutte contre la pauvreté.

\section{Élaboration des politiques et des programmes}

- Il convient d'adapter le plus parfaitement possible les politiques à la stratégie du pays partenaire en matière de lutte contre la pauvreté, de les introduire dans les stratégies de l'agence au bénéfice des différents pays, et de prévoir des stratégies pour l'inclusion d'indicateurs de niveaux concernant le pays en cause.

- L'objectif de réduction de la pauvreté devrait imprégner l'ensemble des processus de planification à l'œuvre au sein de l'organisme d'aide (y compris les stratégies applicables aux différents pays, les approches sectorielles et les interventions au titre de projets). Les programmes et projets devraient faire l'objet d'une évaluation systématique axée sur leur contribution potentielle à la réduction de la pauvreté dans le cadre du système de sélection et des procédures d'approbation en vigueur dans l'organisme.

- Il convient d'envisager des ajouts ou des révisions au règlement du personnel, afin de mettre au centre des objectifs, des opérations et des performances de l'agence le rôle de cette dernière dans la lutte contre la pauvreté.

- Il importe également de promouvoir une culture institutionnelle qui contribue à la réduction de la pauvreté : en effet, une culture axée sur une simple aide financière ou sur les bonnes paroles peut diluer, voire contrecarrer, le dynamisme de l'agence en la matière. Il est préférable de mettre au point des approches, des instruments ou des stratégies permettant d'anticiper ou d'atténuer les difficultés rencontrées en fin d'exercice budgétaire pour effectuer les versements.

- Les liens doivent être renforcés entre les activités visant à favoriser l'intégration, au siège et sur le terrain.

- Les cahiers des charges définis pour des projets de recherche, des études ou l'élaboration de programmes doivent tenir compte des objectifs de réduction de la pauvreté. Des objectifs concrets et des normes en matière de réduction de la pauvreté doivent figurer dans les conventions et les accords de partenariat conclus avec des organismes extérieurs, des ONG ou des consultants. Les accords de partenariat devraient prévoir des dispositifs incitatifs et des sanctions, ainsi que des systèmes clairement définis de suivi des performances.

- Une interaction avec des partenaires multilatéraux est essentielle afin de s'assurer que ces derniers acceptent de promouvoir la lutte contre la pauvreté dans les grandes orientations et les activités de leurs propres organismes d'aide.

- Il importe de veiller à ce que l'importance des objectifs de réduction de la pauvreté soit intégrée dans les évaluations institutionnelles des partenaires multilatéraux.

\section{Mise en place de capacités organiques}

- Exploiter les compétences disponibles au sein de l'organisme d'aide, en aidant le personnel sectoriel à acquérir des compétences dans le domaine de la lutte contre la pauvreté et à les intégrer dans ses activités. 
- S'attacher à mettre en place de "nouvelles compétences " :

* Pour le partenariat : médiation, coordination, aptitude à établir des relations (écoute active, recherche de consensus, sens de la négociation, diplomatie).

* Pour le personnel diplomatique: aptitude à comprendre les problèmes de développement, à prendre des risques et à collaborer avec les partenaires sur le terrain.

* Pour l'ensemble du personnel de terrain : souplesse d'esprit, capacités d'adaptation et d'autocritique, aptitude à la réflexion latérale.

- La prise en considération des nombreuses dimensions de la pauvreté exige que soient constituées et déployées, à l'échelle nationale, des équipes pluridisciplinaires dûment formées et compétentes dans de nombreux domaines. Il importe de réunir des compétences d'ordre macro-, méso- et micro-économique.

\section{Politique du personnel}

- Le recrutement (personnel permanent, temporaire et diplomatique) doit privilégier les compétences et les performances en matière de lutte contre la pauvreté, ainsi que l'aptitude à travailler en équipe et l'expérience de fonctions de coordination (facilitation, sens de l'écoute et de la négociation).

- Il est nécessaire d'apporter un appui additionnel au personnel en poste dans les missions locales susceptible de manquer d'autorité et d'avoir besoin de soutien.

\section{Formation}

- Il convient de renforcer les liens entre les objectifs stratégiques de l'organisme, les programmes d'activités des unités et les «objectifs de résultats » des membres du personnel, afin d'accroître la cohérence interne et de consolider les efforts déployés.

- Les mandats des directeurs territoriaux et des chefs de programme doivent comporter des objectifs clairs en matière de réduction de la pauvreté et de partenariat, dont doivent tenir compte également les évaluations relatives à leurs propres performances et les critères d'appréciation de leur personnel.

- Pour être crédibles, les systèmes de gestion des performances doivent se fonder sur des critères objectifs. Lorsque la réduction de la pauvreté constitue l'un de ces critères, les systèmes d'évaluation des organismes d'aide peuvent nécessiter la mise au point de méthodes permettant d'évaluer l'impact des stratégies mises en œuvre pour lutter contre la pauvreté.

- Les systèmes d'incitations doivent être souples, faciles à mettre en œuvre et prévus dans les textes.

- Il est important de répertorier et de comprendre les mesures institutionnelles d'incitation et de dissuasion (explicites et implicites) pour évaluer les actions visant à accroître la cohérence entre les objectifs de réduction de la pauvreté de l'organisme et les performances du personnel.

\section{Structures organiques}

- Encourager le travail en équipe en favorisant le décloisonnement entre les secteurs, afin d'aborder plus efficacement le caractère pluridimensionnel de la pauvreté et de dépasser les approches strictement sectorielles ou définies en fonction des donneurs. 
- Mettre en place des structures et des mécanismes pour l'intégration de la lutte contre la pauvreté dans les activités d'aide au développement. On peut recourir à des "champions » de la cause pour populariser ce combat au sein de l'organisme d'aide, dispenser des conseils, améliorer la communication horizontale et verticale entre les niveaux hiérarchiques et promouvoir les bonnes pratiques. Les ressources et le pouvoir de décision doivent revenir à ceux qui, dans l'organisme, plaident en faveur de la lutte contre la pauvreté.

- Des structures plus simples, moins hiérarchisées, encouragent mieux le recours au travail en équipe et permettent d'apprécier les compétences pluridisciplinaires du personnel ; elles s'en remettent davantage à la circulation de l'information, de même qu'aux réseaux. Il n'en reste pas moins qu'un certain degré de hiérarchie est nécessaire pour assurer les fonctions de responsabilité, de contrôle de la qualité et de leadership.

- Il faut bien comprendre que, si les structures organiques sont très importantes, ce sont les méthodes de travail informelles - déterminantes quant à la manière dont les personnes travaillent ensemble au sein de groupes et collaborent d'une structure à l'autre - qui comptent le plus.

\section{Suivi}

- Mettre au point des systèmes de suivi qui permettent d'enregistrer les résultats au regard des objectifs de réduction de la pauvreté. Les systèmes comportant des balises peuvent s'avérer utiles.

- Concevoir des méthodologies pour évaluer l'impact en matière de réduction de la pauvreté.

\section{Intégration de la question du VIH/sida}

Plusieurs pays membres du CAD - notamment le Canada, le Danemark, l'Irlande et le Royaume-Uni - considèrent le VIH/sida comme un problème transversal majeur à intégrer dans leurs programmes de coopération au développement. Des membres comme l'Irlande ont adopté pour politique d'intégrer la question du VIH/sida dans l'ensemble de leur programme.

Les stratégies essentielles régissant l'intégration de la réduction de la pauvreté sont également d'application dans ce cas. Il s'agit notamment de mettre l'agence en situation de disposer d'un leadership, d'une vision et d'un engagement, de prendre en compte le $\mathrm{VIH} /$ sida dans l'élaboration des programmes, de doter l'agence de la capacité organisationnelle nécessaire à cette intégration, ainsi que de systèmes de suivi. En outre, certaines stratégies sont propres au cas du VIH/sida ou exigent une attention supplémentaire en raison de la nature particulière du problème.

\section{Stratégies d'intégration du VIH/sida ${ }^{2}$}

Élaboration des politiques et des programmes

- Veiller à ce que les déclarations de politique générale abordent le VIH/sida comme un problème de développement plutôt que comme un problème de santé.

- S'attacher, en coopération avec les pays partenaires et les partenaires clés dans le domaine du développement, à recueillir des informations sur l'état de la pandémie. 
- Envisager un soutien au renforcement des capacités de recherche afin de mieux appréhender l'étendue de l'épidémie et son cheminement.

- Déterminer si les partenaires sont prêts à aller de l'avant pour la mise en place de programmes de lutte contre le VIH/sida et réfléchir aux moyens d'orienter d'autres programmes de manière à prendre en compte indirectement le VIH/sida.

- Envisager la mise au point de stratégies anti-VIH/sida à l'échelle du pays.

- Susciter des occasions de dialogue au niveau national - il est essentiel d'obtenir la participation des directeurs des programmes destinés au pays, ainsi que du personnel diplomatique de haut rang.

- Dans le cadre de la mise au point du programme, envisager d'accorder un appui aux organisations non gouvernementales capables de travailler avec des groupes vulnérables et à haut risque.

- Veiller à ce que soient perçus clairement les liens avec les programmes concernant la réduction de la pauvreté et l'égalité homme-femme.

- Déterminer comment le VIH/sida peut être intégralement pris en compte par le biais de programmes sectoriels - il est indispensable de déborder du seul secteur de la santé.

- Examiner les études d'impact sur le VIH/sida de tout nouveau programme ou projet.

- Inclure, chaque fois que c'est possible, des personnes vivant avec le VIH/sida dans les processus d'élaboration et de mise en œuvre des programmes.

- Mettre en place des indicateurs adéquats et mesurables, et contrôler l'impact des programmes.

\section{Mise en place de capacités organiques}

- Elaborer des programmes de formation et de sensibilisation, indispensables à l'intégration du problème du VIH/sida en raison des idées fausses, des malentendus et des préjugés encore présents chez de nombreuses personnes quant aux causes, à la nature et à la diffusion de l'épidémie.

- Prévoir, au bénéfice du personnel clé, une formation spécifique lui permettant de se faire l'avocat de la cause et de favoriser le dialogue.

- Prodiguer une formation au personnel d'encadrement des missions locales, notamment au niveau des ambassadeurs : en effet, en dépit de la nature sensible de la question, ce personnel est bien placé pour la soulever et en discuter à un niveau élevé du gouvernement du pays partenaire.

- Assurer la formation et appuyer le développement des capacités en matière de prise en compte du VIH/sida chez les consultants et les partenaires de type ONG.

- Encourager l'acquisition des aptitudes analytiques nécessaires à l'évaluation de l'impact des programmes et des projets sur le VIH/sida.

- Définir des priorités quant aux aptitudes permettant de prendre en compte le VIH/sida en dehors du secteur de la santé.

- Mettre en place un programme VIH sur les lieux de travail, prévoyant notamment la formation à la sensibilisation pour tous les niveaux de personnel. À cet égard, les bonnes pratiques sur le lieu de travail comprennent notamment l'accès à l'information, des consultations et des dépistages confidentiels, la fourniture de préservatifs, ainsi que 
l'élaboration d'une politique de l'organisme d'aide sur l'accès au traitement pour le personnel infecté.

\section{Prise en compte de la question de l'égalité homme-femme}

La notion d'approche intégrée de l'égalité homme-femme est fréquemment abordée depuis la Conférence internationale des Nations unies sur les femmes, tenue en 1995, et l'adoption du Programme d'action de Pékin. Pour les Nations unies, l'intégration des questions de genre :

" consiste à évaluer les implications des femmes et des hommes dans toute action planifiée comprenant la législation, les procédures ou les programmes dans tous les domaines et à tous les niveaux. Cette stratégie permet d'intégrer les préoccupations et les expériences des femmes et des hommes à la conception, à la mise en œuvre, au contrôle et à l'évaluation des procédures et des programmes dans toutes les sphères politiques, économiques et sociétales pour qu'ils en bénéficient de manière égale et que l'inégalité actuelle ne soit pas perpétuée. " L'objectif ultime de l'intégration des questions de genre est l'égalité homme-femme. (Conseil économique et social des Nations unies, 1997.)

De nombreuses agences de développement s'attachent depuis un certain temps à promouvoir l'égalité entre les sexes ainsi qu'une autonomisation des femmes, et l'on note des progrès sensibles, tant dans les pays développés que dans les pays en développement, où les femmes jouissent d'une plus grande liberté et de davantage de pouvoir qu'auparavant. Pourtant, une étude récente montre qu'il subsiste des obstacles à l'égalité homme-femme : nombre de pays donneurs ne se sentent pas de responsabilité quant aux processus menant à l'égalité, ils s'abstiennent d'en faire des objectifs prioritaires dans leurs stratégies par pays et ne donnent pas de directives quant à la mise en œuvre d'une politique allant dans le sens d'une telle égalité3. Il convient de promouvoir sans relâche et de soutenir activement l'égalité homme-femme comme indiqué par l'adoption, au titre d'objectif du millénaire pour le développement, de l'égalité des sexes et de l'autonomisation des femmes.

Les stratégies d'égalité homme-femme doivent être formulées dans le cadre de modifications aux politiques et aux approches applicables au développement, de changements au sein même des pays partenaires et d'évolutions dans le contexte international. Ainsi, le recours plus fréquent à des instruments d'assistance tels que les approches sectorielles et les aides budgétaires directes, de même que la définition de stratégies nationales de réduction de la pauvreté ont des conséquences sur les stratégies de prise en compte.

Comme pour la pauvreté et le VIH/sida (voir sections précédentes), l'intégration de l'égalité homme-femme doit comporter des stratégies visant à développer le leadership de l'organisme d'aide, à assurer une vision et un engagement, à refléter l'objectif d'égalité entre les sexes dans la définition des programmes, à mettre en place une capacité organisationnelle permettant d'intégrer l'égalité homme-femme, et à élaborer des systèmes de suivi pour chaque sexe visant spécifiquement à mesurer l'impact des programmes sur les femmes et sur les relations entre hommes et femmes. Au niveau opérationnel, il pourra s'avérer nécessaire de sexospécifier certaines activités et d'introduire une discrimination positive lorsque l'un des deux sexes se trouve particulièrement désavantagé. 


\section{Stratégies pour la prise en compte de l'égalité homme-femme ${ }^{4}$}

Élaboration des politiques et des programmes

- Formuler des politiques et des stratégies exposant les buts à atteindre, ainsi que les moyens par lesquels on pourra évaluer les progrès d'ensemble en matière de soutien à l'objectif d'égalité homme-femme et d'autonomisation des femmes.

- Faire participer des experts en sexospécificité à tous les aspects de la définition des politiques.

- Dans le cadre d'un dialogue avec les parties prenantes sur les objectifs et les activités relatifs à l'égalité entre les sexes, parvenir à une vision commune de ces objectifs avec les pays partenaires, et notamment avec les instances gouvernementales, les associations non gouvernementales et la société civile.

- En se fondant sur la participation des pays partenaires et un haut degré d'appropriation par ces derniers des buts à atteindre, s'assurer que les stratégies nationales reflètent bien ces buts en matière d'égalité homme-femme et de renforcement du pouvoir des femmes.

- Les engagements à long terme vis-à-vis des partenaires et des activités liées au développement constituent un aspect important du modèle de partenariat en vue du développement et jouent également un rôle primordial pour que des progrès durables soient possibles dans le sens d'une égalité, tant au niveau d'ensemble qu'à celui des cellules individuelles.

- Veiller à ce que les documents relatifs aux projets comportent des actions visant à promouvoir une meilleure égalité en termes d'influence, d'opportunité et de bénéfice.

- Définir des créneaux stratégiques pour lancer la promotion de l'égalité entre les sexes.

- S'assurer que les objectifs d'égalité homme-femme sont pris en compte dans les descriptions d'emploi du personnel et dans l'évaluation des performances de ce dernier.

- Encourager la participation des femmes, tout autant que des hommes, à la conception et à la mise en œuvre des projets et des programmes.

- Évaluer l'impact de tous les projets et programmes sur l'égalité homme-femme.

- Procéder, pour les projets et programmes, à une analyse des parties prenantes axée sur la problématique homme-femme.

- Coopérer avec les gouvernements partenaires afin d'aboutir à une recherche analytique et à des données ventilées par sexe.

Mise en place de capacités organiques

- Examiner à intervalles réguliers l'aptitude du personnel à prendre en compte la question de l'égalité entre hommes et femmes.

- Mettre au point des programmes de formation pertinents au plan opérationnel.

- Prévoir des cours de mise à jour pour le personnel d'encadrement et les agents bénéficiant de contrats de longue durée.

- Élaborer de nouveaux programmes de formation tenant compte de changements intervenus dans le contexte du développement. Exemples: comment intégrer cette problématique dans le cadre d'approches sectorielles et d'aides budgétaires ; élaboration 
d'objectifs liés à l'égalité homme-femme pour les procédures concernant une gestion axée sur les résultats.

- Mettre en place, dès le début, une unité spécialisée dans la problématique hommefemme, ainsi que des points de contact, afin de disposer d'un soutien et de compétences dans les différents aspects de la prise en compte des questions d'égalité entre sexes.

- S'assurer qu'il existe dans le pays des personnes compétentes capables d'analyser les composantes sociales et sexoproblématiques et de prodiguer un soutien pour la mise en œuvre et le suivi de toutes les stratégies.

- Veiller à ce que les autres programmes de formation (par exemple ceux concernant la réduction de la pauvreté, le VIH/sida ou l'agriculture) tiennent compte de la question de l'égalité entre les hommes et les femmes.

\section{Mise en place de capacités dans les pays partenaires}

Dans le cadre du modèle de partenariat, les membres du CAD ont le souci de renforcer les capacités internes des pays partenaires, et notamment celles portant sur la prise en compte ou l'intégration dans les politiques et les pratiques d'importants problèmes d'ordre transversal. À cet égard, un des éléments essentiels concerne l'aptitude à identifier les besoins liés aux différences et aux disparités entre hommes et femmes, et à y remédier.

- Appuyer les stratégies visant à renforcer les capacités des pays partenaires à analyser les politiques, les programmes et les cultures institutionnelles et à introduire des stratégies de changement qui contribuent à l'égalité entre hommes et femmes.

- Aider les partenaires à identifier des stratégies propres à augmenter la représentation des femmes à l'échelon décisionnel et au niveau de définition des politiques.

- Appuyer les systèmes statistiques nationaux afin de faire progresser la collecte et la mise à disposition de données ventilées par sexe.

- Soutenir la mise en place de compétences en matière de recherche et de recherche opérationnelle portant sur l'égalité d'opportunité et de résultat.

\section{Suivi}

- Mettre au point des indicateurs tenant compte du sexe des individus et permettant d'évaluer l'impact des actions.

\section{Prise en compte de l'environnement et du développement durable}

La protection et une meilleure gestion de l'environnement représente une stratégie essentielle en vue de la réduction de la pauvreté. Certains pays membres du CAD consentent une assistance extérieure importante au bénéfice de projets liés à l'environnement; d'autres pays membres s'intéressent à des domaines tels que l'agriculture et l'énergie, qui comportent des implications écologiques importantes; plusieurs pays membres s'efforcent de traiter les problèmes écologiques comme une considération transversale, que le programme en cause aborde directement ou non les préoccupations environnementales.

Deux aspects principaux distinguent des autres sujets la prise en compte de l'environnement. En premier lieu, quelle que soit la portée de la composante écologique du programme d'ensemble, il est obligatoire de soumettre tous les projets et programmes à 
l'Étude d'impact sur l'environnement (EIE) (Recommandation du Conseil de l'OCDE concernant l'évaluation environnementale des projets et programmes d'aide au développement, 1985). Tous les membres sont donc tenus de disposer de certaines capacités en matière d'EIE. Deuxièmement, la plupart des membres du CAD sont également signataires d'accords multilatéraux sur les importantes questions écologiques mondiales. Cela les engage à aider les pays partenaires, par le biais de programmes d'assistance extérieure, à gérer les préoccupations de ces pays en matière d'environnement.

À part ces différences, les grands principes de la prise en compte restent identiques, même si, au niveau des approches, celles concernant l'environnement sont très spécifiques. Les stratégies clés exposées pour la prise en compte de la réduction de la pauvreté, du VIH/sida et de la sexospécificité restent valables. Il s'agit notamment de stratégies visant à développer le leadership de l'organisme d'aide, à assurer une vision et un engagement, à refléter les objectifs de développement durable dans la définition du programme, à mettre en place une capacité organisationnelle permettant d'intégrer le développement durable et à élaborer des systèmes de suivi adéquats. Néanmoins, il convient que tous les membres du CAD s'attachent en outre à répondre aux exigences de l'EIE et à aider les pays partenaires à propos des problèmes écologiques de portée mondiale.

\section{Stratégies pour la prise en compte des questions d'environnement et de développement durable ${ }^{5}$}

Étude d'impact sur l'environnement (EIE) (" désigne les exigences obligatoires)

- *Établir une unité indépendante pour l'EIE.

- Élaborer des directives concernant l'EIE qui soient conformes avec les principes existants et les lignes directrices du CAD (voir les Lignes directrices sur l'aide et l'environnement (1992-1995): $N^{\circ} 1$ : Bonnes pratiques pour les études de l'impact sur l'environnement exercé par les projets de développement.

- Promouvoir et renforcer, chez tout le personnel en charge du développement, la compréhension des exigences EIE.

- *La soumission à l'EIE est impérative pour tous les projets. L'évaluation est à effectuer soit par l'unité EIE (et non par des spécialistes de l'environnement ayant contribué à la conception du projet), soit par un organisme spécialisé agissant en sous-traitance pour l'unité EIE.

- Garantir que des capacités adéquates sont disponibles pour l'EIE.

- Le nombre de membres de l'unité EIE et les compétences qu'ils réunissent doivent être en proportion avec la nature et le volume des travaux comportant des implications environnementales significatives.

- Le personnel EIE devrait être associé au processus de définition des politiques.

- L'unité EIE devrait représenter une source d'information et d'assistance pour le personnel de l'agence.

- Appuyer les initiatives visant à renforcer les capacités des pays partenaires en ce qui concerne l'EIE. 
Stratégie environnementale au niveau des programmes

Élaboration des politiques et des programmes

- Veiller à ce que les politiques comprennent des objectifs liés aux préoccupations d'environnement durable.

- Associer des spécialistes de l'environnement à la définition des politiques.

- S'assurer que les préoccupations environnementales figurent dans les documents relatifs aux stratégies applicables au pays en cause et dans les évaluations de la pauvreté dans ce pays.

- Recourir à des évaluations environnementales stratégiques (EES) à l'appui de l'élaboration des politiques et déterminer les domaines dans lesquels une aide pourrait être apportée afin d'inverser des tendances négatives et d'en réduire l'impact sur les populations pauvres.

- Dialoguer avec le pays partenaire et les partenaires du développement afin de s'assurer que, dans tous les secteurs, les politiques clés comme la stratégie de réduction de la pauvreté et les approches sectorielles prennent en considération les contraintes environnementales et n'ignorent pas les opportunités de bénéfices pour l'environnement.

- Identifier les occasions de coopérer avec le secteur privé afin d'améliorer les normes environnementales (par exemple: encourager une production moins polluante de la part de l'industrie).

- Améliorer la préparation aux catastrophes et introduire des clauses de prévention et de limitation des catastrophes écologiques dans les stratégies nationales de développement durable et dans les projets de développement à long terme (voir OCDE/DAC (1994), Lignes directrices sur l'aide et l'environnement: $N^{\circ} 7$ : Lignes directrices à l'intention des organismes d'aide visant à limiter les conséquences des catastrophes).

- Lancer des études de cas sur les opportunités saisies par l'agence en matière de réduction de la pauvreté et de protection de l'environnement, et diffuser ces études à d'autres agences et aux principaux partenaires.

- Elaborer des stratégies intersectorielles visant à promouvoir le développement durable (voir OCDE/CAD (2001), « Lignes directrices du CAD », Stratégies de développement durable.)

- Veiller à ce que les politiques et les programmes comportent des passerelles entre protection durable de l'environnement, réduction de la pauvreté, VIH/sida et égalité homme-femme.

- S'attacher, en concertation avec les principaux partenaires multilatéraux, à introduire des objectifs et indicateurs de durabilité.

Mise en place de capacités opérationnelles

- Créer une unité spécialisée en matière d'analyse environnementale stratégique ou affecter du personnel compétent dans ce domaine à des équipes clés au sein de l'agence.

- Veiller à ce que le siège social et le personnel de terrain puissent accéder à du personnel spécialisé compétent dans ce domaine.

- Élaborer des lignes directrices concernant l'analyse environnementale stratégique.

- Doter le personnel d'aptitudes à appréhender les dimensions environnementales des politiques et des programmes. 
- Acquérir des capacités analytiques relatives aux relations entre la dégradation de l'environnement et la pauvreté.

- Veiller à une formation adéquate du personnel quant aux implications écologiques du développement durable.

Suivi

- Mettre au point des systèmes permettant de contrôler l'impact sur l'environnement des projets et programmes pendant leur phase de mise en œuvre.

Renforcement des capacités au sein des pays partenaires

- Encourager l'introduction d'aspects relatifs à l'environnement et à la durabilité dans les stratégies nationales de réduction de la pauvreté.

- Appuyer les initiatives visant à développer des capacités en matière d'analyse environnementale stratégique.

\section{Préoccupations écologiques à l'échelle mondiale}

- Appuyer les initiatives visant à développer, au sein des pays partenaires, des capacités propres à permettre à ces pays de remplir leurs obligations au titre d'accords multilatéraux sur l'environnement et d'en tirer les bénéfices (Voir CAD de l'OCDE [1992], Lignes directrices du CAD sur l'environnement et l'aide, $n^{\circ} 4$, Lignes directrices sur les problèmes de dimension globale destinées aux organismes d'aide).

\section{Notes}

1. La présente section s'inspire de différentes sources, parmi lesquelles, "Les lignes directrices du CAD ", La réduction de la pauvreté, OCDE (2001), Paris.

2. La présente section s'inspire de plusieurs sources, parmi lesquelles : OCDE/Organisation mondiale de la santé (2003), "Lignes directrices et ouvrages de référence du CAD », Pauureté et santé, OCDE, Paris, et OCDE (2003), DAC Peer Review of Ireland (Examen de l'Irlande par les pairs), OCDE, Paris.

3. Hunt, J. (2004), Effective Strategies for Promoting Gender Equality, document élaboré pour le GENDERNET du CAD de l'OCDE.

4. La présente section s'inspire de plusieurs sources, parmi lesquelles : Derbyshire, H. (2002), Gender Manual: A Practical Guide for Development Policy Makers and Practitioners, DFID, London ; Hunt, J. (2004), Effective Strategies for Promoting Gender Equality, document établi pour le GENDERNET du CAD de l'OCDE ; OCDE (1999), Lignes directrices du CAD pour l'égalité homme-femme et le renforcement du pouvoir des femmes, Paris ; OCDE (n.d.), Gender Equality Tip Sheets, Paris.

5. La présente section s'inspire de plusieurs sources, parmi lesquelles : OCDE (1992-995), «Les lignes directrices sur l'environnement et l'aide ", Paris ; OCDE (2001), "Les lignes directrices du CAD » Stratégies de développement durable, Paris; La politique de l'ACDI en matière de développement durable (ACDI), disponible à l'adresse www.acdi-cida.gc.ca/cida_ind.nsf/0/8d822748c6f30b31852565450065e876/\$FILE/ ENV-nophotos-E.pdf et DFID (2000), Achieving Sustainability : Poverty Elimination and the Environment, DFID, London. 


\section{ANNEXE A.5}

\section{Gérer des projets et des programmes de développement ${ }^{1}$}

La gestion d'un cycle de projet (Project Cycle Management-PCM) commence à la conception initiale d'un projet ou d'un programme et s'achève avec l'évaluation qui suit la mise en œuvre. Ce processus met fortement l'accent sur les bénéficiaires potentiels, sur une évaluation approfondie et sur l'application d'un cadre logique. De nombreux organismes axés sur le développement ont élaboré ou adapté des méthodes de gestion d'un cycle de projet ou du cadre logique dans le contexte des programmes qu'ils mettent en œuvre.

La PCM et le cadre logique sont censés fournir une approche raisonnée et structurée en vue de la définition des priorités, des activités et des résultats escomptés pour une intervention donnée. Ces deux approches aident les responsables de la planification, de la mise en œuvre et de l'évaluation d'un projet à se concentrer sur les éléments d'une intervention qui sont jugés les mieux à même d'assurer le succès de cette dernière. La PCM peut s'appliquer à des projets et à des programmes tels que les approches sectorielles, ainsi qu'à des interventions à l'échelle locale ou nationale. Elle doit concerner aussi bien les activités de développement à gestion directe que celles qui sont confiées à des organismes de mise en œuvre extérieurs, à des cabinets de consultants ou à des ONG.

Un cycle de projet est un modèle détaillé portant sur le cycle de vie complet d'une intervention dans le domaine du développement, depuis la formulation du problème et la définition de l'intervention, jusqu'à l'évaluation et aux enseignements tirés. Il s'agit d'un processus continu dont chaque étape fournit les bases de la suivante. La subdivision d'une intervention en étapes distinctes contribue à faire en sorte que les décisions reposent sur des informations suffisantes et pertinentes, et met en lumière la nécessité de décisions sur l'opportunité de poursuivre dans la même voie ou de modifier les plans.

Un cycle de projet comprend un certain nombre d'étapes identifiables, dont la description varie d'un pays du CAD à l'autre, mais dont les plus importantes sont la définition, la conception, l'évaluation préliminaire, la préparation et l'approbation, la mise en œuvre et le suivi, et l'évaluation finale.

\section{Définition : développement de l'idée initiale et conception préliminaire}

La définition initiale d'un problème ou d'une question de développement, de même que l'idée d'une action ou d'une assistance financière de la part d'un membre du CAD, sont de plus en plus fréquemment le fait des gouvernements des pays partenaires. Il n'en reste pas moins que les autorités régionales ou locales, les groupes de la société civile, les 
collectivités, les organisations internationales ou les agences de développement peuvent mettre en évidence un problème et constater la nécessité d'une aide extérieure.

Quelle que soit l'origine de l'idée initiale, il importe que la conception préliminaire s'effectue de manière participative et comprenne une première analyse établie par les intervenants, afin d'établir qui sont les parties prenantes principales et secondaires, les bénéficiaires et les groupes clés qui conduiront l'action. Dans certains pays membres, une note définissant le concept initial et le budget préliminaire doit être approuvée avant la conception proprement dite du projet.

\section{Préparation/conception : plus approfondie, la phase de conception aborde les aspects techniques et opérationnels}

Au cours de la deuxième étape, la conception du projet se précise, définit les objectifs clés, les bénéficiaires, les activités à mener et les principaux risques, et aborde les aspects opérationnels. Dans leur majorité, les membres du CAD utilisent un cadre logique ou cadre d'intervention comme base de la préparation et de la conception du projet, puis du suivi et de l'évaluation.

\section{Cadre logique}

Le cadre logique, ou cadre d'intervention, est un outil de planification permettant d'établir la structure de base d'une intervention. Il fournit une méthode systématique pour déterminer et analyser les objectifs d'une action axée sur le développement et des hypothèses qui la sous-tendent. Bien que le cadre d'intervention constitue un outil essentiel pour la gestion d'un cycle de projet ou de programme, il ne représente qu'un aspect de ce cycle.

Le cadre logique cerne le but ou l'objectif global de développement que l'action envisagée contribuera à atteindre, la raison d'être de cette action - qui est l'objectif spécifique à atteindre pendant le cycle de vie du projet - les résultats escomptés, les différents types d'activités nécessaires pour obtenir ces résultats, les indicateurs à utiliser pour suivre le processus de réalisation de l'objectif et des buts, les résultats et les activités, et les moyens de vérification. L'inclusion d'une colonne relative aux hypothèses de base est indispensable, et définit les conditions favorables qui doivent être réunies dans l'environnement extérieur pour que l'objectif général, les buts et les résultats puissent être atteints.

La « logique " du cadre d'intervention s'articule de bas en haut, et de gauche à droite. En d'autres termes, les activités doivent conduire à des résultats, qui eux-mêmes permettent d'atteindre les buts, ce qui contribue à la réalisation de l'objectif global. Il faut cependant que les liens entre la structure du projet et l'environnement extérieur soient pris en compte et que les hypothèses se vérifient. Par conséquent, ainsi que l'illustrent les flèches du schéma ci-dessous :

- Si les activités sont menées à bien et que les hypothèses se vérifient, les résultats escomptés seront obtenus.

- Si les résultats escomptés sont obtenus et que les hypothèses se vérifient, les objectifs intermédiaires seront atteints.

- Si les objectifs intermédiaires sont atteints et que les hypothèses se vérifient, l'objectif global sera atteint également. 
Cette "logique" ainsi que la connexion entre la structure du projet et les circonstances extérieures ou les hypothèses apparaissent plus clairement dans l'exemple ci-après :

- Si l'activité 1.1 est menée à bien - en l'occurrence: constitution du groupe - et si l'hypothèse selon laquelle les membres des familles soutiennent les efforts des femmes pour acquérir de nouvelles compétences se vérifie, le résultat visé $n^{\circ} 1$ sera atteint et le groupe sera opérationnel.

- Si le résultat $n^{\circ} 1$ est atteint - le groupe est opérationnel - et si les vêtements produits sont effectivement de bonne qualité (hypothèse), l'objectif intermédiaire - création d'activités génératrices de revenus pour les femmes - sera atteint également.

\section{Tableau A5.1. Structure du cadre logique}

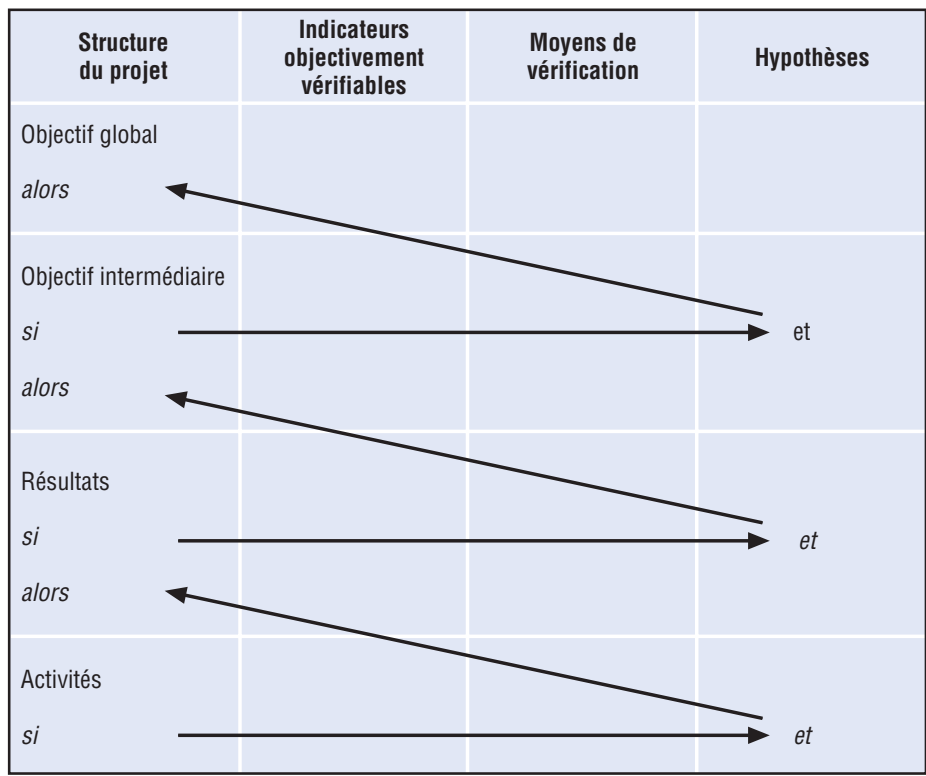

Seules sont prises en compte dans le cadre logique les hypothèses qui sont essentielles au succès du projet mais ne nécessiteraient pas la refonte ou l'annulation de l'action envisagée si elles ne se vérifiaient pas. Certaines activités peuvent être incluses dans la conception afin d'anticiper et de dépasser une hypothèse critique. Dans l'exemple ci-dessus, des activités visant à sensibiliser les familles à l'objectif visé et aux bénéfices que le projet représente pour elles à long terme pourraient accroître les chances de voir l'hypothèse se vérifier (soutien des membres des familles aux femmes qui s'efforcent de créer des activités génératrices de revenus).

La définition des indicateurs clés et des moyens de vérification est également très importante pour la mise en place du cadre logique et la conception de l'opération d'assistance. Les indicateurs peuvent être déterminés en coopération avec les principaux groupes intéressés et modifiés au cours du cycle de projet; des indicateurs supplémentaires peuvent également venir compléter les premiers. Il est généralement nécessaire de disposer à la fois d'indicateurs quantitatifs et qualitatifs, et plusieurs indicateurs peuvent s'appliquer à chaque objectif. Il convient également d'établir comment les indicateurs doivent être dissociés - par sexe, par niveau de revenu, par 
groupe cible, etc. - de manière à permettre un suivi précis de l'impact du projet sur différents groupes.

Les moyens de vérification fournissent une possibilité de contrôle croisé quant au choix et à la possibilité de mettre en œuvre des indicateurs. Lorsque cela est possible, indicateurs et moyens de vérification doivent s'appuyer sur des données d'origine nationale. En cas de moyens limités, il peut s'avérer nécessaire, dans le cadre de l'opération d'aide, de faciliter des études de base et des analyses continues, de même que le renforcement des capacités.

L'approche fondée sur le cadre logique est en outre pertinente pour les programmes complexes, comme ceux qui s'appliquent à l'échelle d'un secteur ou d'un pays. Dans de tels cas, un cadre logique principal peut se subdiviser en plusieurs cadres, l'objectif intermédiaire énoncé dans le premier cadre étant le même que l'objectif global de développement d'un ou plusieurs sous-éléments.

\section{3. Évaluation préliminaire : analyse du projet selon différentes perspectives :} économiques, sociales, institutionnelles et environnementales, impact sur la pauvreté, égalité homme-femme, lutte contre le VIH/sida

L'évaluation préliminaire est essentielle à la conception et à la mise en œuvre de toute action d'aide au développement, qu'il s'agisse d'un projet ou d'un programme. Cette première évaluation doit déterminer :

- La compatibilité avec les politiques gouvernementales.

- La compatibilité avec les actions des autres donneurs.

- La viabilité de l'opération.

- La pertinence de la conception technique.

- L'impact immédiat et les retombées sur les principaux groupes concernés, en particulier les personnes pauvres et vulnérables.

- L'impact probable sur les populations en fonction du sexe.

- Le degré de participation des groupes intéressés.

- La probabilité que les activités soient menées à bien et les résultats atteints conformément au calendrier prévu.

- La viabilité financière de l'opération.

- La situation relative aux institutions concernées.

- La nécessité d'activités permettant le renforcement des capacités institutionnelles.

- Les conséquences éventuelles sur l'environnement.

- Les risques susceptibles d'entraver la réalisation de l'objectif.

- Le caractère durable des conséquences bénéfiques, au-delà du cycle de vie de l'intervention.

\section{Préparation, approbation et financement : mise au point de la proposition, et mesures visant à répondre aux critères d'approbation et de financement}

La proposition finale doit tenir compte des critères de présentation et de contenu de chaque organisme de développement, ainsi que des cycles et des plafonds budgétaires prévus pour un programme particulier dans un pays donné. 


\section{Tableau A5.2. Exemple de cadre logistique - Création d'AGR pour les femmes}

\begin{tabular}{|c|c|c|c|}
\hline Structure du projet & Indicateurs objectivement vérifiables & Moyens de vérification & Hypothèses \\
\hline $\begin{array}{l}\text { Objectif de développement : } \\
\text { - Amélioration des conditions de vie } \\
\text { dans le village de pêcheurs. }\end{array}$ & $\begin{array}{l}\text { D'ici } 2005 \text { : } \\
\text { - } 70 \% \text { des maisons en briques. } \\
\text { - } 40 \% \text { des maisons ont un toit en tôle. } \\
\text { - } 90 \% \text { de diminution des cas de } \\
\text { maladies les plus répandues. } \\
\text { - } 90 \% \text { de la population peut payer les } \\
\text { impôts locaux. }\end{array}$ & Rapports de la communauté. & \\
\hline $\begin{array}{l}\text { Objectif intermédiaire : } \\
\text { - Création d'activités génératrices de } \\
\text { revenus pour les femmes. }\end{array}$ & $\begin{array}{l}\text { Proportion du revenu des ménages } \\
\text { générée par les activités de confection : } \\
\text { - } 5 \% \text { la } 1^{\mathrm{re}} \text { année. } \\
\text { - } 15 \% \text { la } 2^{\mathrm{e}} \text { année. } \\
\text { - } 30 \% \text { la } 3^{\mathrm{e}} \text { année. } \\
\text { - } \text { Au moins } 30 \% \text { la } 4^{\mathrm{e}} \text { année et les } \\
\text { suivantes. }\end{array}$ & Rapports de la communauté. & $\begin{array}{l}\text { - Les femmes contrôlent l'utilisation de } \\
\text { leurs revenus. } \\
\text { - L'assainissement et l'hygiène sont } \\
\text { améliorés. } \\
\text { - Les techniques de pêche sont plus } \\
\text { performantes. } \\
\text { - La pêche est pratiquée de façon } \\
\text { durable. } \\
\text { - Les pêcheurs cessent de migrer vers } \\
\text { d'autres îles. }\end{array}$ \\
\hline $\begin{array}{l}\text { Résultats : } \\
\text { 1. Groupe de couture opérationnel } \\
\text { 2. Les machines à coudre } \\
\text { appartiennent au groupe. }\end{array}$ & $\begin{array}{l}\text { 1. En moins de deux ans, le groupe } \\
\text { commercialise sa production. } \\
\text { 2. Le prêt pour les machines à coudre } \\
\text { est entièrement remboursé après } \\
18 \text { mois. }\end{array}$ & $\begin{array}{l}\text { - Rapports du groupe } \\
\text { de couture. } \\
\text { - Rapports financiers. }\end{array}$ & $\begin{array}{l}\text { - Les femmes formées restent sur l'île. } \\
\text { - Le groupe travaille } \\
\text { harmonieusement. } \\
\text { - Les vêtements produits sont de } \\
\text { bonne qualité. } \\
\text { - Le contrat pour les uniformes } \\
\text { scolaires est renouvelé. } \\
\text { - Les taxes d'importation de vêtements } \\
\text { restent élevées. }\end{array}$ \\
\hline $\begin{array}{l}\text { Activités: } \\
\text { 1.1 Constituer un groupe chargé } \\
\text { de la commercialisation des } \\
\text { vêtements. } \\
\text { 1.2 Former les femmes au métier } \\
\text { de la confection. } \\
\text { 2.1 Former les membres du groupe } \\
\text { au remboursement de leur crédit. } \\
\text { 2.2 Acheter les machines à coudre. }\end{array}$ & $\begin{array}{l}\text { 1. Groupe de couture } \\
\text { - Le groupe de couture est constitué } \\
\text { en deux mois. } \\
\text { - Les membres du comité directeur } \\
\text { sont nommés en quatre mois. } \\
\text { - } 35 \text { femmes appartenant à des } \\
\text { familles de pêcheurs deviennent } \\
\text { des couturières expérimentées. } \\
\text { - Elles se réunissent trois fois par } \\
\text { semaine pendant } 12 \text { semaines. } \\
\text { 2.1 Tous les membres du groupe } \\
\text { participent aux } 10 \text { séances (sur une } \\
\text { période de trois semaines). } \\
\text { 2.2 Au total, } 12 \text { machines ont été } \\
\text { achetées en trois mois. }\end{array}$ & $\begin{array}{l}\text { Rapports du groupe de couture. } \\
\text { Rapports sur la formation. } \\
\text { Rapports sur la formation. } \\
\text { Rapports financiers. }\end{array}$ & $\begin{array}{l}\text { - Les femmes suivent assidument } \\
\text { la formation. } \\
\text { - Les membres des familles } \\
\text { soutiennent le projet. } \\
\text { - Les machines à coudre restent en } \\
\text { bon état. } \\
\text { - Le contrat des uniformes scolaires } \\
\text { est assuré } \\
\text { - Le matériel de couture est disponible. } \\
\text { - Les paiements sont versés au groupe } \\
\text { aux dates prévues. }\end{array}$ \\
\hline
\end{tabular}

Source : Project Cycle Management Technical Guide, Programme d'analyse socio-économique selon le genre, FAO 2001, disponible à l'adresse : www.fao.org/sd/seaga/downloads/en/projecten.pdf.

\section{Mise en œuvre et suivi : mise en œuvre des activités et suivi permanent}

Partie intégrante du cycle de projet, le suivi est défini par le CAD de l'OCDE comme un "processus continu de collecte systématique d'informations, selon des indicateurs choisis, pour fournir aux gestionnaires et aux parties prenantes d'une action de développement en cours, des éléments sur les progrès réalisés, les objectifs atteints et l'utilisation des fonds alloués ${ }^{2} »$.

Le suivi permet d'examiner les progrès accomplis et de proposer les mesures nécessaires à la réalisation des objectifs. Cette responsabilité incombe à la fois aux organismes d'exécution et de financement du projet, mais il importe également de tenir compte des points de vue des parties prenantes et des bénéficiaires. Le cadre logique fournit la base de ce suivi, grâce aux indicateurs et aux moyens de vérification définis. Les 
indicateurs mesurent non seulement l'exécution d'activités bien spécifiées, mais permettent de s'assurer que les résultats escomptés pour le projet ont bien été obtenus et de connaître le degré de réalisation de l'objectif intermédiaire à chaque stade de la mise en œuvre. Dans certains cas, il peut s'avérer nécessaire de recueillir des données de base pendant la phase initiale du projet, afin que le suivi puisse s'effectuer à partir d'une base de référence suffisante. Il convient également d'enregistrer tout changement dans l'environnement extérieur et dans les principales hypothèses.

\section{6. Évaluation : lorsque le projet touche à sa fin, dès qu'il s'achève ou après son achèvement}

Selon le CAD de l'OCDE, une évaluation est "l'appréciation, aussi systématique et objective que possible, d'un programme ou d'une politique, en cours ou terminé, de sa conception, de sa mise en œuvre et de ses résultats. Le but est de déterminer la pertinence et l'accomplissement des objectifs, l'efficience en matière de développement, l'efficacité, l'impact et la durabilité. Une évaluation devrait fournir des informations crédibles et utiles permettant d'intégrer les leçons de l'expérience dans le processus de décision des bénéficiaires et des bailleurs de fonds ${ }^{3}$."

Les objectifs principaux de ce processus consistent donc à fournir une base objective à l'évaluation des performances de l'action de développement, à améliorer les résultats futurs grâce aux leçons de l'expérience, et à établir des grilles de responsabilités. Pour que ces objectifs puissent être atteints, l'évaluation doit être considérée comme une source d'enseignements au sein de l'organisation concernée, et, le cas échéant, contribuer à faire évoluer les comportements organisationnels.

Parmi les principaux aspects à aborder au cours d'une évaluation, figurent :

- La pertinence de l'intervention en fonction du contexte.

- Les répercussions - prévues et imprévues - de cette action et de toute contribution à la réalisation de l'objectif global de développement.

- La capacité de cette action à atteindre l'objectif fixé, et la mesure dans laquelle la réalisation de l'objectif peut être attribuée à l'intervention.

- L'efficacité de l'action, en fonction des intrants utilisés par rapport aux résultats obtenus.

- La pérennité des bénéfices une fois que l'assistance extérieure a pris fin.

\section{Notes}

1. La présente annexe s'inspire de plusieurs sources, parmi lesquelles: AusAID (1998), Review of the Evaluation Capacities of Multilateral Organisations, AusAID, Canberra ; Dearden, P. (2001), Programme and Project Cycle Management: Lessons from DFID and Other Organisations, Presentation to the Foundation for Advanced Studies for International Development; European Commission (2004), Aid Delivery Methods Volume 1: Project Cycle Management Guidelines, disponible à l'adresse : http://europa.eu.int/comm/europeaid/ qsm/documents/pcm_manual_2004_en.pdf; Guide technique - Gestion du cycle de projet, FAO/ASEG, disponible à l'adresse :www.fao.org/sd/seaga/downloads/en/projecten.pdf : Guidelines for Programme Design, Monitoring and Évaluation, ministère finlandais des Affaires étrangères (1998), Helsinki ; Introductory Notes about Project Cycle Management, PNUD (n.d.), disponible à l'adresse: www.undp.sk/uploads/ IntroductoryNotesaboutProjectCycleManagement.pdf.

2. OCDE (2002), Glossaire des principaux termes relatifs à l'évaluation et à la gestion axée sur les résultats, Paris.

3. Op. cit. 


\section{Bibliographie}

Acharya, de Lima et M. Moore (2004), Aid proliferation: How responsible are the donors?, Institute of Development Studies Working Paper 214, Brighton.

Addison, T., McGillivray, M. et M. Odedokum (2003), Donor Funding of Multilateral Agencies, Discussion Paper n 2003/17, UNU/WIDER.

Cox, A., Healey, J. et A. Koning (1997), How European Aid Works: A comparison of Management Systems and Effectiveness, Overseas Development Institute, The Chameleon Press Ltd., Londres.

Development Strategies (2003), Final Report: The Consequences of Enlargement for Development Policy, volume 1, IDC, Belgique.

DFID (2002), Working in Partnership with the World Health Organization, DFID, Londres.

Nohria, N. (1991), Note on Organisation Structure, Harvard Business School, Cambridge, Massachusetts.

Heimans, J. (2002), Multisectoral Global Funds as Instruments for Financing Spending on Global Priorities, Document d'orientation $n^{\circ} 24$, Département des affaires économiques et sociales des Nations unies.

Jerve, A. M. et H. Selbervik (2003), MOPAN: Report from the 2003 Pilot Exercise, Chr, Michelsen Institute, Bergen. Lancaster, C. (1999), Aid to Africa: So much to do, so little done, University of Chicago Press.

McGillivray, M. (2003), Aid Effectiveness and Selectivity: Integrating Multiple Objectives into Aid Allocations, Institut mondial de recherche sur l'économie du développement, Document d'orientation n² 2003/71, Helsinki.

OCDE (1992), Manuel de l'aide au développement : Principes du CAD pour une aide efficace, Paris.

OCDE Lignes directrices sur l'aide et l'environnement:

(1992), $n^{\circ} 1$. Bonnes pratiques pour les études de l'impact sur l'environnement exercé par les projets de développement, OCDE, Paris.

(1992), n² 2. Bonnes pratiques pour les études et stratégies nationales concernant l'environnement, OCDE, Paris.

(1992), $\mathrm{n}^{\circ}$ 3. Lignes directrices établies à l'intention des organismes d'aide concernant le déplacement involontaire et la réinstallation dans le cadre de projets de développement, $\mathrm{OCDE}$, Paris.

(1992), $\mathrm{n}^{\circ}$ 4. Lignes directrices sur les problèmes d'environnement de dimension globale destinées aux organismes d'aide, OCDE, Paris.

(1992), $n^{\circ}$ 5. Lignes directrices établies à l'intention des organismes d'aide relatives à la gestion des produits chimiques, OCDE, Paris.

(1994), $n^{\circ} 6$. Lignes directrices établies à l'intention des organismes d'aide pour la lutte contre les parasites et la gestion des pesticides, OCDE, Paris.

(1994), $n^{\circ}$ 7. Lignes directrices établies à l'intention des organismes d'aide relatives à la réduction de l'incidence des catastrophes naturelles, OCDE, Paris.

(1995), n 8. Lignes directrices établies à l'intention des organismes d'aide concernant les aspects régionaux et mondiaux de la mise en valeur et de la protection des milieux marins et littoraux, OCDE, Paris.

(1995), n 9 . Lignes directrices établies à l'intention des organismes d'aide pour une meilleure préservation et une utilisation durable des zones humides tropicales et subtropicales, OCDE, Paris.

OCDE (1995), L'aide des donneurs en faveur du développement des capacités dans le domaine de l'environnement, Paris.

OCDE (1995), Le développement participatif et la bonne gestion des affaires publiques, OCDE, Paris. 
OCDE (1995), Orientations du CAD concernant le rôle de la coopération pour le développement à l'appui de l'expansion du secteur privé, OCDE, Paris.

OCDE (1996), Assurer la cohérence des politiques : instruments et contraintes, Gestion publique, étude hors série $\mathrm{n}^{\circ} 12$, Paris.

OCDE (1996), Le rôle de la coopération pour le développement à l'aube du XXI siècle, OCDE, Paris.

OCDE (1998), Les conflits, la paix et la coopération pour le développement à l'aube du XXI siècle, OCDE, Paris.

OCDE (1998), Compendium du CAD de notions et approches relatives à l'égalité homme-femme, OCDE, Paris.

OCDE (1999), Comparaison des systèmes de gestion de la coopération au développement dans les pays de l'OCDE membres du CAD, OCDE, Paris.

OCDE (1999), Lignes directrices du CAD pour l'égalité homme-femme et le renforcement du pouvoir des femmes dans le cadre de la coopération pour le développement, OCDE, Paris.

OCDE (2000), Pratiques efficaces pour mener une évaluation conjointe associant plusieurs donneurs, OCDE, Paris.

OCDE (2001), Evaluation Feedback for Effective Learning and Accountability, OCDE, Paris.

OCDE (2001), «Lignes directrices du CAD » - Prévenir les conflits violents : quels moyens d'action ?, OCDE, Paris.

OCDE (2001), « Lignes directrices du CAD » - La réduction de la pauvreté, OCDE, Paris.

OCDE (2001), «Lignes directrices du CAD »- Stratégies de développement durable, OCDE, Paris.

OCDE (2001), «Lignes directrices du CAD »-Renforcer les capacités commerciales au service du développement, OCDE, Paris.

OCDE (2001), Articles sur la prévention des conflits et la coopération pour le développement, Les Dossiers du CAD, volume 2, $\mathrm{n}^{\circ}$ 3, OCDE, Paris.

OCDE (2002), Gender Equality in Sector Wide Approaches, OCDE, Paris.

OCDE (2003), « Lignes directrices et ouvrages de référence du CAD »-Harmoniser l'aide pour renforcer son efficacité, OCDE, Paris.

OCDE (2003), L'efficacité de l'aide et la sélectivité : intégrer de multiples objectifs dans les décisions d'affectation de l'aide, OCDE, Paris.

OCDE (2003), L'opinion publique contre la pauvreté, OCDE, Paris.

OCDE/OMS (2003), Lignes directrices et ouvrages de référence du CAD - Pauvreté et santé, OCDE, Paris.

OCDE (2004), Coopération pour le développement - Rapport 2003, OCDE, Paris.

OCDE (n.d.), Gender Equality Tip Sheets, OCDE, Paris.

Princeton Survey Research Associates (2003), Global Poll: Multinational Survey of Opinion Leaders 2002. Washington DC.

Royal Danish Ministry of Foreign Affairs (2003), A World of Difference, Copenhague.

The Simons Report (1997), One Clear Objective: Poverty Reduction through Sustainable Development: Report of the Committee of Review, Canberra.

Nations unies (2003), Application de la Déclaration du millénaire adoptée par l'Organisation des Nations unies : Rapport du Secrétaire général. New York. Ref. A/58/323.

Division de la Population des Nations unies (2003), Perspectives démographiques mondiales, Révision 2002, New York.

Banque mondiale (2002), World Bank Group Work in Low-Income Countries Under Stress : A Task Force Report, Washington DC.

Banque mondiale/Fonds monétaire international (2002), A Review of the Poverty Reduction Strategy Paper Approach, Banque mondiale, Washington DC.

Banque mondiale (2003), Rapport 2004 sur le développement dans le monde: Accès aux services essentiels pour les plus pauvres, Banque mondiale, Washington DC. 
LES ÉDITIONS DE L'OCDE, 2, rue André-Pascal, 75775 PARIS CEDEX 16 IMPRIMÉ EN FRANCE

(43 2005122 P) ISBN 92-64-00762-8 - nº 538882005 


\section{Lignes directrices et ouvrages de référence du CAD}

\section{La gestion de l'aide PRATIQUES DES PAYS MEMBRES DU GAD}

Les membres du Comité d'aide au développement (CAD) de l'OCDE consacrent chaque année près de 70 milliards USD à l'aide extérieure (la coopération pour le développement). Comment gérer au mieux cette aide ? Tel est le thème de la présente étude qui analyse les structures et les pratiques organisationnelles de 22 des principaux pays donneurs du monde. Plus que jamais dans le passé, l'attention se porte à présent sur les moyens d'améliorer l'efficacité de l'aide au moment même où le nombre de pays donneurs ne cesse de croître. Ces deux facteurs se conjuguent pour justifier que l'on veille tout particulièrement à disposer d'informations à jour sur la manière dont les donneurs possédant de nombreuses années d'expérience gèrent leurs programmes d'aide, et à dégager de bonnes pratiques énoncées sous une forme aisément accessible.

Intitulé la gestion de l'aide : pratiques des pays membres du CAD, le présent ouvrage, s'inspirant directement des enseignements de l'expérience acquise par les pays membres, aborde des thèmes aussi variés que le cadre juridique dans lequel s'inscrit la coopération pour le développement, la manière dont les donneurs organisent leurs activités dans les pays en développement partenaires, les mérites respectifs de la gestion centralisée et de la gestion décentralisée, les relations avec les organisations non gouvernementales, ainsi que la gestion de l'égalité homme-femme, la viabilité écologique et l'action humanitaire. II décrit la réorganisation et la réforme des organismes d'aide engagées pour améliorer leur capacité de concourir à la réalisation des objectifs du millénaire pour le développement et de relever le niveau et la qualité de vie de la moitié des habitants de la planète.

Cet ouvrage s'adresse aux responsables d'organismes d'aide, aux enseignants et aux étudiants travaillant sur les aspects théoriques et pratiques de la coopération pour le développement, ainsi qu'au personnel des organisations internationales et multilatérales, des organisations de la société civile et du secteur privé qui ont à cœur de mieux comprendre le fonctionnement des organismes d'aide au développement.

Le texte complet de cet ouvrage est disponible en ligne à l'adresse suivante :

http://new.sourceocde.org/developpement/926400761X

Les utilisateurs ayant accès à tous les ouvrages en ligne de l'OCDE peuvent également y accéder via : http://new.sourceocde.org/926400761X

SourceOCDE est une bibliothèque en ligne qui a reçu plusieurs récompenses. Elle contient les livres, périodiques et bases de données statistiques de l'OCDE. Pour plus d'informations sur ce service ou pour obtenir un accès temporaire gratuit, veuillez contacter votre bibliothécaire ou SourceOECD@oecd.org.

www.oecd.org

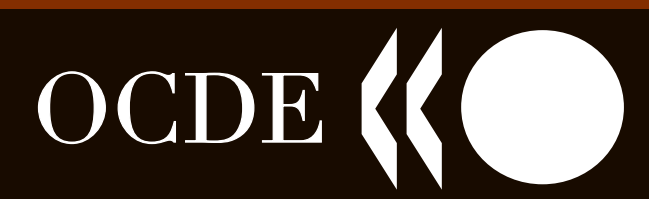

ÉDITIONSOCDE

ISBN 92-64-00762-8
$432005122 \mathrm{P}$ 